\title{
DETERMINING TIMESCALES OF NATURAL CARBONATION OF PERIDOTITE IN THE SAMAIL OPHIOLITE, SULTANATE OF OMAN
}

\author{
By \\ Evelyn Martinique Mervine
}

B.A. Earth Science and Arabic Language \& Literature, Dartmouth College, 2006

Submitted in partial fulfillment of the requirements for the degree of

\author{
Doctor of Philosophy \\ at the \\ ARCHMSO \\ MASSACHUSETTS INSTITUTE OF TECHNOLOGY \\ and the \\ WOODS HOLE OCEANOGRAPHIC INSTITUTION
}

June 2012

(C) 2012 Evelyn Martinique Mervine

All rights reserved.

The author herby grants to MIT and WHOI permission to reproduce and to distribute publicly paper and electronic copies of this thesis document in whole or in any part in any medium now known or hereafter created.

Signature of Author

Joint Program in Mátine Geology and Ğeophysics Massachusetts Institute of Technology and Woods Hole Oceanographic Institution

Certified by May 4th, 2012

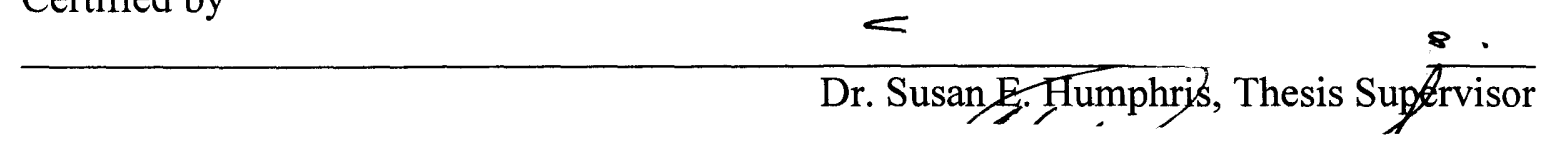

Dr. Kenneth W. W. Sims, Thesis Supervisor Accepted by

Dr. Robert L. Evans

Chair, Joint Committee for Marine Geology and Geophysics Massachusetts Institute of Technology/ Woods Hole Oceanographic Institution 



\title{
Determining Timescales of Natural Carbonation of Peridotite in the Samail Ophiolite, Sultanate of Oman
}

By Evelyn Martinique Mervine

Submitted to the Joint Committee for Marine Geology and Geophysics on May 4th, 2012 in partial fulfillment of the requirements for the degree of Doctor of Philosophy

\begin{abstract}
:
Determining timescales of the formation and preservation of carbonate alteration products in mantle peridotite is important in order to better understand the role of this potentially important sink in the global carbon cycle and also to evaluate the feasibility of using artificially-enhanced, in situ formation of carbonates in peridotite to mitigate the buildup of anthropogenic $\mathrm{CO}_{2}$ emissions in the atmosphere. Timescales of natural carbonation of peridotite were investigated in the mantle layer of the Samail Ophiolite, Sultanate of Oman. Rates of ongoing, low-temperature $\mathrm{CO}_{2}$ uptake were estimated through ${ }^{14} \mathrm{C}$ and ${ }^{230} \mathrm{Th}$ dating of carbonate alteration products. Approximately $1-3 \times 10^{6}$ $\mathrm{kg} \mathrm{CO}_{2} / \mathrm{yr}$ is sequestered in Ca-rich surface travertines and approximately $10^{7} \mathrm{~kg} \mathrm{CO}_{2} / \mathrm{yr}$ is sequestered in $\mathrm{Mg}$-rich carbonate veins. Rates of $\mathrm{CO}_{2}$ removal were estimated through calculation of maximum erosion rates from cosmogenic ${ }^{3} \mathrm{He}$ measurements in partiallyserpentinized peridotite bedrock associated with carbonate alteration products. Maximum erosion rates for serpentinized peridotite bedrock are $\sim 5$ to $180 \mathrm{~m} / \mathrm{Myr}$ (average: $\sim 40$ $\mathrm{m} / \mathrm{Myr}$ ), which removes at most $10^{5}-10^{6} \mathrm{~kg} \mathrm{CO}_{2} / \mathrm{yr}$ through erosion of $\mathrm{Mg}$-rich carbonate veins.
\end{abstract}




\section{Dedication}

I would like to dedicate my thesis to James "The Amazing" Randi, who taught me to think critically about the world around me and who reminds me that a $\mathrm{PhD}$ only means that I know a great amount about very little 


\section{Acknowledgments:}

First and foremost, I give my deepest thanks to my WHOI advisor Susan Humphris. Susan was an excellent mentor and role model, and she supported me through many difficult time periods and transitions during the course of my $\mathrm{PhD}$ journey. In the midst of an incredibly busy schedule, Susan always made time for me. Time and again, she went above and beyond the normal obligations of a $\mathrm{PhD}$ advisor. She even flew all the way to Muscat, Oman for three days to help me prepare for a conference presentation. Susan always pushed me to do my very best work, and she made sure that I had the support and resources that I needed in order to do my best work. I have no doubt that without Susan's mentorship I would not be here today, submitting my PhD thesis. Susan's support over the years has been invaluable.

I also owe sincere thanks to my co-advisor Ken Sims. When I decided to leave my first $\mathrm{PhD}$ project, Ken immediately offered to take me on as a student. When I asked if it would be possible for me to work in the Samail Ophiolite, Ken helped me develop a collaboration with Peter Kelemen and wrote grants to support the new research project. Even after Ken moved from WHOI to the University of Wyoming, he continued to support my work and even brought me out to Laramie for a summer. Ken flew back to WHOI numerous times to help me finish my $\mathrm{PhD}$, and he endured many long Skype calls of my questions.

I also owe thanks to my PhD committee members, particularly Peter Kelemen. Peter was excited and supportive when I expressed my desire to work in the Samail Ophiolite, and he helped me develop the research that now comprises my $\mathrm{PhD}$ thesis. Sam Bowring provided much sage advice about geochronology, graduate school, and life in general. Dan McCorkle helped me navigate my advisor transitions and also provided valuable scientific input and constructive criticism. Bill Jenkins was a source of expert

${ }^{14} \mathrm{C}$ advice and he also challenged me to think about the broader implications of my thesis research. Finally, I owe thanks to my committee chair Bernhard Peucker-Ehrenbrink, who ensured that my thesis defense was well-organized and went smoothly.

I would also like to thank my original advisor Fred Frey. Although I decided not to continue the Ninetyeast Ridge research project for my $\mathrm{PhD}$, Fred brought me to MIT and supported me for two years. During the time I worked with Fred, I learned a great amount about geochemistry and geochronology. I am grateful that Fred passed on to me a small fraction of his extensive knowledge. I also very much enjoyed participating in a two month expedition in 2007 to the Ninetyeast Ridge. I met good friends and colleagues - and also my now-husband - on the expedition, and it was an experience of a lifetime.

In addition to my advisors and committee members, I have many scientific collaborators and supporters to thank. Ann McNichol, Mark Roberts, Al Gagnon, and the rest of the NOSAMS staff are thanked for welcoming me into the laboratory and teaching me about ${ }^{14} \mathrm{C}$ dating. I am grateful to Chris Weidman for allowing me to subsample my carbonates using his microsampler. Margaret Sulanowska helped with sample preparation and XRD analysis. Lora Wingate provided the stable isotope analyses in a quick and professional manner. Chris Waters and Lynne Elkins, who were my fellow students under 
Ken, taught me about uranium-series geochemistry, both in the lab and outside of the lab. Mark Kurz and Josh Curtice provided mentorship and laboratory assistance for the cosmogenic ${ }^{3} \mathrm{He}$ measurements. I also owe thanks to Steve Richardson, who graciously provided me with peridotite thin sections free of charge, Henry Dick, who provided me with important information about the petrology and alteration of peridotites, and Frieder Klein, who shared his knowledge about carbonation of peridotite. Vera Pavel and Claire Pontbriand provided assistance with MATLAB programming. I also owe special thanks to Jurek Blusztajn, who was a constant source of support in the geochemistry and mass spectrometry labs. I especially appreciate Jurek's cheerful nature and infectious optimism, which were helpful when I was analyzing my most challenging samples.

I also owe thanks to many people for support, scientific discussions, and adventures in the field in Oman. Peter Kelemen's field experience was highly valuable. I also wish to give thanks to Ali Al-Rajhi, Ministry of Commerce and Industry, Sultanate of Oman, and Sobhi Nasir, Sultan Qaboos University, for logistical support during field seasons. Ken Sims, Jürg Matter, Everett Shock, Lisa Streit, Amelia Paukert, and other members of the field team are also thanked. I especially want to thank my husband Ernest (Jackie) Gauntlett, who took three and a half weeks of vacation time to be my field assistant during the 2010 season.

I am very grateful to have been part of the MIT/WHOI Joint Program, which provided me with wonderful opportunities and support. I wish to thank Jim Yoder, Meg Tivey, Julia Westwater, Marsha Gomes, Tricia Gebbie, and other members of the Academic Programs Office for their support. I particularly enjoyed the Geodynamics Program, which gave me the opportunity to participate in geology field trips to Costa Rica, Barbados, Switzerland, and Italy. These trips provided a welcome break from labwork and helped remind me why I love geology so much.

My friends and fellow classmates in the Joint Program were also incredibly supportive. We helped each other survive graduate school-and we often had good fun along the way. In particular, I wish to thank Vera Pavel, Jimmy Elsenbeck, Claire \& Cliff Pontbriand, Arthur Olive, Michael Holcomb, Emily \& Casey Saenger, Alison Criscitiello, Lynne Elkins, Chris Waters, Matt Jackson, Maya Bhatia, Andrea Burke, Helen Feng, Li Ling Hamady, Elise Olson, Meredith White, Dan Ohnemus, Karin Lemkau, Camilo Ponton, Min Xu, Fern Gibbons, Holly Owens, Christy Till, Jay Barr, and Mike Krawczynski. I also wish to thank my friends outside of graduate school, particularly my friends in South Africa. John, Joy, Davor, Cathy, Nia, Patrick, Kaylee, Nick, Greg, Andrea, Vere, Marius, and Helen - thank you for making me feel at home in South Africa and for sometimes fetching books for me from the University of Cape Town library.

Since November 2010 I have written a geology blog and been involved with the geoblogosphere, an online community of amateur and professional geologists. I wish to thank the American Geophysical Union for hosting my blog and the entire geoblogosphere for support, advice, and fun discussions. In particular, I'd like to thank geobloggers Dana Hunter, Callan Bentley, Jessica Ball, Anne Jefferson, Chris Rowan, Erik Klemetti, Brian Romans, Lockwood DeWitt, Ron Schott, and Ryan Brown. 
Finally, I owe great thanks to my family, particularly my parents Mark and Barbara and my sister Aynsley. My parents ensured that I received an excellent education starting from a young age, and they were an incredible source of strength during my $\mathrm{PhD}$. Randi, my adopted grandfather, and his partner José have also been a strong source of encouragement and support. Words cannot express my gratitude to my husband Jackie, who supported me during the best and the worst of graduate school. Long distance relationships are difficult, particularly when they stretch from Cape Cod to Cape Town. Jackie was incredibly supportive of my $\mathrm{PhD}$ - in person or, more often, over Skypeduring five years of long distance. He patiently endured my moments of despair and refused to let me quit, even when I was convinced that there was no way I could continue. Jackie also supported my $\mathrm{PhD}$ by cooking me many delicious dinners, figuring out how to move my books (and cats) to South Africa during my last year of graduate school, and chatting with me about geology during hikes and over countless cups of rooibos tea.

My $\mathrm{PhD}$ thesis research was funded by the National Science Foundation grant EAR-1049281, the Deep Ocean Exploration Institute at WHOI, the Academic Programs Office at WHOI, and the Mellon Foundation. 
Table of Contents:

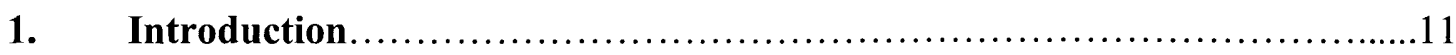

1. Natural Carbonation of Peridotite..........................................15

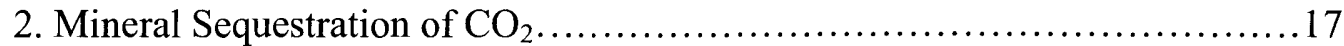

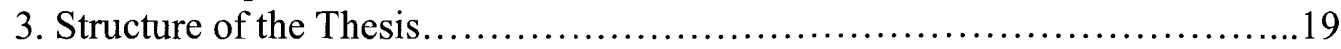

2. ${ }^{14} \mathrm{C}$ Dating of Carbonate Alteration Products of Peridotite in the Samail Ophiolite, Sultanate of Oman ......................................... 28

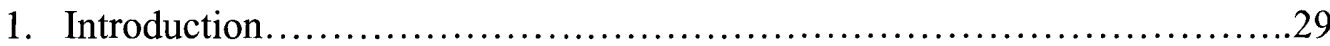

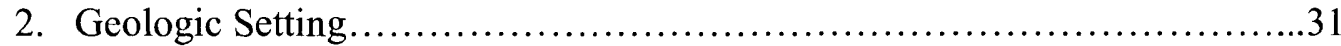

2.1 Geology of the Samail Ophiolite.................................. 31

2.2 Carbonate Alteration Products Formed in the

Samail Ophiolite................................................. 32

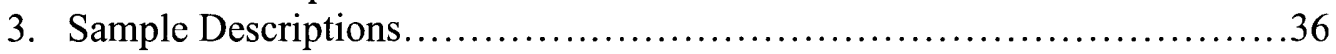

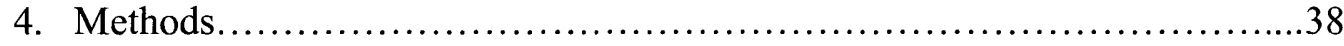

5. Results.............................................................

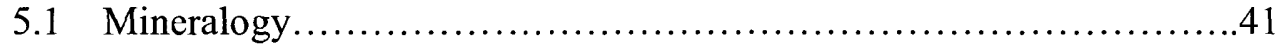

5.1 .1 Travertines..............................................42

5.1.2 Mg-Rich Carbonate Veins..................................43

5.2 Distribution of ${ }^{14} \mathrm{C}$ Ages.......................................... 43

5.2 .1 Travertines.................................................. 44

5.2.2 Mg-Rich Carbonate Veins..................................49

5.3 Stable Isotope Analyses......................................... 50

6. Discussion........................................................ 51

6.1 Temporal and Spatial Trends in Carbonate Formation................51

6.1.1 Surface Travertine Deposition and Preservation..................51

6.1.2 Mg-Rich Carbonate Veins...................................53

6.1 .3 Isotopic Variability......................................56

6.2 Carbonate Formation Temperatures.............................57

6.3 Estimating Natural Rates of Carbonate Formation in the Peridotite Layer of the Samail Ophiolite...........................................59

6.3.1 Travertine Deposition Rates.................................59

6.3.2 Natural $\mathrm{CO}_{2}$ Sequestration in $\mathrm{Mg}$-Rich Carbonate Veins..........61

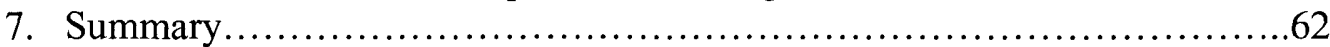

8. Acknowledgments.................................................. 64

3. ${ }^{230}$ Th Dating of Carbonate Alteration Products of Peridotite in the Samail Ophiolite, Sultanate of Oman: Applications and Limitations..............111

1. Introduction......................................................... 112

2. Geologic Setting..................................................... 113

3. Sample Descriptions................................................... 14

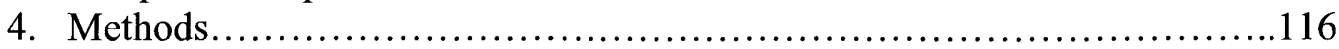




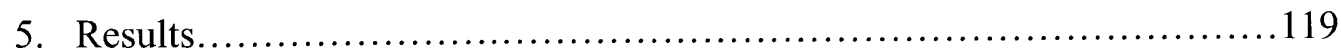

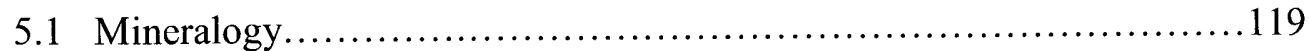

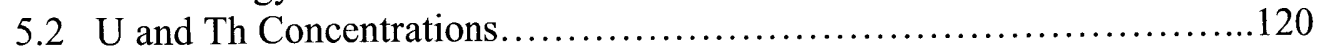

$5.2 \mathrm{U}$ and Th Isotopes.................................................

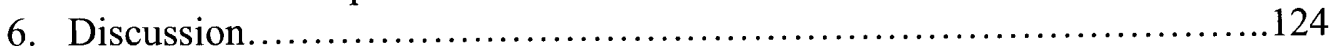

6.1 Systematics of $U$ and Th in Samail Carbonates, Peridotites, and

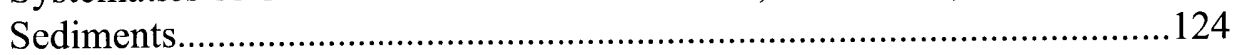

6.1.1 Systematics of $\mathrm{U}$ and $\mathrm{Th}$ Concentrations.........................124

6.1.2 Systematics of $U$ and Th Isotopes..............................127

6.2 Approaches and Limitations for Determining ${ }^{230} \mathrm{Th}$ Ages for Samail

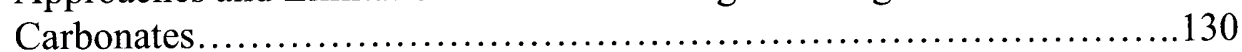

6.2.1 ${ }^{230}$ Th Dating of Terrestrial Carbonates................................ 130

6.2.2 ${ }^{230}$ Th Dating of Travertines................................... 136

6.2.3 ${ }^{230 \mathrm{Th}}$ Dating of Mg-Rich Carbonate Veins.........................138

6.3 Implications for Natural Rates of Carbonate Formation in the Peridotite Layer of the Samail Ophiolite........................................ 140

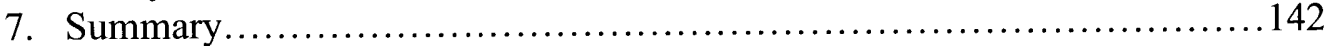

8. Acknowledgments.................................................... 143

\section{4. ${ }^{3}$ He Exposure Ages and Erosion Rates of Serpentinized Peridotite Bedrock,} Samail Ophiolite, Sultanate of Oman .....................................175

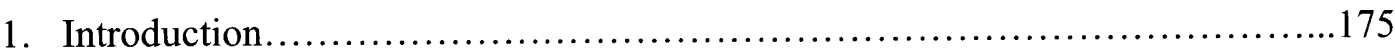

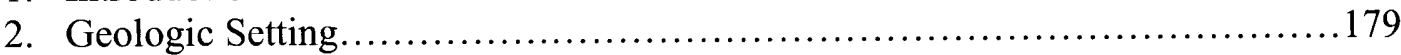

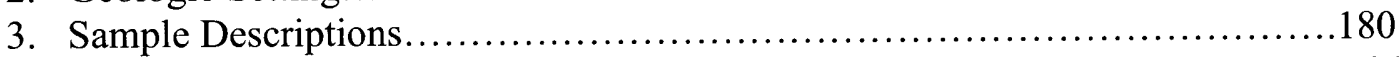

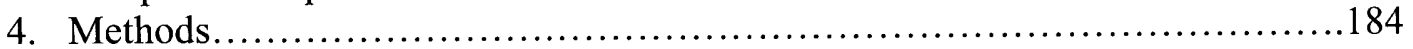

4.1 Sample Preparation................................................... 184

4.2 Helium Measurements............................................... 184

5. Results and Discussion.................................................... 185

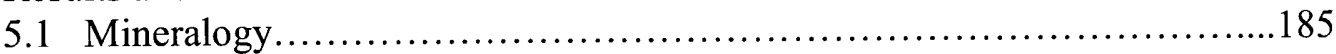

5.2 Helium Measurements................................................ 186

$5.2 .1{ }^{4} \mathrm{He}$ Concentrations........................................... 186

5.2 .2 He Isotopes.................................................... 187

5.3 Radiogenic Production of ${ }^{4} \mathrm{He}$ from $\mathrm{U}$ and $\mathrm{Th}$ Decay......................190

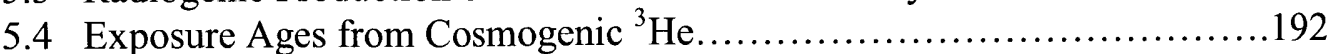

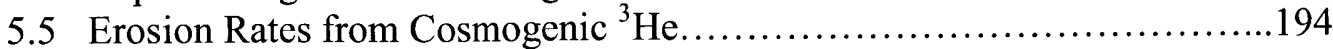

5.6 Implications for Erosion of the Peridotite Layer of the Samail Ophiolite...195

5.6.1 Ultramafic Erosion Rates...............................................195

5.6.2 Implications for Natural $\mathrm{CO}_{2}$ Sequestration in the Samail

Ophiolite........................................................... 196

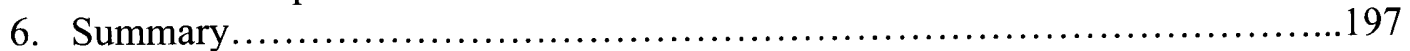

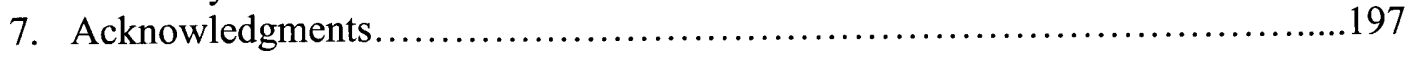


5. Synthesis and Future Directions

.225

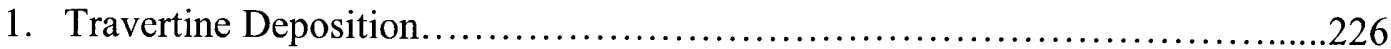

2. Formation of Mg-Rich Carbonate Veins..................................229

3. Beyond the Samail Ophiolite.........................................231

Appendix A: Carbonate Dissolution Experiments for U and Th Analyses..............239

Appendix B: Additional Sensitivity Analysis Figures for Initial ${ }^{230}$ Th Corrections of

${ }^{230} \mathrm{Th}$ Ages for Samail Carbonates................................258 


\section{Chapter 1: Introduction}

Alteration of ultramafic rocks such as peridotite is a poorly constrained category of silicate weathering. Although silicate weathering is well-established as a significant sink for carbon (e.g. Ebelmen, 1845; Rubey, 1951; Walker et al., 1981; Raymo and Ruddiman, 1992; Berner and Berner, 1997; Gaillard et et al., 1999; Berner and Kothavala, 2001; Dessert et al., 2003; Gislason et al., 2006; Kashiwagi et al., 2008; Gislason and Oelkers, 2011), one of the greatest sources of uncertainty in the carbon cycle is the amount of carbon stored in silicate rocks, including mafic and ultramafic lithologies (e.g. Holser et al., 1988; Sundquist, 1993; Gislason and Oelkers, 2011). While carbonation of mafic and ultramafic rocks is commonly observed, the amount of carbon stored globally in these carbonate alteration products has not been adequately quantified. Constraining rates of mafic and ultramafic weathering and carbonation is essential in order to fully understand and balance the global carbon cycle and also to evaluate the viability of using artificially-enhanced weathering of mafic and ultramafic rocks to mitigate the buildup of anthropogenic $\mathrm{CO}_{2}$ input to the atmosphere (e.g. Seiftritz, 1990; Lackner et al., 1995; Lackner, 2002; Kelemen and Matter, 2008; Matter and Kelemen, 2009; Gislason et al., 2010; Kelemen et al., 2011).

A few studies have attempted to quantify the amount of carbon stored in mafic basalts. Based on chemical compositions of small rivers draining basalts, Dessert et al. 
(2003) estimated that chemical weathering of basalts consumes $1.8 \times 10^{11} \mathrm{~kg} \mathrm{CO}_{2} / \mathrm{yr}^{1}$. This result is significant because although basalt represents only $\sim 5 \%$ of the continental surface, basalt weathering constitutes $\sim 30-35 \%$ of the $\sim 5.15 \times 10^{11} \mathrm{~kg} \mathrm{CO}_{2} / \mathrm{yr}$ consumed by continental silicate weathering (Gaillardet et al., 1999; Dessert et al., 2003). Two studies have estimated $\mathrm{CO}_{2}$ uptake rates in seafloor basalt (Staudigel et al., 1989; Alt and Teagle, 1999). Study of carbonate contents of basalts from three drill sites (Holes 417A, 417D, and 418A) near Bermuda and four drill sites (Sites 504, 801, 843, and 896) in the Pacific has led to the estimate that $\sim 1.5 \times 10^{11} \mathrm{~kg} \mathrm{CO}_{2} / \mathrm{yr}$ is stored through carbonate alteration of seafloor basalt (Staudigel et al., 1989; Alt and Teagle, 1999). Since ocean ridge volcanism releases $\sim 4.4-8.4 \times 10^{10} \mathrm{~kg} \mathrm{CO}_{2} / \mathrm{yr}$ (Gerlach, 1989), a net sink of $\sim 0.66$ $1.1 \times 10^{11} \mathrm{~kg} \mathrm{CO}_{2} / \mathrm{yr}$ is stored through carbonation of seafloor basalt (Staudigel et al., 1989; Alt and Teagle, 1999).

Although not as abundant as mafic exposures, there are considerable exposures of ultramafic rocks at Earth's surface. In addition to being subaerially exposed at ophiolites, large volumes of peridotite are exposed on the global mid-ocean ridge system along ridge discontinuities, rift valley walls, and detachment faults. In fact, Escartin et al. (2008) estimate that detachment faulting occurs along $50 \%$ of the Mid-Atlantic Ridge between $12-35^{\circ} \mathrm{N}$, implying that extensive seafloor exposures of peridotite are common. Furthermore, ultramafic rocks are expected to weather and erode more quickly than other types of silicate rocks due to their chemical composition and texture (e.g. Luce et al.,

\footnotetext{
${ }^{1}$ Units of $\mathrm{kg}$ of $\mathrm{CO}_{2}$ are used throughout this thesis. However, $\mathrm{CO}_{2}$ emissions are commonly reported in metric gigatons $(\mathrm{Gt})$ while carbon cycle reservoirs and fluxes are commonly reported in moles or $\mathrm{Pg}$ (pentagrams, $1 \mathrm{Pg}=10^{15} \mathrm{~g}=10^{9} \mathrm{t}$ ) of C. Note that $1 \mathrm{Gt} \mathrm{CO}_{2}=10^{9} \mathrm{~kg} \mathrm{CO}_{2}=2.7 \times 10^{8} \mathrm{~kg} \mathrm{C}=6.2 \times 10^{9} \mathrm{~mol}$ C.
} 
1972; Hänchen et al., 2006; Matter and Kelemen, 2009; Kelemen et al., 2011). In addition, both subaerial and seafloor ultramafic rocks are known to take up significant $\mathrm{CO}_{2}$ in secondary alteration minerals (e.g. Barnes et al., 1967, 1978; Barnes and O'Neil, 1969; Trommsdorff and Evans, 1977; Trommsdorff et al., 1980; Ferry, 1995; Surour and Arafa, 1997; Kelley et al., 2001; Früh-Green et al., 2003; Ludwig et al., 2006; 2011; Wilson et al., 2006, 2009a, 2009b; Kelemen and Matter, 2008; Matter and Kelemen, 2009; Kelemen et al., 2011). Therefore, even though ultramafic rocks are less abundant than other types of silicate rocks on Earth's surface, the influence of ultramafic rocks on the carbon cycle may nonetheless be important.

Despite the potential importance, the role of ultramafic rocks in the carbon cycle has not been extensively studied or quantified. Studies of ultramafic rocks and $\mathrm{CO}_{2}$ have largely focused on $\mathrm{CO}_{2}$ budgets at subduction zones (e.g. Kerrick and Connolly, 1998; Früh-Green et al., 2001; Gorman et al., 2006; Spandler et al., 2008) or in the deep mantle (e.g. Brenker et al., 2007; Dasgupta and Hirschmann, 2010) while only a few studies have investigated rates of $\mathrm{CO}_{2}$ uptake during weathering of ultramafic rocks (e.g. Wilson et al., 2006, 2009a, 2009b; Kelemen and Matter, 2008). Wilson et al. (2006, 2009a, $2009 \mathrm{~b}$ ) investigated active carbonation of kimberlite diamond mine tailings in northern Canada and demonstrated that these mine tailings sequester on the order of $10^{6} \mathrm{~kg} \mathrm{CO}_{2} / \mathrm{yr}$ for each mine. The amount of $\mathrm{CO}_{2}$ sequestered annually in kimberlite mine tailings is small relative to $\sim 35 \times 10^{12} \mathrm{~kg}$ of anthropogenic $\mathrm{CO}_{2}$ emitted in 2010 (Friedlingstein et al., 2010). Nonetheless, the annual $\mathrm{CO}_{2}$ sequestration capacity of a large ultramafic mine can exceed its annual $\mathrm{CO}_{2}$ emissions (Wilson et al., 2009a). However, alteration rates in 
ultramafic mine tailings are likely accelerated by the dramatic increase in surface area that occurs during milling of ore and hence are different from natural carbonation rates of ultramafic bedrock. A previous study of carbonate formation in the peridotite layer of the Samail Ophiolite in the Sultanate of Oman by Kelemen and Matter (2008) estimated that there is $\sim 10^{12} \mathrm{~kg} \mathrm{CO}_{2}$ stored in carbonate alteration products of peridotite and that the peridotite layer consumes $\sim 4 \times 10^{7} \mathrm{~kg} \mathrm{CO}_{2} / \mathrm{yr}$. While Samail is a large ophiolite, it contains only a fraction of the ultramafic rock exposed globally.

Similarly, only a few studies have investigated erosion of ultramafic rocks (e.g. Moretti and Turcotte, 1985; Freyssinet and Farah, 2000; Beauvais et al., 2007; Hanson et al., 2009), and none have employed measurement of cosmogenic nuclides, which can be used to place quantitative constraints on erosion rates. Ultramafic erosion rates are important because they place limits on rates of $\mathrm{CO}_{2}$ uptake and storage in ultramafic rocks, particularly via low-temperature carbonation believed to occur primarily in a nearsurface weathering zone (e.g. Kelemen and Matter, 2008; Kelemen et al., 2011). Ultramafic weathering rates are also important to consider when investigating the development of economic deposits of metals such as nickel, platinum, chromium, cobalt, and manganese in soils derived from ultramafic rocks (e.g. Trescases, 1973; Elias et al., 1981; Golightly, 1981, 2010; Summons et al., 1981; Nahon et al., 1982; Llorca and Monchoux, 1991; Cook and Fletcher, 1993, 1994; Fletcher et al., 1995; de Oliveira et al., 2001; Gleeson et al., 2003, 2004; Yongue-Fouateu et al., 2006; Dzemua and Gleeson, 2012) and are locally important in regions dominated by ultramafic bedrock (e.g. mantle peridotite, kimberlite, and komatiite). 
The goal of the research presented in this thesis is to provide better constraints on natural peridotite carbonation and erosion rates in the Samail Ophiolite, Sultanate of Oman. The Samail Ophiolite is one of the largest and best-exposed ophiolites in the world and hence is an ideal natural laboratory for the investigation of subaerial peridotite carbonation and erosion. Because the Samail Ophiolite is located in an arid (modern) climate, rates of carbonate formation and erosion in Samail peridotite likely represent slow end-members, and these rates may be much faster in more humid environments. In this thesis, timescales of peridotite carbonation have been constrained through ${ }^{14} \mathrm{C}$ dating and ${ }^{230} \mathrm{Th}^{2}{ }^{238} \mathrm{U}$ dating of Samail carbonates as well as through $\mathrm{U}$ and $\mathrm{Th}$ systematics of Samail carbonates and peridotites. Interpretation of the age information gleaned from the ${ }^{14} \mathrm{C}$ and $\mathrm{U}-\mathrm{Th}$ systems was supported by $\mathrm{C}$ and $\mathrm{O}$ isotopic analyses on carbonates and by mineralogical identifications using X-ray diffraction. Timescales of peridotite erosion were investigated through measurement of cosmogenic ${ }^{3} \mathrm{He}$ in partially serpentinized peridotite bedrock.

\section{Natural Carbonation of Peridotite:}

Mantle peridotite is composed primarily of the minerals olivine $\left[(\mathrm{Mg}, \mathrm{Fe})_{2} \mathrm{SiO}_{4}\right]$ and pyroxene $\left[(\mathrm{Mg}, \mathrm{Ca}, \mathrm{Fe})_{2} \mathrm{Si}_{2} \mathrm{O}_{6}\right]$, which are far from equilibrium with $\mathrm{H}_{2} \mathrm{O}$ and $\mathrm{CO}_{2}$ at Earth's surface and thus are easily altered to hydrous silicates (e.g. serpentine), Fe-oxides (e.g. magnetite), and carbonates (e.g. calcite, magnesite, dolomite). Very generalized reactions for this peridotite hydration and carbonation are (e.g. Lacker, 2002; Kelemen and Matter, 2008; Kelemen et al., 2011): 
Forsterite (Mg-olivine) hydration and carbonation:

$$
\begin{aligned}
& 4 \mathrm{Mg}_{2} \mathrm{SiO}_{4}+4 \mathrm{H}_{2} \mathrm{O}+2 \mathrm{CO}_{2}=2 \mathrm{Mg}_{3} \mathrm{Si}_{2} \mathrm{O}_{5}(\mathrm{OH})_{4}+2 \mathrm{MgCO}_{3} \\
& \text { forsterite serpentine magnesite }
\end{aligned}
$$

The above reaction often occurs through formation of intermediate brucite:

$$
\begin{aligned}
& 4 \mathrm{Mg}_{2} \mathrm{SiO}_{4}+6 \mathrm{H}_{2} \mathrm{O}=2 \mathrm{Mg}_{3} \mathrm{Si}_{2} \mathrm{O}_{5}(\mathrm{OH})_{4}+2 \mathrm{Mg}(\mathrm{OH})_{2} \\
& \text { forsterite serpentine brucite } \\
& 2 \mathrm{Mg}(\mathrm{OH})_{2}+2 \mathrm{CO}_{2}=2 \mathrm{MgCO}_{3}+2 \mathrm{H}_{2} \mathrm{O} \\
& \text { brucite magnesite }
\end{aligned}
$$

Varying proportions of $\mathrm{H}_{2} \mathrm{O}$ and $\mathrm{CO}_{2}$ lead to the formation of talc and quartz:

$$
\begin{aligned}
& 4 \mathrm{Mg}_{2} \mathrm{SiO}_{4}+\mathrm{H}_{2} \mathrm{O}+5 \mathrm{CO}_{2}=\mathrm{Mg}_{3} \mathrm{Si}_{4} \mathrm{O}_{10}(\mathrm{OH})_{2}+5 \mathrm{MgCO}_{3} \\
& \text { forsterite talc magnesite } \\
& 4 \mathrm{Mg}_{2} \mathrm{SiO}_{4}+8 \mathrm{CO}_{2}=4 \mathrm{SiO}_{2}+8 \mathrm{MgCO}_{3} \\
& \text { forsterite quartz magnesite }
\end{aligned}
$$

Fayalite (Fe-olivine) hydration:

$$
\begin{aligned}
& 6 \mathrm{Fe}^{2+} \mathrm{SiO}_{4}+7 \mathrm{H}_{2} \mathrm{O}=3 \mathrm{Fe}^{2+} \mathrm{Si}_{2} \mathrm{O}_{5}(\mathrm{OH})_{4}+\mathrm{Fe}_{2}{ }^{3+} \mathrm{Fe}^{2+} \mathrm{O}_{4}+\mathrm{H}_{2}(\mathrm{aq}) \\
& \text { fayalite } \mathrm{Fe} \text {-serpentine magnetite }
\end{aligned}
$$

Olivine and pyroxene hydration and carbonation:

$$
\begin{aligned}
& 4 \mathrm{Mg}_{2} \mathrm{SiO}_{4}+\mathrm{CaMgSi}_{2} \mathrm{O}_{6} \\
& \text { forsterite } \mathrm{CaMg} \text {-pyroxene }
\end{aligned} 6 \mathrm{H}_{2} \mathrm{O}+\mathrm{CO}_{2}=\underset{\text { serpentine }}{3 \mathrm{Mg}_{3} \mathrm{Si}_{2} \mathrm{O}_{5}(\mathrm{OH})_{4}}+\underset{\text { calcite }}{\mathrm{CaCO}_{3}}
$$

The above reaction often occurs in two stages:

$$
\left.\underset{\text { forsterite }}{4 \mathrm{Mg}_{2} \mathrm{SiO}_{4}}+\mathrm{CaMgSi}_{2} \mathrm{O}_{6}+7 \mathrm{H}_{2} \mathrm{O}=\underset{\text { serpentine }}{3 \mathrm{Mg}_{3} \mathrm{Si}_{2} \mathrm{O}_{5}(\mathrm{OH})_{4}}+\mathrm{Ca}^{2+(} \mathrm{aq}\right)+2 \mathrm{OH}^{-}(\mathrm{aq})
$$

occurs in the subsurface, and:

$$
\mathrm{Ca}^{2+}(\mathrm{aq})+2 \mathrm{OH}^{-}(\mathrm{aq})+\mathrm{CO}_{2}(\text { aq or gas })=\underset{\text { calcite }}{\mathrm{CaCO}_{3}+\mathrm{H}_{2} \mathrm{O}}
$$

occurs when fluids from the first stage react with $\mathrm{CO}_{2}$ to form travertine in the nearsurface. 
Alteration of olivine and pyroxene to hydrous and carbonate minerals occurs naturally at low temperatures in mafic and ultramafic rocks exposed to water near the atmosphere (i.e. there is a source of $\mathrm{H}_{2} \mathrm{O}$ and $\mathrm{CO}_{2}$ ). Thermodynamically, these reactions are exothermic and spontaneous (e.g. Klein and Garrido, 2011). However, they occur slowly in nature because of kinetic limitations, and the natural rates at which these reactions occur are not well constrained at present.

\section{Mineral Sequestration of $\mathrm{CO}_{2}$ :}

Considerable attention has focused recently on carbon capture and storage to mitigate anthropogenic input to atmospheric $\mathrm{CO}_{2}$ (e.g. Metz et al., 2005). Sequestration of $\mathrm{CO}_{2}$ in solid carbonate minerals has. a major advantage over alternative sequestration methods ${ }^{2}$ in that there is little risk of $\mathrm{CO}_{2}$ leaking back to the atmosphere (e.g. Seifritz, 1990; Lackner et al., 1995; Lackner, 2002; Oelkers et al., 2008). No monitoring is required to prevent escape of $\mathrm{CO}_{2}$, which will be released back to the atmosphere only as the carbonates weather over thousands to millions of years (e.g. Lackner, 2002; Oelkers et al., 2008). Most of the Earth's $\mathrm{CO}_{2}$ is already stored in solid minerals. There is $\sim 3.9 \mathrm{x}$ $10^{16} \mathrm{~kg} \mathrm{CO}_{2}$ presently stored in carbonate rocks (e.g. marble, limestone, chalk) in contrast to $\sim 4 \times 10^{13} \mathrm{~kg} \mathrm{CO}_{2}$ in the ocean and a mere $\sim 8 \times 10^{11} \mathrm{~kg} \mathrm{CO}_{2}$ in the atmosphere (Adams and Caldeira, 2008; Oelkers et al., 2008). Formation of carbonate minerals occurs

2 Other proposed options for $\mathrm{CO}_{2}$ sequestration include pumping $\mathrm{CO}_{2}$ underground to assist in enhanced petroleum recovery, storing $\mathrm{CO}_{2}$ in saline aquifers or deep sedimentary formations, and injecting $\mathrm{CO}_{2}$ into the deep ocean (Lackner, 2002; Adams and Caldeira, 2008; Benson and Cole, 2008; Oelkers and Cole, 2008; Oelkers et al., 2008). Storing $\mathrm{CO}_{2}$ underground as a liquid or gas is challenging because of the potential for slow or fast leaks and the difficulty of long-term monitoring (Lackner, 2002; Oelkers and Cole, 2008; Benson and Cole, 2008). $\mathrm{CO}_{2}$ injection into the deep ocean is at best a temporary solution as ocean circulation will return the $\mathrm{CO}_{2}$ to the ocean surface after $\sim 1000$ years (Adams and Caldeira, 2008). Further, addition of $\mathrm{CO}_{2}$ to the ocean leads to ocean acidification, a condition already plaguing the shallow ocean and having a detrimental influence on ocean biology (Orr et al., 2005; Adams and Caldeira, 2008). 
naturally with an estimated $1.4 \times 10^{8} \mathrm{~kg} \mathrm{CO}_{2}$ stored each year through continental silicate weathering, not including ultramafic lithologies (Gaillardet et et al., 1999; Oelkers et al., 2008).

The key to mineral sequestration of $\mathrm{CO}_{2}$ is to speed up natural rates of reaction so that more carbon is sequestered per year. There are two ways to approach this: ex situ mineralization and in situ mineralization. Ex situ mineralization involves the mining and transport of appropriate minerals (such as olivine or serpentine) to a carbonation reactor where the minerals are heated to induce reaction with a stream of concentrated $\mathrm{CO}_{2}$ (Oelkers et al., 2008; Lackner, 2002). Mining, transport, and heating all require energy, which makes ex situ mineralization costly and inefficient. In situ carbonation involves the direct injection of $\mathrm{CO}_{2}$ into porous or fractured bedrock. $\mathrm{CO}_{2}$ must still be captured and transported, but the financial and energy costs are much less than for ex situ mineralization. Additionally, because the carbonation reactions are exothermic, there is evidence that after some initial heat supply the reactions may become self-sustaining (e.g. Kelemen and Matter, 2008).

Ultramafic terrains are ideal for in situ carbonation of bedrock because they are primarily composed of olivine and pyroxene, which have dissolution rates that are significantly faster than those of other silicate minerals (e.g. Luce et al., 1972; Van Herk et al., 1989; Wogelius and Walther, 1991; Chen and Brantley, 2000; Pokrovski and Schott, 2000; Giammar et al., 2004; Hänchen et al., 2006; Prigiobbe et al., 2009). Ultramafic rocks likely have faster carbonation rates than mafic rocks, which have 
already been targeted for in situ $\mathrm{CO}_{2}$ sequestration ${ }^{3}$ (O'Connor et al., 2004; Chizmeshya et al, 2007; Matter and Kelemen, 2009; Kelemen et al., 2011).

\section{Structure of the Thesis:}

This thesis consists of five chapters. Chapter 2 contains the results of ${ }^{14} \mathrm{C}$ dating as well as $\mathrm{C}$ and $\mathrm{O}$ isotopic and mineralogical analyses of carbonate alteration products formed in the mantle peridotite layer of the Samail Ophiolite. Chapter 3 contains $U$ and Th concentration and isotopic analyses for Samail peridotites and their associated carbonate alteration products. Precise dating of Samail carbonates using the ${ }^{230} \mathrm{Th}$ dating technique was limited by low $U$ concentrations and relatively high $T h / U$ ratios. Nevertheless, comparison of ${ }^{230} \mathrm{Th}$ and ${ }^{14} \mathrm{C}$ ages and age limits, as well as the investigation of $U$ and $T h$ systematics during alteration of peridotite, provides important constraints on the timescales of natural carbonate formation in the peridotite layer of the Samail Ophiolite. Chapter 4 contains measurements of cosmogenic ${ }^{3} \mathrm{He}$ on partiallyserpentinized peridotite bedrock and determinations of minimum peridotite exposure ages and maximum peridotite erosion rates. Finally, Chapter 5 contains a synthesis of the three data chapters and suggests directions for future research.

\footnotetext{
${ }^{3}$ Terrestrial basaltic terrains have already been targeted for in situ mineral sequestration of $\mathrm{CO}_{2}$ with a pilot carbonation study called CarbFix in Iceland (Oelkers et al., 2008; Gislason et al., 2010). An organization called the Big Sky Carbon Sequestration Project is investigating the potential for carbonation of basalts in the northwestern US (http://www.bigskyco2.org).
} 


\section{References:}

Adams, E. and Caldeira, K. 2008. Ocean storage of $\mathrm{CO}_{2}$. Elements, Vol. 4: 319-324.

Akbulut, M., Piskin, O., and Karayigit, A.I. 2006. The genesis of the carbonatized and silicified ultramafics known as listwanites: A case study from the Mihaliccik region (Eskisehir), NW Turkey. Geological Journal, Vol. 41: 557-580.

Alt, J. and Teagle, D. 1999. The uptake of carbon during alteration of ocean crust. Geochimica et Cosmochimica Acta, Vol. 63, No. 10: 1527-1535.

Barnes, I., LaMarche, V.C., and Himmelberg, G. 1967. Geochemical evidence of present day serpentinization. Science, Vol. 156: 830-832.

Barnes, I. and O'Neil, J.R. 1969. The relationship between fluids in some fresh Alpine type ultramafics and possible modern serpentinization, Western United States. Geological Society of America Bulletin, Vol. 80: 1947-1960.

Barnes, I., O’Neil, J.R., and Trescases, J.J. 1978. Present-day serpentinization in New Caledonia, Oman, and Yugoslavia. Geochimica et Cosmochimia Acta, Vol. 42: 144-145.

Beauvais, A., Parisot, J.-C., and Savin, C. 2007. Ultramafic rock weathering and slope erosion processes in a South West Pacific tropical environment. Geomorphology, Vol. 83: 1-13.

Benson, S. and Cole, D. 2008. $\mathrm{CO}_{2}$ sequestration in deep sedimentary formations. Elements, Vol. 4: 325-331.

Berner, R. and Berner, E. 1997. Silicate weathering and climate. In: Ruddiman, W. (ed.) Tectonic Uplift and Climate Change. New York: Plenum Press, 353-365.

Berner, R. and Kothavala, Z. 2001. GEOCARB III: A revised model of atmospheric $\mathrm{CO}_{2}$ over Phanerozoic time. American Journal of Science, Vol. 301, No. 2: 182-204.

Brenker, F.E., Vollmer, C., Vincze, L., Vekemans, B., Szymanksi, A., Jassens, K., Szaloki, I., Nasdala, L., Joswig, W., and Kaminsky, F. 2007. Carbonates from the lower mantle part of transition zone or even the lower mantle. Earth and Planetary Science Letters, Vol. 260: 1-9.

Chen, Y. and Brantley, S.L. 2000. Dissolution of forsteritic olivine at $65^{\circ} \mathrm{C}$ and $2<\mathrm{pH} 5$. Chemical Geology, Vol. 165: 267-281. 
Chizmeshya, A.V.G., McKelvy, M.J., Squires, K., Carpenter, R.W., and Bérat, H. 2007. A novel approach to mineral carbonation: enhancing carbonation while avoiding mineral pretreatment costs. U.S. Department of Energy Final Report 924162, Arizona State University, Tempe, Arizona.

Cook, S.J. and Fletcher, W.K. 1993. Distribution and behaviour of platinum in soil, sediments, and waters of the Tulameen ultramafic complex, southern British Columbia, Canada. Journal of Geochemical Exploration, Vol. 46: 279-308.

Cook, S.J. and Fletcher, W.K. 1994. Platinum distribution in soil profiles of the Tulameen ultramafic complex, southern British Columbia. Journal of Geochemical Exploration, Vol. 51: 161-191.

Dasgupta, R. and Hirschmann, M. M. 2010. The deep carbon cycle and melting in Earth's interior. Earth and Planetary Science Letters, Vol. 298: 1-13.

de Oliveira, S.M.B., de Moya Partiti, C. S., and Enzweiler, J. 2001. Ochreous laterite: a nickel ore from Punta Gorda, Cuba. Journal of South American Earth Sciences, Vol. 14: 307-317.

Dessert, C., Dupré, B., Gaillardet, J., François, L.M., and Allègre, C. 2002. Basalt weathering laws and the impact of basalt weathering on the global carbon cycle. Chemical Geology, Vol. 202: 257-273.

Dzemua, G. L., and Gleeson, S.A. 2012. Petrography, mineralogy, and geochemistry of the Nkamouna serpentinite: Implications for the formation of the cobalt manganese laterite deposit, southeastern Cameroon. Economic Geology, Vol. 107: $25-41$.

Ebelmen, J. J. 1845. Sur les produits de la décomposition des especes minérals de la famille des silicates. Annales des Mines, Vol. 7: 3-66.

Escartin, J., Smith, D., Cann, J., Schouten, H., Langmuir, C., and Escrig, S. 2008. Central role of detachment faults in accretion of slow-spreading oceanic lithosphere. Nature, Vol. 455: 790-794.

Elias, M., Donaldson, M.J., and Giorgetta, N. 1981. Geology, mineralogy, and chemistry of lateritic nickel-cobalt deposits near Kalgoorlie, Western Australia. Economic Geology, Vol. 76: 1775-1783.

Ferry, J.M. 1995. Fluid-flow during contact-metamorphism of ophicarbonate rocks in the Bergell-Aureole, Val-Malenco, Italian Alps. Journal of Petrology, Vol. 36: 1039 1053. 
Fletcher, W.K., Cook, S.J., Hall, G.E.M., Scagel, R.K., and Dunn, C.E. 1995. Enrichment of platinum and associated elements in organic seepage soils of the Tulameen ultramafic complex, southern British Columbia. Journal of Geochemical Exploration, Vol. 54: 39-47.

Freyssinet, P. and Farah, A. S. Geochemical mass balance and weathering rates of ultramafic schists in Amazonia. Chemical Geology, Vol. 170: 133-151.

Früh-Green, G.L., Scambelluri, M., and Vallis, F. 2001. O-H isotope ratios of high pressure ultramafic rocks: implications for fluid sources and mobility in the subducted hydrous mantle. Contributions to Mineralogy and Petrology, Vol. 141: 145-159.

Früh-Green, G.L., Kelley, D.S., Bernasconi, S.M., Karson, J.A., Ludwig, K.A., Butterfield, D.A., Boschi, C., and Proskurowski, G. 2003. 30,000 years of hydrothermal activity at the Lost City vent field. Science, Vol. 301: 495-498.

Gaillardet, J., Dupré, B., Louvat, P., and Allègre, C. 1999. Global silicate weathering and $\mathrm{CO}_{2}$ consumption rates deduced from the chemistry of the large rivers. Chemical Geology, Vol. 159: 3-30.

Gerlach, T.M. 1989. Degassing of carbon dioxide from basaltic magma at spreading centers II. Mid-ocean ridge basalts. Journal of Volcanology and Geothermal Research, Vol. 39: 221-232.

Giammar, D.E., Bruant, R.G., and Peters, C.A. 2005. Forsterite dissolution and magnesite precipitation at conditions relevant for deep saline aquifer storage and sequestration of carbon dixide. Chemical Geology, Vol. 217: 257-276.

Gislason, S.R., Oelkers, E.H., and Snorrason, A. 2006. Role of river-suspended material in the global carbon cycle. Geology, Vol. 34, No. 1: 49-52.

Gislason, S.R., Wolff-Boenisch, D., Stefansson, A., Oelkers, E.H., Gunnlaugsson, E., Sigurdardottir, H., Sigfusson, B., Broecker, W.S., Matter, J.M., Stute, M., Axelsson, G.,and Fridriksson, T. 2010. Mineral sequestration of carbon dioxide in basalt: A pre-injection overview of the CarbFix project. International Journal of Greenhouse Gas Control, Vol. 4: 537-545.

Gislason, S.R. and Oelkers, E. H. 2011. Silicate rock weathering and the global carbon cycle. In:Harmon, R.S. and Parker, A. (eds.) Frontiers in Geochemistry: Contribution of Geochemistry to the Study of the Earth. Oxford: Blackwell Publishing Ltd., 84-103. 
Gleeson, S.A., Butt, C.R.M., and Elias, M. 2003. Nickel laterites: A review. Society of Economic Geologists Newsletter, Vol. 54, No. 1: 12-18.

Gleeson, S.A., Herrington, R.J., Durango, J., Velásquez, C.A., and Koll, G. 2004. The mineralogy and geochemistry of Cerro Matoso S.A. Ni laterite deposit, Montelíbano, Columbia. Economic Geology, Vol. 99: 1197-1213.

Golightly, J.P. 1981. Nickeliferous laterite deposits. Economic Geology $75^{\text {th }}$ Anniversary Volume: 710-735.

Golightly, J.P. 2010. Progress in understanding the evolution of nickel laterites. Society of Economic Geologists Special Publication, Vol. 15: 451-486.

Gorman, P.J., Kerrick, D.M., and Connolly, J.A.D. 2006. Modeling open system metamorphic decarbonation of subducting slabs. Geochemistry Geophysics Geosystems, Vol. 7, No. 4, pp. 21.

Halls, C. and Zhao, R. 1995. Listvenite and related rocks: Perspectives on terminology and mineralogy with reference to an occurrence at Cregganbaun, Co. Mayo, Republic of Ireland. Mineralium Deposita, Vol. 30: 303-313.

Hänchen, M., Prigiobbe, V., Storti, G., Seward, T.M., and Mazzotti, M. 2006. Dissolution kinetics of forsteritic olivine at $90-150^{\circ} \mathrm{C}$ including effects of the presence of $\mathrm{CO}_{2}$. Geochimica et Cosmochimica Acta, Vol. 70: 4403-4416.

Hansen, L.D., Dipple, G.M., Gordon, T.M., and Kellet, D.A. 2005. Carbonated serpentinite (listwanite) at Atlin, British Columbia: A geological analogue to carbon dioxide sequestration. Canadian Mineralogist, Vol. 43: 225-239.

Hanson, E.K., Moore, J.M., Bordy, E.M., Marsh, J.S., Howarth, G., and Robey, J.V.A. 2009. Cretaceous erosion in central South Africa: Evidence from upper-crustal xenoliths in kimberlite diatremes. South African Journal of Geology, Vol. 112: 125-140.

Holser, W., Schidlowski, M., Mackenzie, F., and Maynard, B. 1988. Geochemical cycles of carbon and sulfur. In: Bryan et al. (eds.) Chemical Cycles in the Evolution of the Earth. New York: John Wiley \& Sons, 105-173.

Kashiwagi, H., Ogawa, Y., and Shikazono, N. 2008. Relationship between weathering, mountain uplift, and climate during the Cenozoic as deduced from the global carbon-strontium cycle model. Palaeogeography, Palaeoclimatology, Palaeoecology, Vol. 270, No. 1-2: 139-149. 
Kelemen, P.B. and Matter, J.M. 2008. In situ carbonation of peridotite for $\mathrm{CO}_{2}$ storage. Proceedings of the National Academy of Sciences, Vol. 105, No.45: 17295-17300.

Kelemen, P.B., Matter, J.M., Streit, E.E., Rudge, J.F., Curry, W.B., and Blusztajn, J. 2011. Rates and mechanisms of mineral carbonation in peridotite: Natural processes and recipes for enhanced, in situ $\mathrm{CO}_{2}$ capture and storage. Annual Reviews of Earth and Planetary Sciences, Vol. 39: 545-576.

Kelley, D.S., Karson, J.A., Blackman, D.K., Früh-Green, G.L., Butterfield, D.A., Lilley, M.D., Olson, E.J., Schrenk, M.O., Roe, K.K., Lebon, G.T., and Rivizzigo, P. 2001. An off-axis hydrothermal vent field near the Mid-Atlantic Ridge at 30 degrees N. Nature, Vol. 412: 145-149.

Kerrick, D.M. and Connolly, J.A.D. 1998. Subduction of ophicarbonates and recycling of $\mathrm{CO}_{2}$ and $\mathrm{H}_{2} \mathrm{O}$. Geology, Vol. 26, No. 4: 375-378.

Klein, F. and Garrido, C.J. 2011. Thermodynamic constraints on mineral carbonation of serpentinized peridotite. Lithos, Vol. 126: 147-160.

Lackner, K.S., Wendt, C.H., Butt, D.P., Joyce, E.L., and Sharp, D.H. 1995. Carbon dioxide disposal in carbonate minerals. Energy, Vol. 20: 1153-1170.

Lackner, K.S. 2002. Carbonate chemistry for sequestering fossil carbon. Annual Review of Energy and the Environment, Vol. 27: 193-232.

Llorca, S. and Monchoux, P. 1991. Supergene cobalt minerals from New Caledonia. Canadian Mineralogist, Vol. 29: 149-161.

Luce, R. W., Bartlett, R. W., and Parks, G.A. 1972. Dissolution kinetics of magnesium silicates. Geochimica et Cosmochimica Acta, Vol. 36: 35-50.

Ludwig, K.A., Kelley, D.S., Butterfield, D.A., Nelson, B.K., and Früh-Green, G. L. 2006. Formation and evolution of carbonate chimneys at the Lost City hydrothermal field. Geochimica et Cosmochimica Acta, Vol. 70: 3625-2645.

Ludwig, K.A., Shen, C.-C., Kelley, D.S., Cheng, H., and Edwards, L.R. 2011. U-Th systematic and ${ }^{230} \mathrm{Th}$ ages of carbonate chimneys at the Lost City hydrothermal field. Geochmica et Cosmochimica Acta, Vol. 75: 1869-1888.

Matter, J.M. and Kelemen, P.B. 2009. Permanent storage of carbon dioxide in geological reservoirs by mineral carbonation. Nature Geoscience, Vol. 2: 837-841.

Moretti, I. and Turcotte, D.L. 1985. A model for erosion, sedimentation, and flexure with application to New Caledonia. Journal of Geodynamics, Vol. 3: 155-168. 
Nahon, D., Paquet, H., and Delvigne, J. 1982. Lateritic weathering of ultramafic rocks and the concentration of nickel in the western Ivory Coast. Economic Geology, Vol. 77: 1159-1175.

Nasir, S., Al-Sayigh, A.R., Al-Harthy, A., Al-Kirbash, S., Al-Jaaidi, O., Musllam, A., Al Mishwat, A., and Al-Bu'saidi, S. 2007. Mineralogical and geochemical characterization of listwaenite from the Semail Ophiolite, Oman. Chemie der Erde, Vol. 67, No. 3: 213-228.

O'Connor, W.K., Dahlin, D.C., Rush, G.E., Gerdemann, S.J., and Neilsen, D.N. 2004. Aqueous mineral carbonation. U.S. Department of Energy Final Report DOE/ARC-TR-04-002, U.S. Department of Energy Albany Research Center, Albany.

Oelkers, E. and Cole, D. 2008. Carbon dioxide sequestration: A solution to a global problem. Elements, Vol. 4: 305-310.

Oelkers, E., Gislason, S., and Matter, J. 2008. Mineral carbonation of $\mathrm{CO}_{2}$. Elements, Vol. 4: 333-337.

Orr, J., Fabry, V., Aumont, O., Bopp, L., Doney, S., et al. 2005. Anthropogenic ocean acidification over the twenty-first century and its impact on calcifying organisms. Nature, Vol. 437, No. 7059: 681-686.

Pokrovsky, O.S. and Schott, J. 2000. Kinetics and mechanism of foresterite dissolution at $25^{\circ} \mathrm{C}$ and $\mathrm{pH}$ from 1 to 12 . Geochimica et Cosmochimica Acta, Vol. 64, No. 19: 3313-3325.

Prigiobbe, V., Costa, G., Baciocchi, R., Hänchen, M., and Mazzotti, M. 2009. The effect of $\mathrm{CO}_{2}$ and salinity on olivine dissolution kinetics at $120^{\circ} \mathrm{C}$. Chemical Engineering Science, Vol. 64: 3510-3515.

Raymo, M. and Ruddiman, W. 1992. Tectonic forcing of late Cenozoic climate. Nature, Vol. 359, No. 6391: 117-122.

Rubey, W.W. 1951. Geologic history of seawater: An attempt to state the problem. Bulletin of the Geological Society of America, Vol. 62: 1111-1148.

Seifritz, W. 1990. $\mathrm{CO}_{2}$ disposal by means of silicates. Nature, Vol. 345: 486.

Spandler, C., Hermann, J., Faure, K., Mavrogenes, J.A., and Arculus, R.J. 2008. The importance of talc and chlorite "hybrid" rocks for volatile recycling through 
subduction zones: evidence from the high-pressure subduction mélange of New Caledonia. Contributions to Mineralogy and Petrology, Vol. 155: 181-198.

Staudigel, H., Hart, S., Schmincke, H.-U., and Smith, B. 1989. Cretaceous ocean crust at DSDP sites 417 and 418: carbon uptake from weathering versus loss by magmatic outgassing. Geochimica et Cosmochimica Acta, Vol. 53, No. 11: 3091-3094.

Summons, T.G., Green, D.C., and Everard, J.L. 1981. The occurrence of chromite in the Anderson Creek area, Beaconsfield, Tasmania. Economic Geology, Vol. 76: 505518.

Sundquist, E. T. 1993. The global carbon dioxide budget. Science, Vol. 259, No. 5097: 934-941.

Surour, A. A. and Arafa, E.H. 1997. Ophicarbonates: Calichified serpentinites fromGebel Mohagara, Wadi Ghadir area, Eastern Desert, Egypt. Journal of African Earth Sciences, Vol. 24: 315-324.

Trescases, J.J. 1973. Weathering and geochemical behavior of the elements of ultramafic rocks in New Caledonia. Bureau of Mineral Resources, Australia, Bulletin, Vol. 141: 149-163.

Trommsdorff, V. and Evans, B.W. 1977. Antigorite-ophicarbonates: Contact metamorphism in Valmalenco, Italy. Contributions to Mineralogy and Petrology, Vol. 62: 301-312.

Trommsdorff, V., Evans, B.W., and Pfeifer, H.R. 1980. Ophicarbonate rocks: Metamorphic reactions and a possible origin. Archives des Sciences Genève, Vol. 33: 361-364.

Van Herk, J., Pietersen, H.S., and Schuiling, R.D. 1989. Neutralization of industrial waste acids with olivine: The dissolution of forsteritic olivine at $40-70^{\circ} \mathrm{C}$. Chemical Geology, Vol. 76: 341-352.

Walker, J.C.G., Hays, P.B., and Kasting, J.F. 1981. A negative feedback mechanism for the long-term stabilization of Earth's surface temperature. Journal of Geophysical Research, Vol. 86, No. C10: 9776-9782.

Wilson, S.A., Raudseep, M., and Dipple, G.M. 2006. Verifying and quantifying carbon fixation in minerals from serpentine-rich mine tailings using the Rietveld method with X-ray powder diffraction data. American Mineralogist, Vol. 91, No. 8-9: $1331-1341$ 
Wilson, S.A., Raudsepp, M., and Dipple, G.M. 2009a. Quantifying carbon fixation in trace minerals from processed kimberlite: A comparative study of quantitative methods using X-ray powder diffraction data with applications to the Diavik Diamond Mine, Northwest Territories, Canada. Applied Geochemistry, Vol. 24, No. 12: 95-112.

Wilson, S.A., Dipple, G.M., Power, I.M., Thom, J.M., Anderson, R.G., Raudsepp, M., Gabites, J.E., and Southam, G. 2009b. Carbon dioxide fixation within minewastes of ultramafic-hosted ore deposits: Examples from the Clinton Creek and Cassiar chrysotile deposits, Canada. Economic Geology, Vol. 104, No. 1: 95-112.

Wogelius, R.A. and Walther, J.V. 1991. Olivine dissolution at $25^{\circ} \mathrm{C}$ : Effects of $\mathrm{pH}, \mathrm{CO}_{2}$, and organic acids. Geochimica et Cosmochimica Acta, Vol. 55: 943-954.

Yongue-Fouteu, R., Ghogomu, R.T., Penaye, J., Ekodeck, G.E., Stendal, H., and Colin, F. 2006. Nickel and cobalt distribution in the laterites of the Lomie region, south east Cameroon. Journal of African Earth Sciences, Vol. 45: 33-47. 


\title{
Chapter 2: ${ }^{14} \mathrm{C}$ Dating of Carbonate Alteration Products of Peridotite in the Samail Ophiolite, Sultanate of Oman
}

\begin{abstract}
:
Determining timescales of the formation and preservation of carbonate alteration products in peridotite is important in order to better understand the role of this potentially important sink in the global carbon cycle and also to evaluate the feasibility of using artificially-enhanced, in situ formation of carbonates in peridotite to mitigate the buildup of anthropogenic $\mathrm{CO}_{2}$ emissions in the atmosphere. Detailed ${ }^{14} \mathrm{C}$ dating as well as stable $\mathrm{C}$ and $\mathrm{O}$ isotope analyses were conducted on carbonate alteration products in the peridotite layer of the Samail Ophiolite, Sultanate of Oman. ${ }^{14} \mathrm{C}$ results obtained in this and previous (Clark and Fontes, 1990; Clark et al., 1992; Kelemen and Matter, 2008; Kelemen et al., 2011; Kelemen et al., unpublished data) studies indicate that surface travertines range in age from modern to $>45,000 \mathrm{yr} \mathrm{BP}$, indicating long-term deposition and preservation. Travertine deposition rates were $\sim 0.1-0.3 \mathrm{~mm} / \mathrm{yr}$ between $\sim 30,000$ 45,000 yr BP. Using an estimate of total travertine area from Kelemen and Matter (2008), this would result in a maximum of $\sim 1,000-3,000 \mathrm{~m}^{3} / \mathrm{yr}$ of travertine being deposited throughout the ophiolite during this time period. This travertine deposition would have sequestered a maximum of $\sim 1-3 \times 10^{6} \mathrm{~kg} \mathrm{CO}_{2} / \mathrm{yr} .{ }^{14} \mathrm{C}$ dating of Ca-rich carbonate veins (this study; Clark and Fontes, 1990; Kelemen and Matter, 2008; Kelemen et al., 2011; Kelemen et al., unpublished data) indicates that Ca-rich carbonate veins, which are associated with the surface travertine deposits, have ages ranging from $\sim 4,000-36,000 \mathrm{yr}$ BP (average: 15,000 yr BP). Mg-rich carbonate veins sampled on the natural peridotite weathering surface have ages ranging from $\sim 8,000-45,000 \mathrm{yr}$ BP (average: 35,000 yr
\end{abstract}


BP). Sampling from numerous locations (3 locations in this study and 10 locations in the previous studies) indicates that no carbonate veins from the natural peridotite weathering surface are older than the $\sim 50,000$ year dating limit of ${ }^{14} \mathrm{C}$. However, ${ }^{14} \mathrm{C}$ dating of $\mathrm{Mg}$ rich carbonate veins (this study; also Kelemen and Matter, 2008; Kelemen et al., 2011; Kelemen et al., unpublished) from three roadcut exposures (Qafeefah, Fanja, and AlWuqbah) indicates that a significant number of roadcut veins are ${ }^{14} \mathrm{C}$ dead $(>50,000 \mathrm{yr}$ BP). At Fanja Roadcut $\sim 75 \%$ of dated veins are ${ }^{14} \mathrm{C}$ dead while at Qafeefah and AlWuqbah Roadcuts $\sim 30 \%$ of veins are ${ }^{14} \mathrm{C}$ dead. A location-weighted average indicates that $\sim 40 \%$ of veins sampled at roadcuts are ${ }^{14} \mathrm{C}$ dead. An average including veins sampled at both roadcuts and outcrops indicates that overall $\sim 8 \%$ of $\mathrm{Mg}$-rich carbonate veins are ${ }^{14} \mathrm{C}$ dead. $\mathrm{Mg}$-rich carbonate veins are estimated to sequester on the order of $10^{7} \mathrm{~kg} \mathrm{CO}_{2} / \mathrm{yr}$ throughout the ophiolite.

\section{Introduction:}

The formation of carbonate minerals as alteration products in mafic and ultramafic rocks is a poorly-constrained sink in the global carbon cycle (e.g. Staudigel et al., 1989; Alt and Teagle, 1999; Dessert et al., 2001, 2003; Goldberg et al., 2008; Wilson et al., 2006; 2009a, b; Kelemen and Matter, 2008; Matter and Kelemen, 2009; Kelemen et al., 2011). While mafic rocks are more abundant than ultramafic rocks on Earth's surface, ultramafic rocks likely have faster carbonation rates than mafic rocks (O'Connor et al., 2004; Chizmeshya et al, 2007; Matter and Kelemen, 2009; Kelemen et al., 2011). Furthermore, studies of fully-carbonated peridotites, which are known as "listwanites", suggest that under certain conditions ultramafic rocks are capable of taking up 
significantly more $\mathrm{CO}_{2}$ than mafic rocks (e.g. Halls and Zhao, 1995; Hansen et al., 2005; Abkulut et al., 2006; Nasir et al., 2007; Kelemen et al., 2011).

The ultramafic rock peridotite is predominantly composed of the minerals olivine and pyroxene, which are far from equilibrium with $\mathrm{H}_{2} \mathrm{O}$ and $\mathrm{CO}_{2}$ on the Earth's surface and thus easily altered to hydrous silicates, Fe-oxides, and carbonates (e.g. calcite, magnesite, and dolomite). While natural carbonation of uplifted peridotite by alteration is commonly observed subaerially and on the seafloor (e.g. Trommsdorff and Evans, 1977; Trommsdorf et al., 1980; Ferry, 1995; Surour and Arafa, 1997; Kelley et al., 2001; FrühGreen et al., 2003; Ludwig et al., 2006; 2011; Kelemen and Matter, 2008; Matter and Kelemen, 2009; Kelemen et al., 2011), the natural rate of peridotite carbonation and therefore the rate of $\mathrm{CO}_{2}$ uptake via this alteration mechanism is poorly constrained (e.g. Wilson et al., 2006, 2009a, b; Kelemen and Matter, 2008; Kelemen et al., 2011). Determining the natural peridotite carbonation rate is critical for understanding the role of this potentially important sink in the global carbon cycle and is also an essential, but poorly constrained, parameter in calculations evaluating the viability of using artificiallyenhanced, in-situ alteration of peridotite to mitigate the buildup of anthropogenic $\mathrm{CO}_{2}$ in the atmosphere (e.g. Seifritz, 1990; Lackner et al., 1995; Lackner, 2002; Kelemen and Matter, 2008; Matter and Kelemen, 2009; Kelemen et al., 2011).

One way to determine timescales of natural carbonate formation in peridotite is to ascertain the formation ages of carbonate alteration products using techniques such as ${ }^{14} \mathrm{C}$ (e.g. Früh-Green et al., 2003; Kelemen and Matter, 2008; Wilson et al., 2009) and ${ }^{230} \mathrm{Th}$ dating (e.g. Ludwig et al., 2011). If the volume of carbonates being formed in dated 
deposits can be estimated, then rates of carbonate formation can be estimated (e.g. Kelemen and Matter, 2008; Wilson et al., 2009b).

An excellent location for investigating rates of carbonate formation in peridotite is the Samail Ophiolite, Sultanate of Oman, where abundant carbonate veins as well as calciterich travertines are found throughout the peridotite layer (e.g. Neal and Stanger, 1984; 1985; Clark and Fontes, 1990; Clark et al., 1992; Kelemen and Matter, 2008; Kelemen et al., 2011). Previous work has suggested that these veins have an average age of $\sim 26,000$ yr BP with none greater than $\sim 50,000 \mathrm{yr} B P$, the limit of the ${ }^{14} \mathrm{C}$ dating technique (Kelemen and Matter, 2008; Kelemen et al., 2011). These ${ }^{14} \mathrm{C}$ ages were used to calculate a rate of $\sim 4 \times 10^{7} \mathrm{~kg} \mathrm{CO}_{2} / \mathrm{yr}$ for natural $\mathrm{CO}_{2}$ sequestration in the peridotite layer of the ophiolite (Kelemen and Matter, 2008).

The goal of this study is to conduct detailed ${ }^{14} \mathrm{C}$ age dating as well as mineralogical and stable $\mathrm{C}$ and $\mathrm{O}$ isotope analysis to further assess the characteristics and rates of formation of carbonate alteration products in the peridotite layer of the Samail Ophiolite.

\section{Geologic Setting:}

\subsection{Geology of the Samail Ophiolite:}

The Samail Ophiolite (Figure 1) is one of the largest and best-exposed ophiolites in the world. Located in the deserts of the Sultanate of Oman and the United Arab Emirates, the Samail Ophiolite is part of a chain of related ophiolites found along the northern part of the Arabian plate. These ophiolites extend from Cyprus through Turkey and Iran down into northern Oman, then eastward through Pakistan, India, and Tibet 
(Ricou, 1971; Coleman, 1981; Lippard et al., 1986). They were emplaced onto the Arabian continental margin during the late Cretaceous, when the break-up of Gondwana led to the closing of the Tethyan Sea, a body of water that separated Gondwana from Eurasia (Coleman, 1981; Lippard et al., 1986).

The Samail Ophiolite consists of several uplifted, thrust-bounded blocks (nappes) of oceanic crust and mantle that were emplaced along with the underlying Hawasina pelagic sedimentary formation. These nappes consist of $\sim 4-7 \mathrm{~km}$ of crustal rocks (layered gabbros, sheeted dikes, and volcanics, including pillow lavas) and $\sim 8-12 \mathrm{~km}$ of upper mantle, primarily harzburgite (Glennie et al., 1973, 1974; Coleman, 1981; Lippard et al., 1986; Nicolas et al., 2000). The ophiolite is $\sim 50-100 \mathrm{~km}$ wide and extends for at least 500 km (Coleman, 1981; Nicolas et al., 2000). Gravity data indicate that the ophiolite could be as long as $\sim 800 \mathrm{~km}$ with a southeastern extension buried underneath Tertiary sediments (Nicolas et al., 2000).

The igneous rocks of the Samail Ophiolite were formed in a paleospreading center in the Tethyan Sea and during the emplacement of igneous crust over the Arabian continental margin, with igneous ages spanning $\sim 94-98 \mathrm{Ma}$ (Tilton et al., 1981; Rioux et al., 2008; Rioux et al., submitted 2012). Some geologists interpret these rocks as having formed at a typical, fast-spreading mid-ocean ridge with only minor modification during emplacement (e.g. Boudier and Coleman, 1981; Pallister and Hopson, 1981; Pallister and Knight, 1981; Boudier et al., 1988; Nicolas, 1989; Boudier and Nicolas, 2007). However, other geologists emphasize the presence of low- $\mathrm{Ti}$, high $\mathrm{Th} / \mathrm{Nb}$ geochemical signatures in some of the lavas - as well as orthopyroxene-bearing pyroxenite and gabbronorite 
cumulates-and suggest that the Samail spreading center formed above a northeastdipping subduction zone (e.g. Pearce et al., 1981; Searle and Malpas, 1980, 1982; Searle and Cox, 1999, 2002; Warren et al., 2003, 2005, 2007). In either geologic interpretation, Ar-Ar ages of hornblende in the metamorphic sole (Hacker, 1994) indicate that emplacement of the ophiolite began while some igneous activity was still taking place.

The Samail Ophiolite and the Hawasina were obducted $\sim 95-80$ Ma on top of autochthonous shelf carbonate rocks (the Hajar Supergroup) and parautochthonous continental slope carbonate rocks (the Sumeini Group), which rest on top of pre-Permian crystalline basement (Coleman, 1981; Lippard et al., 1986; Searle and Cox, 1999, 2002). The Samail and Hawasina nappes are locally overlain by reworked laterites and conglomerates, which in turn are overlain by younger shallow water marine limestones (Coleman, 1981). These overlying strata were deposited up until the late Miocene (Coleman, 1981). During the Pliocene, there was significant uplift and deformation of the Oman mountains, which resulted in domal uplifts at Jabal Akhdar and Saih Hatat (Glennie et al., 1974; Coleman, 1981). Despite this Pliocene tectonic uplift, the Samail Ophiolite nappes are remarkably well-preserved.

\subsection{Carbonate Alteration Products Formed in the Samail Ophiolite:}

There are four types of carbonates that form as a result of alteration of peridotite in the Samail Ophiolite: (1.) carbonate veins that form in situ in partially-hydrated (serpentinized) peridotite (Kelemen and Matter, 2008; Kelemen et al., 2011), (2.) travertine precipitated from high-pH springs (Neal and Stanger, 1984; 1985; Clark and Fontes, 1990; Kelemen and Matter, 2008; Kelemen et al., 2011), (3.) fully-carbonated 
peridotite rocks called "listwanite" that consist of magnesite or dolomite + quartz and formed at temperatures up to $\sim 20{ }^{\circ} \mathrm{C}$ (Stanger, 1985; Wilde et al., 2002; Nasir et al., 2007; Kelemen et al., 2011), and (4.) carbonate-rich cement found in peridotite breccias and alluvial conglomerates (Burns and Matter, 1995; Kelemen and Matter, 2008). The first two types of carbonate are the focus of this study.

Low-temperature alteration of peridotite in Oman has been proposed to occur in three steps, as shown in Figure 2 (Barnes et al., 1967, 1978; Barnes and O'Neil, 1969; Bruni et al., 2002; Cipolli et al., 2004; Kelemen et al., 2011). In the first step, meteoric water weathers partially-serpentinized peridotite in equilibrium with the atmosphere, forming $\mathrm{Mg}^{2+}-\mathrm{HCO}_{3}{ }^{-}$rich waters known as "Type I" waters. In the second step, these waters percolate deeper into the peridotite bedrock and are isolated from the atmosphere. These deeper waters precipitate $\mathrm{Mg}$-rich carbonates, such as magnesite and dolomite, and serpentine and clay are also formed. Precipitation of these minerals leads to an increase in $\mathrm{Ca}^{2+}$ in the fluid since $\mathrm{Ca}$ is largely excluded from these alteration minerals. $\mathrm{Ca}^{2+}$-rich waters, known as "Type II" waters, are depleted in magnesium and carbon and have pH of 10-12 (Neal and Stanger, 1983, 1984, 1985; Clark and Fontes, 1990) and Eh of approximately $-200 \mathrm{mV}$ (Neal and Stanger, 1983). In the third step, the Type II waters return to the surface as alkaline springs, where they rapidly take up atmospheric $\mathrm{CO}_{2}$ and precipitate abundant, calcite-rich travertine.

Calcite-rich travertines (Figure 3) associated with alkaline springs are found throughout the ophiolite. These springs are predominantly located in the peridotite layer, which is more brittle and fractured than the overlying gabbros and basalts (Neal and 
Stanger, 1984; 1985). Additionally, the alkaline springs are concentrated along the basal thrust of the ophiolite as well as at the crustal-mantle boundary (Neal and Stanger, 1984; 1985; Dewandel et al., 2003, 2004, 2005). These springs are generally alkaline to hyperalkaline with pH values of 10-12 (Clark and Fontes, 1990; Neal and Stanger, 1984; 1985; Paukert et al., submitted 2012). Thick travertine deposits (up to several meters, on average $\sim 1-2 \mathrm{~m}$ ) form on top of peridotite, which is highly-fractured with abundant carbonate-serpentine veins. Turquoise-blue alkaline pools lined with white carbonate precipitate are a feature of many alkaline springs. Browner, more weathered (and presumably older) travertine deposits are often found upslope and to the side of the actively-precipitating travertine deposits. Kelemen and Matter (2008) estimate that there are $\sim 45$ large travertine deposits in the entire Samail Ophiolite and that these deposits represent $\sim 10^{7} \mathrm{~m}^{3}$ of exposed travertine. Furthermore, based on measurements in outcrops immediately underlying travertine, they estimate that the travertine deposits are underlain by a $\sim 10 \mathrm{~m}$ thick peridotite layer with $\sim 5 \%$ Ca-rich carbonate veins, so the total volume of carbonate associated with the travertine deposits is probably 2.5 times the exposed volume.

Carbonate veins (Figure 3D and 4) form in situ in the mantle peridotite and, to a lesser extent, in the gabbro and basalt layers of the ophiolite. Near surface travertine deposits, carbonate veins are Ca-rich and similar in composition to the travertines. Carbonate veins sampled far from travertines are generally $\mathrm{Mg}$-rich and presumably formed in the deeper subsurface during the transformation of Type I waters to Type II waters. The Mg-rich carbonate veins are predominately composed of magnesite and 
dolomite and range in size from a few millimeters to several meters thick and can extend for hundreds of meters (Kelemen and Matter, 2008; Kelemen et al., 2011). The Mg-rich carbonate veins are often encompassed by or intergrown with serpentine veins. Based on data gathered at newly-formed roadcuts, Kelemen and co-workers estimate that carbonate veins comprise $\sim 1 \%$ of near-surface peridotite in the Samail Ophiolite (Kelemen and Matter, 2008; Kelemen et al., 2011).

\section{Sample Descriptions:}

Samples were collected at eight locations in the peridotite layer of the Samail Ophiolite (Figure 1): four locations of travertine deposition (Qafeefah ${ }^{1}$ Travertine, Misht $^{-1}$ Travertine, Wadi Uqaybah Travertine, and Wadi Sudari Travertine), three roadcuts exposing carbonate veins (Qafeefah Roadcut, Fanja Roadcut, and Al-Wuqbah Roadcut), and one campsite located on the natural peridotite weathering surface and containing abundant carbonate veins (Wadi Sudari Campsite). Sample descriptions are presented in Table 1. In addition to the new samples collected for this study, we also obtained replicate ${ }^{14} \mathrm{C}$ analyses for two previously dated carbonate veins. Descriptions and previous ${ }^{14} \mathrm{C}$ data for these two samples can be found in Kelemen and Matter (2008).

For the purposes of this study, we distinguish three types of carbonate veins (Figures 3D and 4). "Travertine veins" are Ca-rich (calcite or dolomite) veins that were sampled within 1-10 meters of surface travertine deposits (Figure 3D). Mg-rich carbonate veins are divided into "outcrop veins" (sampled from the natural peridotite

\footnotetext{
${ }^{1}$ Due to inconsistencies in transliteration from Arabic to English, Qafeefah has previously been spelled "Khafifah" or "Khafeefah" on maps and in geologic literature since at least 1981 (e.g. Pallister and Hopson, 1981; Gregory and Taylor, 1981; Neal and Stanger, 1985; Garrido et al., 2001; Van Tongeren et al., 2008). However, a more correct transliteration from the Arabic is "Qafeefah," in agreement with some but not all currently posted road signs in Oman.
} 
weathering surface; Figure 4, A and C) and "roadcut veins" (sampled at roadcuts, Figure 4, B and D). The roadcut veins are generally less weathered than the outcrop veins, which are often partially eroded.

At the four travertine locations sampled for this study, actively forming carbonate precipitates were collected from the surfaces and bottoms of hyperalkaline pools (Figure 3, A and B). These samples are distinguished as "recently-formed travertine precipitates." Travertine was also collected from travertine terraces $(\sim 1-2 \mathrm{~m}$ thick) and from incised channels located between the terrace formations (Figure 3, C and D). These two types of travetines are distinguished as "travertine terraces" and "channel-filling travertine." In order to estimate rates of travertine deposition, three travertine terraces were sampled at two field locations. At Misht Travertine, at least five distinct layers of a $\sim 2 \mathrm{~m}$ thick travertine terrace were carefully microsampled, and at Wadi Uqaybah Travertine, two $\sim 1$ m thick terraces were subsampled.

At many travertine locations, stalactite- and stalagmite-like travertine formations have developed in overhangs, such as underneath terrace formations. Note that since these formations are not found within true caves, they technically cannot be called speleothems even though morphologically they resemble speleothems. Therefore, these samples are distinguished as "pseudospeleothems." Two of the pseudospeleothems were dated: one actively-forming sample and one layered sample that was collected from a pile of pseudospeleothems "drops" which had fallen from a travertine overhang. Subsampling of the layered pseudospeleothem from Wadi Sudari was conducted in order to determine rates of travertine pseudospeleothem growth. 


\section{Methods:}

Select carbonate veins and travertines were made into chips using a jaw crusher and then purified using magnetic separation and hand-picking. Many of the carbonate veins consist of multiple minerals (e.g. magnesite, calcite, dolomite, and serpentine). Whenever possible, monomineralic (or nearly so) mineral separates were prepared, and their purity was evaluated by powder X-ray diffraction analysis at WHOI.

The travertine terraces and the speleothem-like travertines are often finelylaminated, heterogeneous on small length scales, and contain cross-cutting carbonate veins. Subsampling of the travertines was essential since a few centimeters of travertine deposition can represent several hundreds to thousands of years of travertine deposition. For this reason, travertine samples were carefully subsampled on a $\sim 1 \mathrm{~mm}$ scale. This subsampling was accomplished by milling approximately $50-100 \mathrm{mg}$ of carbonate material from thin sections using a semi-automated microsampler (see methods in Vadopalas et al., 2011).

All ${ }^{14} \mathrm{C}$ dating was conducted at the National Ocean Sciences Accelerator Mass Spectrometry Facility (NOSAMS) at Woods Hole Oceanographic Institution. For each sample, approximately $10-50 \mathrm{mg}$ of hand-picked or microsampled carbonate was weighed out for analysis. Immediately prior to dissolution, the samples were etched in $10 \%$ hydrochloric acid at room temperature to remove modern atmospheric $\mathrm{CO}_{2}$ adsorbed on mineral surfaces. Samples comprised primarily of calcite, dolomite, or aragonite were etched for $20-30$ seconds until approximately $50 \%$ of the material had dissolved. Samples comprised primarily of magnesite dissolved more slowly in 
hydrochloric acid, and these samples were etched in hydrochloric acid for several minutes until at least $10 \%$ of the material had dissolved.

The etched carbonate samples were dissolved in concentrated phosphoric acid on a vacuum line and $\mathrm{CO}_{2}$ gas was extracted using cryogenic traps. The $\mathrm{CO}_{2}$ was converted to graphite using either the standard acid dissolution method (Vogel et al., 1987; McNichol et al., 1995) or the sealed tube graphitization method (Xu et al., 2007; Burke et al., 2010). Initial ${ }^{14} \mathrm{C}$ dating was carried out using the standard acid dissolution method, which is time-consuming and expensive. In order to process more samples, additional ${ }^{14} \mathrm{C}$ analyses were carried out using a sealed tube graphitization method, which has a precision of 2-3\% (Xu et al., 2007; Burke et al., 2010), similar to the precision typically obtained for samples analyzed by standard dissolution method (Mark Roberts, personal communication, 2012).

The reproducibility of the ${ }^{14} \mathrm{C}$ measurements was evaluated through duplicate standard acid dissolution analyses on ten samples and through triplicate sealed tube graphitization analyses on five samples (see Table 2). The reproducibility of $F_{m}$ (Fraction modern ${ }^{14} \mathrm{C}$ ) for the standard acid dissolution method ranges from $<1$ to $9 \%$ with an average of $2 \%$. The reproducibility is the worst for a >modern sample (OM09-85C-MS) with very high $\mathrm{F}_{\mathrm{m}}$ and also for layered travertine samples (OM09-86C-MG and OM09106C-MS-B), which may not have been sub-sampled on a sufficiently fine scale. The variability of replicate ${ }^{14} \mathrm{C}$ analyses on layered travertines results in calibrated age differences of up to 2,000-3,000 years. The reproducibility of $F_{m}$ for the sealed tube graphitization method is much better: $1-3 \%$. The better reproducibility of the sealed tube 
graphitization analyses may reflect improvements in carbonate sub-sampling that were made after early initial ${ }^{14} \mathrm{C}$ analyses.

Two samples were analyzed by both the standard acid dissolution and the sealed tube graphitization methods to check the agreement of the methods. Sample OM09109C-MS, a channel-filling travertine, produced calibrated ${ }^{2}{ }^{14} \mathrm{C}$ ages of $5,867 \pm 25 \mathrm{yr} \mathrm{BP}$ and 5,543 \pm 78 yr BP (average: 5,705 yr BP) via the standard acid dissolution method and a calibrated ${ }^{14} \mathrm{C}$ age of $5,773 \pm 129 \mathrm{yr}$ BP via the sealed tube graphitization method. Sample OM09-47C-MS, a carbonate outcrop vein, produced calibrated ${ }^{14} \mathrm{C}$ ages of $28,559 \pm 239 \mathrm{yr}$ BP and $28,660 \pm 210 \mathrm{yr}$ BP (average: $28,610 \mathrm{yr}$ BP) via the standard acid dissolution method and a calibrated ${ }^{14} \mathrm{C}$ age of $30,526 \pm 263 \mathrm{yr}$ BP via the sealed tube graphitization method. Therefore, the agreement of the standard acid dissolution and sealed tube graphitization methods is excellent.

For the standard acid dissolution method, the $\mathrm{CO}_{2}$ gas was then combined with $\mathrm{H}_{2}$ gas and heated in the presence of an iron catalyst to make graphite. This graphite was then pressed into targets, which were analyzed for ${ }^{14} \mathrm{C} /{ }^{12} \mathrm{C}$ ratios on a $2.5 \mathrm{MV}$ tandetron accelerator mass spectrometer (AMS-1). For the sealed tube graphitization method, the $\mathrm{CO}_{2}$ was converted to graphite using a zinc reduction method (Xu et al., 2007; Burke et al., 2010). This graphite was stored in the sealed reaction tubes until immediately prior to pressing the graphite into targets. These targets were analyzed on a $500 \mathrm{kV}$ compact pelletron accelerator (CFAMS; Roberts et al., 2009).

\footnotetext{
${ }^{2}$ Ages were calibrated using the Calib 6.0 Program with the IntCa109 calibration curve (Reimer et al., 2009). See Section 5.2 below for more information.
} 
For both the standard acid dissolution and sealed tube graphite methods, primary and secondary standards and blanks were routinely measured. Secondary standards were analyzed with every 8-10 samples. The primary standard was NBS Oxalic Acid I (NIST$\mathrm{SRM}-4990, \mathrm{Fm}=1.0397)$, and the secondary standards were IAEA C-2 $\left(\mathrm{F}_{\mathrm{m}}=0.4114\right.$; Rozanski et al., 1992), TIRI-I $\left(\mathrm{Fm}=0.2524\right.$; Scott, 2003), and FIRI-C $\left(F_{\mathrm{m}}=0.1041\right.$, Boaretto et al., 2002). Blank corrections were made using analyses of the ${ }^{14} \mathrm{C}$ dead IAEA C-1 Carrara marble and Alfa Aeasar graphite (for the AMS machine background). Blanks for IAEA C-1 are $\mathrm{F}_{\mathrm{m}}=0.0017 \pm 0.009$ for the standard acid dissolution (equivalent to a ${ }^{14} \mathrm{C}$ age of 51,227 yr BP; $\mathrm{n}=112$; routine measurements by NOSAMS staff) and $\mathrm{F}_{\mathrm{m}}=$ $0.0023 \pm 0.0006$ (equivalent to a ${ }^{14} \mathrm{C}$ age of $48,664 \mathrm{yr} \mathrm{BP} ; \mathrm{n}=7$ ) for the sealed tube graphite method.

Carbon and oxygen isotopic analyses were conducted at the University of Michigan Stable Isotope Laboratory using a Finnigan MAT Kiel IV preparation device coupled directly to the inlet of a Finnigan MAT 253 triple collector isotope ratio mass spectrometer (see methods in Ivany et al., 2008). The precision and accuracy of the data were monitored through the daily analysis of a variety of powdered carbonate standards. Measured precision was maintained at better than $0.1 \%$ for both carbon and oxygen isotope compositions.

\section{Results:}

\subsection{Mineralogy:}

Results of X-ray diffraction of powdered samples are presented in Table 1. Mineral peaks were identified as "major" or "trace" but were not further quantified. 


\subsection{1: Travertines:}

The recently-formed travertine precipitates consist predominantly of calcite (4 of 7), and the rest consist predominantly of aragonite. The travertine terraces, travertine veins, and pseudospeleothems have very similar mineral compositions. They are composed of almost pure calcite with trace amounts of minerals such as clay, serpentine, and dolomite (common) and aragonite, brucite, and hydromagnesite (less common). In contrast to the recently-formed precipitates, none of the travertine terraces contain significant aragonite, probably because metastable aragonite has altered to calcite.

A few travertine samples (OM09-32C-MG-BROWN, OM10-28C-MG-BROWN, OM10-37C-MG, OM09-108C-MG) contain dolomite as a major mineral phase. Samples OM09-32C-MG-BROWN and OM10-28C-MG-BROWN are brown, more weathered, more porous separates that were sampled adjacent to white, less weathered, denser separates (samples OM09-32C-MG-WHITE and OM10-28C-MG-WHITE) from the same layers of the same travertine terraces. While the brown separates contain significant dolomite (and also trace aragonite in the case of OM10-32C-MG), the white separates are almost pure calcite. This may indicate that these brown separates have been affected by diagenesis. Sample OM10-37C-MG is a channel-filling travertine while sample OM09108C-MS is a travertine vein. Travertine vein sample OM09-84C-MS contains both calcite and brucite as major minerals and also contains trace hydromagnesite. Finally, two travertine samples (OM10-78C-MG-\#1 and OM10-78C-MG-\#2) from the bottom of a travertine terrace at Wadi Uqaybah consist primarily of halite with only trace calcite and 
dolomite. The origin of the halite is ambiguous but may have originated from evaporated wadi water that ran along the base of the travertine terrace.

\subsection{2: Mg-Rich Carbonate Veins:}

The majority of the Mg-rich carbonate veins consist predominantly of magnesite with trace clays and sometimes trace dolomite, calcite, and serpentine. The magnesiterich veins are opaque, bright white, and blocky (Figure 4C). However, some of the Mgrich carbonate veins, particularly those sampled at roadcuts, consist primarily of dolomite rather than magnesite (Figure 4D). Dolomite (which is more soluble than magnesite) is likely better preserved at roadcuts, where the carbonate veins are generally lessweathered. The dolomite veins are often (though not exclusively) clear rather than opaque. In the case of one layered vein (OM10-48C-MG) on the surface of Al-Wuqbah Roadcut, the upper layer is opaque white while the lower layer is clear. Both layers consist primarily of dolomite. However, the lower layer also contains magnesite and calcite while the upper layer contains trace aragonite and clay. A few Mg-rich veins (OM10-83C-MG, OM10-84C-MG, OM10-51C-MG) also contain significant calcite in addition to magnesite and/or dolomite.

\subsection{Distribution of Carbonate ${ }^{14} \mathrm{C}$ Ages:}

Results of the ${ }^{14} \mathrm{C}$ analyses are reported as Fraction Modern $\left(\mathrm{F}_{\mathrm{m}}\right)$ and as both uncalibrated and calibrated ${ }^{14} \mathrm{C}$ ages in years $\mathrm{BP}$ in Table 2. For reference, previously unpublished ${ }^{14} \mathrm{C}$ data of Kelemen et al. and also replicate analyses (done in 2009) of two Kelemen and Matter (2008) samples are also given in Table 2. Fraction Modern $\left(\mathrm{F}_{\mathrm{m}}\right)$ is the deviation of a sample from "modern" where modern is defined as $95 \%$ of the 
radioactive carbon concentration (in AD 1950) of NBS Oxalic Acid I normalized to $\delta^{13} \mathrm{C}_{\mathrm{VPDB}}=-19 \%$ (Olsson, 1970). The $\mathrm{F}_{\mathrm{m}}$ results were calculated using the internationally accepted modern value of $1.176 \times 10^{-12}$ (Karlen et al., 1964). The sample $F_{m}$ was also corrected to a $\delta^{13} \mathrm{C}_{\mathrm{VPDB}}$ value of $-25 \%$. For AMS-1 measurements, this correction was done using externally measured $\delta^{13} \mathrm{C}$. For CFAMS measurements, this correction was done using concurrently measured ${ }^{13} \mathrm{C} /{ }^{12} \mathrm{C}$ ratios. The uncalibrated ${ }^{14} \mathrm{C}$ ages were calculated from $\mathrm{F}_{\mathrm{m}}$ using 5568 years as the half-life of radiocarbon and following the convention in Stuiver and Polach (1977) and Stuiver (1980). Calibrated ${ }^{14} \mathrm{C}$ ages were obtained using the Calib 6.0 Program with the IntCal09 calibration curve (Reimer et al., 2009).

\subsection{1: Travertines:}

Five (out of seven) recently-formed travertine precipitates as well as three channel-filling travertines from Wadi Sudari Travertine have $F_{m}>1.00$, which indicates that the samples were deposited after 1950 (Table 2). Nuclear bomb tests in the 1950s and 1960 s added considerable "bomb ${ }^{14} \mathrm{C}$ " to the troposphere. In the northern hemisphere, the amount of ${ }^{14} \mathrm{C}$ in the troposphere reached a peak of $\sim 100 \%$ greater than the 1950 value in 1963, the year of the Nuclear Test Ban Treaty (e.g. Hua and Barbetti, 2004). Since 1963, tropospheric levels of ${ }^{14} \mathrm{C}$ have been gradually falling, and today levels in the northern hemisphere troposphere are $F_{m}=\sim 1.05-1.06$ (e.g. Hua and Barbetti, 2004; Graven et al., 2012). The $F_{m}$ value of the troposphere in northern Oman has not been measured recently. Clark et al. (1992) measured tropospheric values of $F_{m}=$ 1.17 and $F_{m}=1.19$ for northern Oman, but these values have undoubtedly fallen 
significantly since 1992 . We measured a value of $F_{m}=1.09$ in the bark of a small shrub sampled at Wadi Uqaybah (see Table 2), but this shrub bark may have been growing for several years. Therefore, we assume that the current $F_{m}$ of the troposphere in northern Oman is within the global northern hemisphere range of $\mathrm{F}_{\mathrm{m}}=\sim 1.05-1.06$.

The seven recently-formed travertine precipitates cover a range of $F_{m}$ values. At Qafeefah Travertine, precipitates sampled in 2009 have values of $F_{m}=0.9710 \pm 0.0034$ (OM09-6COPS-MS) and $\mathrm{F}_{\mathrm{m}}=0.9569 \pm 0.0052$ (OM09-7COPS-MS) while precipitates sampled in 2010 have values of $F_{m}=1.0064 \pm 0.0047$ (OM10-1COPS-MG) and $F_{m}=$ $1.0022 \pm 0.0063(\mathrm{OM} 10-2 \mathrm{COPS}-\mathrm{MG})$. At Misht Travertine, a precipitate sampled in 2010 has $F_{m}=1.0036 \pm 0.0052$ (OM10-6COPS-MG). At Wadi Sudari Travertine, a precipitate sampled in 2009 has $\mathrm{F}_{\mathrm{m}}=1.0137 \pm 0.0031$ (OM09-8COPS-MS). Finally, at Wadi Uqaybah Travertine a precipitate sampled in 2009 has $F_{m}=1.0046 \pm 0.0035$ (OM09-10COPS-MS). Three other travertine samples have $F_{m}>1.00$. Two channelfilling travertines from Wadi Sudari have $F_{m}=1.0434 \pm 0.0031(\mathrm{OM} 09-81 \mathrm{C}-\mathrm{MS})$ and $\mathrm{F}_{\mathrm{m}}$ $=1.0026 \pm 0.0031$ (OM09-89C-MS). Finally, a pseudospleothem from Wadi Sudari Travertine has $\mathrm{F}_{\mathrm{m}}=1.5064 \pm 0.0056$ (OM09-85C-MS). This pseudospeleothem was observed to be actively forming from a drip originating from an overhanging travertine terrace.

The $F_{m}$ values measured in the recently-formed travertine precipitates suggest that the majority of carbon incorporated into these travertines is atmospheric in origin. However, all seven of the recently-formed travertine precipitates have $F_{m}$ lower than the current $\sim 1.05-1.06$ value of the Northern Hemisphere troposphere. Therefore, they must 
also be incorporating a small amount of older, non-atmospheric carbon, possibly carbon contributed from older travertines or carbonate veins that are weathered by the Type II hyperalkaline waters. The $F_{m}=1.0026 \pm 0.0031$ value measured in channel-filling travertine OM09-89C-MS also indicates the recycling of a small amount of older carbon in this sample. The $F_{m}=1.0434 \pm 0.0031$ value measured in channel-filling travertine OM09-81C-MS is more similar to the current $F_{m}$ of the atmosphere. However, this sample could have been precipitated several years ago, when $F_{m}$ was higher. Therefore, the contribution of some recycled carbon in this sample cannot be ruled out. The high $F_{m}$ $=1.5064 \pm 0.0056$ observed in the currently-forming pseudospeleothem sample (OM09$85 \mathrm{C}$-MS) is notable because this value is significantly higher than current tropospheric $F_{m}$ values. Therefore, this sample must reflect re-precipitation of older travertine that was precipitated when tropospheric $\mathrm{F}_{\mathrm{m}}$ was higher.

Clark et al. (1992) and Kelemen et al. (Kelemen and Matter, 2008 and Kelemen et al., unpublished) also measured $F_{m}$ values in recently-formed travertine precipitates. Clark et al. (1992) measured $F_{m}=1.1308 \pm 0.125$ and $F_{m}=1.1308 \pm 0.0082$ in two precipitates from the Nizwa area in 1992. Kelemen et al. measured $F_{m}=1.1296 \pm 0.0043$ in a travertine from Wadi Mahram in 2007 (Kelemen and Matter, 2008) and $F_{m}=0.9681$ \pm 0.0038 in a travertine from Kharma in 2008 (Kelemen et al., unpublished; also see Table 2). The higher $F_{m}$ values in the Clark et al. samples likely reflect the higher atmospheric $F_{m}$ values in 1992. The $F_{m}$ value measured in the Wadi Mahram sample is higher than tropospheric $F_{m}$ in 2007 and could reflect the recycling of some older 
travertines with a higher bomb ${ }^{14} \mathrm{C}$ signature. The $\mathrm{F}_{\mathrm{m}}$ value measured in the Kharma sample indicates a small amount of older (pre-bomb) carbon in this precipitate.

Overall, the young ages of the recently-formed travertine precipitates indicate that the majority of $\mathrm{CO}_{2}$ being incorporated into travertines is atmospheric in origin. However, the variations observed in the $F_{m}$ values measured in young travertines suggest that there is some open system behavior and recycling of carbon during travertine precipitation. Detailed study of the $\mathrm{F}_{\mathrm{m}}$ of young travertines (precipitates, channel-filling travertines, and currently-forming pseudospeleothems) could be a fruitful area of future research that could provide more information about open-system behavior during travertine formation.

Samples from travertine terraces $(\mathrm{n}=28)$ range in age from $\sim 8,000-45,000 \mathrm{yr}$ BP with an average age of $\sim 31,000 \mathrm{yr}$ BP (Figures 5 and 6). However, there are some sampling biases in the travertine data. Most of the samples were collected from the tops of travertine terraces, which are easier to sample and generally younger than travertine sampled from the middle or bottom of travertine terraces. Additionally, three travertine terraces from two field locations were subsampled. For example, 12 (out of 16) of the travertine terrace samples dated from Misht Travertine were collected from the same $\sim 2$ $m$ thick terrace exposure (Figure 7). The oldest travertine in the Misht terrace sequence $(\sim 45,000 \mathrm{yr} \mathrm{BP})$ is found at the bottom of the terrace, just above the altered peridotite bedrock. The upper layer of the Misht terrace sequence has an age of $29,000 \mathrm{yr}$ BP. Within the sequence, three brown, porous, travertine samples (OM10-32C-MS-\#8, OM10-32C-MG-\#10, and OM10-32C-MG-\#11) - all located on the outer edge of the 
terrace exposure - have anomalously young ${ }^{14} \mathrm{C}$ ages inconsistent with their stratigraphic positions. These three samples also have anomalously heavier stable $\mathrm{C}$ and $\mathrm{O}$ isotope values relative to adjacent samples and likely have been affected by weathering and/or diagenesis that has partially or fully re-set the ${ }^{14} \mathrm{C}$ ages. At Wadi Uqaybah Travertine, two $\sim 1 \mathrm{~m}$ thick terraces were subsampled (Figure 8 ). The first terrace ranges in age from $\sim 42,000-38,000$ yr BP (4 subsamples) with one sample having an anomalously old age compared to the samples above and below it. The second terrace ranges in age from $\sim 30,000-34,000$ yr BP (2 subsamples).

Seven channel-filling travertines were analyzed for ${ }^{14} \mathrm{C}$ (Figures 5 and 6). Two samples have $\mathrm{F}_{\mathrm{m}}>1$ while the remaining samples range in age from $\sim 2,200-8,000 \mathrm{yr}$ BP with an average age of $\sim 5,000 \mathrm{yr} \mathrm{BP}$. As previously discussed, the actively-forming pseudospeleothem (OM09-85C-MS) has $\mathrm{F}_{\mathrm{m}}>1$. The layered subsamples from the pseudospeleothem drop sample (OM09-76C-MS-A, -B, and -C), which were sampled from the edges and center of the $\sim 5 \mathrm{~cm}$ thick sample, are age progressive with ages of $34,289,39,166$, and 43,455 yr BP respectively (Figure 9).

The range of ${ }^{14} \mathrm{C}$ ages for surface travertine deposits falls within the range of ages previously obtained for Samail travertines (Figure 6). Clark and Fontes (1990) obtained ${ }^{14} \mathrm{C}$ ages for travertine deposits near the village of Nizwa that ranged from $\sim 1,000$ to $>50,000 \mathrm{yr} \mathrm{BP}$ (one sample was $>50,000 \mathrm{yr} \mathrm{BP}$ ) with an average age of $\sim 14,000 \mathrm{yr} \mathrm{BP}$ while Kelemen et al. (Kelemen and Matter, 2008; Kelemen et al., 2011; Kelemen et al., unpublished) obtained ${ }^{14} \mathrm{C}$ ages for travertines that ranged from $\sim 1,500$ to $>45,000$ yr BP with an average age of $\sim 30,000 \mathrm{yr}$ BP. 
Ca-rich veins associated with travertine deposits (Figure 10) are generally younger than the Mg-rich veins sampled at roadcuts and outcrops far from travertines (Figures 11, and 12). The travertine vein samples $(n=5)$ have ages ranging from $\sim 5,000$ $24,000 \mathrm{yr}$ BP with an average age of $\sim 14,000 \mathrm{yr} \mathrm{BP}$. This range of ages is similar to those previously obtained by Clark and Fontes (1990), who obtained ages of $\sim 6,500-36,000 \mathrm{yr}$ BP (average: $\sim 16,000$ yr BP), and by Kelemen and co-workers (Kelemen and Matter, 2008; Kelemen et al., 2011; Kelemen et al., unpublished data), who obtained ages of $\sim 7,000-35,000$ yr BP (average: 18,000 yr BP).

\subsection{2: Mg-Rich Carbonate Veins:}

Mg-rich carbonate outcrop veins $(n=7)$ sampled from three locations range in age from $\sim 29,000-43,000 \mathrm{yr}$ BP with an average age of $\sim 38,000$ years BP (Figure 11). These ages are at the older end of the age range for outcrop veins of $\sim 8,000-45,000 \mathrm{yr} \mathrm{BP}$ (average: $\sim 34,000$ yr BP; $\mathrm{n}=21$ ) previously obtained by Kelemen and co-workers (Kelemen and Matter, 2008; Kelemen et al., 2011; Kelemen et al., unpublished data.) One outcrop vein (actually, a vein sampled from the bottom of a Falaj or water channel) analyzed by Kelemen and Matter (2008) was ${ }^{14} \mathrm{C}$ dead.

Mg-rich roaduct veins $(n=24)$ sampled from three locations show a significantly different range of ages (Figure 12). While half $(n=12)$ of the roadcut veins range in age from $\sim 22,000-45,000 \mathrm{yr}$ BP with an average age of $\sim 34,000 \mathrm{yr}$ BP (similar to the outcrop veins), the other half are ${ }^{14} \mathrm{C}$ dead. However, the ${ }^{14} \mathrm{C}$ dead veins are not evenly distributed amongst the three roadcut sampling locations. The majority are located at Fanja Roadcut, where 7 out of 9 individual veins are ${ }^{14} \mathrm{C}$ dead, and an eighth vein has an age of 44,497 $\mathrm{yr}$ 
$\mathrm{BP}$, which is very close to the limit of ${ }^{14} \mathrm{C}$ dating. At Qafeefah Roadcut, 3 out of 8 individual veins are ${ }^{14} \mathrm{C}$ dead. Previously, six veins were dated from Qafeefah Roadcut, and two of these veins were ${ }^{14} \mathrm{C}$ dead while the remaining four veins ranged in age from $\sim 13,000-43,000 \mathrm{yr}$ BP with an average age of $\sim 32,000 \mathrm{yr}$ BP (Kelemen and Matter, 2008; Kelemen et al., 2011; Kelemen et al., unpublished data).

At Al-Wuqbah Roadcut, 2 out of 5 individual veins are ${ }^{14} \mathrm{C}$ dead. One of the two ${ }^{14} \mathrm{C}$ dead samples comes from a heterogeneous vein that was subsampled into an opaque, chalky dolomite fraction (facing the roadcut's outer surface) and a clear, hard dolomite fraction (underneath). The opaque layer has an age of $\sim 33,000 \mathrm{yr}$ BP while the clear layer is ${ }^{14} \mathrm{C}$ dead. Two possible explanations for the age differences in this heterogeneous vein are: (1.) the outer, opaque layer represents a part of the vein which has been affected by dissolution/re-precipitation, or (2.) the outer, opaque layer represents a part of the vein which grew at a later time, thus indicating that veins can form over long time periods.

\subsection{Stable Isotope Analyses:}

The results of $\delta^{13} \mathrm{C}$ and $\delta^{18} \mathrm{O}$ analyses of Samail carbonates are presented in Table 2 and plotted in Figure 13. Samail carbonates cover a wide range of carbon and oxygen isotope compositions, ranging from approximately -27 to $+5 \%$ VPDB for $\delta^{13} \mathrm{C}$ and approximately +13 to $+40 \%$ VSMOW for $\delta^{18} \mathrm{O}$. The Mg-rich carbonate veins are generally isotopically heavier (Average $\delta^{13} \mathrm{C}:-5.22 \% \pm 3.02 \%$ ( $1 \sigma$ ) VPDB; Average $\delta^{18} \mathrm{O}: 32.46 \%$ o $3.71 \%$ o $(1 \sigma)$ VSMOW) than the surface travertines (Average $\delta^{13} \mathrm{C}$ : $-13.96 \%$ $\pm 7.27 \%$ ( $(1 \sigma)$ VPDB; Average $\delta^{18} \mathrm{O}: 22.80 \% \pm \pm 5.06 \%$ ( $\left.1 \sigma\right)$ VSMOW). There is no significant difference between the isotopic composition of Mg-rich outcrop 
(Average $\delta^{13} \mathrm{C}:-3.54 \%$ $\pm 2.73 \%$ o $(1 \sigma)$ VPDB; Average $\delta^{18} \mathrm{O}: 33.27 \%$ $\pm 4.24 \%$ o $(1 \sigma$ ) VSMOW) versus roadcut veins (Average $\delta^{13} \mathrm{C}:-6.53 \%$ $\pm 2.40 \%$ ( $\left.1 \sigma\right)$ VPDB; Average $\delta^{18} \mathrm{O}: 31.83 \%$ o $\pm 3.10 \%(1 \sigma)$ VSMOW). The travertines have roughly correlated $\delta^{13} \mathrm{C}$ and $\delta^{18} \mathrm{O}$, which extend down to light values that are unusual for inorganic carbonates (see the extensive discussion on this in Kelemen et al., 2011). The travertine veins also tend to have heavier stable isotope values (Average $\delta^{13} \mathrm{C}:-5.06 \%$ 年 $4.84 \%$ o $(1 \sigma)$ VPDB; Average $\delta^{18} \mathrm{O}: 30.20 \%$ o $\pm 4.27 \%$ o $(1 \sigma)$ VSMOW) than the surface travertines. A few of the surface travertines do extend to heavier values. However, two of the surface travertine samples with heavier $\delta^{13} \mathrm{C}$ and $\delta^{18} \mathrm{O}$ isotopic ratios are OM09-32C-MS-BROWN $\left(\delta^{13} \mathrm{C}=-2.61 \%\right.$ o VPDB and $\delta^{18} \mathrm{O}=+30.21 \%$ VSMOW $)$ and OM10-28C-MG-BROWN $\left(\delta^{13} \mathrm{C}=+1.34 \%\right.$ VPDB and $\delta^{18} \mathrm{O}=+34.45 \%$ VSMOW) are brown, porous, weathered travertines that have anomalously young ${ }^{14} \mathrm{C}$ ages relative to adjacent samples. The stable isotope values of these samples may have been affected by weathering and/or diagenesis.

\section{Discussion:}

\section{1: Temporal and Spatial Trends in Carbonate Formation:}

\subsection{1: Surface Travertine Deposition and Preservation:}

The ages of the surface travertines indicate that travertine deposition and preservation has been ongoing for at least the past 50,000 yr BP. The long-term preservation of the travertines is remarkable and may be a consequence of Oman's arid climate. Except for a period of increased rainfall from $\sim 8,000-9,000$ yr BP during the early Holocene humid period, Oman's climate has been fairly arid over the past 50,000 yr BP (Burns et al., 2001; Fleitmann et al., 2003a, 2003b, 2007; Fuchs and Buekert, 2008). 
Oman's climate has become increasingly arid since the end of the Holocene humid period $\sim 8,000 \mathrm{yr} \mathrm{BP}$ and is likely more arid today than it was prior to the humid period (Burns et al., 2001; Fleitmann et al., 2003a, b, 2007; Fuchs and Buekert, 2008).

Based on ${ }^{14} \mathrm{C}$ ages and also the heavier stable $\mathrm{C}$ and $\mathrm{O}$ isotopic ratios of surface travertines and travertine veins, Clark and Fontes (1990) argued for a pluvial period prior to $19,000 \mathrm{yr}$ BP and a second pluvial period (corresponding to the early Holocene humid period) from 6,500-12,500 yr BP, and for hyperarid climate from 16,300-19,000 yr BP and again from $6,100 \mathrm{yr} \mathrm{BP}$ to the present day with transitional periods in-between. Additional ${ }^{14} \mathrm{C}$ ages for travertines obtained in this study and by Kelemen et al. (Kelemen and Matter, 2008; Kelemen et al., 2011; Kelemen et al., unpublished) fill in the gaps in the Clark and Fontes (1990) travertine dataset and suggest that travertine formation has been continuous over the past 45,000 years or at least that there are insufficient data to detect periods of rapid versus slow travertine deposition (Figure 6).

Although the overall ${ }^{14} \mathrm{C}$ age distributions do not support climate-related episodic deposition of travertines, there is field evidence that suggests that travertine deposition has been episodic, and the travertines have experienced erosion. For example, at the four travertine locations investigated in this study, the travertine terraces are brown and weathered, and channels have cut through both the terraces as well as up to 1-2 $\mathrm{m}$ of the underlying peridotite bedrock (Figures $3 \mathrm{C}$ and $3 \mathrm{D}$ ). The travertine terraces at these locations are primarily $\sim 30,000-45,000$ yr $\mathrm{BP}$ in age, and deposition of the modern travertines at these locations primarily occurs in the bottoms of the incised channels. There may have been a time period of widespread travertine deposition that produced the 
1-2 $\mathrm{m}$ thick travertine terraces that was followed by a period or event of travertine erosion, which eroded the tops of some of the terraces and also created the incised channels. Subsequently, there was renewed deposition of travertine, primarily in the incised channels. However, there is not presently enough data to evaluate the specific nature and timing of episodic travertine deposition and erosion.

Based on the thickness and dating of layers within travertine terraces (Figures 7 and 8 ), we have calculated deposition rates of $\sim 0.1 \mathrm{~mm} / \mathrm{yr}$ for the time period $\sim 29,000$ to $\sim 45,000 \mathrm{yr}$ BP for the Misht Travertine and $\sim 0.3 \mathrm{~mm} / \mathrm{yr}$ for the time period $\sim 30,000$ to $\sim 42,000$ yr BP for the Wadi Uqaybah Travertine. In addition, we have calculated deposition rates of $\sim 0.005 \mathrm{~mm} / \mathrm{yr}$ for pseudospeleothem growth at Wadi Sudari Travertine (Figure 9). The precipitation rates for the pseudospeleothem are likely slower because it was formed through dripping and evaporation rather than by direct precipitation in an alkaline pool. Compared with deposition rates for other types of travertines (Table 4), the Samail travertines deposit relatively slowly. Thermogene (hot spring) travertines have much faster precipitation rates of $1-1000 \mathrm{~mm} / \mathrm{yr}$ (Pentecost, 2005). However, meteogene (meteoric water) travertine precipitation rates range from 0.04-48 mm/yr (Pentecost, 2005). The Samail travertine precipitation rates are most similar to speleothem (cave) travertine deposition rates, which range from $0.002-0.90$ $\mathrm{mm} / \mathrm{yr}$ (Pentecost, 2005).

\subsection{2: Mg-Rich Carbonate Veins:}

The older ages of the Mg-rich carbonate veins relative to the Ca-rich carbonate veins are consistent with the concept that Type I waters deposit Mg-rich carbonates in the 
subsurface, become enriched in Ca as they evolve into Type II waters, and then deposit Ca-rich travertines at the surface (see Section 2.2). The Ca-rich carbonate veins are sampled near, and often underneath, layered travertine deposits and likely serve as feeder veins formed in the pathways of Type II waters towards the surface and/or form by downward migration of Type II waters into cracks underlying the travertine terraces.

The presence of both older $\left({ }^{14} \mathrm{C}\right.$ dead $)$ and younger Mg-rich carbonate veins at roadcuts may reflect open-system behavior. In order for the Mg-rich carbonate vein ages to be interpreted as formation ages, the assumption must be made that the carbon incorporated into the veins originated as atmospheric carbon. However, veins might undergo post-crystallization, open-system exchange with relatively ${ }^{14} \mathrm{C}$-rich fluids, and this exchange could produce apparent ${ }^{14} \mathrm{C}$ ages which are younger than the initial formation ages. Alternatively, if the veins incorporated significant amounts of older carbon, then the apparent ${ }^{14} \mathrm{C}$ ages may be older than the formation ages. There are two possible sources of older carbon that could be incorporated into veins as they precipitate: (1.) older veins, which could dissolve (fully or partially) and re-precipitate, thereby recycling older carbon and (2.) the Tertiary to Precambrian carbonates (and also sandstones and siltstones with a carbonate component) located in the northern Oman mountains (e.g. Coleman et al., 1981; Weyhenmeyer, 2000), which could contribute much older carbon if vein-forming fluids percolated through them.

Two lines of evidence suggest that the Samail carbonates have not incorporated significant older carbonate material from the Tertiary to Precambrian formations. First, the $\delta^{13} \mathrm{C}, \delta^{18} \mathrm{O}$, and ${ }^{87} \mathrm{Sr} /{ }^{86} \mathrm{Sr}$ values of the Samail carbonates are distinct from values 
measured in the Tertiary to Precambrian limestones, dolostones, sandstones, and siltstones and also from the carbonate matrices of alluvial conglomerates from Sur (Figure 8; sediment data from Weyhenmeyer, 2000, Burns and Matter, 1995; also see Kelemen et al., 2011, Supplementary Table T3 and Supplementary Figure S3). The Sur conglomerate matrices were derived from waters which percolated through Maastrichtian to Tertiary carbonates and also through some Hawasina outcrops (Burns and Matter, 1995). While both the Sur samples and the Samail carbonates cover a wide range of $\delta^{13} \mathrm{C}$ and $\delta^{18} \mathrm{O}$ values, which are positively correlated, the $\delta^{13} \mathrm{C}$ and $\delta^{18} \mathrm{O}$ trend for the Sur carbonates is higher and is more closely aligned with the $\delta^{13} \mathrm{C}$ and $\delta^{18} \mathrm{O}$ values measured in the Tertiary to Precambrian carbonate and sandstone/siltstone rocks. Second, fluids sampled from the Samail peridotite have young ${ }^{14} \mathrm{C}$ ages (Matter et al., 2012), indicating that they contain mostly atmospheric $\mathrm{CO}_{2}$. Furthermore, we do not observe significant amounts of older carbon in recent travertine precipitates from alkaline spring water (see Section 5.2.1). Therefore, we interpret the roadcut vein age distribution as representing multiple generations of carbonate vein formation, although the distribution of the apparent ages may have been affected by some open-system (dissolution and reprecipitation) behavior.

Approximately $50 \%$ of the $\mathrm{Mg}$-rich carbonate roadcut veins analyzed in this study formed $>50,000 \mathrm{yr}$ BP. However, it is important to account for the bias introduced by the large number of ${ }^{14} \mathrm{C}$ dead veins from Fanja Roadcut, as discussed in Section 5.2.1. A locality-weighted average of veins dated from the three roadcut locations provides a lower value of $40 \%{ }^{14} \mathrm{C}$ dead veins. Furthermore, despite analysis of numerous $(n=28)$ 
Mg-rich carbonate outcrop veins from 13 locations in the ophiolite (this study; also Kelemen and Matter, 2008; Kelemen et al., 2011; Kelemen et al., unpublished data), no ${ }^{14} \mathrm{C}$ dead samples have been observed on the natural peridotite weathering surface. An average which includes both outcrop and roadcut veins suggests that overall $\sim 8 \%$ of $\mathrm{Mg}$ rich carbonate veins are ${ }^{14} \mathrm{C}$ dead.

\subsection{3: Isotopic Variability:}

The surface travertines (Figure 14) exhibit generally lighter $\delta^{13} \mathrm{C}(-27$ to $1.34 \%$ VPDB) and $\delta^{18} \mathrm{O}$ (14 to $34 \%$ VSMOW) values than the Mg-rich carbonate veins (Figure 15). The young travertines ( $<1000 \mathrm{yr} \mathrm{BP})$ extend down to very light isotopic values of $-27 \% \quad \delta^{13} \mathrm{C}$ and $<15 \%$ o $\delta^{18} \mathrm{O}$. Such light values have been attributed to kinetic fractionation effects resulting from rapid carbonate deposition in hyperalkaline pools (Clark et al., 1992; Wilson et al., 2010; Kelemen et al., 2011). Data from this study increase the upper bound of $\delta^{13} \mathrm{C}$ and $\delta^{18} \mathrm{O}$ values in young travertines. Some young travertines that are composed predominantly of aragonite extend to values of $-12 \% \delta^{13} \mathrm{C}$ and $32 \% \delta^{18} \mathrm{O}$, suggesting different kinetic fractionation effects under the conditions of aragonite precipitation or, perhaps, biologically-mediated precipitation (McConnaughey, 1989a, b).

While showing considerable scatter, the older $(>1000 \mathrm{yr}$ BP) travertines are generally isotopically heavier than the young travertines. Both Clark et al. (1992) and Kelemen and Matter (2011) suggest diagenetic and/or re-crystallization processes (that occur under slower conditions in equilibrium with fresher wadi waters) to account for the heavier isotopic ratios in older samples. An alternative hypothesis proposed by Clark and 
Fontes (1990) and Clark et al. (1992) is that in the past during less arid conditions and hence higher water/rock ratios, the $\mathrm{pH}$ of the alkaline springs was lower, reducing the strength of kinetic fractionation processes. However, the new data from this study indicate that there are a few samples with ages from $\sim 39,000$ to $\sim 45,000$ yr BP that have isotopic values (most notably $\delta^{18} \mathrm{O}$, see Figure 14B) as light as the young travertines. These data indicate that the conditions leading to the extreme kinetic fractionation observed in modern travertine precipitates also occurred during periods in the past.

There are no obvious systematic relationships between age and $\delta^{13} \mathrm{C}$ and $\delta^{18} \mathrm{O}$ ratios for the Mg-rich carbonate veins (Figure 15). There are also no systematic differences between the outcrop veins and roadcut veins. The Mg-rich carbonate outcrop veins cover a broad range of $\delta^{13} \mathrm{C}(-10$ to $-0.2 \%$ VPDB $)$ and $\delta^{18} \mathrm{O}$ (20 to $41 \%$ VSMOW) values with no obvious temporal trends. The ${ }^{14} \mathrm{C}$ dead roadcut veins cover a broad range of both $\delta^{13} \mathrm{C}\left(-12\right.$ to $-2 \%$ VPDB) and $\delta^{18} \mathrm{O}$ (23 to $37 \%$ VSMOW) whereas the roadcut veins $<50,000$ yr BP have a similar range of $\delta^{13} \mathrm{C}(-8$ to $-3 \%$ VPDB $)$ but a much narrower range of $\delta^{18} \mathrm{O}$ (32 to $\left.33 \% \mathrm{VSMOW}\right)$. This may indicate that during the last 50,000 years, the fluids from which the carbonate veins deposited had similar isotopic characteristics, and the conditions of precipitation were similar. Since the ages of the ${ }^{14} \mathrm{C}$ dead samples are unknown, they may represent carbonates formed over a much longer period of time under different conditions and from fluids of different origins.

\subsection{Carbonate Formation Temperatures:}

Most of the hand-picked carbonate samples are composed primarily of a single carbonate mineral, so the $\delta^{18} \mathrm{O}$ value of the whole rock represents a close approximation 
of the $\delta^{18} \mathrm{O}$ value for the dominant mineral. For these samples, approximate temperatures of formation can be estimated by assuming that the carbonates formed in equilibrium with typical Oman groundwaters and alkaline spring waters, which have $\delta^{18} \mathrm{O}$ values ranging from $-2 \%$ to $1 \%$ (Neal and Stanger, 1985; Clark et al., 1992; Matter et al., 2006; Matter, Kelemen, et al., unpublished data). Formation temperatures can be calculated for the minerals calcite, dolomite, and magnesite using equilibrium exchange formulas in O’Neil et al. (1969), Friedman and O’Neil (1977), and Chacko and Deines (2008) after corrections of magnesite and dolomite isotopic measurements for fractionation during dissolution in phosphoric acid at $77^{\circ} \mathrm{C}$ using the equations given in Das Sharma et al. (2002). Calculated equilibrium formation temperatures are presented in Table 3.

The dolomite (all veins except for one channel-filling travertine) and magnesite (all veins) samples suggest relatively low temperatures of formation. The dolomites have equilibrium formation temperatures ranging from approximately $20^{\circ}-60^{\circ} \mathrm{C}$ while the magnesite samples fall within a tighter range of approximately $40^{\circ}-60^{\circ} \mathrm{C}$. There is no significant difference in the temperatures of formation between outcrop and roadcut veins or between ${ }^{14} \mathrm{C}$ dead and younger veins. The relatively low formation temperatures of the dolomite and magnesite subsurface veins are consistent with the hypotheses of Kelemen and Matter (2008), Kelemen et al. (2011), and Streit et al. (2012, submitted) that these veins were formed in a shallow weathering horizon.

The calcite-rich travertines provide a large range of temperatures from unrealistically high values of $100-120^{\circ} \mathrm{C}$ (for recently-formed precipitates and also for a 
few older samples from Wadi Sudari Travertine) to unrealistically low values of $0^{\circ} \mathrm{C}$. Approximately $60 \%$ of the samples have formation temperatures ranging from $20-60^{\circ} \mathrm{C}$, which are consistent with the range of air temperatures in Oman. The anomalous formation temperatures may reflect the impact of kinetic fractionation on both $\mathrm{C}$ and $\mathrm{O}$ isotopes. For example, the recently-formed precipitates have experienced significant kinetic fractionation of both $\mathrm{C}$ and $\mathrm{O}$ isotopes. Other travertines may also be affected by kinetic fractionation or other non-equilibrium formation conditions and/or by diagenesis. Formation temperatures were also calculated for three calcite-rich travertine veins. Only one sample (OM10-13P-MG) provides a realistic formation temperature around $30^{\circ} \mathrm{C}$. The other two samples give anomalously low and unrealistic temperatures suggesting that, contrary to the conclusion of Clark and Fontes (1990), the travertine veins do not form in equilibrium with surface waters.

\subsection{Estimating Natural Rates of Carbonate Formation in the Peridotite}

\section{Layer of the Samail Ophiolite:}

\subsubsection{Travertine Deposition Rates:}

Neal and Stanger (1985) note the locations of approximately fifty hyperalkaline springs on their map, and Kelemen and Matter (2008) estimate that there are about 45 travertine deposits in the ophiolite based on detailed mapping in the southern third of the ophiolite. Using an estimate of travertine area in the Samail Ophiolite of $\sim 10^{7} \mathrm{~m}^{2}$ of Kelemen and Matter (2008) and the travertine deposition rate of $\sim 0.1-0.3 \mathrm{~mm} / \mathrm{yr}$ for travertine terraces with ages between 30,000 and 45,000 $\mathrm{yr} \mathrm{BP}, \sim 1,000-3,000 \mathrm{~m}^{3} / \mathrm{yr}^{\circ}$ of travertine was deposited in the Samail Ophiolite during that time, sequestering $1-3 \times 10^{6}$ 
$\mathrm{kg} \mathrm{CO} / \mathrm{yr}$ (also see Kelemen et al., 2011, Supplementary Tables 1 and 2 and associated text).

Today, travertine deposition does not occur simultaneously over the total area of exposed travertine. Rather, travertine deposition directly on the surface is limited to a few millimeters or centimeters per year within a few tens of meters of the hyperalkaline springs found at each travertine deposit. The alkaline springs that precipitate travertines also migrate over the surface of the travertine with time (e.g. Neal and Stanger, 1985; Kelemen and Matter, 2008). In addition, cross-cutting veins (e.g. Figure 4 in Kelemen and Matter, 2008) demonstrate that there is precipitation of travertine within travertine terraces, the rate of which probably varies with space and time. Therefore, at any given time, travertine is likely being precipitated over a fraction of the total travertine area.

In this study, we have demonstrated net accumulation of travertine at onedimensional rates of $\sim 0.1-0.3 \mathrm{~mm} / \mathrm{yr}$, averaged over time periods of $\sim 12,000-15,000$ years. Clearly, these rates were not sustained over the entire area of each travertine deposit. For example, constant deposition of travertine at these rates over 50,000 years would result in travertine deposits $5-15 \mathrm{~km}$ thick. However, Samail travertine deposits are generally only 1-2 m thick. The rate of travertine deposition has clearly varied over space and time and also-based on field observations - there has been significant erosion of travertine deposits. Thus, we concur with Kelemen and Matter (2008) that the ages of the travertines reflect a balance between slow deposition and slow erosion, which has removed most travertine samples older than 50,000 years. 


\subsection{2: Natural $\mathrm{CO}_{2}$ Sequestration in Mg-Rich Carbonate Veins:}

The discovery of a significant number of ${ }^{14} \mathrm{C}$ dead $\mathrm{Mg}$-rich veins at roadcuts allows us to place further constraints on the natural rate of $\mathrm{CO}_{2}$ uptake in the carbonate veins that was estimated by Kelemen and Matter (2008). That estimate was based on an average vein age of $26,000 \mathrm{yr} \mathrm{BP}$ (and a maximum vein age of 50,000 $\mathrm{yr} \mathrm{BP}$ ) and the assumption that the carbonate veins were primarily deposited in a shallow weathering zone $\sim 15 \mathrm{~km}$ thick. This resulted in an estimate of $\sim 10^{12} \mathrm{~kg}$ of $\mathrm{CO}_{2}$ stored in subsurface carbonate veins in the peridotite layer of the Samail Ophiolite, yielding a natural uptake rate of $\sim 4 \times 10^{7} \mathrm{~kg} \mathrm{CO}_{2} / \mathrm{yr}$. However, the discovery of a significant number of ${ }^{14} \mathrm{C}$ dead Mg-rich carbonate veins requires a lowering of this estimate.

Futhermore, the depth of the peridotite weathering zone containing the Mg-rich carbonate veins is uncertain. The weathering horizon is at least $\sim 5-10 \mathrm{~m}$ based on the heights of roadcuts exposing carbonate veins. Based on physical mass balance considerations that assume that weathering of the ophiolite keeps pace with erosion of the ophiolite and also chemical mass balance calculations that indicate that the travertine / subsurface vein ratio should be $\sim 10$, Kelemen and Matter (2008) argue that the thickness of the weathering zone is $\sim 15 \mathrm{~m}$. However, previously Neal and Stanger (1985) suggested that the weathering zone could be much thicker based on geothermal heating of hyperalkaline spring waters. Neal and Stanger (1985) observed that hyperalkaline spring waters range in temperature from $21-41^{\circ} \mathrm{C}$, and that they are on average $4.3^{\circ} \mathrm{C}$ warmer than associated surface stream waters. Some hyperalkaline spring waters are up to $\sim 15^{\circ} \mathrm{C}$ warmer than associated surface stream waters. Assuming a geothermal gradient of 
$20^{\circ} \mathrm{C} / \mathrm{km}$ and isotropic thermal conditions, Neal and Stanger (1985) estimated that hyperalkaline waters may circulate as deep as $0.7 \mathrm{~km}$ in the peridotite layer of the ophiolite.

We have carried out a sensitivity analysis to examine the impact of the presence of ${ }^{14} \mathrm{C}$ dead subsurface veins and the depth of the weathering horizon on estimates of natural $\mathrm{CO}_{2}$ sequestration rates. Figure 16 illustrates how $\mathrm{CO}_{2}$ uptake rates vary as a function of the percentage $(0-50 \%)$ of ${ }^{14} \mathrm{C}$ dead veins and the depth of the weathering horizon. Figure 16 demonstrates that Mg-rich carbonate vein formation in the peridotite layer of the Samail Ophiolite consumes on the order of $10^{7} \mathrm{~kg} \mathrm{CO}_{2} / \mathrm{yr}$, which is order of magnitude consistent with the previous estimate by Kelemen and Matter (2008). It is notable that at shallow depths of weathering, the $\mathrm{CO}_{2}$ uptake rates are not very sensitive to the percentage of ${ }^{14} \mathrm{C}$ dead veins. Further refinement of estimates for the natural $\mathrm{CO}_{2}$ uptake rate will require both better constraints on the ages of veins, especially those older than 50,000 years, from a broadened set of sampling localities and improved estimates of the thickness of the peridotite weathering zone.

\section{Summary:}

New ${ }^{14} \mathrm{C}$ ages and stable $\mathrm{C}$ and $\mathrm{O}$ isotope analyses for carbonate alteration products in the mantle peridotite layer of the Samail Ophiolite, Sultanate of Oman, support the hypothesis (e.g. Kelemen and Matter, 2008) that these carbonates are primarily formed as a result of recent, low-temperature, near-surface alteration of the peridotite. Deposition of Ca-rich travertines from hyperalkaline springs is ongoing and has been occurring continuously over the past $\sim 50,000$ yr BP. Ca-rich carbonate veins 
associated with travertine deposits range in age from $\sim 4,000-36,000$ yr BP (average age: $15,000 \mathrm{yr}$ BP). Detailed subsampling of travertine terraces indicates that travertine deposition rates were $\sim 0.1-0.3 \mathrm{~mm} / \mathrm{yr}$ between 30,000 and $45,000 \mathrm{yr} \mathrm{BP}$. Using an estimate of total travertine area from Kelemen and Matter (2008), a maximum of $\sim 1,000$ $3,000 \mathrm{~m}^{3} / \mathrm{yr}$ of travertine was deposited in the Samail Ophiolite during this time period, sequestering 1-3 x $10^{6} \mathrm{~kg} \mathrm{CO}_{2} / \mathrm{yr}$ in surficial deposits. As noted by Kelemen and Matter (2008) and Kelemen et al. (2011), formation of these surface deposits was probably accompanied by subsurface formation of 5 to 15 times this amount in Ca-rich carbonate veins.

${ }^{14} \mathrm{C}$ ages of $\mathrm{Mg}$-rich carbonate veins exposed on the natural peridotite weathering surface indicate that these veins formed $\sim 8,000-45,000 \mathrm{yr}$ BP (average age: 35,000 yr BP). However, dating of less-weathered veins from three roadcuts has also demonstrated that there are a significant number of $\mathrm{Mg}$-rich veins which are older than 50,000 years. At Fanja Roadcut, $75 \%$ of dated veins are ${ }^{14} \mathrm{C}$ dead; however, a locality-weighted average suggests that more realistically approximately $40 \%$ of $\mathrm{Mg}$-rich veins sampled at roadcuts are ${ }^{14} \mathrm{C}$ dead. The new ${ }^{14} \mathrm{C}$ vein ages allow us to further constrain natural rates of $\mathrm{CO}_{2}$ sequestration in subsurface $\mathrm{Mg}$-rich carbonate veins to $\sim 10^{7} \mathrm{~kg} \mathrm{CO} / \mathrm{yr}$, in agreement with Kelemen and Matter (2008) and Kelemen et al. (2011) and depending on the proportion of ${ }^{14} \mathrm{C}$ dead veins and the thickness of the weathering zone in which the veins are forming. Further refinement of this carbonation rate will require a better understanding of the age distributions of $\mathrm{Mg}$-rich carbonate veins and also the thickness of the peridotite weathering zone. 


\section{Acknowledgments:}

This research was done in collaboration with my supervisors Kenneth Sims and Susan Humphris and my collaborator Peter Kelemen. Ali Al-Rajhi, Ministry of Commerce and Industry, Sultanate of Oman, and Sobhi Nasir, Sultan Qaboos University, are thanked for logistical support during field seasons. Jürg Matter, Everett Shock, Lisa Streit, Amelia Paukert, and Ernest Gauntlett, and others are thanked for assistance with Oman fieldwork and for scientific discussions. Jerzy Blustajn, Margaret Sulanowska, Lora Wingate, Karen Benedetto, Chris Weidman, Ann McNichol, Mark Roberts, and William Jenkins are thanked for mentorship in the laboratory and assistance with sample preparation and labwork. This research was funded by National Science Foundation grant EAR-1049281, the Deep Ocean Exploration Institute at Woods Hole Oceanographic Institution, the Academic Programs Office at Woods Hole Oceanographic Institution, the Mellon Foundation, and a Research Initiative in Science and Engineering Grant from Columbia University to Peter Kelemen and Jürg Matter and also Kelemen's Charles Francis Adams Chair at Columbia University. 
Table 1: Sample Locations and Descriptions

\begin{tabular}{|c|c|c|c|c|c|c|}
\hline Sample Name: & Location: & $\begin{array}{l}\text { UTM- } \\
\text { Easting: }\end{array}$ & $\begin{array}{c}\text { UTM- } \\
\text { Northing: }\end{array}$ & Description: & $\begin{array}{l}\text { Major Minerals } \\
\text { (XRD): }\end{array}$ & $\begin{array}{c}\text { Trace Minerals } \\
\text { (XRD): }\end{array}$ \\
\hline \multicolumn{7}{|l|}{ Travertines: } \\
\hline OM09-27C-MS & $\begin{array}{l}\text { Qafeefah } \\
\text { Travertine }\end{array}$ & 0646207 & 2533711 & Travertine terrace & calcite & $\begin{array}{c}\text { chrysotile, kaolinite, } \\
\text { other unidentified } \\
\text { clay }\end{array}$ \\
\hline OM09-31C-MS & $\begin{array}{l}\text { Qafeefah } \\
\text { Travertine }\end{array}$ & 0646222 & 2533888 & Travertine terrace & calcite & unidentified clay \\
\hline $\begin{array}{c}\text { OM09-32C-MS- } \\
\text { WHITE }\end{array}$ & $\begin{array}{l}\text { Qafeefah } \\
\text { Travertine }\end{array}$ & 0646145 & 2533666 & Travertine terrace & calcite & unidentified clay \\
\hline $\begin{array}{c}\text { OM09-32C-MS- } \\
\text { BROWN }\end{array}$ & $\begin{array}{l}\text { Qafeefah } \\
\text { Travertine }\end{array}$ & 0646145 & 2533666 & Travertine terrace & calcite, dolomite & $\begin{array}{c}\text { kaolinite, other } \\
\text { unidentified clay }\end{array}$ \\
\hline OM09-33C-MS & $\begin{array}{l}\text { Qafeefah } \\
\text { Travertine }\end{array}$ & 0646144 & 2533672 & Travertine vein & calcite & $\begin{array}{c}\text { dolomite, aragonite, } \\
\text { chrysotile, } \\
\text { unidentified clay } \\
\end{array}$ \\
\hline OM09-28C-MS & $\begin{array}{l}\text { Qafeefah } \\
\text { Travertine }\end{array}$ & 0646210 & 2533807 & Channel-filling travertine & calcite & unidentified clay \\
\hline OM09-6COPS-MS & $\begin{array}{l}\text { Qafeefah } \\
\text { Travertine }\end{array}$ & 0646107 & 2533645 & $\begin{array}{l}\text { Recently-formed travertine } \\
\text { precipitate }\end{array}$ & aragonite & $\begin{array}{c}\text { calcite, } \\
\text { hydromagnesite?, } \\
\text { spinel?, unidentified } \\
\text { clay }\end{array}$ \\
\hline OM09-7COPS-MS & $\begin{array}{l}\text { Qafeefah } \\
\text { Travertine }\end{array}$ & 0646107 & 2533645 & $\begin{array}{l}\text { Recently-formed travertine } \\
\text { precipitate }\end{array}$ & aragonite & $\begin{array}{c}\text { brucite, calcite, } \\
\text { spinel?, unidentified } \\
\text { clay }\end{array}$ \\
\hline OM10-1COPS-MG & $\begin{array}{l}\text { Qafeefah } \\
\text { Travertine }\end{array}$ & 0646117 & 2533648 & $\begin{array}{l}\text { Recently-formed travertine } \\
\text { precipitate }\end{array}$ & calcite & none \\
\hline OM10-2COPS-MG & $\begin{array}{l}\text { Qafeefah } \\
\text { Travertine }\end{array}$ & 0646072 & 2533678 & $\begin{array}{l}\text { Recently-formed travertine } \\
\text { precipitate }\end{array}$ & aragonite & $\begin{array}{c}\text { brucite, calcite, } \\
\text { unidentified clay }\end{array}$ \\
\hline $\begin{array}{l}\text { OM10-28C-MG- } \\
\text { WHITE }\end{array}$ & Misht Travertine & 0487358 & 2576102 & Travertine terrace & calcite & $\begin{array}{l}\text { dolomite, unidentified } \\
\text { clay }\end{array}$ \\
\hline $\begin{array}{l}\text { OM10-28C-MG- } \\
\text { BROWN }\end{array}$ & Misht Travertine & 0487358 & 2576102 & Travertine terrace & dolomite, calcite & $\begin{array}{c}\text { aragonite, } \\
\text { unidentified clay }\end{array}$ \\
\hline
\end{tabular}




\begin{tabular}{|c|c|c|c|c|c|c|}
\hline Sample Name: & Location: & $\begin{array}{c}\text { UTM- } \\
\text { Easting: }\end{array}$ & $\begin{array}{c}\text { UTM- } \\
\text { Northing: }\end{array}$ & Description: & $\begin{array}{l}\text { Major Minerals } \\
\text { (XRD): }\end{array}$ & $\begin{array}{c}\text { Trace Minerals } \\
\text { (XRD): }\end{array}$ \\
\hline OM10-29C-MG & Misht Travertine & 0487323 & 2576088 & Travertine terrace & calcite & $\begin{array}{l}\text { dolomite, unidentified } \\
\text { clay }\end{array}$ \\
\hline OM10-32C-MG-\#1 & Misht Travertine & 0487305 & 2576134 & $\begin{array}{l}\text { Travertine terrace, part of } \\
\text { sequence }\end{array}$ & calcite & none \\
\hline OM10-32C-MG-\#2 & Misht Travertine & 0487305 & 2576134 & $\begin{array}{c}\text { Travertine terrace, part of } \\
\text { sequence }\end{array}$ & calcite & smectite-kaolinite \\
\hline OM10-32C-MG-\#3 & Misht Travertine & 0487305 & 2576134 & $\begin{array}{c}\text { Travertine terrace, part of } \\
\text { sequence }\end{array}$ & calcite & unidentified clay \\
\hline OM10-32C-MG-\#4 & Misht Travertine & 0487305 & 2576134 & $\begin{array}{l}\text { Travertine terrace, part of } \\
\text { sequence }\end{array}$ & calcite & $\begin{array}{l}\text { kaolinite, other } \\
\text { unidentified clay }\end{array}$ \\
\hline OM10-32C-MG-\#5 & Misht Travertine & 0487305 & 2576134 & $\begin{array}{c}\text { Travertine terrace, part of } \\
\text { sequence }\end{array}$ & calcite & $\begin{array}{c}\text { kaolinite, other } \\
\text { unidentified clay }\end{array}$ \\
\hline OM10-32C-MG-\#6 & Misht Travertine & 0487305 & 2576134 & $\begin{array}{l}\text { Travertine terrace, part of } \\
\text { sequence }\end{array}$ & calcite & $\begin{array}{c}\text { dolomite, kaolinite, } \\
\text { other unidentified } \\
\text { clay }\end{array}$ \\
\hline OM10-32C-MG-\#7 & Misht Travertine & 0487306 & 2576134 & $\begin{array}{c}\text { Travertine terrace, part of } \\
\text { sequence }\end{array}$ & calcite & unidentified clay \\
\hline OM10-32C-MG-\#8 & Misht Travertine & 0487305 & 2576134 & $\begin{array}{l}\text { Travertine terrace, part of } \\
\text { sequence }\end{array}$ & calcite & none \\
\hline OM10-32C-MG-\#9 & Misht Travertine & 0487305 & 2576134 & $\begin{array}{l}\text { Travertine terrace, part of } \\
\text { sequence }\end{array}$ & calcite & none \\
\hline $\begin{array}{c}\text { OM10-32C-MG- } \\
\# 10 \\
\end{array}$ & Misht Travertine & 0487305 & 2576134 & $\begin{array}{c}\text { Travertine terrace, part of } \\
\text { sequence }\end{array}$ & calcite & unidentified clay \\
\hline $\begin{array}{c}\text { OM10-32C-MG- } \\
\# 11 \\
\end{array}$ & Misht Travertine & 0487305 & 2576134 & $\begin{array}{c}\text { Travertine terrace, part of } \\
\text { sequence }\end{array}$ & calcite & unidentified clay \\
\hline OM10-31C-MG & Misht Travertine & 0487305 & 2576134 & $\begin{array}{c}\text { Travertine terrace, part of } \\
\text { sequence } w / 32 \mathrm{C}\end{array}$ & calcite & unidentified clay \\
\hline OM10-33C-MG & Misht Travertine & 0487305 & 2576134 & $\begin{array}{c}\text { Travertine terrace, part of } \\
\text { sequence w/ 32C }\end{array}$ & calcite & unidentified clay \\
\hline OM10-36C-MG & Misht Travertine & 0487322 & 2576050 & Travertine terrace & calcite & unidentified clay \\
\hline OM10-38C-MG & Misht Travertine & 0487316 & 2576132 & Travertine vein & calcite & $\begin{array}{l}\text { brucite (small } \\
\text { amount) }\end{array}$ \\
\hline OM10-13P-MG & Misht Travertine & 0487305 & 2576134 & Travertine vein & calcite & $\begin{array}{l}\text { hydromagnesite?, } \\
\text { unidentified clay }\end{array}$ \\
\hline
\end{tabular}




\begin{tabular}{|c|c|c|c|c|c|c|}
\hline Sample Name: & Location: & $\begin{array}{c}\text { UTM- } \\
\text { Easting: }\end{array}$ & $\begin{array}{c}\text { UTM- } \\
\text { Northing: } \\
\end{array}$ & Description: & $\begin{array}{l}\text { Major Minerals } \\
\text { (XRD): }\end{array}$ & $\begin{array}{l}\text { Trace Minerals } \\
\text { (XRD): }\end{array}$ \\
\hline OM10-37C-MG & Misht Travertine & 0487322 & 2576050 & Channel-filling travertine & dolomite & $\begin{array}{c}\text { chrysotile, } \\
\text { unidentified clay }\end{array}$ \\
\hline OM10-39C-MG & Misht Travertine & 0487316 & 2576132 & Channel-filling travertine & calcite & unidentified clay \\
\hline OM10-6COPS-MG & Misht Travertine & 0487316 & 2576132 & $\begin{array}{l}\text { Recently-formed travertine } \\
\text { precipitate }\end{array}$ & calcite & $\begin{array}{c}\text { aragonite? } \\
\text { unidentified clay }\end{array}$ \\
\hline OM09-88C-MS & $\begin{array}{c}\text { Wadi Sudari } \\
\text { Travertine }\end{array}$ & 0443088 & 2650306 & Terrace travertine & calcite & unidentified clay \\
\hline OM09-76C-MS-A & $\begin{array}{l}\text { Wadi Suadari } \\
\text { Travertine } \\
\end{array}$ & 0443115 & 2650257 & $\begin{array}{l}\text { Speleothem-like, layered } \\
\text { travertine (drop) }\end{array}$ & calcite & none \\
\hline OM09-76C-MS-B & $\begin{array}{l}\text { Wadi Suadari } \\
\text { Travertine }\end{array}$ & 0443115 & 2650257 & $\begin{array}{l}\text { Speleothem-like, layered } \\
\text { travertine (drop) }\end{array}$ & calcite & $\begin{array}{c}\text { unidentified clay } \\
\text { (very small amount) }\end{array}$ \\
\hline OM09-76C-MS-C & $\begin{array}{l}\text { Wadi Suadari } \\
\text { Travertine } \\
\end{array}$ & 0443115 & 2650257 & $\begin{array}{c}\text { Speleothem-like, layered } \\
\text { travertine (drop) }\end{array}$ & calcite & $\begin{array}{c}\text { unidentified clay } \\
\text { (very small amount) }\end{array}$ \\
\hline OM09-85C-MS & $\begin{array}{l}\text { Wadi Sudari } \\
\text { Travertine }\end{array}$ & 0443082 & 2650304 & $\begin{array}{l}\text { Newly-formed, stalagmite-like } \\
\text { travertine }\end{array}$ & calcite & unidentified clay \\
\hline OM09-84C-MS & $\begin{array}{l}\text { Wadi Sudari } \\
\text { Travertine } \\
\end{array}$ & 0443082 & 2650304 & Travertine vein & calcite, brucite & $\begin{array}{l}\text { hydromagnesite?, } \\
\text { unidentified clay }\end{array}$ \\
\hline OM09-87C-MS & $\begin{array}{l}\text { Wadi Sudari } \\
\text { Travertine } \\
\end{array}$ & 0443069 & 2650312 & Travertine vein & calcite & dolomite \\
\hline OM09-86C-MS & $\begin{array}{l}\text { Wadi Sudari } \\
\text { Travertine }\end{array}$ & 0443069 & 2650312 & Channel-filling travertine & calcite & $\begin{array}{l}\text { dolomite, unidentified } \\
\text { clay }\end{array}$ \\
\hline OM09-81C-MS & $\begin{array}{l}\text { Wadi Sudari } \\
\text { Travertine }\end{array}$ & 0443115 & 2650257 & Channel-filling travertine & calcite & unidentified clay \\
\hline OM09-89C-MS & $\begin{array}{l}\text { Wadi Sudari } \\
\text { Travertine }\end{array}$ & 0443118 & 2650078 & Channel-filling travertine & calcite & aragonite, brucite \\
\hline OM09-8COPS-MS & $\begin{array}{l}\text { Wadi Sudari } \\
\text { Travertine } \\
\end{array}$ & 0443118 & 2650078 & $\begin{array}{l}\text { Recently-formed travertine } \\
\text { precipitate }\end{array}$ & calcite & $\begin{array}{c}\text { aragonite, } \\
\text { hydromagnesite?, } \\
\text { unidentified clay }\end{array}$ \\
\hline OM09-106C-MS-A & $\begin{array}{c}\text { Wadi Uqaybah } \\
\text { Travertine }\end{array}$ & 0426245 & 2633924 & Terrace travertine & calcite & none \\
\hline OM09-106C-MS-B & $\begin{array}{l}\text { Wadi Uqaybah } \\
\text { Travertine }\end{array}$ & 0426245 & 2633924 & Terrace travertine & calcite & unidentified clay \\
\hline OM10-78C-MG-\#1 & $\begin{array}{c}\text { Wadi Uqaybah } \\
\text { Travertine }\end{array}$ & 0426266 & 2633919 & $\begin{array}{l}\text { Terrace travertine, part of } \\
\text { sequence }\end{array}$ & halite & $\begin{array}{l}\text { calcite, dolomite, } \\
\text { unidentified clay }\end{array}$ \\
\hline
\end{tabular}




\begin{tabular}{|c|c|c|c|c|c|c|}
\hline Sample Name: & Location: & $\begin{array}{l}\text { UTM- } \\
\text { Easting: }\end{array}$ & $\begin{array}{c}\text { UTM- } \\
\text { Northing: }\end{array}$ & Description: & $\begin{array}{l}\text { Major Minerals } \\
\text { (XRD): }\end{array}$ & $\begin{array}{c}\text { Trace Minerals } \\
\text { (XRD): }\end{array}$ \\
\hline OM10-78C-MG-\#2 & $\begin{array}{l}\text { Wadi Uqaybah } \\
\text { Travertine }\end{array}$ & 0426266 & 2633919 & $\begin{array}{l}\text { Terrace travertine, part of } \\
\text { sequence }\end{array}$ & halite & $\begin{array}{l}\text { dolomite, unidentified } \\
\text { clay }\end{array}$ \\
\hline OM10-78C-MG-\#3 & $\begin{array}{l}\text { Wadi Uqaybah } \\
\text { Travertine } \\
\end{array}$ & 0426266 & 2633919 & $\begin{array}{l}\text { Terrace travertine, part of } \\
\text { sequence }\end{array}$ & calcite & unidentified clay \\
\hline OM10-78C-MG-\#4 & $\begin{array}{c}\text { Wadi Uqaybah } \\
\text { Travertine }\end{array}$ & 0426266 & 2633919 & $\begin{array}{l}\text { Terrace travertine, part of } \\
\text { sequence }\end{array}$ & calcite & $\begin{array}{l}\text { dolomite, unidentified } \\
\text { clay }\end{array}$ \\
\hline OM10-78C-MG-\#5 & $\begin{array}{l}\text { Wadi Uqaybah } \\
\text { Travertine }\end{array}$ & 0426266 & 2633919 & $\begin{array}{c}\text { Terrace travertine, part of } \\
\text { sequence }\end{array}$ & calcite & unidentified clay \\
\hline OM10-78C-MG- \#6 & $\begin{array}{c}\text { Wadi Uqaybah } \\
\text { Travertine } \\
\end{array}$ & 0426266 & 2633919 & $\begin{array}{c}\text { Terrace travertine, part of } \\
\text { sequence }\end{array}$ & calcite & unidentified clay \\
\hline OM09-107C-MS & $\begin{array}{l}\text { Wadi Uqaybah } \\
\text { Travertine } \\
\end{array}$ & 0426309 & 2633950 & $\begin{array}{c}\text { Terrace travertine, part of } \\
\text { sequence }\end{array}$ & calcite & unidentified clay \\
\hline OM09-108C-MS & $\begin{array}{l}\text { Wadi Uqaybah } \\
\text { Travertine } \\
\end{array}$ & 0426215 & 2633925 & Travertine vein & dolomite & $\begin{array}{c}\text { monohydrocalcite?, } \\
\text { quartz? }\end{array}$ \\
\hline OM09-109C-MS & $\begin{array}{c}\text { Wadi Uqaybah } \\
\text { Travertine } \\
\end{array}$ & 0426208 & 2633925 & Channel-filling travertine & calcite & none \\
\hline $\begin{array}{c}\text { OM10-10COPS- } \\
\text { MS } \\
\end{array}$ & $\begin{array}{l}\text { Wadi Uqaybah } \\
\text { Travertine } \\
\end{array}$ & 0426183 & 2633965 & $\begin{array}{l}\text { Recently-formed travertine } \\
\text { precipitate } \\
\end{array}$ & calcite & $\begin{array}{l}\text { quartz?, unidentified } \\
\text { clay }\end{array}$ \\
\hline \multicolumn{7}{|l|}{$\begin{array}{l}\text { Mg-Rich Carbonate } \\
\text { Outcrop Veins: }\end{array}$} \\
\hline OM09-47C-MS & Fanja Roadcut & 0609304 & 2597565 & Carbonate outcrop vein & magnesite & unidentified clay \\
\hline OM10-13C-MG & Fanja Roadcut & 0609451 & 2597416 & Carbonate outcrop vein & magnesite & dolomite, calcite \\
\hline OM10-53C-MG & $\begin{array}{c}\text { Al-Wuqbah } \\
\text { Roadcut }\end{array}$ & 0440574 & 2643360 & Carbonate outcrop vein & magnesite & none \\
\hline OM09-91C-MS & $\begin{array}{l}\text { Wadi Sudari } \\
\text { Campsite }\end{array}$ & 0446151 & 2647471 & Carbonate outcrop vein & magnesite & calcite \\
\hline OM10-82C-MG & $\begin{array}{l}\text { Wadi Sudari } \\
\text { Campsite }\end{array}$ & 0445905 & 2647602 & Carbonate outcrop vein & magnesite & $\begin{array}{l}\text { dolomite, calcite, } \\
\text { unidentified clay }\end{array}$ \\
\hline OM10-83C-MG & $\begin{array}{l}\text { Wadi Sudari } \\
\text { Campsite }\end{array}$ & 0445905 & 2647602 & Carbonate outcrop vein & dolomite, calcite & $\begin{array}{c}\text { magnesite, } \\
\text { unidentified clay }\end{array}$ \\
\hline OM10-84C-MG & $\begin{array}{l}\text { Wadi Sudari } \\
\text { Campsite }\end{array}$ & 0445991 & 2647684 & Carbonate outcrop vein & brucite, calcite & $\begin{array}{l}\text { magnesite, dolomite, } \\
\text { unidentified clay }\end{array}$ \\
\hline
\end{tabular}




\begin{tabular}{|c|c|c|c|c|c|c|}
\hline Sample Name: & Location: & $\begin{array}{l}\text { UTM- } \\
\text { Easting: }\end{array}$ & $\begin{array}{c}\text { UTM- } \\
\text { Northing: }\end{array}$ & Description: & $\begin{array}{c}\text { Major Minerals } \\
\text { (XRD): }\end{array}$ & $\begin{array}{c}\text { Trace Minerals } \\
\text { (XRD): }\end{array}$ \\
\hline \multicolumn{7}{|l|}{$\begin{array}{l}\text { Mg-Rich Carbonate } \\
\text { Roadcut Veins: }\end{array}$} \\
\hline OM09-35C-MS & $\begin{array}{l}\text { Qafeefah } \\
\text { Roadcut }\end{array}$ & 0647852 & 2537682 & $\begin{array}{c}\text { Carbonate/serpentine roadcut } \\
\text { vein }\end{array}$ & magnesite & $\begin{array}{c}\text { chrysotile, } \\
\text { unidentified clay }\end{array}$ \\
\hline OM09-36C-MS & $\begin{array}{l}\text { Qafeefah } \\
\text { Roadcut } \\
\end{array}$ & 0647818 & 2537657 & Carbonate roadcut vein & magnesite & calcite, dolomite \\
\hline OM09-37C-MS & $\begin{array}{l}\text { Qafeefah } \\
\text { Roadcut }\end{array}$ & 0647821 & 2537649 & $\begin{array}{c}\text { Carbonate/serpentine roadcut } \\
\text { vein }\end{array}$ & dolomite & $\begin{array}{c}\text { magnesite, chrysotile, } \\
\text { calcite, brucite, } \\
\text { unidentified clay }\end{array}$ \\
\hline OM10-24C-MG & $\begin{array}{l}\text { Qafeefah } \\
\text { Roadcut }\end{array}$ & 0647791 & 2537644 & $\begin{array}{l}\text { Carbonate/serpentine roadcut } \\
\text { vein }\end{array}$ & magnesite & chrysotile, dolomite \\
\hline OM10-26C-MG & $\begin{array}{l}\text { Qafeefah } \\
\text { Roadcut }\end{array}$ & 0647791 & 2537644 & $\begin{array}{c}\text { Carbonate/serpentine roadcut } \\
\text { vein }\end{array}$ & magnesite & kaolinite, dolomite \\
\hline OM10-27C-MG & $\begin{array}{l}\text { Qafeefah } \\
\text { Roadcut }\end{array}$ & 0647791 & 2537644 & $\begin{array}{c}\text { Carbonate/serpentine roadcut } \\
\text { vein }\end{array}$ & magnesite & kaolinite, dolomite \\
\hline OM09-38C-MS & $\begin{array}{l}\text { Qafeefah } \\
\text { Roadcut }\end{array}$ & 0647782 & 2537641 & $\begin{array}{c}\text { Thin carbonate on roadcut } \\
\text { surface }\end{array}$ & magnesite & $\begin{array}{l}\text { dolomite, unidentified } \\
\text { clay }\end{array}$ \\
\hline OM10-25C-MG & $\begin{array}{l}\text { Qafeefah } \\
\text { Roadcut }\end{array}$ & 0647791 & 2537644 & $\begin{array}{c}\text { Thin carbonate on roadcut } \\
\text { surface }\end{array}$ & magnesite & dolomite, halite \\
\hline OM09-55C-MS & Fanja Roadcut & 0609351 & 2597507 & $\begin{array}{c}\text { Carbonate/serpentine roadcut } \\
\text { vein }\end{array}$ & dolomite & $\begin{array}{c}\text { aragonite?, } \\
\text { unidentified clay }\end{array}$ \\
\hline OM09-57C-MS & Fanja Roadcut & 0609356 & 2597501 & $\begin{array}{c}\text { Carbonate/serpentine roadcut } \\
\text { vein }\end{array}$ & dolomite & $\begin{array}{c}\text { aragonite?, calcite, } \\
\text { unidentified clay }\end{array}$ \\
\hline OM09-58C-MS & Fanja Roadcut & 0609370 & 2597506 & $\begin{array}{c}\text { Carbonate/serpentine roadcut } \\
\text { vein }\end{array}$ & dolomite, periclase? & $\begin{array}{c}\text { aragonite?, } \\
\text { unidentified clay }\end{array}$ \\
\hline OM09-60C-MS & Fanja Roadcut & 0609368 & 2597494 & $\begin{array}{c}\text { Carbonate/serpentine roadcut } \\
\text { vein }\end{array}$ & dolomite & $\begin{array}{c}\text { magnesite, kaolinite, } \\
\text { other unidentified } \\
\text { clay }\end{array}$ \\
\hline OM09-61C-MS & Fanja Roadcut & 0609379 & 2597499 & Carbonate roadcut vein & magnesite & dolomite \\
\hline OM09-62C-MS & Fanja Roadcut & 0609470 & 2597481 & Carbonate roadcut vein & magnesite & none \\
\hline OM09-63C-MS & Fanja Roadcut & 0609470 & 2597481 & Carbonate roadcut vein & magnesite & unidentified clay \\
\hline OM09-64C-MS & Fanja Roadcut & 0609470 & 2597481 & Carbonate roadcut vein & magnesite & dolomite \\
\hline
\end{tabular}




\begin{tabular}{|c|c|c|c|c|c|c|}
\hline Sample Name: & Location: & $\begin{array}{l}\text { UTM- } \\
\text { Easting: }\end{array}$ & $\begin{array}{c}\text { UTM- } \\
\text { Northing: }\end{array}$ & Description: & $\begin{array}{l}\text { Major Minerals } \\
\text { (XRD): }\end{array}$ & $\begin{array}{l}\text { Trace Minerals } \\
\text { (XRD): }\end{array}$ \\
\hline OM09-50C-MS & Fanja Roadcut & 0609279 & 2597526 & $\begin{array}{c}\text { Thin carbonate on roadcut } \\
\text { surface }\end{array}$ & dolomite, magnesite & unidentified clay \\
\hline OM10-48C-MG & $\begin{array}{l}\text { Al-Wuqbah } \\
\text { Roadcut }\end{array}$ & 0440574 & 2643360 & $\begin{array}{l}\text { Carbonate/serpentine roadcut } \\
\text { vein }\end{array}$ & dolomite & $\begin{array}{c}\text { chrysotile, kaolinite, } \\
\text { other unidentified } \\
\text { clay }\end{array}$ \\
\hline OM10-51C-MG & $\begin{array}{l}\text { Al-Wuqbah } \\
\text { Roadcut }\end{array}$ & 0440396 & 2643047 & Carbonate roadcut vein & $\begin{array}{c}\text { brucite, calcite, } \\
\text { chrysotile }\end{array}$ & $\begin{array}{c}\text { dolomite, kaolinite, } \\
\text { other unidentified } \\
\text { clay }\end{array}$ \\
\hline OM10-52C-MG & $\begin{array}{l}\text { Al-Wuqbah } \\
\text { Roadcut }\end{array}$ & 0440396 & 2643047 & Carbonate roadcut vein & magnesite & $\begin{array}{l}\text { halite?, unidentified } \\
\text { clay }\end{array}$ \\
\hline OM10-54C-MG & $\begin{array}{l}\text { Al-Wuqbah } \\
\text { Roadcut }\end{array}$ & 0440832 & 2643931 & $\begin{array}{l}\text { Carbonate/serpentine roadcut } \\
\text { vein }\end{array}$ & dolomite & $\begin{array}{c}\text { smectite-kaolinite, } \\
\text { other unidentified } \\
\text { clay }\end{array}$ \\
\hline $\begin{array}{l}\text { OM10-47C-MG- } \\
\text { CLEAR } \\
\end{array}$ & $\begin{array}{l}\text { Al-Wuqbah } \\
\text { Roadcut }\end{array}$ & 0440574 & 2643360 & $\begin{array}{c}\text { Thin carbonate on roadcut } \\
\text { surface } \\
\end{array}$ & dolomite & $\begin{array}{c}\text { aragonite?, } \\
\text { unidentified clay }\end{array}$ \\
\hline $\begin{array}{l}\text { OM10-47C-MG- } \\
\text { OPAQUE }\end{array}$ & $\begin{array}{l}\text { Al-Wuqbah } \\
\text { Roadcut }\end{array}$ & 0440574 & 2643360 & $\begin{array}{l}\text { Thin carbonate on roadcut } \\
\text { surface }\end{array}$ & dolomite & magnesite, calcite \\
\hline OM10-49C-MG & $\begin{array}{l}\text { Al-Wuqbah } \\
\text { Roadcut }\end{array}$ & 0440574 & 2643360 & $\begin{array}{l}\text { Thin carbonate on roadcut } \\
\text { surface }\end{array}$ & dolomite & $\begin{array}{c}\text { magnesite, } \\
\text { unidentified clay }\end{array}$ \\
\hline \multicolumn{7}{|l|}{$\begin{array}{c}\text { Kelemen et al., } \\
\text { Unpublished }\end{array}$} \\
\hline OM08-01 & $\begin{array}{c}\text { North of Village } \\
\text { of Falaij }\end{array}$ & 608957 & 2529931 & $\begin{array}{l}\text { shiny calcite veins from bottom } \\
\text { of falaj tunnel }\end{array}$ & -- & -- \\
\hline OM08-02A & $\begin{array}{c}\text { West } \\
\text { Makhibiyah } \\
\end{array}$ & 600173 & 2528469 & $\begin{array}{c}\text { blob of cement oozing from } \\
\text { carbonate-cementd } \\
\text { conglomerate }\end{array}$ & -- & -- \\
\hline OM08-200 & Kharma & 600090 & 2528421 & recently formed travertine & -- & -- \\
\hline OM08-202B & Kharma & 600173 & 2528469 & $\begin{array}{l}\mathrm{cm} \text { size perid gravel in carb } \\
\text { matrix } \\
\end{array}$ & -- & -- \\
\hline OM08-203-1 & Kharma & 600147 & 2528452 & $\begin{array}{c}\text { layered } \\
\text { conglomerate/travertine, should } \\
\text { be age progressive }\end{array}$ & -- & -- \\
\hline
\end{tabular}




\begin{tabular}{|c|c|c|c|c|c|c|}
\hline Sample Name: & Location: & $\begin{array}{c}\text { UTM- } \\
\text { Easting: }\end{array}$ & $\begin{array}{c}\text { UTM- } \\
\text { Northing: }\end{array}$ & Description: & $\begin{array}{l}\text { Major Minerals } \\
\text { (XRD): }\end{array}$ & $\begin{array}{c}\text { Trace Minerals } \\
\text { (XRD): }\end{array}$ \\
\hline OM08-203-2 & Kharma & 600147 & 2528452 & $\begin{array}{c}\text { layered } \\
\text { conglomerate/travertine, should } \\
\text { be age progressive }\end{array}$ & $x_{2}$ & 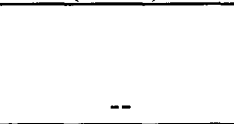 \\
\hline OM08-203 -3 & Kharma & 600147 & 2528452 & $\begin{array}{c}\text { layered } \\
\text { conglomerate/travertine, should } \\
\text { be age progressive }\end{array}$ & -- & -- \\
\hline OM08-203-4 & Kharma & 600147 & 2528452 & $\begin{array}{c}\text { layered } \\
\text { conglomerate/travertine, should } \\
\text { be age progressive }\end{array}$ & -- & -- \\
\hline OM08-210 & $\begin{array}{c}\text { Qafeefah-Dima } \\
\text { Pass } \\
\end{array}$ & 647967 & 2537823 & small veins in serp perid & -- & -- \\
\hline OM08-211 & $\begin{array}{c}\text { Qafeefah-Dima } \\
\text { Pass } \\
\end{array}$ & 647967 & 2537823 & thicker carb vein in serp perid & -- & -- \\
\hline OM08-215-1 & Wadi Dhuili & 667331 & 2542932 & veins in conglomerate & -- & -- \\
\hline OM08-215 -2 & Wadi Dhuili & 667331 & 2542932 & veins in conglomerate & -- & -- \\
\hline OM08-219A & Wadi Dhuili & 667340 & 2543071 & $\begin{array}{c}\text { carbonate-cemented } \\
\text { conglomerate }\end{array}$ & -- & -- \\
\hline OM08-219B & Wadi Dhuili & 667340 & 2543071 & $\begin{array}{c}\text { carbonate-cemented } \\
\text { conglomerate }\end{array}$ & -- & -- \\
\hline OM08-221-1 & $\begin{array}{l}\text { South of Batin } \\
\text { Camp }\end{array}$ & 671581 & 2535758 & $\begin{array}{c}\text { laminated, "micritic" vein in } \\
\text { serp harz }\end{array}$ & -- & -- \\
\hline OM08-221 - 2 & $\begin{array}{l}\text { South of Batin } \\
\text { Camp }\end{array}$ & 671581 & 2535758 & $\begin{array}{c}\text { laminated, "micritic" vein in } \\
\text { serp harz }\end{array}$ & -- & -- \\
\hline OM08-401 & Falaij & 608601 & 2527050 & $\begin{array}{c}\text { carb cemented } \\
\text { conglomerate/travertine }\end{array}$ & -- & -- \\
\hline OM08-402 & Falaij & 608601 & 2527050 & veins below travertine & -- & -- \\
\hline OM08-404 & Falaij & 608403 & 2527059 & mid-level travertine terrace & -- & -- \\
\hline OM08-406 & Duck & 586349 & 2523218 & karsty upper travertine terrace & -- & -- \\
\hline OM08-408 & Duck & 586089 & 2523083 & $\begin{array}{c}\text { top of mid-level travertine } \\
\text { terrace }\end{array}$ & -- & -- \\
\hline OM08-409 & Duck & 585927 & 2522933 & $\begin{array}{l}\text { travertine from plane of current } \\
\text { spring drainage }\end{array}$ & -- & -- \\
\hline
\end{tabular}




\begin{tabular}{|c|c|c|c|c|c|c|}
\hline Sample Name: & Location: & $\begin{array}{c}\text { UTM- } \\
\text { Easting: }\end{array}$ & $\begin{array}{c}\text { UTM- } \\
\text { Northing: }\end{array}$ & Description: & $\begin{array}{c}\text { Major Minerals } \\
\text { (XRD): }\end{array}$ & $\begin{array}{c}\text { Trace Minerals } \\
\text { (XRD): }\end{array}$ \\
\hline OM08-414 & Falaij & 608551 & 2525874 & $\begin{array}{c}\text { top-level terrace in south part of } \\
\text { Falaij }\end{array}$ & -- & -- \\
\hline OM08-415 & Falaij & 608429 & 2525929 & $\begin{array}{c}\text { mid-level terrace in south part } \\
\text { of Falaij }\end{array}$ & -- & -- \\
\hline OM08-416 & Falaij & 608279 & 2525960 & $\begin{array}{c}\text { lower-level terrace in south part } \\
\text { of Falaij }\end{array}$ & -- & -- \\
\hline OM08-450 & Duck & 584622 & 2525018 & $\begin{array}{c}\text { chalky white carbonate vein up } \\
\text { wadi from travertine }\end{array}$ & -- & - \\
\hline OM08-452 & Duck & 584446 & 2524269 & top of travertine & -- & -- \\
\hline OM08-453 & Duck & 584446 & 2524269 & middle of travertine & -- & - \\
\hline
\end{tabular}

Major and trace mineral identifications were determined on powdered samples by X-ray Diffraction at Woods Hole Oceanographic Institution. The minerals were identified using MacDiff, Version 4.2.5. Kelemen et al. (unpublished) analyses for carbonates collected during a 2008 field season are given for reference. These 2008 samples were prepared using different sub-sampling and leaching protocols. For the sample names, OMX indicates the year (09 for 2009; 10 for 2010) that the samples were collected in Oman. The middle number is a sample identification number. Finally, the last two letters indicate the people who collected the samples. In 2009 M stands for Mervine (Evelyn Mervine), and S stands for Sims (Kenneth Sims). In 2010 the M stands again for Mervine, and G stands for Gauntlett (Ernest Gauntlett). 
Table 2: ${ }^{14} \mathrm{C}$ Data and Ages

\begin{tabular}{|c|c|c|c|c|c|c|c|}
\hline Sample Name: & $\begin{array}{l}{ }^{14} \mathrm{C} \text { Dating } \\
\text { Technique: }\end{array}$ & $\begin{array}{c}\text { Fraction } \\
\text { Modern }{ }^{14} \mathrm{C} \text { : }\end{array}$ & Error: & $\begin{array}{c}\text { Uncalibrated }^{14} \mathrm{C} \\
\text { Age (yr BP): }\end{array}$ & Error: & $\begin{array}{c}\text { Calibrated }{ }^{14} \mathrm{C} \\
\text { Age (yr BP): }\end{array}$ & Error: \\
\hline \multicolumn{8}{|l|}{ Travertines: } \\
\hline OM09-27C-MS & Standard Dissolution & 0.2797 & 0.0027 & 10250 & 75 & 11971 & 152 \\
\hline OM09-31C-MS & Standard Dissolution & 0.0968 & 0.0010 & 18750 & 80 & 22351 & 103 \\
\hline OM09-32C-MS-WHITE & Standard Dissolution & 0.0105 & 0.0006 & 36600 & 450 & 42299 & 337 \\
\hline $\begin{array}{c}\text { OM09-32C-MS- } \\
\text { BROWN } \\
\end{array}$ & Standard Dissolution & 0.0484 & 0.0008 & 24300 & 130 & 29158 & 249 \\
\hline OM09-33C-MS & Standard Dissolution & 0.5868 & 0.0023 & 4280 & 30 & 4848 & 11 \\
\hline OM09-28C-MS & Standard Dissolution & 0.5661 & 0.0026 & 4570 & 35 & 3351 & 19 \\
\hline OM09-6COPS-MS & Standard Dissolution & 0.9710 & 0.0034 & 235 & 30 & 294 & 12 \\
\hline OM09-7COPS-MS & Sealed Tube & 0.9569 & 0.0052 & 354 & 44 & 350 & 30 \\
\hline OM10-1COPS-MG & Sealed Tube & 1.0064 & 0.0047 & $>$ modern & -- & $>$ modern & -- \\
\hline \multirow[t]{3}{*}{ OM10-2COPS-MG } & Sealed Tube & 1.0017 & 0.0072 & $>$ modern & -- & $>$ modern & -- \\
\hline & & 1.0026 & 0.0054 & $>$ modern & - & $>$ modern & -- \\
\hline & & 1.0022 & 0.0063 & $>$ modern & -- & $>$ modern & -- \\
\hline OM10-28C-MG-WHITE & Sealed Tube & 0.0200 & 0.0012 & 31406 & 483 & 35685 & 359 \\
\hline $\begin{array}{c}\text { OM10-28C-MG- } \\
\text { BROWN }\end{array}$ & Sealed Tube & 0.4092 & 0.0026 & 7178 & 52 & 7987 & 41 \\
\hline OM10-29C-MG & Sealed Tube & 0.1601 & 0.0019 & 14715 & 94 & 17861 & 166 \\
\hline \multirow[t]{4}{*}{ OM10-32C-MG-\#1 } & Sealed Tube & 0.0080 & 0.0012 & 38325 & 1209 & 38325 & 1209 \\
\hline & & 0.0052 & 0.0012 & 42260 & 1830 & 43183 & 877 \\
\hline & & 0.0060 & 0.0013 & 41155 & 1714 & 45748 & 1631 \\
\hline & & 0.0064 & 0.0012 & 40580 & 1584 & 44689 & 1326 \\
\hline OM10-32C-MG-\#2 & Sealed Tube & 0.0088 & 0.0012 & 38015 & 1073 & 42544 & 1564 \\
\hline OM10-32C-MG-\#3 & Sealed Tube & 0.0065 & 0.0012 & 40464 & 1457 & 40464 & 1457 \\
\hline OM10-32C-MG-\#4 & Sealed Tube & 0.0076 & 0.0012 & 39157 & 1237 & 43393 & 885 \\
\hline OM10-32C-MG-\#5 & Sealed Tube & 0.0231 & 0.0013 & 30277 & 443 & 34887 & 381 \\
\hline
\end{tabular}




\begin{tabular}{|c|c|c|c|c|c|c|c|}
\hline Sample Name: & $\begin{array}{l}{ }^{14} \mathrm{C} \text { Dating } \\
\text { Technique: }\end{array}$ & $\begin{array}{c}\text { Fraction } \\
\text { Modern }{ }^{14} \mathrm{C}:\end{array}$ & Error: & $\begin{array}{c}\text { Uncalibrated }^{14} \mathrm{C} \\
\text { Age (yr BP): }\end{array}$ & Error: & $\begin{array}{c}\text { Calibrated }^{14} \mathrm{C} \\
\text { Age (yr BP): } \\
\end{array}$ & Error: \\
\hline OM10-32C-MG-\#6 & Sealed Tube & 0.0259 & 0.0012 & 29347 & 385 & 34044 & 476 \\
\hline OM10-32C-MG-\#7 & Sealed Tube & -- & -- & -- & -- & -- & -- \\
\hline OM10-32C-MG-\#8 & Sealed Tube & 0.1854 & 0.0022 & 13535 & 96 & 16710 & 133 \\
\hline OM10-32C-MG-\#9 & Sealed Tube & 0.0311 & 0.0013 & 27867 & 324 & 32012 & 448 \\
\hline OM10-32C-MG-\#10 & Sealed Tube & 0.0843 & 0.0016 & 19866 & 153 & 23708 & 215 \\
\hline OM10-32C-MG-\#11 & Sealed Tube & 0.3689 & 0.0022 & 8011 & 48 & 8891 & 31 \\
\hline OM10-31C-MG & Sealed Tube & 0.0490 & 0.0013 & 24231 & 221 & 29051 & 148 \\
\hline OM10-33C-MG & Sealed Tube & 0.0329 & 0.0013 & 27425 & 306 & 31564 & 277 \\
\hline OM10-36C-MG & Sealed Tube & 0.0378 & 0.0013 & 26317 & 277 & 30971 & 201 \\
\hline OM10-38C-MG & Sealed Tube & 0.2329 & 0.0020 & 11705 & 69 & 13547 & 102 \\
\hline OM10-13P-MG & Sealed Tube & 0.0838 & 0.0015 & 19915 & 141 & 23750 & 221 \\
\hline OM10-37C-MG & Sealed Tube & 0.7644 & 0.0042 & 2159 & 45 & 2144 & 36 \\
\hline OM10-39C-MG & Sealed Tube & 0.7227 & 0.0047 & 2609 & 53 & 2746 & 40 \\
\hline OM10-6COPS-MG & Sealed Tube & 1.0036 & 0.0052 & $>$ modern & -- & $>$ modern & -- \\
\hline \multirow[t]{3}{*}{ OM09-88C-MS } & Standard Dissolution & 0.0200 & 0.0005 & 31400 & 180 & 35692 & 266 \\
\hline & & 0.0302 & 0.0007 & 28100 & 200 & 32297 & 392 \\
\hline & & 0.0251 & 0.0006 & 29750 & 190 & 33995 & 329 \\
\hline OM09-76C-MS-A & Standard Dissolution & 0.0254 & 0.0005 & 29500 & 160 & 34289 & 302 \\
\hline OM09-76C-MS-B & Standard Dissolution & 0.0140 & 0.0004 & 34300 & 230 & 39166 & 352 \\
\hline OM09-76C-MS-C & Standard Dissolution & 0.0075 & 0.0003 & 39300 & 320 & 43455 & 336 \\
\hline \multirow[t]{3}{*}{ OM09-85C-MS } & Standard Dissolution & 1.5496 & 0.0048 & $>$ modern & -- & $>$ modern & - \\
\hline & & 1.4632 & 0.0064 & $>$ modern & -- & $>$ modern & -- \\
\hline & & 1.5064 & 0.0056 & $>$ modern & - & $>$ modern & -- \\
\hline \multirow[t]{3}{*}{ OM09-84C-MS } & Standard Dissolution & 0.1294 & 0.0014 & 16450 & 90 & 19512 & 145 \\
\hline & & 0.1513 & 0.0014 & 15150 & 70 & 18200 & 175 \\
\hline & & 0.1403 & 0.0014 & 15800 & 80 & 18856 & 160 \\
\hline OM09-87C-MS & Standard Dissolution & 0.0824 & 0.0008 & 20100 & 80 & 24032 & 165 \\
\hline \multirow[t]{3}{*}{ OM09-86C-MS } & Standard Dissolution & 0.4601 & 0.0019 & 6240 & 35 & 7204 & 48 \\
\hline & & 0.3759 & 0.0021 & 7860 & 45 & 8655 & 65 \\
\hline & & 0.4180 & 0.0020 & 7050 & 40 & 7929 & 56 \\
\hline
\end{tabular}




\begin{tabular}{|c|c|c|c|c|c|c|c|}
\hline Sample Name: & $\begin{array}{c}{ }^{14} \mathrm{C} \text { Dating } \\
\text { Technique: }\end{array}$ & $\begin{array}{c}\text { Fraction } \\
\text { Modern }{ }^{14} \mathrm{C}:\end{array}$ & Error: & $\begin{array}{c}\text { Uncalibrated }{ }^{14} \mathrm{C} \\
\text { Age (yr BP): }\end{array}$ & Error: & $\begin{array}{c}\text { Calibrated }^{14} \mathrm{C} \\
\text { Age (yr BP): } \\
\end{array}$ & Error: \\
\hline OM09-81C-MS & Standard Dissolution & 1.043 & 0.0036 & >modern & -- & >modern & -- \\
\hline OM09-89C-MS & Standard Dissolution & 1.003 & 0.0042 & > modern & -- & $>$ modern & -- \\
\hline OM09-8COPS-MS & Standard Dissolution & 1.014 & 0.0031 & $>$ modern & -- & $>$ modern & -- \\
\hline OM09-106C-MS-A & Standard Dissolution & 0.0433 & 0.0007 & 25200 & 130 & 30023 & 235 \\
\hline \multirow[t]{3}{*}{ OM09-106C-MS-B } & Standard Dissolution & 0.0212 & 0.0006 & 31000 & 230 & 35316 & 232 \\
\hline & & 0.0290 & 0.0007 & 28400 & 190 & 32772 & 366 \\
\hline & & 0.0251 & 0.0007 & 29700 & 210 & 34044 & 299 \\
\hline OM10-78C-MG-\#1 & -- & -- & -- & -- & -- & -- & -- \\
\hline OM10-78C-MG-\#2 & -- & -- & -- & -- & -- & -- & -- \\
\hline \multirow[t]{4}{*}{ OM10-78C-MG-\#3 } & Sealed Tube & 0.0114 & 0.0012 & 35920 & 841 & 41005 & 816 \\
\hline & & 0.0102 & 0.0012 & 36829 & 939 & 41745 & 731 \\
\hline & & 0.0096 & 0.0013 & 37322 & 1067 & 42074 & 793 \\
\hline & & 0.0104 & 0.0012 & 36690 & 949 & 41608 & 780 \\
\hline OM10-78C-MG-\#4 & Sealed Tube & 0.0138 & 0.0012 & 34427 & 688 & 39532 & 831 \\
\hline OM10-78C-MG-\#5 & Sealed Tube & 0.0089 & 0.0012 & 37949 & 1062 & 42499 & 772 \\
\hline OM10-78C-MG-\#6 & Sealed Tube & 0.0170 & 0.0019 & 32727 & 894 & 37544 & 995 \\
\hline OM09-107C-MS & Standard Dissolution & 0.0270 & 0.0005 & 29000 & 150 & 33599 & 358 \\
\hline OM09-108C-MS & Standard Dissolution & 0.3659 & 0.0020 & 8070 & 45 & 9005 & 29 \\
\hline \multirow[t]{3}{*}{ OM09-109C-MS } & Standard Dissolution & 0.5326 & 0.0026 & 5060 & 40 & 5867 & 25 \\
\hline & & 0.5536 & 0.0024 & 4750 & 35 & 5543 & 78 \\
\hline & & 0.5431 & 0.0025 & 4905 & 38 & 5705 & 52 \\
\hline OM09-10COPS-MS & Standard Dissolution & 1.0046 & 0.0035 & >modern & -- & $>$ modern & -- \\
\hline \multicolumn{8}{|l|}{$\begin{array}{l}\text { Mg-Rich Carbonate } \\
\text { Outcrop Veins: }\end{array}$} \\
\hline \multirow[t]{3}{*}{ OM09-47C-MS } & Standard Dissolution & 0.0517 & 0.0009 & 23800 & 140 & 28559 & 239 \\
\hline & & 0.0510 & 0.0006 & 23900 & 90 & 28660 & 210 \\
\hline & & 0.0514 & 0.0008 & 23850 & 115 & 28610 & 225 \\
\hline OM10-13C-MG & Sealed Tube & 0.0093 & 0.0012 & 37534 & 1012 & 42220 & 731 \\
\hline OM10-53C-MG & Sealed Tube & 0.0091 & 0.0012 & 37783 & 1036 & 42387 & 747 \\
\hline
\end{tabular}




\begin{tabular}{|c|c|c|c|c|c|c|c|}
\hline Sample Name: & $\begin{array}{l}{ }^{14} \mathrm{C} \text { Dating } \\
\text { Technique: }\end{array}$ & $\begin{array}{c}\text { Fraction } \\
\text { Modern }{ }^{14} \mathrm{C}:\end{array}$ & Error: & $\begin{array}{c}\text { Uncalibrated }^{14} \mathrm{C} \\
\text { Age (yr BP): }\end{array}$ & Error: & $\begin{array}{c}\text { Calibrated }{ }^{14} \mathrm{C} \\
\text { Age (yr BP): }\end{array}$ & Error: \\
\hline & & 0.0072 & 0.0012 & 39628 & 1336 & 43688 & 948 \\
\hline & & 0.0079 & 0.0012 & 38918 & 1207 & 43246 & 873 \\
\hline & & 0.0080 & 0.0012 & 38776 & 1193 & 43107 & 856 \\
\hline OM09-91C-MS & Standard Dissolution & 0.0254 & 0.0006 & 29500 & 180 & 34277 & 320 \\
\hline OM10-82C-MG & Sealed Tube & 0.0158 & 0.0013 & 33318 & 639 & 37960 & 780 \\
\hline OM10-83C-MG & Sealed Tube & 0.0085 & 0.0012 & 38333 & 1124 & 42782 & 830 \\
\hline OM10-84C-MG & Sealed Tube & 0.0261 & 0.0012 & 29281 & 374 & 34007 & 481 \\
\hline \multicolumn{8}{|l|}{$\begin{array}{l}\text { Mg-Rich Carbonate } \\
\text { Roadcut Veins: }\end{array}$} \\
\hline OM09-35C-MS & Sealed Tube & 0.0057 & 0.0012 & 41547 & 1684 & 48496 & 1366 \\
\hline OM09-36C-MS & Sealed Tube & -0.0008 & 0.0012 & $>50000$ & -- & $>50000$ & -- \\
\hline OM09-37C-MS & Sealed Tube & 0.0036 & 0.0012 & 45275 & 2623 & $>50000$ & -- \\
\hline OM10-24C-MG & Sealed Tube & 0.0432 & 0.0015 & 25240 & 279 & 30007 & 312 \\
\hline OM10-26C-MG & Sealed Tube & 0.0995 & 0.0015 & 18535 & 118 & 22179 & 199 \\
\hline OM10-27C-MG & Sealed Tube & 0.0452 & 0.0017 & 24879 & 302 & 29697 & 202 \\
\hline OM09-38C-MS & Sealed Tube & 0.0181 & 0.0012 & 32214 & 552 & 38450 & 649 \\
\hline OM10-25C-MG & Sealed Tube & 0.0567 & 0.0014 & 23057 & 205 & 27922 & 286 \\
\hline \multirow[t]{3}{*}{ OM09-55C-MS } & Standard Dissolution & 0.0008 & 0.0002 & $>50000$ & -- & $>50000$ & -- \\
\hline & & 0.0004 & 0.0002 & $>50000$ & -- & $>50000$ & -- \\
\hline & & 0.0006 & 0.0002 & $>50000$ & - & $>50000$ & -- \\
\hline \multirow[t]{3}{*}{ OM09-57C-MS } & Standard Dissolution & 0.0010 & 0.0002 & $>50000$ & -- & $>50000$ & -- \\
\hline & & 0.0007 & 0.0002 & $>50000$ & -- & $>50000$ & -- \\
\hline & & 0.0009 & 0.0002 & $>50000$ & -- & $>50000$ & -- \\
\hline OM09-58C-MS & Standard Dissolution & 0.0015 & 0.0004 & $>50000$ & - & $>50000$ & -- \\
\hline OM09-60C-MS & Standard Dissolution & 0.0015 & 0.0002 & $>50000$ & -- & $>50000$ & - \\
\hline OM09-61C-MS & Standard Dissolution & 0.0032 & 0.0003 & 46100 & 660 & $>50000$ & - \\
\hline OM09-62C-MS & Standard Dissolution & 0.0016 & 0.0005 & $>50000$ & - & $>50000$ & - \\
\hline OM09-63C-MS & Standard Dissolution & 0.0030 & 0.0003 & 46600 & 860 & $>50000$ & -- \\
\hline OM09-64C-MS & Standard Dissolution & 0.0045 & 0.0004 & 43500 & 660 & 44497 & 651 \\
\hline
\end{tabular}




\begin{tabular}{|c|c|c|c|c|c|c|c|}
\hline Sample Name: & $\begin{array}{l}{ }^{14} \text { C Dating } \\
\text { Technique: }\end{array}$ & $\begin{array}{c}\text { Fraction } \\
\text { Modern }{ }^{14} \mathrm{C}:\end{array}$ & Error: & $\begin{array}{c}\text { Uncalibrated }^{14} \mathrm{C} \\
\text { Age (yr BP): }\end{array}$ & Error: & $\begin{array}{c}\text { Calibrated }{ }^{14} \mathrm{C} \\
\text { Age (yr BP): }\end{array}$ & Error: \\
\hline \multirow[t]{3}{*}{ OM09-50C-MS } & Standard Dissolution & 0.0364 & 0.0005 & 26600 & 120 & 31139 & 82 \\
\hline & & 0.0353 & 0.0006 & 26900 & 150 & 31256 & 93 \\
\hline & & 0.0358 & 0.0006 & 26750 & 135 & 31197 & 87 \\
\hline OM10-48C-MG & Sealed Tube & 0.0007 & 0.0012 & $>50000$ & -- & $>50000$ & -- \\
\hline OM10-51C-MG & Sealed Tube & 0.0805 & 0.0015 & 20240 & 150 & 24154 & 201 \\
\hline OM10-52C-MG & Sealed Tube & 0.0097 & 0.0012 & 37238 & 1013 & 43644 & 749 \\
\hline OM10-54C-MG & Sealed Tube & 0.0092 & 0.0012 & 37623 & 1076 & 42278 & 785 \\
\hline OM10-47C-MG-CLEAR & Sealed Tube & 0.0011 & 0.0012 & $>50000$ & -- & $>50000$ & -- \\
\hline $\begin{array}{c}\text { OM10-47C-MG- } \\
\text { OPAQUE } \\
\end{array}$ & Sealed Tube & 0.0293 & 0.0012 & 28365 & 341 & 32643 & 525 \\
\hline \multirow[t]{4}{*}{ OM10-49C-MG } & Sealed Tube & 0.0215 & 0.0012 & 30855 & 459 & 35234 & 347 \\
\hline & & 0.0156 & 0.0012 & 33420 & 611 & 38054 & 772 \\
\hline & & 0.0149 & 0.0013 & 33799 & 716 & 38497 & 1010 \\
\hline & & 0.0173 & 0.0012 & 32691 & 595 & 37261 & 709 \\
\hline \multicolumn{8}{|l|}{$\begin{array}{l}\text { Kelemen et al., } \\
\text { Unpublished }\end{array}$} \\
\hline OM08-01 & Standard Dissolution & 0.0020 & 0.0003 & $>50000$ & -- & $\mathrm{N} / \mathrm{A}$ & $\mathrm{N} / \mathrm{A}$ \\
\hline OM08-02A & Standard Dissolution & 0.0704 & 0.0009 & 21300 & 110 & 25461 & 210 \\
\hline OM08-200 & Standard Dissolution & 0.9681 & 0.0038 & 260 & 30 & 306 & 13 \\
\hline OM08-202B & Standard Dissolution & 0.0236 & 0.0004 & 30100 & 150 & 34783 & 123 \\
\hline OM08-203-1 & Standard Dissolution & 0.0079 & 0.0004 & 38900 & 400 & 43222 & 363 \\
\hline OM08-203-2 & Standard Dissolution & 0.0050 & 0.0003 & 42500 & 550 & 45720 & 449 \\
\hline OM08-203-3 & Standard Dissolution & 0.0149 & 0.0004 & 33800 & 210 & 38516 & 377 \\
\hline OM08-203-4 & Standard Dissolution & 0.0131 & 0.0004 & 34800 & 250 & 39817 & 415 \\
\hline OM08-210 & Standard Dissolution & 0.0030 & 0.0004 & $>50000$ & -- & N/A & $\mathrm{N} / \mathrm{A}$ \\
\hline OM08-211 & Standard Dissolution & 0.0106 & 0.0004 & 36500 & 270 & 41550 & 232 \\
\hline OM08-215-1 & Standard Dissolution & 0.6213 & 0.0026 & 3820 & 35 & 4221 & 40 \\
\hline OM08-215-2 & Standard Dissolution & 0.4121 & 0.0023 & 7120 & 45 & 7966 & 26 \\
\hline OM08-219A & Standard Dissolution & 0.1772 & 0.0014 & 13900 & 60 & 16981 & 100 \\
\hline
\end{tabular}




\begin{tabular}{|c|c|c|c|c|c|c|c|}
\hline Sample Name: & $\begin{array}{l}{ }^{14} \mathrm{C} \text { Dating } \\
\text { Technique: }\end{array}$ & $\begin{array}{c}\text { Fraction } \\
\text { Modern }{ }^{14} \mathrm{C}:\end{array}$ & Error: & $\begin{array}{c}\text { Uncalibrated }^{14} \mathrm{C} \\
\text { Age (yr BP): }\end{array}$ & Error: & $\begin{array}{c}\text { Calibrated }{ }^{14} \mathrm{C} \\
\text { Age (yr BP): }\end{array}$ & Error: \\
\hline OM08-219B & Standard Dissolution & 0.0784 & 0.0008 & 20500 & 80 & 24528 & 184 \\
\hline OM08-221 - 1 & Standard Dissolution & 0.0105 & 0.0003 & 36600 & 240 & 41614 & 216 \\
\hline OM08-221 - 2 & Standard Dissolution & 0.0083 & 0.0002 & 38500 & 230 & 42848 & 222 \\
\hline OM08-401 & Standard Dissolution & 0.0173 & 0.0004 & 32600 & 190 & 37193 & 313 \\
\hline OM08-402 & Standard Dissolution & 0.0232 & 0.0005 & 30200 & 170 & 34826 & 128 \\
\hline OM08-404 & Standard Dissolution & 0.0381 & 0.0005 & 26300 & 110 & 30970 & 115 \\
\hline OM08-406 & Standard Dissolution & 0.0223 & 0.0004 & 30500 & 130 & 34952 & 133 \\
\hline OM08-408 & Standard Dissolution & 0.0279 & 0.0005 & 28800 & 140 & 33465 & 349 \\
\hline OM08-409 & Standard Dissolution & 0.0151 & 0.0006 & 33700 & 310 & 38364 & 484 \\
\hline OM08-414 & Standard Dissolution & 0.0613 & 0.0007 & 22400 & 90 & 27160 & 294 \\
\hline OM08-415 & Standard Dissolution & 0.0499 & 0.0006 & 24100 & 100 & 28915 & 205 \\
\hline OM08-416 & Standard Dissolution & 0.0318 & 0.0005 & 27700 & 130 & 31855 & 225 \\
\hline OM08-450 & Standard Dissolution & 0.0350 & 0.0006 & 26900 & 130 & 31263 & 91 \\
\hline OM08-452 & Standard Dissolution & 0.0283 & 0.0005 & 28600 & 140 & 32925 & 285 \\
\hline OM08-453 & Standard Dissolution & 0.0090 & 0.0003 & 37800 & 240 & 42389 & 223 \\
\hline \multicolumn{8}{|l|}{$\begin{array}{l}\text { Repeat Analyses on } \\
\text { Kelemen and Matter } \\
\text { (2008) Samples: }\end{array}$} \\
\hline OM07-07 Original \#1 & Standard Dissolution & 0.0050 & 0.0002 & 42600 & 360 & 45740 & 324 \\
\hline OM07-07 Original \#2 & Standard Dissolution & 0.0020 & 0.0004 & 31300 & 160 & 35685 & 312 \\
\hline OM07-07 2009 Repeat & Standard Dissolution & 0.0347 & 0.0004 & 27000 & 90 & 31294 & 81 \\
\hline OM07-57 Original & Standard Dissolution & 0.0052 & 0.0002 & 42200 & 350 & 45503 & 621 \\
\hline OM07-57 2009 Repeat & Standard Dissolution & 0.0076 & 0.0003 & 39200 & 290 & 43357 & 313 \\
\hline
\end{tabular}

Replicate analyses are indicated in italics, with the average of replicate analyses given in bold. The calibrated ${ }^{14} \mathrm{C}$ ages were obtained using the Calib 6.0 Program with the IntCal09 calibration curve (Reimer et al., 2009). Kelemen et al. (unpublished) analyses for carbonates collected during a 2008 field season are given for reference. These were prepared using different subsampling and leaching protocols. The 2009 repeat analyses of Kelemen and Matter (2008) samples were carried out with the same preparation procedures, including more vigorous acid leaching in $10 \% \mathrm{HCl}$, as the other samples in this study. 
Table 3: Stable C and O Isotope Data

\begin{tabular}{|c|c|c|c|c|}
\hline Sample Name: & $\begin{array}{c}\delta^{13} \mathrm{C} \\
\text { VPDB: }\end{array}$ & $\begin{array}{c}\delta^{18} O \\
\text { VPDB: } \\
\end{array}$ & $\begin{array}{c}\delta^{18} O \\
\text { VSMOW: }\end{array}$ & $\begin{array}{c}\delta^{18} \mathrm{O} \text { Equilibrium } \\
\text { Temp. }\left({ }^{\circ} \mathrm{C}\right):\end{array}$ \\
\hline \multicolumn{5}{|l|}{ Travertines: } \\
\hline \multirow[t]{3}{*}{ OM09-27C-MS } & -5.63 & -1.39 & 29.43 & \\
\hline & -5.41 & -1.49 & 29.32 & \\
\hline & -5.52 & -1.44 & 29.37 & 11-19 \\
\hline OM09-31C-MS & -6.12 & -2.88 & 27.89 & $17-26$ \\
\hline \multirow[t]{3}{*}{ OM09-32C-MS-WHITE } & -3.05 & -4.99 & 25.72 & \\
\hline & -3.04 & -4.92 & 25.79 & \\
\hline & -3.04 & -4.95 & 25.75 & $26-36$ \\
\hline OM09-32C-MS-BROWN & -2.61 & -0.63 & 30.21 & -- \\
\hline OM09-33C-MS & 2.78 & 6.36 & 37.42 & $-6--10$ \\
\hline \multirow[t]{3}{*}{ OM09-28C-MS } & -10.98 & -6.54 & 24.12 & \\
\hline & -10.89 & -6.39 & 24.27 & \\
\hline & -10.93 & -6.47 & 24.19 & $34-45$ \\
\hline OM09-6COPS-MS & -15.96 & -2.96 & 27.81 & - \\
\hline OM09-7COPS-MS & -18.62 & -6.88 & 23.77 & -- \\
\hline \multirow[t]{3}{*}{ OM10-1COPS-MG } & -26.31 & -16.27 & 14.09 & \\
\hline & -26.30 & -16.33 & 14.03 & \\
\hline & -26.31 & -16.30 & 14.06 & $103-123$ \\
\hline \multirow[t]{3}{*}{ OM10-2COPS-MG } & -12.63 & 0.05 & 30.91 & \\
\hline & -12.64 & 0.07 & 30.93 & \\
\hline & -12.64 & 0.06 & 30.92 & -- \\
\hline \multirow[t]{3}{*}{ OM10-28C-MG-WHITE } & -10.49 & -2.93 & 27.84 & \\
\hline & -10.66 & -2.99 & 27.78 & \\
\hline & -10.58 & -2.96 & 27.81 & $17-26$ \\
\hline OM10-28C-MG-BROWN & 1.34 & 3.48 & 34.45 & -- \\
\hline OM10-29C-MG & -9.09 & -4.65 & 26.07 & $25-35$ \\
\hline \multirow[t]{3}{*}{ OM10-32C-MG-\#1 } & -17.88 & -7.79 & 22.83 & \\
\hline & -17.77 & -7.64 & 22.98 & \\
\hline & -17.82 & -7.71 & 22.91 & $40-52$ \\
\hline \multirow[t]{3}{*}{ OM10-32C-MG-\#2 } & -14.84 & -6.39 & 24.27 & \\
\hline & -14.80 & -6.42 & 24.24 & \\
\hline & -14.82 & -6.41 & 24.25 & $33-44$ \\
\hline \multirow[t]{4}{*}{ OM10-32C-MG-\#3 } & -13.53 & -11.53 & 18.98 & \\
\hline & -13.57 & -11.50 & 19.01 & \\
\hline & -13.66 & -11.52 & 18.99 & \\
\hline & -13.58 & -11.51 & 18.99 & $64-78$ \\
\hline \multirow[t]{3}{*}{ OM10-32C-MG-\#4 } & -18.19 & -10.19 & 20.35 & \\
\hline & -18.15 & -10.24 & 20.30 & \\
\hline & -18.17 & -10.22 & 20.33 & $55-69$ \\
\hline OM10-32C-MG-\#5 & -18.12 & -10.43 & 20.11 & \\
\hline
\end{tabular}




\begin{tabular}{|c|c|c|c|c|}
\hline Sample Name: & $\begin{array}{c}\delta^{13} \mathrm{C} \\
\text { VPDB: }\end{array}$ & $\begin{array}{c}\delta^{18} \mathrm{O} \\
\text { VPDB: }\end{array}$ & $\begin{array}{c}\delta^{18} \mathrm{O} \\
\text { VSMOW: }\end{array}$ & $\begin{array}{c}\delta^{18} \mathrm{O} \text { Equilibrium } \\
\text { Temp. }\left({ }^{\circ} \mathrm{C}\right):\end{array}$ \\
\hline & -18.08 & -10.49 & 20.05 & \\
\hline & -18.10 & -10.46 & 20.08 & $57-70$ \\
\hline \multirow[t]{3}{*}{ OM10-32C-MG-\#6 } & -20.56 & -10.47 & 20.06 & \\
\hline & -20.58 & -10.37 & 20.17 & \\
\hline & -20.57 & -10.42 & 20.12 & $56-70$ \\
\hline \multirow[t]{4}{*}{ OM10-32C-MG-\#7 } & -17.13 & -9.29 & 21.28 & \\
\hline & -17.38 & -9.26 & 21.32 & \\
\hline & -17.17 & -9.11 & 21.47 & \\
\hline & -17.23 & -9.22 & 21.36 & $56-60$ \\
\hline \multirow[t]{3}{*}{ OM10-32C-MG-\#8 } & -17.04 & -9.50 & 21.07 & \\
\hline & -17.05 & -9.51 & 21.05 & \\
\hline & -17.05 & -9.50 & 21.06 & $51-64$ \\
\hline \multirow[t]{3}{*}{ OM10-32C-MG- $\# 9$} & -18.19 & -10.26 & 20.28 & \\
\hline & -18.03 & -10.30 & 20.24 & \\
\hline & -18.11 & -10.28 & 20.26 & $56-69$ \\
\hline \multirow{3}{*}{ OM10-32C-MG-\#10 } & -11.21 & -3.77 & 26.97 & \\
\hline & -11.16 & -3.80 & 26.94 & \\
\hline & -11.19 & -3.79 & 26.95 & $21-30$ \\
\hline \multirow[t]{3}{*}{ OM10-32C-MG-\#11 } & -3.93 & 0.84 & 31.72 & \\
\hline & -3.82 & 0.74 & 31.62 & \\
\hline & -3.87 & 0.79 & 31.67 & $2-10$ \\
\hline \multirow[t]{3}{*}{ OM10-31C-MG } & -10.72 & -5.38 & 25.31 & \\
\hline & -10.79 & -4.91 & 25.80 & \\
\hline & -10.75 & -5.14 & 25.56 & $27-37$ \\
\hline OM10-33C-MG & -8.22 & -3.78 & 26.96 & $21-30$ \\
\hline OM10-36C-MG & -7.88 & -8.04 & 22.57 & $42-54$ \\
\hline \multirow{4}{*}{ OM10-38C-MG } & -7.99 & 1.04 & 31.93 & \\
\hline & -8.04 & 0.97 & 31.86 & \\
\hline & -8.04 & 1.14 & 32.03 & \\
\hline & -8.02 & 1.05 & 31.94 & $1-9$ \\
\hline OM10-13P-MG & -11.00 & -4.79 & 25.92 & $25-35$ \\
\hline OM10-37C-MG & 0.20 & 1.78 & 32.69 & $21-30$ \\
\hline OM10-39C-MG & -10.12 & -5.80 & 24.88 & $30-41$ \\
\hline \multirow[t]{3}{*}{ OM10-6COPS-MG } & -22.98 & -13.57 & 16.87 & \\
\hline & -22.88 & -13.64 & 16.80 & \\
\hline & -22.93 & -13.60 & 16.84 & $79-96$ \\
\hline OM09-88C-MS & -0.91 & -8.93 & 21.65 & $47-60$ \\
\hline \multirow[t]{3}{*}{ OM09-76C-MS-A } & -14.21 & -12.06 & 18.42 & \\
\hline & -14.37 & -12.10 & 18.38 & \\
\hline & -14.29 & -12.08 & 18.40 & $68-83$ \\
\hline OM09-76C-MS-B & -17.83 & -15.88 & 14.49 & $99-118$ \\
\hline OM09-76C-MS-C & -18.90 & -16.07 & 14.29 & $100-120$ \\
\hline OM09-85C-MS & -24.90 & -14.26 & 16.16 & $84-102$ \\
\hline OM09-84C-MS & -2.69 & -5.47 & 25.22 & -- \\
\hline
\end{tabular}




\begin{tabular}{|c|c|c|c|c|}
\hline Sample Name: & $\begin{array}{c}\delta^{13} \mathrm{C} \\
\text { VPDB: }\end{array}$ & $\begin{array}{c}\delta^{18} \mathrm{O} \\
\text { VPDB: }\end{array}$ & $\begin{array}{c}\delta^{18} O \\
\text { VSMOW: } \\
\end{array}$ & $\begin{array}{l}\delta^{18} \text { O Equilibrium } \\
\text { Temp. }\left({ }^{\circ} \mathrm{C}\right):\end{array}$ \\
\hline \multirow[t]{3}{*}{ OM09-87C-MS } & -8.76 & -4.91 & 25.80 & \\
\hline & -8.73 & -5.07 & 25.64 & \\
\hline & -8.75 & -4.99 & 25.72 & $26-36$ \\
\hline OM09-86C-MS & -8.61 & -9.72 & 20.84 & $52-65$ \\
\hline OM09-81C-MS & -26.66 & -15.73 & 14.64 & $97-117$ \\
\hline OM09-89C-MS & -22.23 & -12.52 & 17.95 & $71-86$ \\
\hline OM09-8COPS-MS & -20.04 & -8.74 & 21.85 & $46-58$ \\
\hline OM09-106C-MS-A & -10.44 & -4.97 & 25.74 & $23-33$ \\
\hline OM09-106C-MS-B & -14.14 & -8.87 & 21.72 & $47-59$ \\
\hline OM10-78C-MG-\#1 & -- & -- & -- & -- \\
\hline OM10-78C-MG-\#2 & -- & -- & -- & -- \\
\hline \multirow[t]{3}{*}{ OM10-78C-MG-\#3 } & -18.91 & -11.36 & 19.15 & \\
\hline & -18.93 & -11.38 & 19.13 & \\
\hline & -18.92 & -11.37 & 19.14 & $63-77$ \\
\hline \multirow[t]{3}{*}{ OM10-78C-MG-\#4 } & -20.24 & -13.02 & 17.44 & \\
\hline & -20.38 & -13.13 & 17.33 & \\
\hline & -20.31 & -13.07 & 17.38 & $75-91$ \\
\hline \multirow[t]{3}{*}{ OM10-78C-MG-\#5 } & -19.24 & -11.04 & 19.48 & \\
\hline & -19.14 & -10.93 & 19.60 & \\
\hline & -19.19 & -10.98 & $\mathbf{1 9 . 5 4}$ & $60-74$ \\
\hline \multirow[t]{3}{*}{ OM10-78C-MG-\#6 } & -5.16 & -3.69 & 27.06 & \\
\hline & -5.06 & -3.54 & 27.22 & \\
\hline & -5.11 & -3.61 & 27.14 & $20-29$ \\
\hline OM09-107C-MS & -12.03 & -6.42 & 24.24 & $33-44$ \\
\hline \multirow[t]{3}{*}{ OM09-108C-MS } & -2.81 & -0.30 & 30.55 & \\
\hline & -2.83 & -0.43 & 30.42 & \\
\hline & -2.82 & -0.36 & 30.49 & $21-35$ \\
\hline \multirow[t]{3}{*}{ OM09-109C-MS } & -11.17 & -4.57 & 26.14 & \\
\hline & -11.13 & -4.45 & 26.27 & \\
\hline & -11.15 & -4.51 & 26.21 & $24-34$ \\
\hline OM09-10COPS-MS & -23.23 & -14.60 & 15.81 & $87-105$ \\
\hline \multicolumn{5}{|c|}{$\begin{array}{l}\text { Mg-Rich Carbonate Outcrop } \\
\text { Veins: }\end{array}$} \\
\hline \multirow[t]{2}{*}{ OM09-47C-MS } & -3.81 & 4.69 & 35.70 & $41-49$ \\
\hline & & & & \\
\hline \multirow[t]{3}{*}{ OM10-13C-MG } & -2.25 & 3.36 & 34.33 & \\
\hline & -2.38 & 3.47 & 34.44 & \\
\hline & -2.31 & 3.42 & 34.38 & $46-55$ \\
\hline \multirow[t]{3}{*}{ OM10-53C-MG } & -0.79 & 3.58 & 34.55 & \\
\hline & -0.69 & 3.77 & 34.74 & \\
\hline & -0.74 & 3.67 & 34.64 & $45-54$ \\
\hline OM09-91C-MS & -3.45 & 2.44 & 33.37 & $50-60$ \\
\hline
\end{tabular}




\begin{tabular}{|c|c|c|c|c|}
\hline Sample Name: & $\begin{array}{c}\delta^{13} \mathrm{C} \\
\text { VPDB: }\end{array}$ & $\begin{array}{c}\delta^{18} \mathrm{O} \\
\text { VPDB: }\end{array}$ & $\begin{array}{c}\delta^{18} \mathrm{O} \\
\text { VSMOW: }\end{array}$ & $\begin{array}{l}\delta^{18} \mathrm{O} \text { Equilibrium } \\
\text { Temp. }\left({ }^{\circ} \mathrm{C}\right):\end{array}$ \\
\hline \multirow[t]{3}{*}{ OM10-82C-MG } & -5.95 & 2.49 & 33.42 & \\
\hline & -5.99 & 2.68 & 33.62 & \\
\hline & -5.97 & 2.58 & 33.52 & $50-59$ \\
\hline OM10-83C-MG & -10.41 & -3.72 & 27.03 & -- \\
\hline \multirow[t]{3}{*}{ OM10-84C-MG } & -2.49 & 5.34 & 36.37 & \\
\hline & -2.28 & 5.24 & 36.26 & \\
\hline & -2.38 & 5.29 & 36.32 & -- \\
\hline \multicolumn{5}{|c|}{$\begin{array}{l}\text { Mg-Rich Carbonate Roadcut } \\
\text { Veins: }\end{array}$} \\
\hline \multirow[t]{3}{*}{ OM09-35C-MS } & -7.19 & 2.64 & 33.58 & \\
\hline & -7.07 & 2.60 & 33.54 & \\
\hline & -7.13 & 2.62 & 33.56 & $50-59$ \\
\hline OM09-36C-MS & -6.32 & 0.82 & 31.70 & $58-68$ \\
\hline OM09-37C-MS & -2.84 & -0.50 & 30.34 & $52-62$ \\
\hline \multirow[t]{3}{*}{ OM10-24C-MG } & -6.42 & 2.04 & 32.96 & \\
\hline & -6.39 & 2.09 & 33.01 & \\
\hline & -6.41 & 2.06 & 32.99 & $52-62$ \\
\hline \multirow[t]{3}{*}{ OM10-26C-MG } & -7.29 & 1.96 & 32.88 & \\
\hline & -7.48 & 2.02 & 32.94 & \\
\hline & -7.38 & 1.99 & 32.91 & $53-62$ \\
\hline \multirow[t]{3}{*}{ OM10-27C-MG } & -7.05 & 2.35 & 33.29 & \\
\hline & -7.09 & 2.28 & 33.21 & \\
\hline & -7.07 & 2.32 & 33.25 & $51-61$ \\
\hline \multirow[t]{4}{*}{ OM09-38C-MS } & -7.99 & 1.04 & 31.93 & \\
\hline & -8.04 & 0.97 & 31.86 & \\
\hline & -8.04 & 1.14 & 32.03 & \\
\hline & -8.02 & 1.05 & 31.94 & $57-67$ \\
\hline \multirow[t]{3}{*}{ OM10-25C-MG } & -6.66 & 1.81 & 32.73 & \\
\hline & -6.72 & 1.78 & 32.69 & \\
\hline & -6.69 & 1.80 & 32.71 & $53-63$ \\
\hline \multirow[t]{3}{*}{ OM09-55C-MS } & -6.04 & -6.95 & 23.70 & \\
\hline & -6.07 & -7.04 & 23.60 & $64-77$ \\
\hline & -6.05 & -7.00 & 23.65 & \\
\hline OM09-57C-MS & -9.10 & -3.14 & 27.62 & $43-53$ \\
\hline OM09-58C-MS & -10.00 & -0.04 & 30.82 & $29-38$ \\
\hline OM09-60C-MS & -7.74 & 5.56 & 36.59 & $7-15$ \\
\hline OM09-61C-MS & -2.72 & 4.50 & 35.50 & $42-50$ \\
\hline OM09-62C-MS & -7.38 & 3.53 & 34.50 & $46-55$ \\
\hline OM09-63C-MS & -8.15 & 4.00 & 34.98 & $44-53$ \\
\hline \multirow[t]{3}{*}{ OM09-64C-MS } & -7.03 & 1.76 & 32.67 & \\
\hline & -7.38 & 2.20 & 33.13 & \\
\hline & -7.21 & 1.98 & 32.90 & $53-62$ \\
\hline OM09-50C-MS & -1.98 & 3.18 & 34.14 & -- \\
\hline
\end{tabular}




\begin{tabular}{|c|c|c|c|c|}
\hline Sample Name: & $\begin{array}{c}\delta^{13} \mathrm{C} \\
\text { VPDB: }\end{array}$ & $\begin{array}{c}\delta^{18} \mathrm{O} \\
\text { VPDB: } \\
\end{array}$ & $\begin{array}{c}\delta^{18} \mathrm{O} \\
\text { VSMOW: }\end{array}$ & $\begin{array}{c}\delta^{18} \mathrm{O} \text { Equilibrium } \\
\text { Temp. }\left({ }^{\circ} \mathrm{C}\right):\end{array}$ \\
\hline OM10-48C-MG & -7.57 & -0.72 & 30.12 & $33-43$ \\
\hline \multirow[t]{3}{*}{ OM10-51C-MG } & -12.63 & -4.50 & 26.22 & -- \\
\hline & -12.56 & -4.69 & 26.03 & \\
\hline & -12.59 & -4.59 & 26.13 & -- \\
\hline \multirow[t]{3}{*}{ OM10-52C-MG } & -7.72 & -0.03 & 30.83 & \\
\hline & -7.75 & 0.10 & 30.96 & \\
\hline & -7.74 & $\mathbf{0 . 0 3}$ & 30.90 & $62-73$ \\
\hline \multirow[t]{3}{*}{ OM10-54C-MG } & -7.37 & 2.48 & 33.42 & \\
\hline & -7.43 & 2.54 & 33.48 & \\
\hline & -7.40 & 2.51 & 33.45 & $19-27$ \\
\hline \multirow[t]{3}{*}{ OM10-47C-MG-CLEAR } & -7.04 & -3.86 & 26.88 & \\
\hline & -7.07 & -3.80 & 26.94 & \\
\hline & -7.05 & -3.83 & 26.91 & $47-57$ \\
\hline \multirow[t]{3}{*}{ OM10-47C-MG-OPAQUE } & -3.35 & 1.44 & 32.35 & \\
\hline & -3.50 & 1.43 & 32.34 & \\
\hline & -3.42 & 1.44 & 32.34 & $23-31$ \\
\hline \multirow[t]{3}{*}{ OM10-49C-MG } & -2.90 & 0.86 & 31.75 & \\
\hline & -2.81 & 0.96 & 31.85 & \\
\hline & -2.85 & 0.91 & 31.80 & $25-33$ \\
\hline
\end{tabular}

Replicate analyses are indicated in italics, with the average of replicate analyses given in bold. The approximate temperatures of formation were estimated by assuming that the carbonates formed in equilibrium with typical Oman groundwaters and alkaline spring waters, which have $\delta^{18} \mathrm{O}$ values ranging from $-2^{\circ} \%$ to $1 \%$ (Neal and Stanger, 1985; Clark et al., 1992; Matter et al., 2006; Matter, Kelemen, et al., unpublished data). The formation temperatures were then calculated for the minerals calcite, dolomite, and magnesite using equilibrium exchange formulas in O'Neil et al. (1969), Friedman and O'Neil (1977), and Chacko and Deines (2008) and also corrections for magnesite and dolomite isotope measurements for dissolution in phosphoric acid at $77^{\circ} \mathrm{C}$ given in Das Sharma et al. (2002). 
Table 4: Travertine Deposition Rates

\begin{tabular}{|c|c|c|c|}
\hline Type of Travertine: & Deposition Rate (mm / yr): & $\begin{array}{c}\text { Average Deposition } \\
\text { Rate (mm/yr): }\end{array}$ & Data Source: \\
\hline $\begin{array}{c}\text { Misht Travertine Sequence } \\
\begin{array}{c}\text { Wadi Uqaybah Travertine } \\
\text { Sequence \#1 }\end{array}\end{array}$ & 0.1 & -- & This Study \\
\hline $\begin{array}{c}\text { Wadi Uqaybah Travertine } \\
\text { Sequence \#2 }\end{array}$ & 0.3 & -- & This Study \\
\hline Wadi Sudari Pseudospeleothem & 0.3 & -- & This Study \\
\hline $\begin{array}{c}\text { Epigean Meteogene (All) } \\
\text { Cyanobacteria-Associated }\end{array}$ & 0.005 & -- & This Study \\
\hline $\begin{array}{c}\text { Eukaryotic Algae-Associated } \\
\text { Bryophyte-Associated }\end{array}$ & $0.04-48$ & $5.28(\mathrm{~N}=55)$ & $\begin{array}{c}\text { Pentecost } \\
(2005)\end{array}$ \\
\hline Speleothem & $0.04-7.0$ & $2.38(\mathrm{~N}=16)$ & $\begin{array}{c}\text { Pentecost } \\
(2005)\end{array}$ \\
\hline Thermogene & $0.42-48$ & $9.08(\mathrm{~N}=5)$ & $\begin{array}{c}\text { Pentecost } \\
(2005)\end{array}$ \\
\hline
\end{tabular}


Figures:

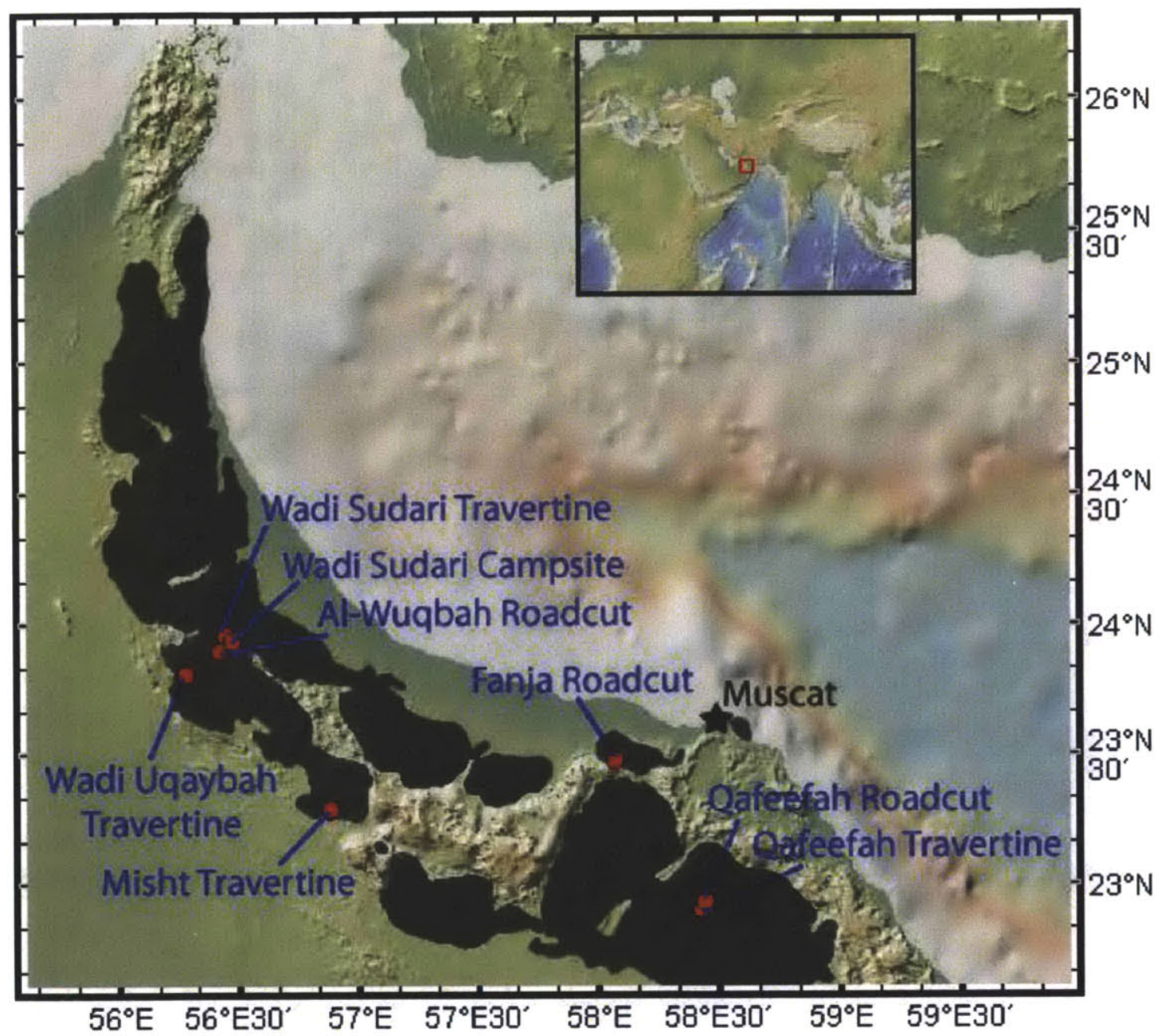

Figure 1: The eight field locations where carbonate and peridotite samples were collected during 2009 and 2010 field seasons. The Samail Ophiolite is outlined in dark gray, and sampling locations are indicated by red circles. Base map from GeoMapApp. 


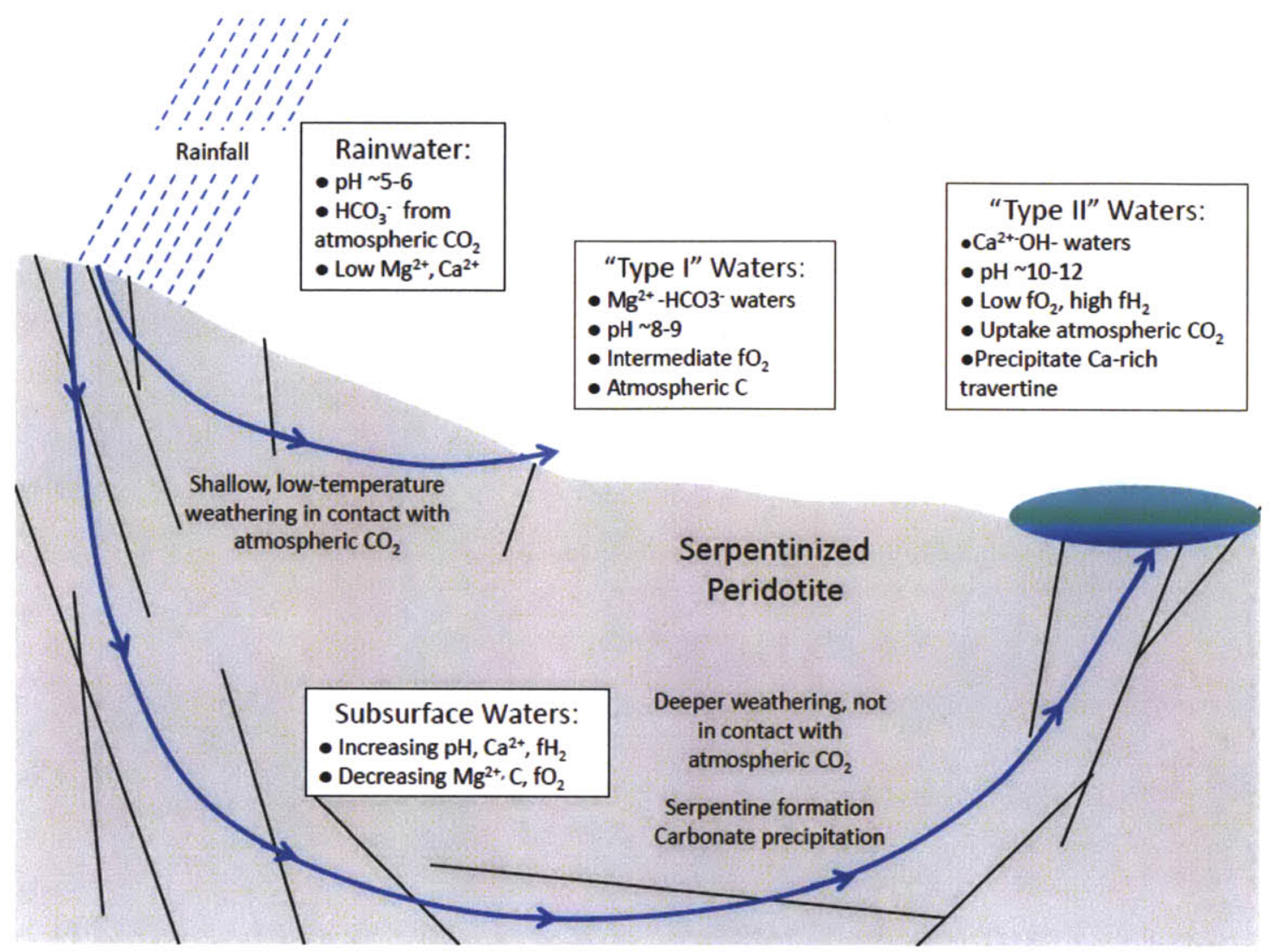

Figure 2: Schematic illustrating the behavior of $\mathrm{CO}_{2}$ in Type I and Type II waters associated with alteration of partially-serpentinized peridotite. Figure modified from Neal and Stanger (1985). 


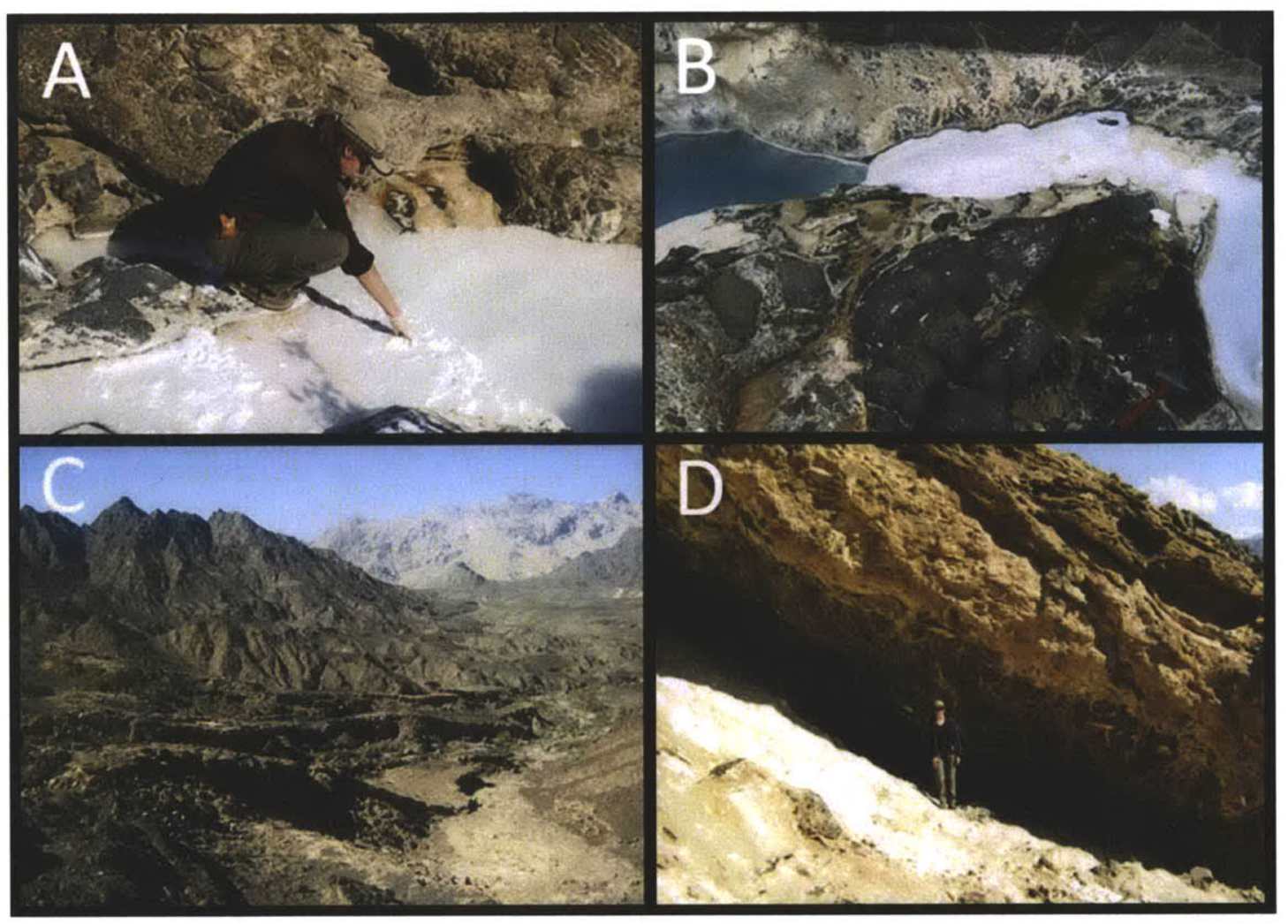

Figure 3: Representative images of calcite-rich travertines forming in the peridotite layer of the Samail Ophiolite. A: Recently-formed carbonate precipitate in a hyperalkaline streambed at Qafeefah Travertine. B: A turquoise alkaline pool and recently-formed carbonate precipitate at Wadi Sudari Travertine. Note the hammer for scale. C: Brown, fossil travertine terraces at Wadi Uqaybah Travertine. The travertine terraces are $\sim 1-2 \mathrm{~m}$ thick and are found extensively throughout the wadi. Note how the terraces are broken by incised channels, which are filled with lighter-colored travertines. Many of the incised channels also contain hyperalkaline pools and seeps. D: Calcite-rich travertine veins exposed in peridotite underneath a thick fossil travertine deposit at Wadi Sudari Travertine. 


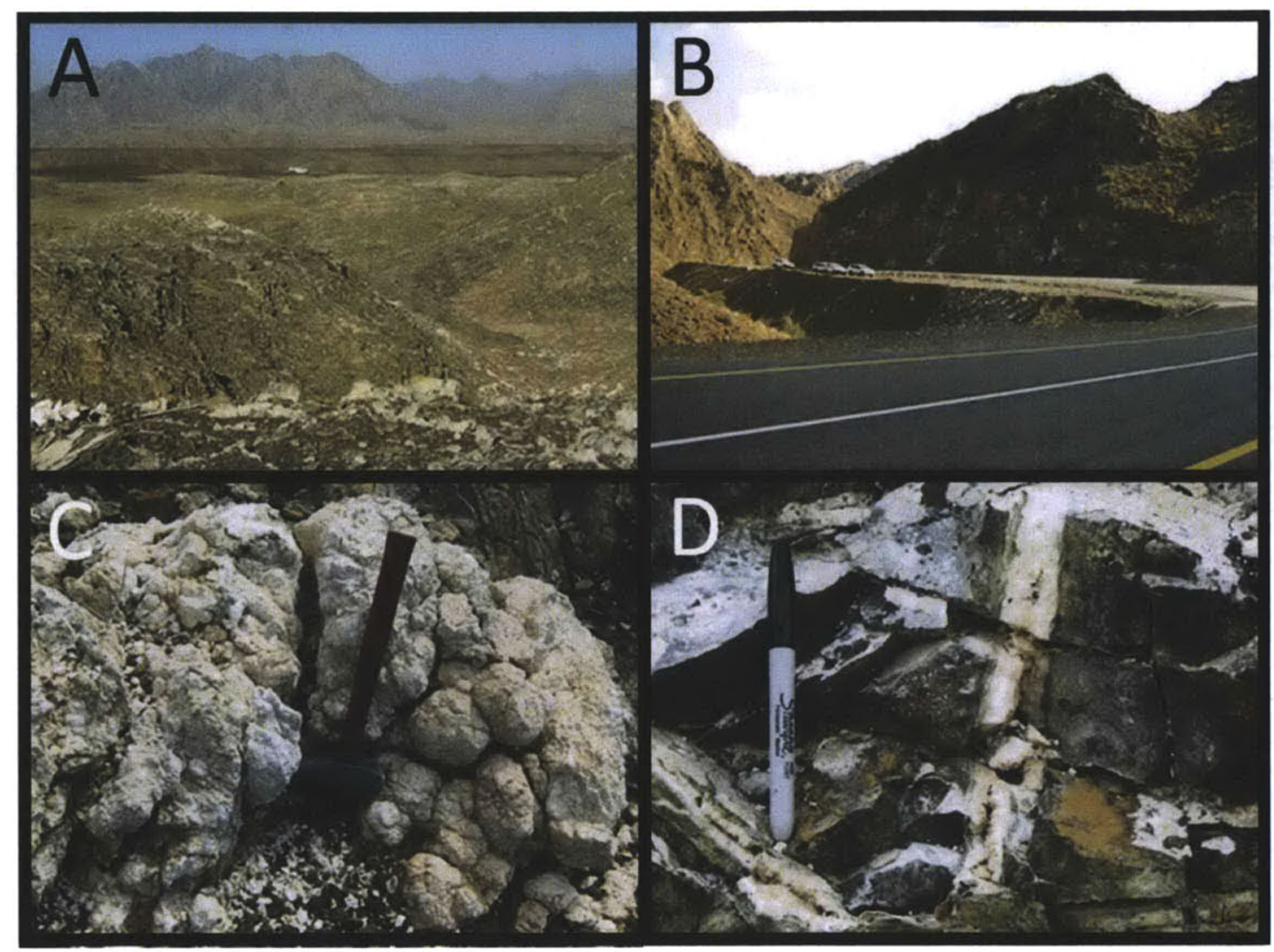

Figure 4: Representative images of $\mathrm{Mg}$-rich carbonate veins exposed on the natural peridotite weathering surface (A and $C$ ) and at roadcuts (B and D) in the Samail Ophiolite. A: Bright white magnesite veins exposed in outcrop on the natural peridotite weathering surface at Wadi Sudari Campsite. B: Magnesite, dolomite, and gabbro veins exposed on a roadcut through peridotite near the town of Fanja. Note the Land Cruisers for scale. C: A white magnesite vein with botryoidal texture exposed on the natural peridotite weathering surface near to Fanja Roadcut. Note the hammer for scale. D: Dolomite-serpentine veins exposed on the surface of Fanja Roadcut. Note the Sharpie marker for scale. 


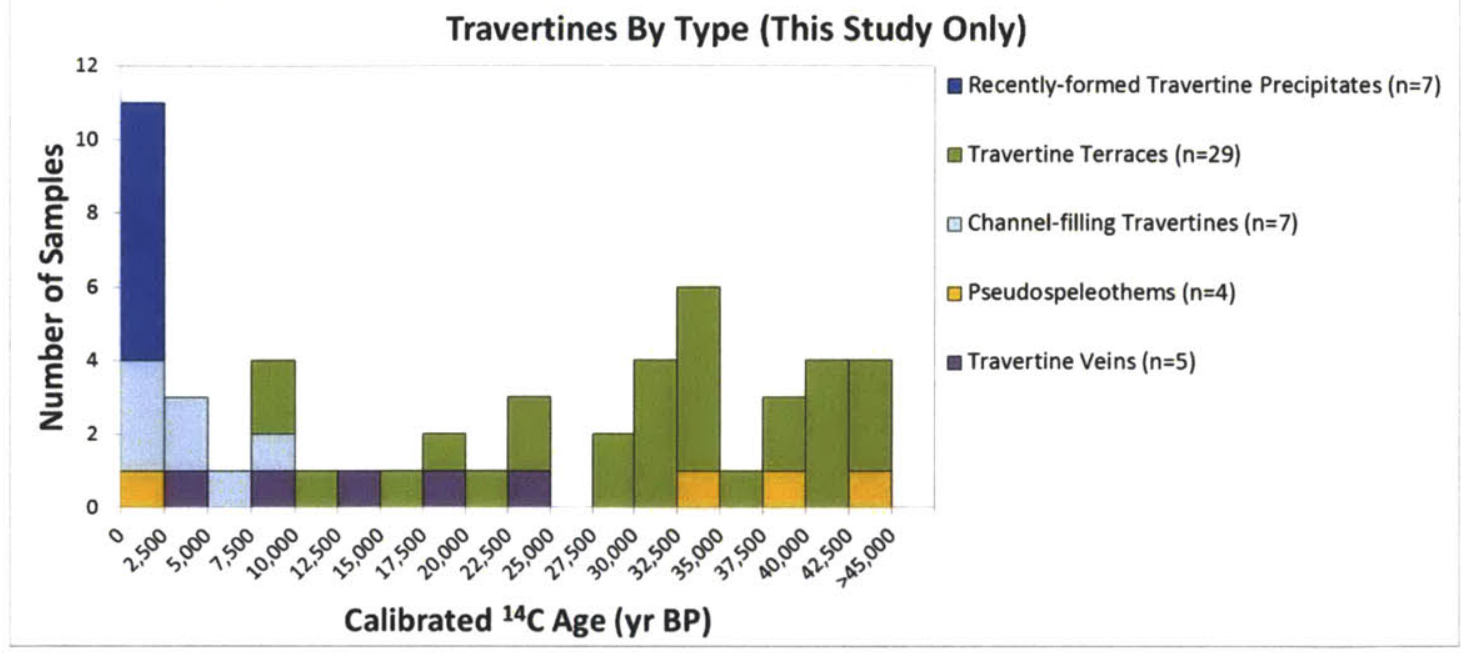

Figure 5: Distribution of ${ }^{14} \mathrm{C}$ ages for travertines from the Samail Ophiolite. The ${ }^{14} \mathrm{C}$ data were converted to calibrated ages using the method described in the text. 


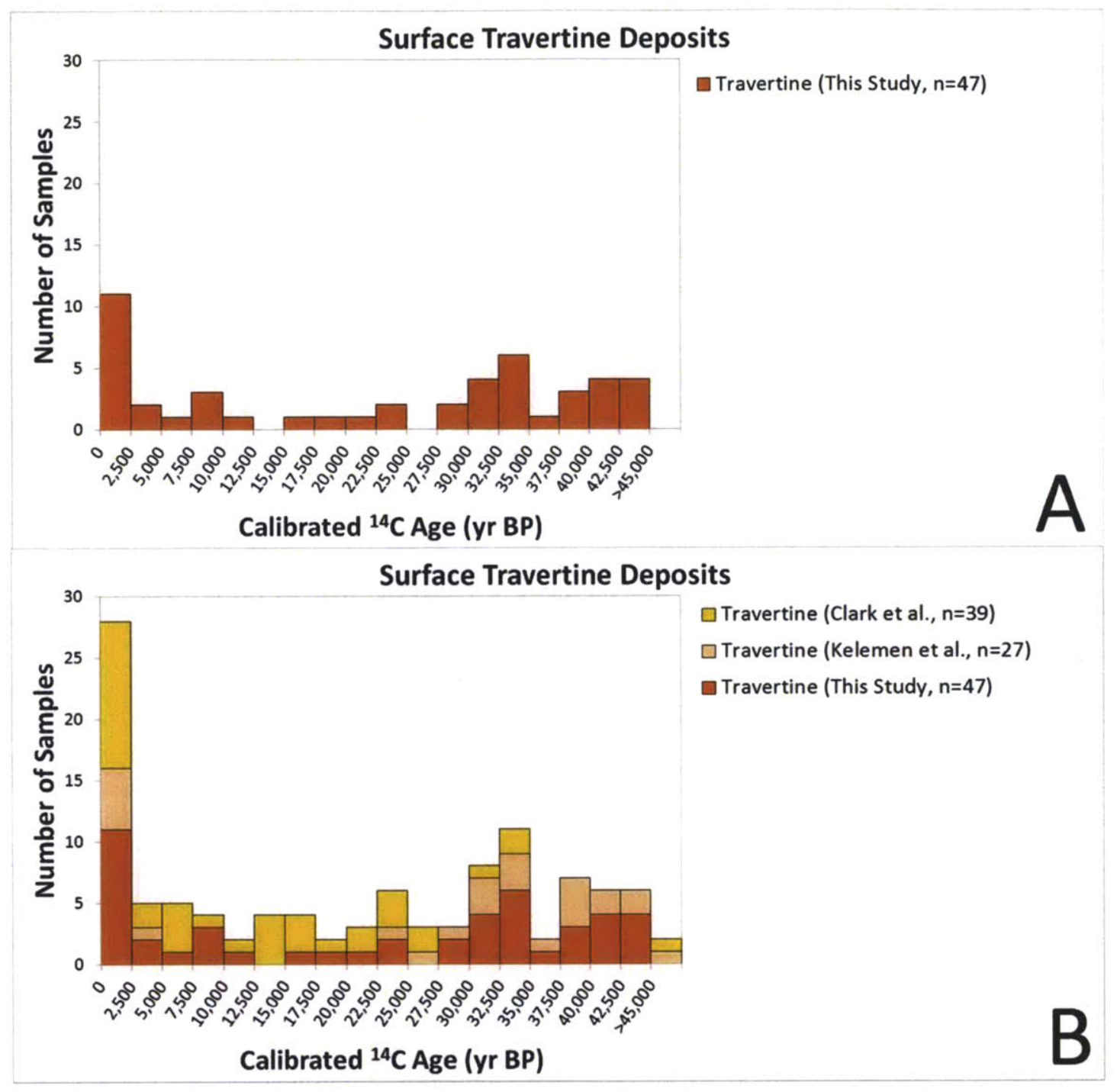

Figure 6: Distribution of ${ }^{14} \mathrm{C}$ ages for surface travertines from the Samail Ophiolite. A: Data from this study include the recently-formed travertine precipitates, travertine terraces, channel-filling travertines, and pseudospeleothems which are distinguished in Figure 5. B: All available ${ }^{14} \mathrm{C}$ ages: this study (dark orange bars); Kelemen and Matter (2008), Kelemen et al. (2011), and Kelemen et al., unpublished, (light orange bars); and Clark and Fontes (1990) and Clark et al. (1992) (yellow bars). Note that the ${ }^{14} \mathrm{C}$ data from this study and from Kelemen et al. were converted to calibrated ages using the method described in the text. Data from Clark et al. were calibrated using an older version of the ${ }^{14} \mathrm{C}$ caibration curve. Recalibration is not possible due to the unavailability of the uncalibrated data. 


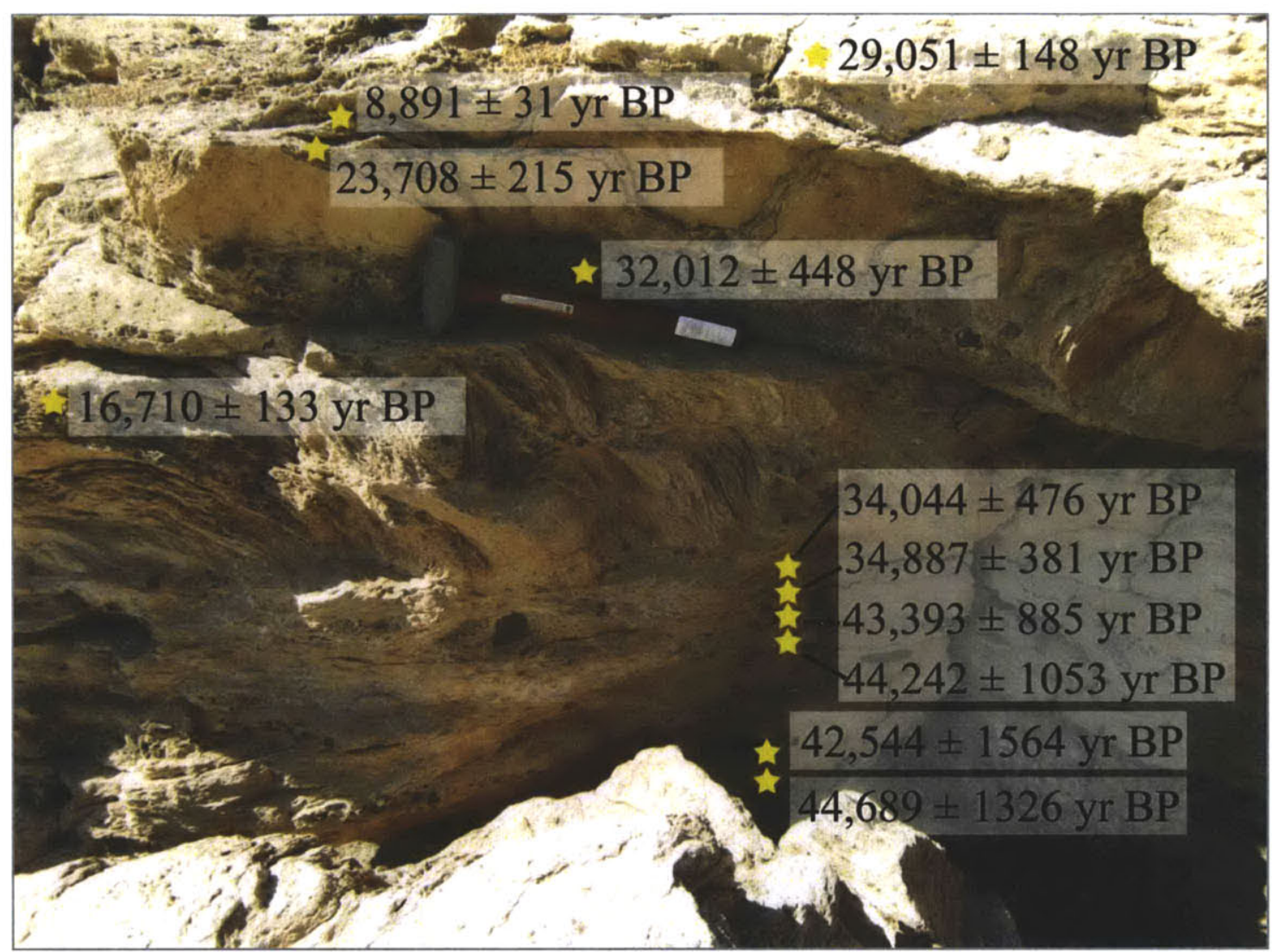

Figure 7: The subsampled travertine terrace at Misht Travertine. The travertine terrace is $\sim 2 \mathrm{~m}$ thick overall. The ${ }^{14} \mathrm{C}$ ages presented on the figure are calibrated ages that were determined using the method described in the text. 


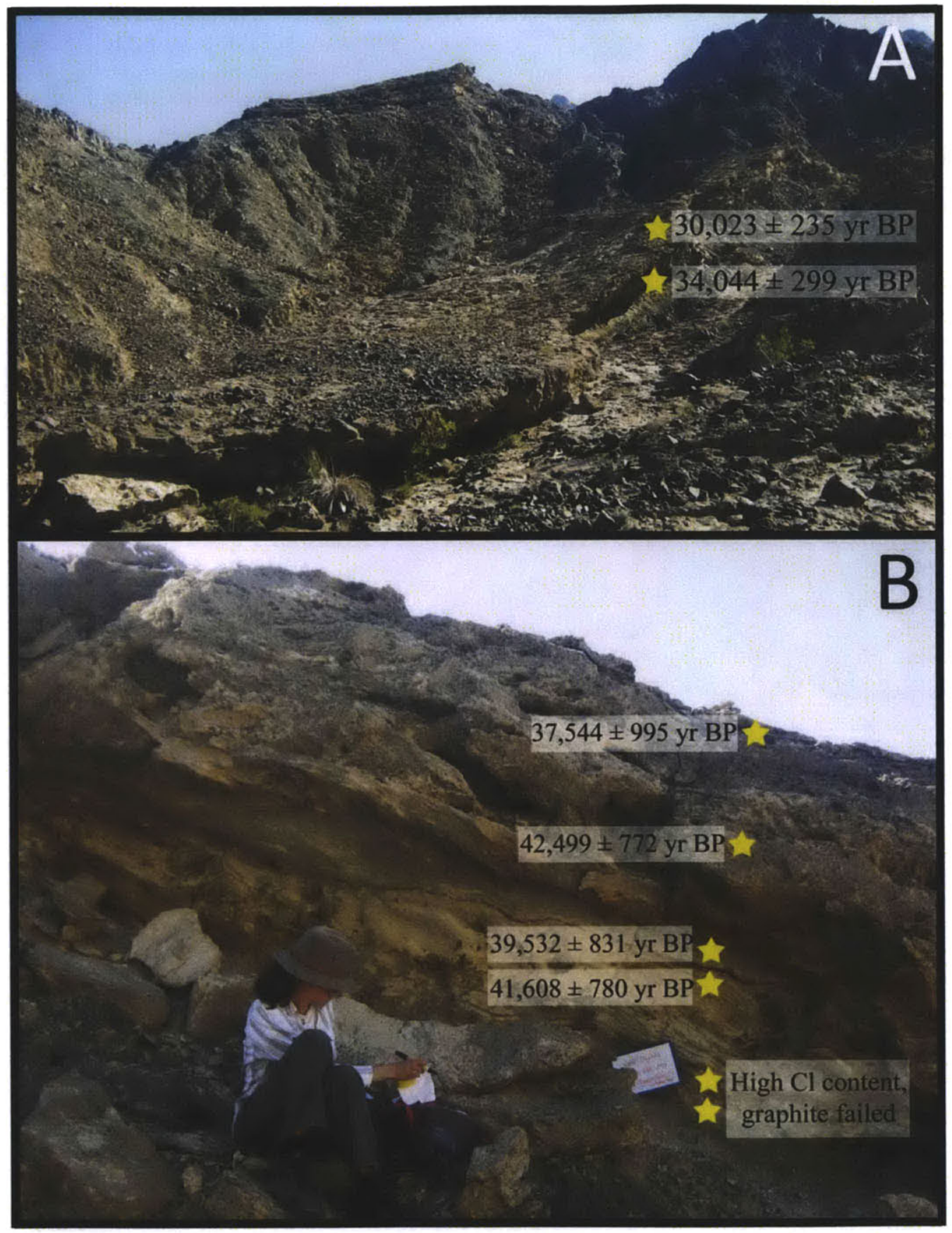

Figure 8: The two subsampled travertine terraces at Wadi Uqaybah Travertine. The terrace shown in $\mathrm{A}$ is $\sim 1 \mathrm{~m}$ thick while the terrace shown in $\mathrm{B}$ is closer to $2 \mathrm{~m}$ thick. The ${ }^{14} \mathrm{C}$ ages presented on the figure are calibrated ages that were determined using the method described in the text. 


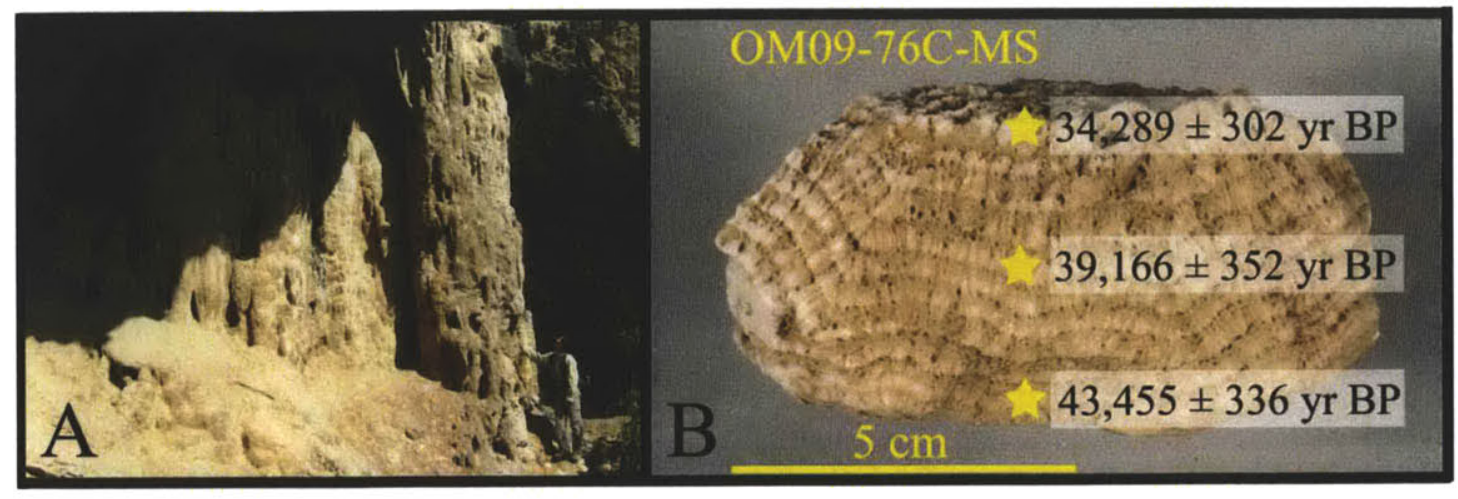

Figure 9: The subsampled travertine pseudospeleothem from Wadi Sudari Travertine. A: The pseudospeleothem sample was collected near the travertine column. Numerous pseudospeleothems are located in the overhang near the column; the column itself likely represents the joining of a pseudostalactite and a pseudostalagmite. B: A picture of the pseudospeleothem showing the locations of subsampling. The ${ }^{14} \mathrm{C}$ ages are calibrated ages that were determined using the method described in the text. 


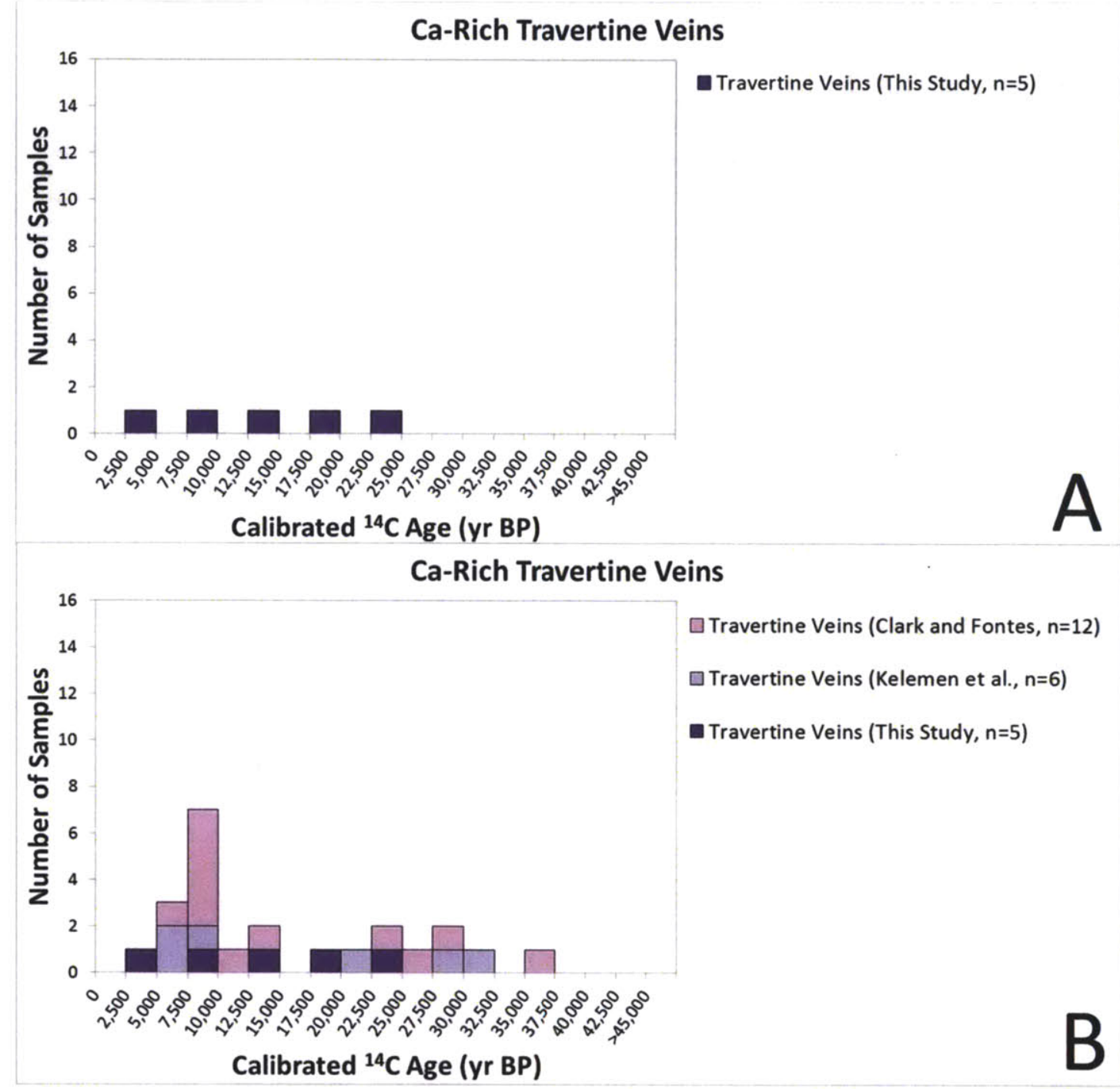

Figure 10: Distribution of ${ }^{14} \mathrm{C}$ ages for travertine veins from the Samail Ophiolite. A: Data from this study only. B: All available ${ }^{14} \mathrm{C}$ ages: this study (dark purple bars); Kelemen and Matter (2008), Kelemen et al. (2011), and Kelemen et al., unpublished, (light purple bars); and Clark and Fontes (1990) and Clark et al. (1992) (pink bars). Note that the ${ }^{14} \mathrm{C}$ data from this study and from Kelemen et al. were converted to calibrated ages using the method described in the text. Data from Clark et al. were calibrated using an older version of the ${ }^{14} \mathrm{C}$ caibration curve. Recalibration is not possible due to the unavailability of the uncalibrated data. 

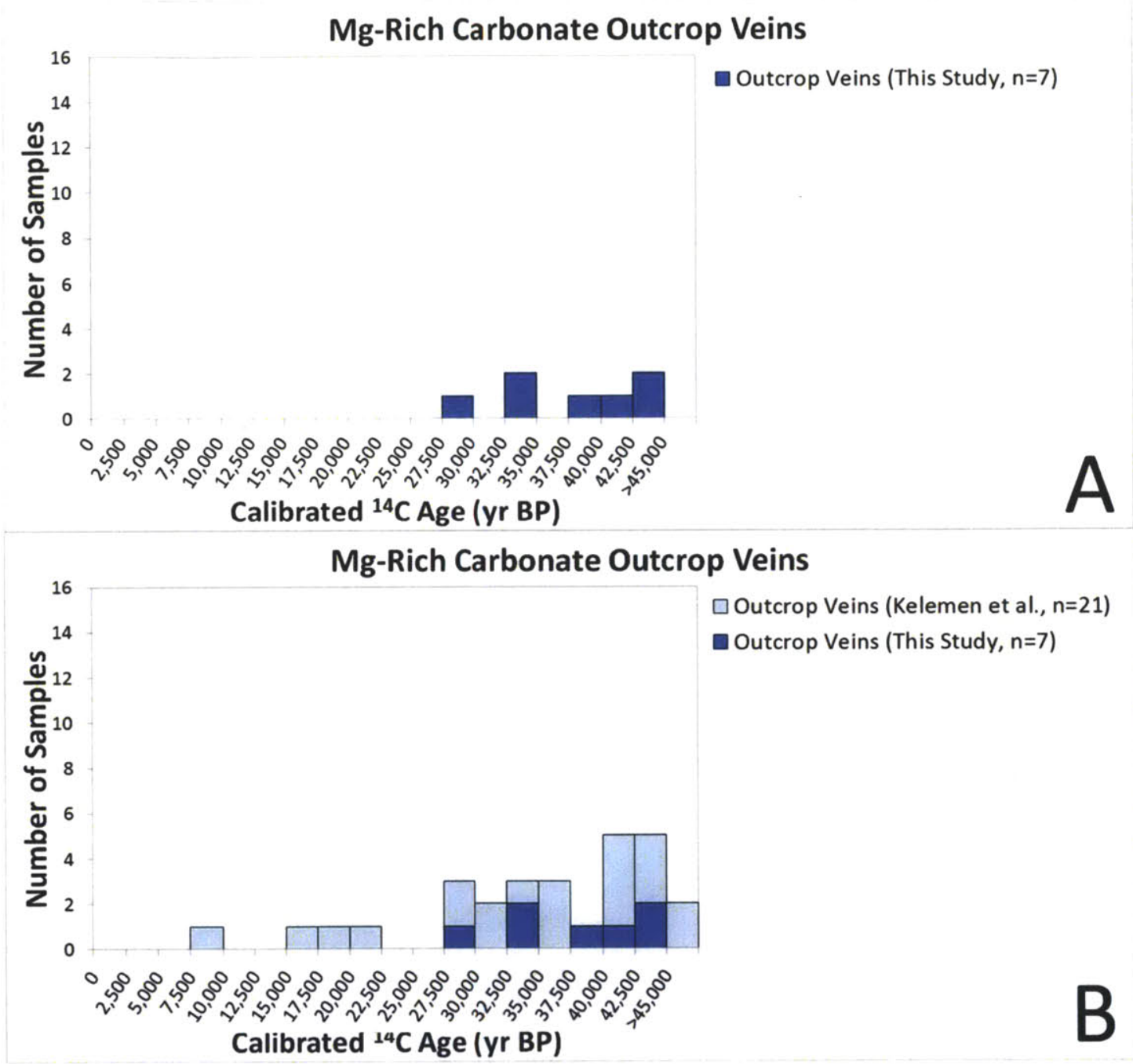

Figure 11: Distribution of ${ }^{14} \mathrm{C}$ ages for $\mathrm{Mg}$-rich carbonate outcrop veins from the Samail Ophiolite. A: Data from this study only. B: All available ${ }^{14} \mathrm{C}$ ages: this study (dark blue bars); Kelemen and Matter (2008), Kelemen et al. (2011), and Kelemen et al., unpublished, (light blue bars). The ${ }^{14} \mathrm{C}$ data from this study and from Kelemen et al. were converted to calibrated ages using the method described in the text. 


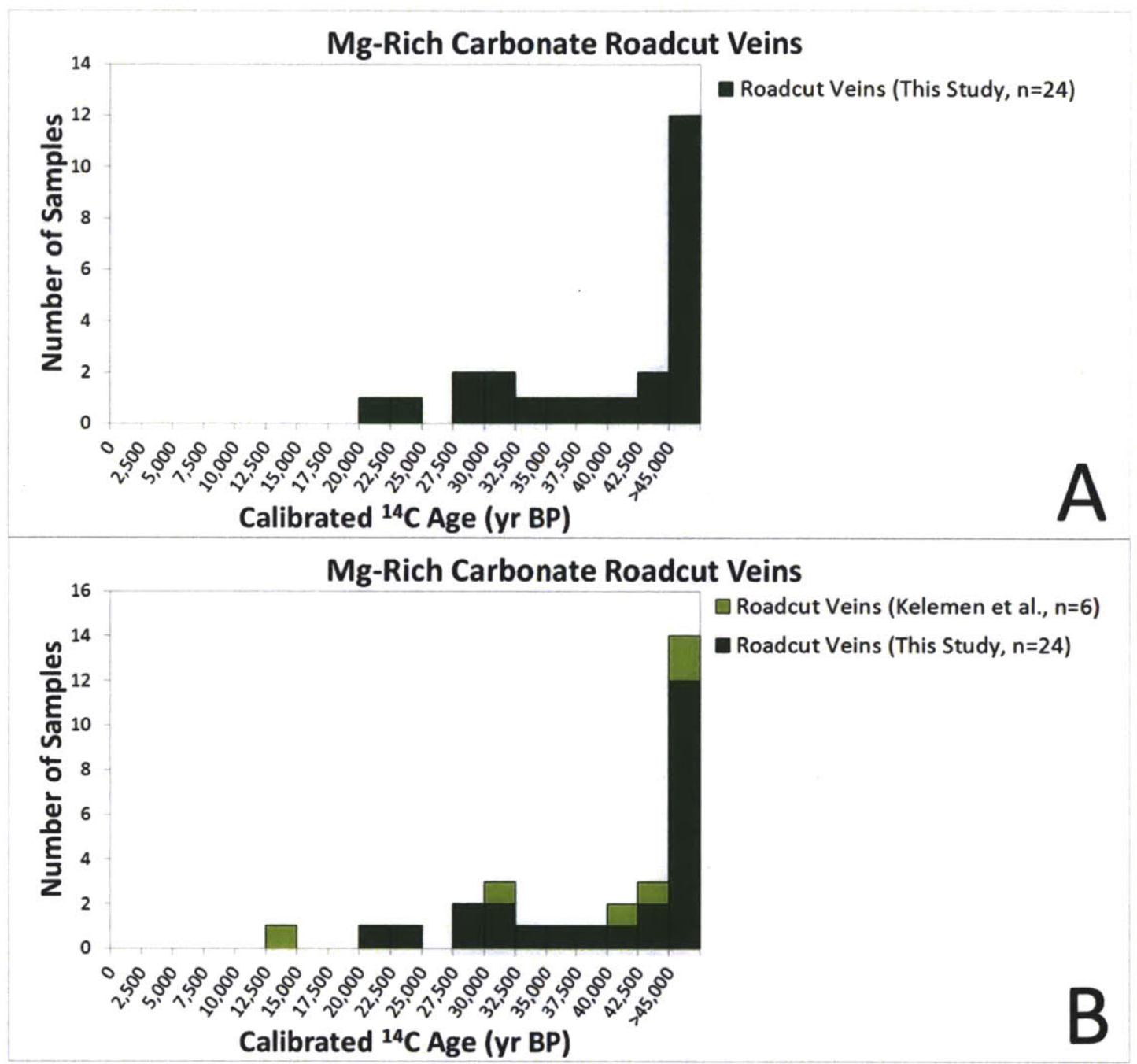

Figure 12: Distribution of ${ }^{14} \mathrm{C}$ ages for Mg-rich roadcut veins from the Samail Ophiolite. A: Data from this study only. B: All available ${ }^{14} \mathrm{C}$ ages: this study (dark green bars); Kelemen and Matter (2008), Kelemen et al. (2011), and Kelemen et al., unpublished, (light green bars). The ${ }^{14} \mathrm{C}$ data from this study and from Kelemen et al. were converted to calibrated ages using the method described in the text. 

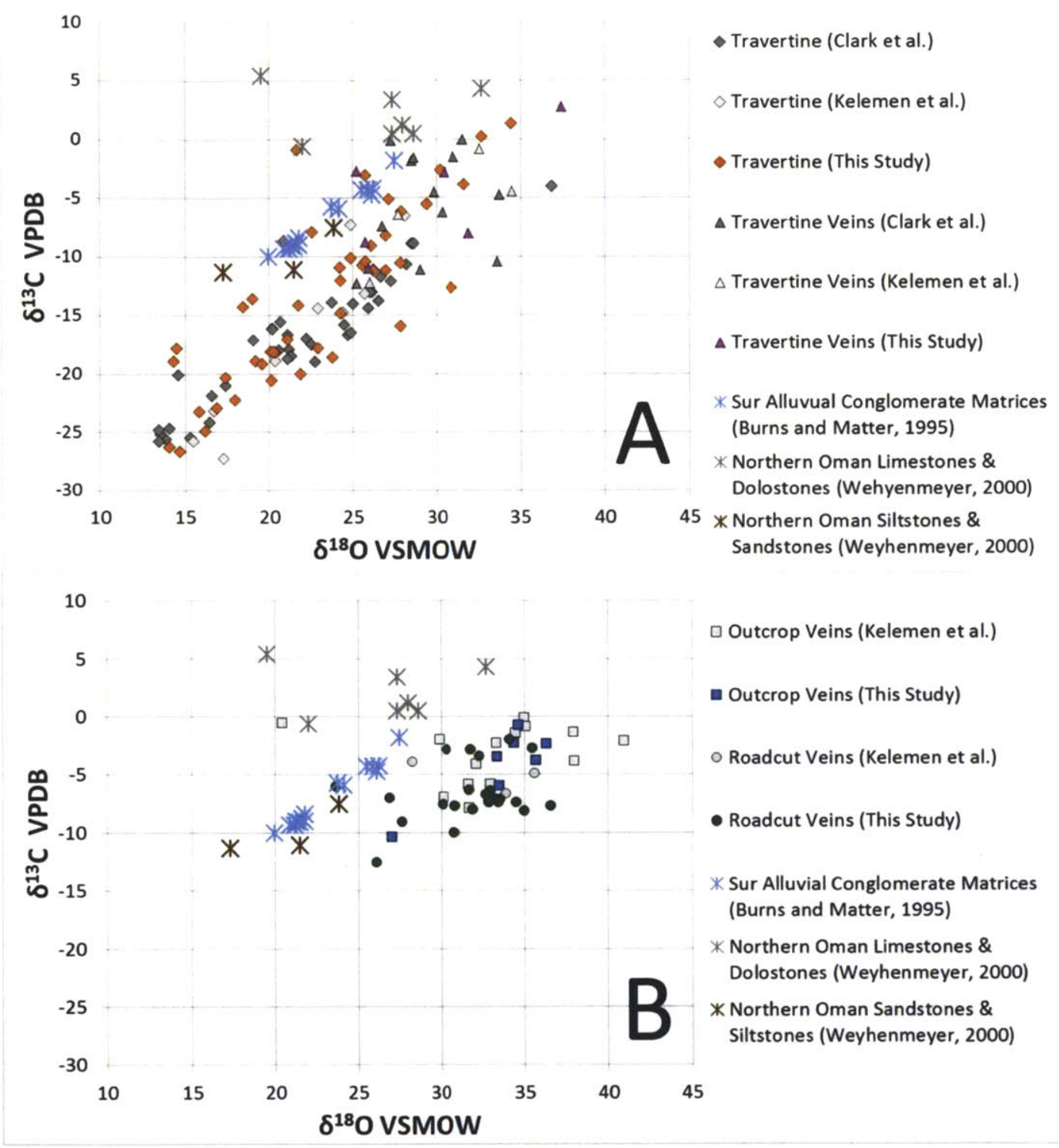

Figure 13: Relation between $\delta^{13} \mathrm{C}$ and $\delta^{18} \mathrm{O}$ isotopic ratios for Samail carbonates. The isotopic data are expressed in delta notation relative to the Vienna Pee Dee Belemite standard (VPDB) and Vienna Standard Mean Ocean Water (VSMOW). A: Ca-rich travertine veins (triangles) and surface travertine deposits (diamonds). B: Mg-rich carbonate outcrop veins (squares) and $\mathrm{Mg}$-rich carbonate roadcut veins (circles). Data from this study (colored symbols); Kelemen and Matter (2008), Kelemen et al. (2011), and Kelemen et al. (unpublished data) (light gray symbols); and Clark and Fontes (1990) and Clark et al. (1992) (dark gray symbols); Barns and Matter (1995) (light blue stars); and Weyhenmeyer (2000) (brown and gray stars). 

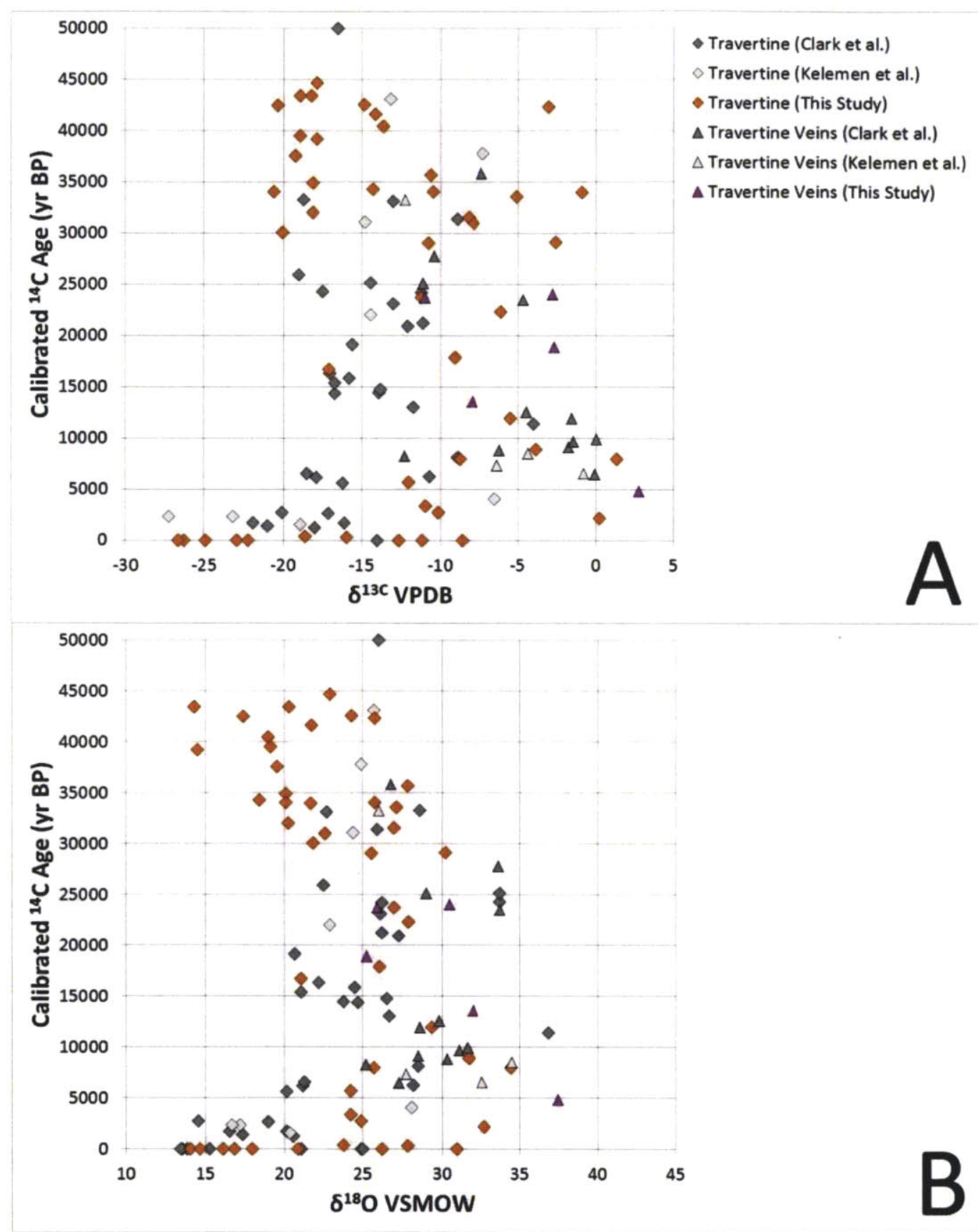

Figure 14. Relation between ${ }^{14} \mathrm{C}$ age and $\delta^{13} \mathrm{C}$ and $\delta^{18} \mathrm{O}$ isotopic ratios for surface travertines (diamonds) and travertine veins (triangles) from the Samail Ophiolite. The isotopic data are expressed in delta notation relative to the Vienna Pee Dee Belemite standard (VPDB) and Vienna Standard Mean Ocean Water (VSMOW). Symbols and data sources as in Figure 13A. 

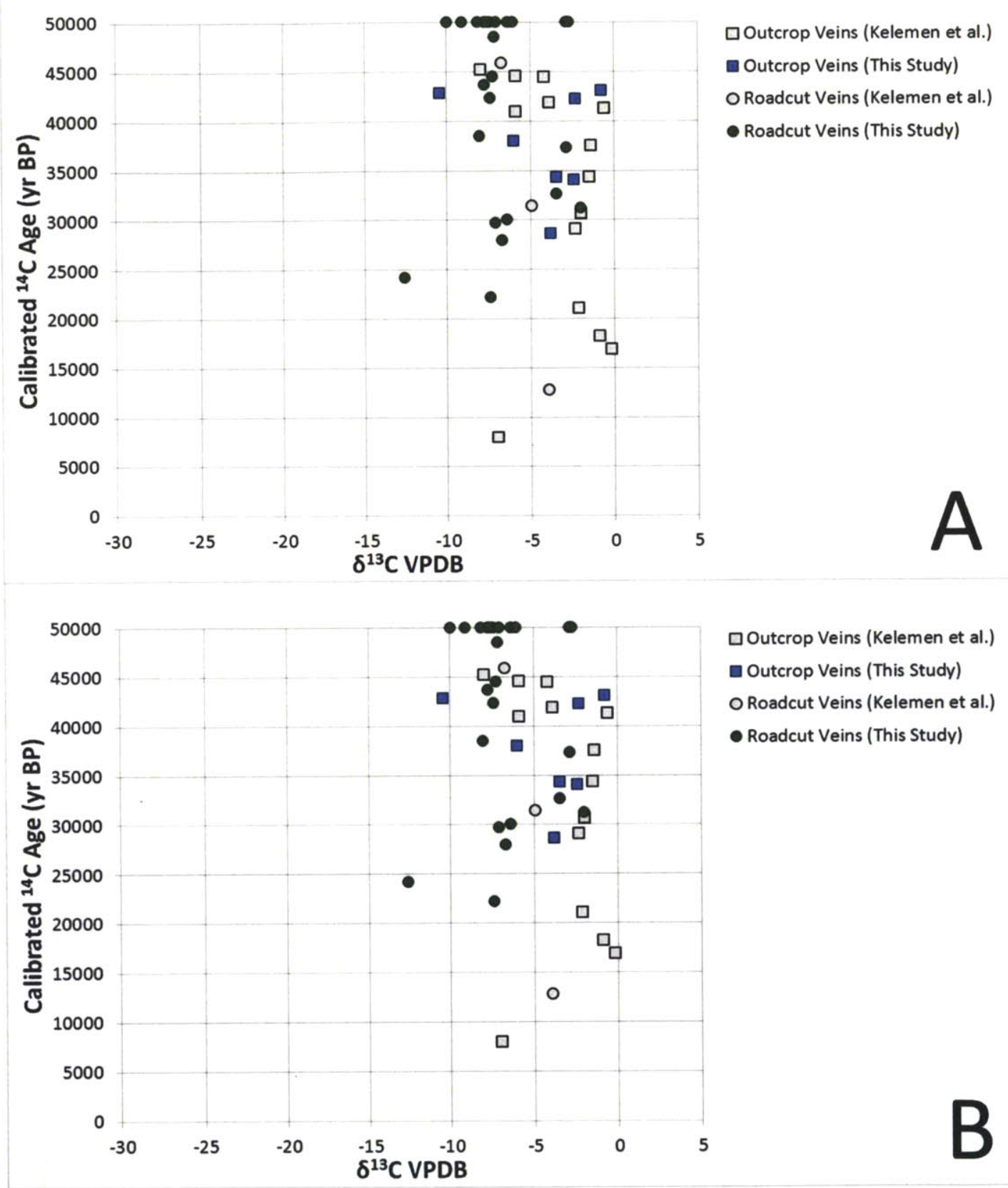

Figure 15. Relation between ${ }^{14} \mathrm{C}$ age and $\delta^{13} \mathrm{C}$ and $\delta^{18} \mathrm{O}$ isotopic ratios for Mg-rich outcrop (squares) and roadcut (circles) veins from the Samail Ophiolite. The isotopic data are expressed in delta notation relative to the Vienna Pee Dee Belemite standard (VPDB) and Vienna Standard Mean Ocean Water (VSMOW). Symbols and data sources as in Figure 13B. 


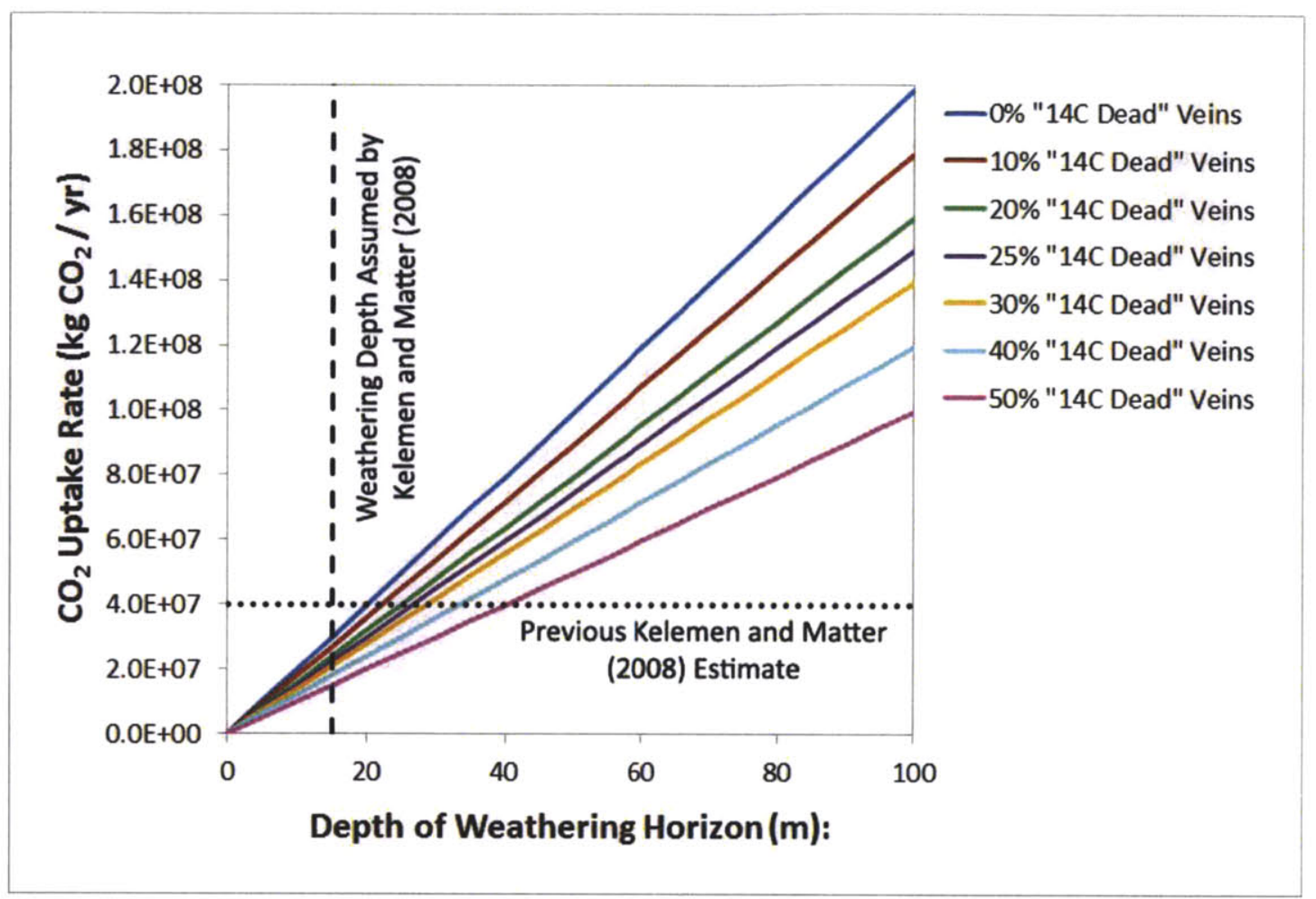

Figure 16: Sensitivity analysis illustrating the variation of $\mathrm{CO}_{2}$ uptake rates as a function of the depth of the weathering horizon and the percentage of ${ }^{14} \mathrm{C}$ dead subsurface veins in the Samail Ophiolite. 


\section{References:}

Akbulut, M., Piskin, O., and Karayigit, A.I. 2006. The genesis of the carbonatized and silicified ultramafics known as listwanites: A case study from the Mihaliccik region (Eskisehir), NW Turkey. Geological Journal, Vol. 41: 557-580.

Alt, J.C. and Teagle, D.A.H. 1999. The uptake of carbon during alteration of oceanic crust. Geochimica et Cosmochimica Acta, Vol. 63: 1527-1535.

Barnes, I., LaMarche, V.C., and Himmelberg, G. 1967. Geochemical evidence of present day serpentinization. Science, Vol. 156: 830-832.

Barnes, I. and O'Neil, J.R. 1969. The relationship between fluids in some fresh Alpine type ultramafics and possible modern serpentinization, Western United States. Geological Society of America Bulletin, Vol. 80: 1947-1960.

Barnes, I., O’Neil, J.R., and Trescases, J.J. 1978. Present-day serpentinization in New Caledonia, Oman, and Yugoslavia. Geochimica et Cosmochimia Acta, Vol. 42: 144-145.

Boaretto, E., Bryant, C., Carmi, I., Cook, G., Gulliksen, S., Harkness, D., Heinemeier, J., McClure, J., McGee, E., Naysmith, P., Possnert, G., Scott, M., van der Plicht, H., van Strydnock, M. 2002. Summary findings of the fourth international radiocarbon intercomparison (FIRI) (1998-2001). Journal of Quaternary Science, Vol. 17, No. 7: 633-637.

Boudier, F. and Coleman, R.G. 1981. Cross section through the peridotite in the Semail ophiolite. Journal of Geophysical Research, Vol. 86: 2573-2592.

Boudier, F., Ceuleneer, G., and Nicolas, A. 1988. Shear zones, thrusts, and related magmatism in the Oman ophiolite: initiation of thrusting at an ocean ridge. Tectonophysics, Vol. 151: 275-296.

Boudier, F. and Nicolas, A. 2007. Comment on "Dating the geologic history of Oman's Semail ophiolite: Insights from U-Pb geochronology" by, C.J. Warren, R.R. Parrish, D.J. Waters, and M.P. Searle. Contributions to Mineralogy and Petrology, Vol. 154: 111-113.

Bruni, J., Canepa, M., Chiodini, G., Cioni, R., Cipolli, F., Longinelli, A., Marini, L., Ottonello, G., and Zuccolini, M.V. 2002. Irreversible water-rock mass transfer accompanying the generation of the neutral, $\mathrm{Mg}-\mathrm{HCO}_{3}$ and high-pH, $\mathrm{Ca}-\mathrm{OH}$ spring waters of the Genova province, Italy. Applied Geochemistry, Vol. 17: 455474. 
Burke, A., Robinson, L.F., McNichol, A.P., Jenkins, W.J., Scanlon, K.M, and Gerlach, D.S. 2010. Reconnaissance dating: a new radiocarbon method applied to assessing the Temporal distribution of Southern Ocean deep-sea corals. Deep-Sea Research I, Vol. 57: 1510-1520.

Burns, S.J. and Matter, A. 1995. Geochemistry of carbonate cements in surficial alluvial conglomerates and their paleoclimatic implications. Journal of Sedimentary Research, Vol. A65, No. 1: 170-177.

Burns, S.J., Fleitmann, D., Matter, A., Neff, U., and Mangini, A. 2001. Speleothem evidence from Oman for continental pluvial events during interglacial periods. Geology, Vol. 29, No.7: 623-626.

Chacko, T. and Deines, P. 2008. Theoretical calculation of oxygen isotope fractionation factors in carbonate systems. Geochimica et Cosmochimica Acta, Vol. 72: 36423660 .

Chizmeshya, A.V.G., McKelvy, M.J., Squires, K., Carpenter, R.W., and Bérat, H. 2007. A novel approach to mineral carbonation: enhancing carbonation while avoiding mineral pretreatment costs. U.S. Department of Energy Final Report 924162, Arizona State University, Tempe, Arizona.

Cipolli, F., Gambardella, B., Marini, L., Otonello, G., and Zuccolini, M.V. 2004. Geochemistry of high-pH waters from serpentinites of the Gruppo di Voltri (Genova, Italy) and reaction path modeling of $\mathrm{CO}_{2}$ sequestration in serpentine aquifers. Applied Geochemistry, Vol. 19: 787-802.

Clark, I.D. and Fontes, J.-C. 1990. Paleoclimate reconstruction in northern Oman based on carbonates from hyperalkaline groundwaters. Quaternary Research, Vol. 33: 320-336.

Clark, I.D., Fontes, J.-C., and Fritz, P. 1992. Stable isotope disequilibria in travertine from high $\mathrm{pH}$ waters: Laboratory investigations and field observations from Oman. Geochimica et Cosmochimica Acta, Vol. 56: 2041-2050.

Coleman, R.G. 1981. Tectonic setting for ophiolite obduction in Oman. Journal of Geophysical Research, Vol. 86, No. B4: 2497-2508.

Das Sharma, S., Patil, D.J., and Gopalan, K. 2008. Temperature dependence of oxygen isotope fractionation of $\mathrm{CO}_{2}$ from magnesite-phosphoric acid reaction. Geochimica et Cosmochimica Acta, Vol. 66, No. 4: 589-593.

Dessert, C., Dupré, B., Françoise, Schott, J., Gaillardet, J., Chakrapani, G., and Bajpai, S. 2001. Erosion of Deccan Traps determined by river chemistry: impact on the 
global climate and the ${ }^{87} \mathrm{Sr} /{ }^{86} \mathrm{Sr}$ ratio of seawater. Earth and Planetary Science Letters, Vol. 188: 459-574.

Dessert, C., Dupré, B., Gaillardet, J., François, L., and Allègre, C. 2003. Basalt weathering laws and the impact of basalt weathering on the global carbon cycle. Chemical Geology, Vol. 202: 257-273.

Dewandel, B., Lachassagne, P., Bakalowicz, M., Weng, P., and Al-Malki, A. 2003. Evaluation of aquifer thickness by analyzing recession hydrographs: Application to the Oman ophiolite hard-rock aquifer. Journal of Hydrology, Vol. 274, No. 1-4: 248-269.

Dewandel, B., Lachassagne, P., and Qatan, A. 2004. Spatial measurements of stream baseflow, a relevant method for aquifer characterization and permeability evaluation: Application to a hard rock aquifer, Oman ophiolite. Hydrological Processes, Vol. 18, No. 17: 3391-3400.

Dewandel, B., Lachassagne, P., Boudier, F., Al-Hattali, S., Ladouche, B., Pinault, J.-L. and Al-Suleimani, Z. 2005. A conceptual and hydrogeological model of ophiolite hard-rock aquifers in Oman based on a multiscale and multidisciplinary approach. Hydrogeology Journal, Vol. 13, No. 5-6: 708-726.

Ferry, J.M. 1995. Fluid-flow during contact-metamorphism of ophicarbonate rocks in the Bergell-Aureole, Val-Malenco, Italian Alps. Journal of Petrology, Vol. 36: 10391053.

Fleitmann, D., Burns, S.J., Neff, U., Mangini, A., and Matter, A. 2003a. Changing moisture sources over the last 330,000 years in Northern Oman from fluidinclusion evidence in speleothems. Quaternary Research, Vol. 60: 223-232.

Fleitmann, D., Burns, S.J., Mudelsee, M., Neff, U., Kramers, J., Mangini, A., and Matter, A. $2003 \mathrm{~b}$. Holocene forcing of the Indian monsoon recorded in a stalagmite from southern Oman. Science, Vol. 300: 1737-1739.

Fleitmann, D., Burns, S.J., Mangini, A., Mudelsee, M., Kramers, J., Villa, I., Neff, U., Al-Subbary, A. A., Buettner, A., Hippler, D., and Matter, A. 2007. HoloceneITCZ and Indian monsoon dynamics recorded in stalagmites from Oman and Yemen (Socotra). Quaternary Science Reviews, Vol. 26: 170-188.

Friedman, I. and O'Neil, J.R. 1977. Compilation of stable isotope factors of geochemical interest. In: USGS Professional Paper 440-KK, Data of Geochemistry, ed. M. Fleischer, pp. KK1-12. Reston, VA: U.S. Geological Survey. 
Früh-Green, G.L., Kelley, D.S., Bernasconi, S.M., Karson, J.A., Ludwig, K.A., Butterfield, D.A., Boschi, C., and Proskurowski, G. 2003. 30,000 years of hydrothermal activity at the Lost City vent field. Science, Vol. 301: 495-498.

Fuchs, M. and Buerkert, A. 2008. A 20 ka sediment record from the Hajar Mountain range in N-Oman and its implication for detecting arid-humid periods on the southeastern Arabian Peninsula. Earth and Planetary Science Letters, Vol. 265: 546-558.

Garrido, C.J., Kelemen, P.B., and Hirth, G. 2001. Variation of cooling rate with depth in lower crust formed at an oceanic spreading ridge: Plagioclase crystal size distributions in gabbros from the Oman Ophiolite. Geochemistry, Geophysics, Geosystems, Vol 2: $21 \mathrm{pp}$.

Goldberg, D.S., Takahashi, T., and Slagle, A.L. 2008. Carbon dioxide sequestration in deep-sea basalt. Proceedings of the National Academy of Sciences, Vol. 105, No. 29: $9920-9925$.

Graven, H.D., Guilderson, T.P., and Keeling, R.T. 2012. Observations of radiocarbon in $\mathrm{CO}_{2}$ at seven global sampling sites in the Scripps flask network: Analysis of spatial gradients and seasonal cycles. Journal of Geophysical Research, Vol. 117, DO2302, 16 pp.

Gregory, R.T. and Taylor, H.P. 1981. An oxygen isotope profile in a section of Cretaceous ocean crust, Samail Ophiolite, Oman: Evidence for $\delta 180$ buffering of the oceans by deep $(>5 \mathrm{~km})$ seawater-hydrothermal circulation and mid-ocean ridges. Journal of Geophysical Research, Vol. 86, No. B4: 2737-2755.

Hacker, B. R. 1994. Rapid emplacement of young oceanic lithosphere: Argon geochronology of the Oman Ophiolite. Science, Vol. 265, No. 5178: 1563-1565.

Halls, C. and Zhao, R. 1995. Listvenite and related rocks: Perspectives on terminology and mineralogy with reference to an occurrence at Cregganbaun, Co. Mayo, Republic of Ireland. Mineralium Deposita, Vol. 30: 303-313.

Hansen, L.D., Dipple, G.M., Gordon, T.M., and Kellet, D.A. 2005. Carbonated serpentinite (listwanite) at Atlin, British Columbia: A geological analogue to carbon dioxide sequestration. Canadian Mineralogist, Vol. 43: 225-239.

Hua, Q. and Barbetti, M. 2004. Review of tropospheric bomb ${ }^{14} \mathrm{C}$ data for carbon cycle modeling and age calibration purposes. Radiocarbon, Vol. 46, No. 3: 1273-1298.

Ivany, L.C., Lohmann, K.C., Blake, D.B., Aronson, R.B. Hasiuk, F., Glass, A., and Moody, R.M. 2008. Eocene climate record of a high southern latitude continental 
shelf and implications for the biota: Seymour Island, Antarctica. Geological Society of America Bulletin, Vol. 120: 659-678.

Karlen, I., Olssoon, I.U., Kallburg, P., and Kilici, S. 1964. Absolute determination of the activity of two ${ }^{14} \mathrm{C}$ dating standards. Arkiv Geofysik, Vol. 4: 465-471.

Kelemen, P.B. and Matter, J.M. 2008. In situ carbonation of peridotite for $\mathrm{CO}_{2}$ storage. Proceedings of the National Academy of Sciences, Vol. 105, No.45: 17295-17300.

Kelemen, P.B., Matter, J.M., Streit, E.E., Rudge, J.F., Curry, W.B., and Blusztajn, J. 2011. Rates and mechanisms of mineral carbonation in peridotite: Natural processes and recipes for enhanced, in situ $\mathrm{CO}_{2}$ capture and storage. Annual Reviews of Earth and Planetary Sciences, Vol. 39: 545-576.

Kelley, D.S., Karson, J.A., Blackman, D.K., Früh-Green, G.L., Butterfield, D.A., Lilley, M.D., Olson, E.J., Schrenk, M.O., Roe, K.K., Lebon, G.T., and Rivizzigo, P. 2001. An off-axis hydrothermal vent field near the Mid-Atlantic Ridge at 3 degrees N. Nature, Vol. 412: 145-149.

Lackner, K.S., Wendt, C.H., Butt, D.P., Joyce, E.L., and Sharp, D.H. 1995. Carbon dioxide disposal in carbonate minerals. Energy, Vol. 20: 1153-1170.

Lackner, K.S. 2002. Carbonate chemistry for sequestering fossil carbon. Annual Review of Energy and the Environment, Vol. 27: 193-232.

Lippard, S.J., Shelton, A.W., and Gass, I.G. 1986. The Ophiolite of Northern Oman. The Geological Society, Memoir No. 11. London: Blackwell Scientific Publications, pp. 178.

Ludwig, K.A., Kelley, D.S., Butterfield, D.A., Nelson, B.K., and Früh-Green, G. L. 2006. Formation and evolution of carbonate chimneys at the Lost City hydrothermal field. Geochimica et Cosmochimica Acta, Vol. 70: 3625-2645.

Ludwig, K.A., Shen, C.-C., Kelley, D.S., Cheng, H., and Edwards, L.R. 2011. U-Th systematic and ${ }^{230} \mathrm{Th}$ ages of carbonate chimneys at the Lost City hydrothermal field. Geochmica et Cosmochimica Acta, Vol. 75: 1869-1888.

Matter, J.M., Waber, H.N., Loew, S., and Matter, A. 2006. Recharge areas and geochemical evolution of groundwater in an alluvial aquifer system in the Sultanate of Oman. Hydrogeology Journal, Vol. 14: 203-224.

Matter, J.M. and Kelemen, P.B. 2009. Permanent storage of carbon dioxide in geological reservoirs by mineral carbonation. Nature Geoscience, Vol. 2: 837-841. 
Matter. J.M., Kelemen, P.B., Paukert, A., Mervine, E.M., and Shock, E. 2012. Geochemical constraints on in situ mineral carbonation of mantle peridotite in the Samail Ophiolite, Oman. Presented at the International Conference on the Geology of the Arabian Plate and the Oman Mountains, Sultan Qaboos University, Muscat, Oman, January $7^{\text {th }}-9^{\text {th }}$.

McConnaughey, T. 1989a. ${ }^{13} \mathrm{C}$ and ${ }^{18} \mathrm{O}$ isotopic disequilibrium in biological carbonates: I. Patterns. Geochimica et Cosmochmica Acta, Vol. 53: 151-162.

McConnaughey, T. $1989 \mathrm{~b} .{ }^{13} \mathrm{C}$ and ${ }^{18} \mathrm{O}$ isotopic disequilibrium in biological carbonates: II. In vitro simulation of kinetic isotope effects. Geochimica et Cosmochmica Acta, Vol. 53: 163-171.

McNichol, A.P., Gagnon, A.R., Osborne, E.A., Hutton, D.L., von Reden, K.F., and Schneider, R.J. 1995. Improvements in procedural blanks at NOSAMS: Reflections of improvements in sample preparation and accelerator operation. Radiocarbon, Vol. 37, No. 3: 683-691.

Nasir, S., Al-Sayigh, A.R., Al-Harthy, A., Al-Kirbash, S., Al-Jaaidi, O., Musllam, A., Al Mishwat, A., and Al-Bu'saidi, S. 2007. Mineralogical and geochemical characterization of listwaenite from the Semail Ophiolite, Oman. Chemie der Erde, Vol. 67, No. 3: 213-228.

Neal, C. and Stanger, G. 1983. Hydrogen generation from mantle source rocks in Oman. Earth and Planetary Science Letters, Vol. 66: 315-320.

Neal, C. and Stanger, G. 1984. Calcium and magnesium-hydroxide precipitation from alkaline groundwaters in Oman, and their significance to the process of serpentinization. Mineralogical Magazine, vol. 48: 237-241.

Neal, C. and Stanger, G. 1985. Past and present serpentinization of ultramafic rocks: An example from the Semail ophiolite nappe of northern Oman. In: Drewer, J.I. (ed.), The Chemistry of Weathering. Holland: D. Reidel Publishing Company: 249-275.

Nicolas, A. 1989. Structures of ophiolites and dynamics of oceanic lithosphere. Amsterdam: Kluwer Academic Publishers, pp. 367.

Nicolas, A., Boudier, F., Ildefonse, V., and Ball, E. 2000. Accretion of Oman and United Arab Emirates ophiolite-Discussion of a new structural map. Marine Geophysical Researches, Vol. 21: 147-149.

O'Connor, W.K., Dahlin, D.C., Rush, G.E., Gerdemann, S.J., and Neilsen, D.N. 2004. Aqueous mineral carbonation. U.S. Department of Energy Final Report 
DOE/ARC-TR-04-002, U.S. Department of Energy Albany Research Center, Albany.

Olsson, I.U. 1970. The use of Oxalic acid as a standard. In: Radiocarbon Variations and Absolute Chronology, Nobel Symposium, 12 ${ }^{\text {th }}$ Proceedings. New York: John Wiley and Sons.

O’Neil, J.R., Clayton, R.N., and Mayeda, T.K. 1969. Oxygen isotope fractionation in divalent metal carbonates. Journal of Chemical Physics, Vol. 51: 5547-5558.

Pallister, J.S. and Knight, R.J. 1981. Rare-earth element geochemistry of the SamailOphiolite near Ibra, Oman. Journal of Geophysical Research, Vol. 86, No. B4: $\quad 2673-2697$.

Pallister, J.S. and Hopson, C.A. 1981. Samail Ophiolite plutonic suite: Field relations,phase variation, cryptic variation and layering, and a model of a spreading ridge magma chamber. Journal of Geophysical Research, Vol. 86, No. B4: 2593-2644.

Pearce, J.A., Alabaster, T., Shelton, A.W., and Searle, M.P. 1981. The Oman ophiolite as a Cretaceous arc-basin complex: Evidence and implications. Philosophical Transactions of the Royal Society of London, Series A: Mathematical and Physical Sciences, Vol. 300, No. 1454: 299-317.

Pentecost, A. 2005. Travertine. Berlin: Springer-Verlag, pp. 445.

Reimer, P.J., Baillie, M.G.L., Bard, E., Bayliss, A., Beck, J.W., Blackwell, P.G., Bronk, Ramsey, C., Buck, C.E., Burr, G.S., Edwards, R.L., Friedrich, M., Grootes, P.M., Guilderson, T.P., Hajdas, I., Heaton, T.J., Hogg, A.G., Hughen, K.A., Kaiser, K.F., Kromer, B., McCormac, F. G., Manning, S.W., Reimer, R. W., Richards, D.A., Southon, J.R., Talamo, S., Turney, C.S.M., van der Plicht, J., and Weyhenmeyer, C.E. 2009. INTCAL09 and MARINE09 radiocarbon age calibration curves, 0-50,000 years cal BP. Radiocarbon, Vol. 51, No. 4: 11111150 .

Ricou, L.E. 1971. Le croissant ophiolitique peri-Arabe, une ceinture de nappes mises en place au Cretace superieur. Revue de Geographie Physique et de Geologie Dynamique, Vol. 13, No. 4: 327-349.

Rioux, M., Bowring, S.A., and Kelemen, P.B. 2008. Timescales of crusal accretion at a medium to fast spreading ridge: High precision $\mathrm{U}-\mathrm{Pb}$ zircon dating of th intrusive crust of the Cretaceous Oman Ophiolite. Eos, Transactions, American Geophysical Union, Vol. 89, No. 53, Supplement, Abstract V44B-02. 
Roberts, M.L., Burton, J.R., Elder, K.L., Longworth, B.E., McIntyre, C.P., von Reden, K.F., Han, B.X., Rosenheim, B.E., Jenkins, W.J., Galutschek, E., and McNichol, A.P. 2009. A high-performance 14-C accelerator mass spectrumetry system. In: Proceedings of the $20^{\text {th }}$ International Radiocarbon Conference, Radiocarbon, Kona, $H I$.

Rozanski, K., Stichler, W., Gonfiantini, R., Scott, E.M., Beukens, R.P., Kromer, B., and van der Plicht, J.1992. The IAEA C-14 intercomparison exercise 1990. Radiocarbon, Vol. 34: 506-519.

Scott, E.M. 2003. The third international radiocarbon intercomparison (TIRI) and the fourth international radiocarbon intercomparison (FIRI) 1990-2002. Results, analyses, and conclusions. Radiocarbon, Vol. 45, no. 2: 135-408.

Searle, M.P. and Cox, J. 1999. Tectonic setting, origin, and obduction of the Oman ophiolite. Geological Society of America Bulletin, Vol. 111, No. 1: 104-122.

Searle, M.P. and Cox, J. 2002. Subduction zone metamorphism during formation and emplacement of the Semail ophiolite in the Oman Mountains. Geological Magazine, Vol. 139, No. 3: 241-255.

Searle, M.P. and Malpas, J. 1980. The structure and metamorphism of rocks beneath the Semail ophiolite of Oman and their significance in ophiolite obduction. Transactions of the Royal Society of Edinburgh, Vol. 71, No. 4: 247-262.

Searle, M.P. and Malpas, J. 1982. Petrochemistry and origin of sub-ophiolite metamorphic and related rocks in the Oman Mountains. Journal of the Geological Society of London, Vol. 139, No. 3: 235-248.

Seifritz, W. 1990. $\mathrm{CO}_{2}$ disposal by means of silicates. Nature, Vol. 345: 486.

Stanger, G. 1985. Silicified serpentinite in the Semail nappe of the Oman. Lithos, Vol. 18: 13-22.

Staudigel, H., Hart, S.R., Schmincke, H.-U., and Smith, B.M. 1989. Cretaceous ocean crust at DSDP Sites 417 and 418: Carbon uptake from weathering verses loss by magmatic outgassing. Geochimica et Cosmochimica Acta, Vol. 53: 3091-3094.

Stuiver, M., and Polach, H.A. 1977. Discussion: Reporting of ${ }^{14} \mathrm{C}$ Data. Radiocarbon, Vol. 19: 355-363.

Stuiver, M. 1980. Workshop on ${ }^{14} \mathrm{C}$ data reporting. Radiocarbon, Vol. 22: 964-966. 
Surour, A. A. and Arafa, E.H. 1997. Ophicarbonates: Calichified serpentinites from Gebel Mohagara, Wadi Ghadir area, Eastern Desert, Egypt. Journal of African Earth Sciences, Vol. 24: 315-324.

Tilton, G.R., Hopson, C.A., and Wright, J.E. 1981. Uranium-lead isotopic ages of the Samail Ophiolite, Oman, with applications to Tethyan Ocean Ridge Tectonics. Journal of Geophysical Research, Vol. 86, No. B4: 2763-2775.

Trommsdorff, V. and Evans, B.W. 1977. Antigorite-ophicarbonates: Contact metamorphism in Valmalenco, Italy. Contributions to Mineralogy and Petrology, Vol. 62: 301-312.

Trommsdorff, V., Evans, B.W., and Pfeifer, H.R. 1980. Ophicarbonate rocks:Metamorphic reactions and a possible origin. Archives des Sciences Genève, Vol. 33: 361-364.

Vadpalas, B., Weidman, C., and Cronin, E.K. 2011. Validation of age estimation in geoduck clams using the bomb radiocarbon signal. Journal of Shellfish Research, Vol. 30, No 2: 1-5.

VanTongeren, J.A., Kelemen, P.B., and Hangh øj, K. 2008. Cooling rates in the lowe crust of the Oman Ophiolite: $\mathrm{Ca}$ in olivine, revisited. Earth and Planetary Science Letters, Vol. 267: 69-82.

Vogel, J.S., Nelson, D.E., and Southon, J.R. 1987. ${ }^{14} \mathrm{C}$ background levels in an accelerator mass spectrometry system. Radiocarbon, Vol. 29, No. 3: 323-333.

Warren, C.J., Parrish, R.R., Searle, M.P., and Waters, D.J. 2003. Dating the subduction of the Arabian continental margin beneath the Semail ophiolite. Geology, Vol. 31, 889-892.

Warren, C.J., Parrish, R.R., Waters, D.J., and Searle, M.P. 2005. Dating the geologic history of Oman's Semail Ophiolite: Insights from U-Pb geochronology. Contributions to Mineralogy and Petrology, Vol. 150: 403-422.

Warren, C.J., Searle, M.P., Parrish, R.R., and Waters, D.J. 2007. Reply to comment by F. Boudier and A. Nicolas on "Dating the geologic history of Oman's Semail Ophiolite: Insights from U-Pb geochronology” by C.J. Warren, R.R. Parrish, M.P. Searle, and D.J. Waters. Contributions to Mineralogy and Petrology, Vol. 154: 115-118.

Weyhenmeyer, C.E. 2000. Origin and evolution of groundwater in the alluvial aquifer of the eastern Batinah coastal plain, Sultanate of Oman: A hydrogeological approach. PhD Thesis. University of Bern, Switzerland, pp. 202. 
Wilde, A., Simpson, L., and Hanna, S. 2002. Preliminary study of Tertiary hydrothermal alteration and platinum deposition in the Oman ophiolite. Journal of the Virtual Explorer, Vol. 6: 7-13.

Wilson, S.A., Raudseep, M., and Dipple, G.M. 2006. Verifying and quantifying carbon fixation in minerals from serpentine-rich mine tailings using the Rietveld method with X-ray powder diffraction data. American Mineralogist, Vol. 91, No. 8-9: 1331-1341.

Wilson, S.A., Raudsepp, M., and Dipple, G.M. 2009a. Quantifying carbon fixation in trace minerals from processed kimberlite: A comparative study of quantitativemethods using X-ray powder diffraction data with applications to the Diavik Diamond Mine, Northwest Territories, Canada. Applied Geochemistry, Vol. 24, No. 12: 95-112.

Wilson, S.A., Dipple, G.M., Power, I.M., Thom, J.M., Anderson, R.G., Raudsepp, M., Gabites, J.E., and Southam, G. 2009b. Carbon dioxide fixation within mine wastes of ultramafic-hosted ore deposits: Examples from the Clinton Creek and Cassiar chrysotile deposits, Canada. Economic Geology, Vol. 104, No. 1: 95-1 12.

Wilson, S.A., Barker, S.L.L., Dipple, G.M., and Atudorei, V. 2010. Isotopic disequilibrium during uptake of atmospheric $\mathrm{CO}_{2}$ into mine process waters: Implications for $\mathrm{CO}_{2}$ sequestration. Environmental Science and Technology, Vol. 44, No. 24: 9522-9529.

Xu, X.M., Trumbore, S.E., Zheng, S.H., Southon, J.R., McDuffee, K.E., Luttgen, M., andLiu, J.C. 2007. Modifying a sealed tube zinc reduction method for preparation of AMS graphite targets: reducing background and attaining high precision. Nuclear Instruments and Methods in Physics Research Section B-Bean Interactions with Materials and Atoms, Vol. 259, No. 1: 320-329. 


\title{
Chapter 3: ${ }^{230}$ Th Dating of Carbonate Alteration Products of Peridotite in the Samail Ophiolite, Sultanate of Oman: Applications and Limitations
}

\begin{abstract}
:
${ }^{230} \mathrm{Th}$ dating was attempted on carbonate alteration products forming in the peridotite layer of the Samail Ophiolite, Sultanate of Oman in order to further refine constraints on natural rates of carbonate formation in peridotite. Due to their low $U$ concentrations $(<1 \mathrm{ppb}-1 \mathrm{ppm})$ and relatively high $\mathrm{Th} / \mathrm{U}$ ratios $(0.01-5.6$; average: 1.3$)$, Samail carbonates are challenging to date with the ${ }^{230} \mathrm{Th}$ technique due to the sensitivity of the ages to corrections for initial ${ }^{230} \mathrm{Th}$. Uncorrected ${ }^{230} \mathrm{Th}$ ages for Ca-rich travertines are consistently older than previously obtained ${ }^{14} \mathrm{C}$ ages. However, geologically reasonable initial ${ }^{230} \mathrm{Th}$ corrections for the ${ }^{230} \mathrm{Th}$ ages bring the two sets of ages into concordance. In contrast, uncorrected ${ }^{230} \mathrm{Th}$ ages for Mg-rich carbonate veins sampled on the natural peridotite weathering surface are generally younger than previously obtained ${ }^{14} \mathrm{C}$ ages. This is likely due to the addition of excess ${ }^{238} \mathrm{U}$ to the carbonate outcrop veins during alteration by reducing serpentinization fluids. Two Mg-rich carbonate veins sampled from roadcuts have near-equilibrium $\left({ }^{230} \mathrm{Th} /{ }^{238} \mathrm{U}\right)$ and $\left({ }^{234} \mathrm{U} /{ }^{238} \mathrm{U}\right)$, which indicates that these veins are $>375,000$ years in age, consistent with their " ${ }^{14} \mathrm{C}$ dead" $(>50,000 \mathrm{yr}$ BP) ages.

This study presents some of the first $U$ and Th isotopic measurements for peridotites. Highly-altered peridotites have excess ${ }^{238} U$, which is most likely the result of $\mathrm{U}$ addition during serpentization under reducing conditions within the last 375,000 years. The highly-altered peridotites also have similar $\left({ }^{234} U /{ }^{238} U\right)$ enrichments to the carbonate outcrop veins, which suggest that both types of samples have been altered by a similar
\end{abstract}


fluid. In contrast, the more pristine peridotite standard PCC-1 and dunite standard DTS-1 have $\left({ }^{234} \mathrm{U} /{ }^{238} \mathrm{U}\right)$ values which are close to equilibrium.

\section{Introduction:}

Determining timescales of the formation and preservation of carbonate alteration products in mantle peridotite is important in order to better understand the role of this potentially important sink in the global carbon cycle and also to evaluate the feasibility of using artificially-enhanced, in situ formation of carbonates in peritdotite as a method for mitigating the buildup of anthropogenic $\mathrm{CO}_{2}$ emissions in the atmosphere. The goal of this study is to determine ${ }^{230} \mathrm{Th}$ ages for Samail carbonates in order to further assess the range of ages of carbonate alteration products in the Samail Ophiolite, Sultanate of Oman. This study builds upon previous ${ }^{14} \mathrm{C}$ dating and stable $\mathrm{C}$ and $\mathrm{O}$ isotope analyses of Samail carbonates, which are presented in Chapter 2 of this thesis and also in Clark and Fontes (1990), Kelemen and Matter (2008), and Kelemen et al. (2011). ${ }^{230} \mathrm{Th}$ dating, which has a practical limit of $\sim 375,000$ years, greatly extends the dating range of ${ }^{14} \mathrm{C}$ dating, which has a practical limit of $\sim 50,000$ years. In addition, combined ${ }^{14} \mathrm{C}$ and ${ }^{230} \mathrm{Th}$ dating allows greater confidence to be placed in the radiometric ages where the two dating techniques are concordant and permits the investigation of open-system behavior where the two dating techniques are discordant. Due to the low $\mathrm{U}$ concentrations and high $\mathrm{Th} / \mathrm{U}$ ratios found in Samail carbonates, determining precise ${ }^{230} \mathrm{Th}$ ages for these carbonates is challenging and highly sensitive to initial ${ }^{230}$ Th corrections. Nevertheless, comparison of ${ }^{230} \mathrm{Th}$ and ${ }^{14} \mathrm{C}$ ages and age limits, as well as investigation of $\mathrm{U}$ and $\mathrm{Th}$ 
systematics during alteration of peridotite, provides important constraints on the timescales of natural carbonate formation in the peridotite layer of the Samail Ophiolite.

\section{Geologic Setting:}

The Samail Ophilote (Figure 1) is located in the Sultanate of Oman and the United Arab Emirates and is one of the largest and best-exposed ophiolites in the world. The ophiolite consists of Cretaceous oceanic crust $(4-7 \mathrm{~km})$ and mantle $(8-12 \mathrm{~km})$ that was uplifted and emplaced along with the Hawasina pelagic sedimentary formation (Glennie et al., 1973, 1974; Coleman, 1981; Lippard et al., 1986; Nicolas et al., 2000). The ophiolite is approximately $50-100 \mathrm{~km}$ in width and extends for greater than $500 \mathrm{~km}$ in length (Lippard et al., 1986; Nicolas et al., 2000). A more detailed discussion of the geology of the Samail Ophiolite is presented in Chapter 2 and also in the summaries found in Glennie et al. (1973, 1974), Coleman (1981), Pearce et al. (1981), Lippard et al. (1986), Nicolas (1989), Hacker et al. (1996), Searle and Cox (1999), and Nicolas et al. (2000).

This study focuses on ${ }^{230} \mathrm{Th}$ dating of two types of carbonate alteration products that form in the peridotite layer of the Samail Ophiolite: (1.) travertine precipitated from high pH springs (Clark and Fontes, 1990; Kelemen and Matter, 2008; Kelemen et al., 2011), and (2.) carbonate veins that form in situ in partially-hydrated (serpentinized) peridotite (Kelemen and Matter, 2008; Kelemen et al., 2011). As illustrated in Figure 2, these carbonates are thought to form as a result of low-temperature alteration of peridotite through interaction with meteoric water (e.g. Barnes et al., 1967, 1978; Barnes and O’Neil, 1969; Bruni et al., 2002; Cipolli et al., 2004; Kelemen et al., 2011). When 
meteoric water weathers partially-serpentinized peridotite, $\mathrm{Mg}^{2+}-\mathrm{HCO}_{3}^{-}$rich waters (known as "Type I" waters) are formed. When these waters percolate deeper into the peridotite bedrock where they are no longer in equilibrium with the atmosphere, they precipitate Mg-rich carbonates, serpentines, and clays. As a result of this precipitation and other reactions in the subsurface, the waters transform into $\mathrm{Ca}^{2+}-\mathrm{OH}^{-}$waters $($known as "Type II" waters) which have very high $\mathrm{pH}(10-12)$ and low oxygen fugacity (Eh approximately -200 mV) (e.g. Neal and Stanger, 1983, 1984, 1985; Clark and Fontes, 1990; Kelemen and Matter, 2008; Kelemen et al., 2011). When the Type II waters return to the surface as hyperalkaline springs, they rapidly react with atmospheric $\mathrm{CO}_{2}$ to precipitate calcite-rich travertines (e.g. Clark and Fontes, 1990; Clark et al., 1992; Kelemen and Matter, 2008).

\section{Sample Descriptions:}

Carbonate (Figure 3) and peridotite (Figure 4) samples were obtained from eight locations in the Samail Ophiolite (Figure 1): four locations of travertine deposition (Qafeefah Travertine, Misht Travertine, Wadi Uqaybah Travertine, and Wadi Sudari Travertine), three roadcuts exposing carbonate veins in peridotite (Qafeefah Roadcut, Fanja Roadcut, and Al-Wuqbah Roadcut), and one campsite located on the natural peridotite weathering surface and containing abundant carbonate veins (Wadi Sudari Campsite). Sample descriptions are presented in Table 1. All carbonates analyzed for U and Th were also dated with ${ }^{14} \mathrm{C}$ and analyzed for $\delta^{13} \mathrm{C}$ and $\delta^{18} \mathrm{O}$, the results of which are presented in Chapter 2. 
Uranium and Th measurements were obtained for three types of surface travertines (Figure 3): travertine terraces (OM09-106C-MS-A, OM09-106C-MS-B, OM09-107C-MS, and OM09-109C-MS), recently-formed travertine precipitates sampled from hyperalkaline pools (OM09-8COPS-MS and OM09-10COPS-MS), and travertine pseudospeleothems (OM09-76C-MS-A and OM09-76C-MS-C). Pseudospeleothems are speleothem-like formations that have developed in overhangs, such as underneath travertine terraces. The two pseudospeleothem samples analyzed in this study were subsampled from a single layered pseudospleothem "drop" which had fallen from a travertine overhang at Wadi Sudari that contained abundant stalactite- and stalagmitetype travertines. In addition, $\mathrm{U}$ and $\mathrm{Th}$ measurements were obtained for one Ca-rich travertine vein (OM09-84C-MS).

Uranium and Th measurements were also obtained for thirteen Mg-rich carbonate veins (Figure 3). For the purposes of this study, these veins are divided into "outcrop veins" (sampled from the natural peridotite weathering surface) and "roadcut veins" (sampled at roadcuts). Both types are believed to be $\mathrm{Mg}$-rich veins that formed in the deeper subsurface; however, the roadcut veins are generally less-weathered than the outcrop veins, which are often partially eroded. Uranium and Th concentration measurements were also obtained for two serpentine mineral fractions (OM10-27C-MG and OM10-54C-MG) which were separated out of intergrown serpentine-carbonate veins sampled at roadcuts.

To investigate the composition of Samail peridotites, which are a possible source of detritus in the carbonates, $U$ and Th measurements were obtained for six highly-altered 
peridotites (Figure 4) collected from the natural peridotite weathering surface at locations of abundant carbonate formation. Two of these peridotites (OM10-1P-MG and OM102P-MG) were collected from the natural peridotite weathering surface located at the top of Fanja Roadcut. Three of these peridotites (OM10-13P-MG, OM10-17P-MG, and OM10-19P-MG) were collected nearby surface travertine deposits while the last peridotite (OM10-22P-MG) was collected adjacent to the carbonate outcrop veins sampled at Wadi Sudari Campsite. In order to compare the highly-altered peridotites with less-altered peridotites, $\mathrm{U}$ and $\mathrm{Th}$ measurements were also obtained for two harzburgites (OM94-74h and OM94-104) and a dunite (OM94-74d) from the Wadi Tayin massif of the Samail Ophiolite. These three peridotites were previously analyzed for $\mathrm{U}$ and Th by Hanghøj et al. (2010). In addition, $U$ and Th measurements were obtained for the USGS standards PCC-1 (peridotite) and DTS-1 (dunite).

Finally, $\mathrm{U}$ and $\mathrm{Th}$ measurements were obtained for two sediment samples, which are another potential source of detritus in the carbonates, collected from small wadis or valleys adjacent to the carbonates sampled at Fanja Roadcut and Wadi Uqaybah Travertine. There is no significant soil formation at any of the sampling locations, so these sediments consist of loose detritus that contains fragments of peridotite and carbonate as well as aeolian particles.

\section{Methods:}

Carbonates selected for $U$ and $T h$ analysis were made into chips using a jaw crusher and then purified through magnetic separation and hand-picking. Many of the carbonate veins consist of multiple minerals (e.g. magnesite, calcite, dolomite, and 
serpentine). Whenever possible, monomineralic (or nearly so) mineral separates were prepared, and their purity was determined by X-ray diffraction analysis at WHOI. While large fragments of peridotite and serpentine were easily removed through magnetic separation and hand-picking, fine-grained aluminosilicate material incorporated into the travertines and, to a lesser extent, into the subsurface carbonate veins was impossible to remove through physical separation. Note that the two recently-formed travertine precipitates (OM09-8COPS-MS and OM09-10COPS-MS) were not purified (aside from removing large, obvious peridotite clasts) due to their fine-grained nature.

For travertines, approximately 1-2 grams of material was required for each sample. For Mg-rich carbonate veins, approximately 20-30 grams of material was required for each sample because of extremely low $\mathrm{U}$ and $\mathrm{Th}$ concentrations. For select Mg-rich carbonate veins as well as the two serpentine mineral separates, approximately 1-2 grams of material was first analyzed for $U$ and Th concentrations in order to identify samples that were most promising for isotopic analysis (e.g. had the highest $U$ concentrations). For the peridotites and sediments, 2-10 grams of a bulk powder was analyzed for each sample. The mineral compositions of the highly-altered peridotites and sediments were determined by X-ray diffraction analysis at WHOI.

Carbonates selected for ${ }^{230} \mathrm{Th}$ dating were completely dissolved using $\mathrm{HNO}_{3}$, $\mathrm{HF}$, $\mathrm{HClO}_{4}$, and $\mathrm{H}_{3} \mathrm{BO}_{3}$. To investigate the behavior of samples during dissolution and also the nature of the incorporated aluminosilicate detritus, separate weak $\mathrm{HNO}_{3}$ leaches were carried out for select samples. The aluminosilicate residues left behind by these weak $\mathrm{HNO}_{3}$ leaches were also dissolved and analyzed separately. For one travertine sample 
(OM09-107C-MS), a progressive dissolution experiment was carried out. These progressive dissolutions are discussed in detail in Appendix A. In brief, the dissolution experiments indicate that both elemental and isotopic fractionation of $U$ and Th occurs during partial dissolutions of Samail carbonates. This fractionation is most significant for the surface travertines, which incorporate higher amounts of aluminosilicate detritus than the subsurface carbonate veins. The observed fractionation in the partial dissolutions reinforces the need to employ a total dissolution approach for ${ }^{230} \mathrm{Th}$ dating of "dirty" carbonates such as the Samail carbonates (e.g. Bischoff and Fitzpatrick, 1991; Luo and $\mathrm{Ku}, 1991)$.

After dissolution, $\mathrm{U}$ and Th were purified using two columns (see details in Ball et al., 2008 and Sims et al., 2008a). The first column was a nitric anion column that removes $\mathrm{U}$ and $\mathrm{Th}$ from the rock matrix. The second column was a hydrochloric anion column that separates $\mathrm{U}$ from $\mathrm{Th}$. For large samples, $\mathrm{U}$ and Th were co-precipitated with Fe hydroxide. For the Fe hydroxide precipitation, $3.5 \mathrm{mg}$ of an ultrapure Fe ICP standard manufactured by Ricca Chemical was added to each sample.

Uranium and Th concentrations were measured by isotope dilution ICP-MS using ${ }^{229} \mathrm{Th}$ and ${ }^{233} \mathrm{U}$ spikes. The ID measurements were carried out on a Thermo Element2 Mass Spectrometer at WHOI following procedures detailed in Sims et al. (2008a). U and Th isotopes were measured by MC-ICP-MS on the ThermoFinnegan NEPTUNE at WHOI using techniques described in Ball et al. (2008) and Sims et al. (2008a, b).

For quality assurance, silicate rock standards (ATHO and BHVO-1) and a carbonate standard (JCp-1) were analyzed for $\mathrm{U}$ and Th concentrations and isotopes. In 
addition, the ultramafic standards PCC-1 and DTS-1 were analyzed for U and Th concentrations and $U$ isotopes (Th isotopes could not be measured due to low Th concentrations). To our knowledge, this is the first time that these standards have been analyzed for $\mathrm{U}$ and $\mathrm{Th}$ concentrations by isotope dilution and for $\mathrm{U}$ isotopes.

\section{Results:}

\subsection{Mineralogy:}

Results of X-ray diffraction analysis of powdered samples are presented in Table 1. Mineral peaks were identified as "major" or "trace" but were not further quantified.

The travertine samples consist predominantly of calcite, often with trace quantities of unidentified clay minerals. One of the recently-formed travertine precipitates (OM09-8COPS-MS) also contains trace amounts of aragonite and possibly hydromagnesite while the other recently-formed travertine precipitate (OM09-12S-MS) contains trace quartz, presumably from aeolian sand that was incorporated into the sample when it formed. The Mg-rich subsurface veins consist primarily of magnesite or dolomite except for one sample (OM10-84C-MG) which consists primarily of brucite.

The highly-altered peridotites are predominantly composed of the serpentine minerals lizardite and chrysotile. Three of the highly-altered peridotites (OM10-1P-MG, OM10-13P-MG, and OM10-19P-MG) are almost entirely composed of serpentine. The other highly-altered peridotites also contain significant olivine and pyroxene. Some of the highly-altered peridotites also contain spinel and veins of carbonate and talc. The two serpentine mineral separates (OM10-27C-MG-SERP and OM10-54C-MG-SERP) are both pure lizardite. 
The two sediment samples have different mineral compositions. The sample collected near Fanja Roadcut (OM09-8S-MS) consists primarily of lizardite with trace amounts of quartz, dolomite, magnesite, and calcite. The other sediment sample collected near Wadi Uqaybah Travertine (OM09-12S-MS) consists primarily of quartz and calcite with trace chrysotile, dolomite, enstatite, and possibly aragonite. The difference in mineralogy reflects the different detrital inputs from the nearby environment and also from aeolian sources at these two locations.

\subsection{U and Th Concentrations:}

$\mathrm{U}$ and Th concentration data are presented in Table 2 and in Figure 5. Samail carbonates have $\mathrm{U}$ and $\mathrm{Th}$ concentrations ranging over several orders of magnitude from $<1 \mathrm{ppb}-\sim 1 \mathrm{ppm}$. The travertine terraces and wadi sediments have the highest $\mathrm{U}$ and $\mathrm{Th}$ concentrations, which range from $\sim 0.1-1 \mathrm{ppm}$ for both $\mathrm{U}$ and $\mathrm{Th}$.

The Mg-rich carbonate veins have $\mathrm{U}$ and Th concentrations ranging from $<1 \mathrm{ppb}$ $\sim 10 \mathrm{ppb}$, which is three to four orders of magnitude lower than the travertine $\mathrm{U}$ and Th concentrations. The lowest concentration Mg-rich carbonate vein (OM09-63C-MS) is below the instrument detection limit and has $\mathrm{U}$ and $\mathrm{Th}$ intensities comparable to the total procedural blank. The two serpentine mineral separates have very low $U$ and $T h$ concentrations $<1 \mathrm{ppb}$ which are comparable with those of the Mg-rich carbonate veins.

Samail peridotites also have low $\mathrm{U}$ and Th concentrations. The highly-altered peridotites analyzed in this study have $U$ and Th concentrations ranging from $\sim 1-100$ ppb. Five out of the six highly-altered peridotites have similar U concentrations $\sim 30-40$ ppb but cover a wider range of Th concentrations from $\sim 10-100 \mathrm{ppb}$. Sample OM10-1P- 
MG, which is the most highly-altered peridotite and has been almost completely serpentinized, has a significantly lower $U$ concentration of $\sim 4 \mathrm{ppb}$ and Th concentration of $\sim 3 \mathrm{ppb}$. The three less-altered peridotites from Wadi Tayin have Th concentrations of $\sim 1-10 \mathrm{ppb}$ which fall within the range of Th concentrations observed in the more highlyaltered peridotites. However, they have significantly lower $U$ concentrations of $<1 \mathrm{ppb}$. The U measurement for Sample OM94-74h is below the instrument detection limit and comparable to the total procedural blank. Note that the higher and more uniform $U$ and Th analyses previously obtained for the three Wadi Tayin peridotites by Hanhøj et al. (2010) were at the detection limit for $U$ and Th analyses by the ICP-MS technique employed in that study (Marguerite Godard, personal communication, 2012). The peridotite standard PCC-1 and the dunite standard DTS-1 have U and Th concentrations which agree well with the accepted values (e.g. Govindaraju, 1994). The standards have similar Th concentrations to the highly-altered peridoties. However, they have order of magnitude lower $U$ concentrations.

Three water samples from alkaline springs were prepared for $U$ and Th concentration analysis by isotope dilution. These water samples all had high $\mathrm{pH}$ (close to 12) and were collected from alkaline pools at Wadi Sudari Travertine, Misht Travertine, and Wadi Dima (near Qafeefah) during January 2009 by Jürg Matter. The water samples were filtered and acidified with ultrapure $\mathrm{HNO}_{3}$. All three water samples are well below the detection limits for $\mathrm{U}$ and $\mathrm{Th}$, which are determined by the ultrapure $\mathrm{HNO}_{3}$ blank. Approximately 50 additional water samples collected during 2009 and 2010 field seasons were analyzed for U and Th by ICP-MS (Jürg Matter and Everett Shock, personal 
communication, 2011). These water samples were obtained in the vicinity of alkaline springs and include both highly alkaline water $(\mathrm{pH} 10-12)$ and fresher wadi water $(\mathrm{pH}$ $\sim 8-10$ ). All of the water samples have $U$ and Th concentrations below the $0.5 \mathrm{ppb}$ calibration limit for ICP-MS, and the vast majority of the samples are below the detection limit of $0.001 \mathrm{ppb}$ (Everett Shock, personal communication, 2011). A few water samples have detectable $U$ and Th concentrations, but most of the analyses are just slightly above the field blank, which ranged from 0.001-0.003 ppb (Everett Shock, personal communication, 2011). The extremely low (essentially zero) U and Th concentrations prohibit further analysis of Samail waters for $\mathrm{U}$ and Th concentrations and isotopic compositions.

\section{$5.3 \mathrm{U}$ and Th Isotopes:}

$\mathrm{U}$ and Th isotopic data are presented in Table 3. Note that in the tables and throughout this paper, parentheses around isotopic ratios indicate activities. Due to the low $U$ and Th concentrations, only a subset of the samples analyzed for $U$ and Th concentrations could be analyzed for $U$ and Th isotopes. Figure 6 illustrates that many of the Samail carbonates and peridotites are out of equilibrium with respect to $\left({ }^{230} \mathrm{Th} /{ }^{238} \mathrm{U}\right)$. On this figure, the ${ }^{230} \mathrm{Th}$ and ${ }^{238} \mathrm{U}$ activities have been normalized by the ${ }^{232} \mathrm{Th}$ activities. Samples with $\left({ }^{230} \mathrm{Th} /{ }^{238} \mathrm{U}\right)=1$ (indicating isotopic equilibrium) plot on the 1 -to-1 line, which is called the equiline. All of the peridotite samples, four of the Mg-rich carbonate outcrop veins, two of the travertine terraces, and the travertine vein plot to the right of the equiline and thus have ${ }^{238} \mathrm{U}$ excesses. One travertine terrace, the two travertine 
pseudospeleothems, the two recently-formed travertine precipitates, four Mg-rich roadcut veins, and the two sediment samples fall either on or very close to the equiline.

Most of the Samail carbonates and peridotites are also out of equilibrium with respect to $\left({ }^{234} \mathrm{U} /{ }^{238} \mathrm{U}\right)$ (Figure 7). The travertine terraces, recently-formed travertine precipitates, and travertine pseudospeleothems all have ${ }^{234} U$ excesses with $\left({ }^{234} U /{ }^{238} U\right)$ activities between 1.1 and 1.3. However, the travertine vein plots on the other side of the equiline with $\left({ }^{234} \mathrm{U} /{ }^{238} \mathrm{U}\right)$ of 0.832 . The $\mathrm{Mg}$-rich carbonate outcrop veins have a fairly narrow range of $\left({ }^{234} \mathrm{U} /{ }^{238} \mathrm{U}\right)$ around 1.2. The Mg-rich carbonate roadcut veins have lower $\left({ }^{234} \mathrm{U} /{ }^{238} \mathrm{U}\right)$ values than the outcrop veins. Two of the roadcut veins have equilibrium $\left({ }^{234} U /{ }^{238} U\right)$ values. The highly-altered peridotites have ${ }^{234} U$ excesses except for sample OM10-1P-MG, which also has a significantly lower $U$ concentration (Table 2 and Figure 4) than the other highly-altered peridotite samples. The dunite standard DTS-1 has an equilibrium $\left({ }^{234} \mathrm{U} /{ }^{238} \mathrm{U}\right)$ value while the peridotite standard PCC-1 plots slightly above the equiline with $\left({ }^{234} U /{ }^{238} U\right)=1.045$. Sediment sample OM09-8S-MS plots just above the equiline with $\left({ }^{234} \mathrm{U} /{ }^{238} \mathrm{U}\right)=1.032$ while sediment sample OM09-12S-MS, which contains significant calcite that likely derives from nearby travertines, has higher $\left({ }^{234} \mathrm{U}{ }^{238} \mathrm{U}\right)=$ 1.102 , which is close to the values observed in the surface travertines.

Figure 8 illustrates the range of $\left({ }^{230} \mathrm{Th} /{ }^{232} \mathrm{Th}\right)$ values observed in Samail carbonates, peridotites, and wadi sediments. Most samples have $\left({ }^{230} \mathrm{Th} /{ }^{232} \mathrm{Th}\right)$ values of $\sim 0-5$. However, two samples have significantly higher $\left({ }^{230} \mathrm{Th} /{ }^{232} \mathrm{Th}\right)$ : highly-altered peridotite OM10-13C-MG has $\left({ }^{230} \mathrm{Th} /{ }^{232} \mathrm{Th}\right)=12.367$, and Mg-rich carbonate roadcut vein OM09-57C-MS has $\left({ }^{230} \mathrm{Th} /{ }^{232} \mathrm{Th}\right)=30.515$. 


\section{Discussion:}

\subsection{Systematics of $U$ and $T h$ in Samail Carbonates, Peridotites, and}

Sediments:

\subsection{1: Systematics of $U$ and Th Concentrations:}

Relative to other types of carbonates that are dated using the ${ }^{230} \mathrm{Th}$ method, the Samail carbonates have low $\mathrm{U}$ concentrations and much higher $\mathrm{Th} / \mathrm{U}$ ratios (Figure 5). The Samail carbonates have $U$ concentrations ranging from $<1 \mathrm{ppb}-\sim 1 \mathrm{ppm}$ (average: 0.1 $\mathrm{ppm}$ ) and $\mathrm{Th} / \mathrm{U}$ ratios ranging from 0.01-5.6 (average: 1.3). As a comparison, speleothems dated from Hoti Cave, which formed in Cretaceous limestone in Northern Oman, have $U$ concentrations ranging from $\sim 0.1-9 \mathrm{ppm}$ (average: $2 \mathrm{ppm}$ ) and $\mathrm{Th} / \mathrm{U}$ ratios ranging from $\sim 6 \times 10^{-5}$ to 0.06 (average: 0.01) (Fleitmann et al., 2003). Similarly, carbonates formed in peridotite at the Lost City hydrothermal field, which is located offaxis along the Mid-Atlantic Ridge, have $U$ concentrations ranging from $\sim 0.3-11 \mathrm{ppm}$ (average: $4 \mathrm{ppm}$ ) and $\mathrm{Th} / \mathrm{U}$ ratios ranging from $2 \times 10^{-5}-0.2$ (average: 0.01 ) (Ludwig, 2008; Ludwig et al., 2011). The higher U concentrations of the Lost City carbonates relative to the Samail carbonates are due to the incorporation of $U$ from seawater (Ludwig, 2008; Ludwig et al., 2011). As will be explained further in Section 6.2 below,

the high $\mathrm{Th} / \mathrm{U}$ ratios of the Samail carbonates are problematic for ${ }^{230} \mathrm{Th}$ dating because the determined ages are highly sensitive to initial ${ }^{230} \mathrm{Th}$ detrital corrections.

The low $U$ concentrations of the Samail carbonates reflect the low $U$ concentrations of the peridotites which are altering to form these carbonates (Figure 5). The Mg-rich veins, including the serpentine separates from carbonate-serpentine veins, 
have $U$ concentrations which are similar to the range of $U$ concentrations observed in the less-altered peridotites and the minimally altered standards PCC-1 (peridotite) and DTS-1 (dunite). However, the Mg-rich veins have lower U concentrations than all of the highlyaltered peridotites (except for OM10-1P-MG). The Mg-rich subsurface veins have lower Th concentrations than all of the peridotites. This is consistent with the concept that, under almost all conditions, Th is insoluble (e.g. Langmuir and Herman, 1980; Porcelli and Swarzenski, 2003). Therefore, the fluids responsible for the precipitation of the subsurface carbonates are unlikely to leach significant Th from the peridotite bedrock.

The higher U concentrations of the highly-altered peridotites (except for OM101P-MG) relative to the less-altered peridotites and also the PCC-1 and DTS-1 standards (Figure 5) are notable. The highly-altered peridotites contain significant serpentine minerals. Serpentinization fluids are known to have low oxygen fugacity and elevated dissolved hydrogen and methane (e.g. Barnes and O'Neil, 1969; Haggerty, 1991; Peretti et al., 1992; Kelley et al., 2005; Lang et al., 2010), and such fluids have been observed in the peridotite layer of the Samail Ophiolite (Neal and Stanger, 1983). Under reducing conditions, $\mathrm{U}$ converts from its soluble hexavalent form to its insoluble tetravalent form (e.g. Gascoyne, 1992; Porcelli and Swarzenski, 2003). Therefore, addition of U from reducing serpentinizing fluids may explain the enrichment of $U$ in the altered peridotites. Given that the two serpentine mineral separates have extremely low $\mathrm{U}$ concentrations and the most highly-serpentinized peridotite OM10-1P-MG (which consists of almost pure lizardite) has lower $U$ concentrations than the other highly-altered peridotites, the added 
$\mathrm{U}$ is likely being not being incorporated into serpentine but rather into other alteration minerals such as clays, talc, and perhaps carbonates.

The travertine samples have higher $\mathrm{U}$ and Th concentrations than the Mg-rich carbonate veins (Figure 5). The higher $U$ and $T h$ ratios in the travertines are likely a result of the incorporation of higher amounts of aluminosilicate detritus. There are multiple lines of evidence to support this interpretation. First, while the higher U concentrations of the travertines could possibly be explained by $U$.enrichment in fluids due to low water/rock ratios, the Th concentrations can only be explained by the incorporation of detritus since Th is believed to be insoluble in both the Type I and Type II waters (Figure 2; e.g Langmuir and Herman, 1980; Porcelli and Swarzenski, 2003). Second, light acid leaches (Appendix A) of travertines leave behind significant aluminosilicate detritus, which has higher $U$ and $T h$ concentrations than the carbonate material dissolved during the light acid leach. In contrast, most of the Mg-rich carbonate veins do not leave behind significant aluminosilicate residues after light acid leaches. Third, the $\mathrm{U}$ and Th concentrations of the travertine vein (OM09-84C-MS), which formed in the subsurface and likely incorporated less detritus, are lower than the other travertines. Finally, the travertines have $U$ and Th concentrations which are comparable with highly-altered peridotites and wadi sediments, which are likely sources of detritus.

Since the travertines incorporate significant detritus, constraining the composition of this detritus is important in order to correct for this detrital $\mathrm{Th}$ in the ${ }^{230} \mathrm{Th}$ age calculations (see Section 6.2 below). While fragments of serpentine and highly-altered peridotite are observed in the travertines, these cannot be the only sources of detritus in 
the travertines. This is because the $\mathrm{U}$ and $\mathrm{Th}$ concentrations of the travertine terraces are higher than the $U$ and $T h$ concentrations of the highly-altered peridotites and the serpentine mineral separates.

The two wadi sediments have $\mathrm{U}$ and Th concentrations which are comparable with the $U$ and Th concentrations of the travertine terraces. The wadi sediments contain minerals such as serpentine and enstatite which likely originate from the local peridotite bedrock (Table 1). However, they also contain significant quartz, which is most likely aeolian in origin. The wadi sediments also contain significant carbonate minerals such as calcite, dolomite, and magnesite. The magnesite likely originates from local magnesite veins in peridotite. However, the calcite and dolomite could originate both from local carbonate alteration products in peridotite, such as weathered travertines, and from aeolian fragments from other carbonate deposits which are known to have higher $\mathrm{U}$ concentrations. For example, aeolian carbonate particles could originate from the Cretaceous Natih formation, which has U concentrations up to $\sim 9$ ppm (Fleitmann et al., 2003). The two pseudospeleothems also have lower $U$ and Th concentrations than the travertine terraces. This is likely because the vertical formation and fine-grained texture of the pseudospeleothems reduces the amount of detritus which they incorporate.

\subsection{2: Systematics of $U$ and Th Isotopes:}

The Samail carbonates, peridotites, and sediments either have ${ }^{238} \mathrm{U}$ excesses or fall on the equiline of the $\left({ }^{230} \mathrm{Th} /{ }^{232} \mathrm{Th}\right)$ versus $\left({ }^{238} \mathrm{U} /{ }^{232} \mathrm{Th}\right)$ plot (Figure 6). During initial precipitation from fluids, Samail carbonates should have ${ }^{238} \mathrm{U}$ excesses. Over time, as the ${ }^{238} \mathrm{U}$ decay chain returns to equilibrium, the carbonates will move toward the equiline. 
After approximately 375,000 years ( 5 times the half-life of ${ }^{230} \mathrm{Th}$, which equates to an uncertainty of $3 \%$ in $\left({ }^{230} \mathrm{Th} /{ }^{238} \mathrm{U}\right)$ ), the practical limit of ${ }^{230} \mathrm{Th}$ dating, the carbonate samples will plot on the equiline. Thus, one interpretation is that the carbonate samples which plot on or close to the equiline (five travertines, one $\mathrm{Mg}$-rich carbonate outcrop vein, and four of the Mg-rich carbonate roadcut veins) are 375,000 years or older in age. This interpretation may be valid for carbonate veins, which incorporate lower amounts of aluminosilicate detritus, but may not be valid for surface travertines.

As discussed in Section 6.1.1, the travertines likely contain significant detritus, which is responsible for their elevated $U$ and $T h$ concentrations relative to the subsurface carbonates. Therefore, the travertines which plot near the equiline could either be (1.) 375,000 years or greater in age or (2.) have incorporated a significant quantity of equilibrium (or close to equilibrium) detritus that overwhelms the isotopic signature of the pure travertine. We favor the second explanation for these travertines. The strongest evidence supporting this explanation is the fact that the two recently-formed travertine precipitates (which are actively precipitating from hyperalkaline pools and have $>$ modern ${ }^{14} \mathrm{C}$ compositions, see Chapter 2) fall on the equiline. These precipitates are known to have formed very recently - within the last few years, and perhaps even within days of sampling — so old ${ }^{230} \mathrm{Th}$ ages for these samples can be rejected. Therefore, these samples must have incorporated significant near-equilibrium detritus, such as wadi sediments, which also fall close to the equiline. The isotopic compositions of the other travertines are also likely affected by the incorporation of detritus. 
The fact that the two wadi sediment samples plot close to the equiline is not unexpected. Most aluminosilicate detritus falls on the equiline or has slight ${ }^{230} \mathrm{Th}$ excesses on a $\left({ }^{230} \mathrm{Th} /{ }^{232} \mathrm{Th}\right.$ ) versus $\left({ }^{238} \mathrm{U} /{ }^{232} \mathrm{Th}\right)$ plot (Figure 9). Note that aluminosilicate detritus rarely has ${ }^{238} \mathrm{U}$ excesses (e.g. Szabo and Rosholt, 1982). This is because rocks generally weather under oxidizing conditions present at and near the Earth's surface, where $U$ is soluble and will be leached from rocks.

The ${ }^{238} U$ excesses observed in the highly-altered peridotites (Figures 6 to 8 ) are notable. Since the peridotites formed at $>95 \mathrm{Ma}$, the ${ }^{238} \mathrm{U}$ excesses must be the result of recent alteration or weathering processes that occurred within the last 375,000 years. The most likely explanation is addition of ${ }^{238} \mathrm{U}$ from fluids under reducing conditions, which is consistent with the higher $\mathrm{U}$ concentrations of the highly-altered peridotites relative to less-altered peridotites (Section 6.1.1 and Figure 5). ${ }^{238} \mathrm{U}$ excesses are generally observed in rocks which were deposited at a redox front where U precipitated out of solution. For example, $\mathrm{U}$ concentration enrichments and ${ }^{238} \mathrm{U}$ excesses are commonly observed in places where there has been recent formation or re-mobilization of uranium ore "rollfront" deposits (e.g. Robinson and Rosholt, 1961; Rosholt, 1961 a, b; Dooley et al., 1964, 1966; Rosholt et al., 1964, 1965a, b; Lively et al., 1979). The ${ }^{238} U$ excesses observed in the Samail peridotites are also similar to those observed in sabkha sediments in Syria (Ghaleb et al., 1990) and in the northern Sahara, Algeria (Fontes et al., 1992). At both of these locations, $U$ loss and ${ }^{230} \mathrm{Th}$ excesses were observed in surface sabkha sediments, which are under oxidizing conditions, while $U$ addition and ${ }^{238} \mathrm{U}$ excesses were observed 
in deeper sabkha sediments, which are under reducing conditions (Ghaleb et al., 1990; Fontes et al., 1992).

\subsection{Approaches and Limitations for Determining ${ }^{230} \mathrm{Th}$ Ages for Samail}

\section{Carbonates}

\subsection{1 ${ }^{230}$ Th Dating of Terrestrial Carbonates:}

${ }^{230} \mathrm{Th}$ dating of carbonates measures the ingrowth of ${ }^{230} \mathrm{Th}$ from the decay of ${ }^{238} \mathrm{U}$ and ${ }^{234} \mathrm{U}$ incorporated into the carbonate matrix during formation. Since $\mathrm{Th}$ is highly insoluble relative to $\mathrm{U}$, carbonate that forms (either through inorganic precipitation or biological processes) from marine and terrestrial waters generally contains relatively high $\mathrm{U}$ concentrations and low $\mathrm{Th}$ concentrations. This creates disequilibrium in the ${ }^{238} \mathrm{U}$ decay chain, which gradually returns to equilibrium as ${ }^{238} \mathrm{U}$ (half-life: $\sim 4.47 \times 10^{9}$ years) and ${ }^{234} \mathrm{U}$ (half-life: $\sim 2.45 \times 10^{5}$ years) decay to ${ }^{230}$ Th (half-life: $\sim 7.56 \times 10^{4}$ years). Two intermediate daughters $\left({ }^{234} \mathrm{Th}\right.$ and $\left.{ }^{234} \mathrm{~Pa}\right)$ between ${ }^{238} \mathrm{U}$ and ${ }^{234} \mathrm{U}$ have such short half-lives that they can be ignored and, mathematically, ${ }^{238} \mathrm{U}$ can be treated as if it decays directly to ${ }^{234} \mathrm{U}$. The practical limit of ${ }^{230} \mathrm{Th}$ dating is $\sim 375,000$ years or approximately five times the half-life of ${ }^{230} \mathrm{Th}$.

${ }^{230} \mathrm{Th}$ dating of carbonates relies on two primary assumptions: (1.) there was no initial ${ }^{230} \mathrm{Th}$ incorporated when the carbonate formed, and (2.) there has been no loss or addition of $U$ and $T h$ since carbonate formation. In practice, these two assumptions are only valid for unrecrystallized, pure carbonates such as some corals and dense, pure speleothems. For many other carbonates, these assumptions are not valid and modifications must be made to the dating technique. If initial ${ }^{230} \mathrm{Th}$ is present in a 
carbonate sample, then it may be possible to correct for this initial ${ }^{230} \mathrm{Th}$ (e.g. Bischoff and Fitzpatrick, 1991; Luo and Ku, 1991; Kaufman, 1993; Edwards et al., 2003). Carbonates affected by open-system behavior after formation, such as recrystallized corals and lacustrine carbonates with added hydrogenous Th or $\mathrm{U}$, are challenging to date unless the open system behavior can be estimated using models (e.g. Fontes et al., 1992; Thompson et al., 2003; Villemant and Feullet, 2003; Haase-Schramm et al., 2004).

Terrestrial carbonates are usually affected by the presence of initial ${ }^{230} \mathrm{Th}$ and, to a lesser extent, by ${ }^{234} \mathrm{U}$ and ${ }^{238} \mathrm{U}$ contributed by detrital material (e.g. Bischoff and Fitzpatrick, 1991; Luo and Ku, 1991; Kaufman, 1993). This detritus consists mostly of fine-grained clays but can also contain rock fragments, opaline silica, Fe and $\mathrm{Mn}$ hydroxides, and other materials (e.g. Szabo and Rosholt, 1982). The admixed detritus generally originates from aeolian soil (loess), glacial till, alluvium, colluvium, and other soil (Szabo and Rosholt, 1982; Ku and Liang, 1984; Bischoff and Fitzpatrick, 1991; Luo and $\mathrm{Ku}, 1991)$. Detritus incorporated into terrestrial carbonates is often fine-grained and intergrown with the carbonate, making physical separation very difficult (e.g. Ku and Liang, 1984; Bischoff and Fitzpatrick, 1991; Luo and Ku, 1991).

A variety of approaches have been attempted over the years to account for detrital ${ }^{230} \mathrm{Th},{ }^{234} \mathrm{U}$, and ${ }^{238} \mathrm{U}$ in dirty carbonates. Early studies (e.g. Harmon et al., 1977) attempted to separate the carbonate material from the detrital component by employing light acid leaches. However, numerous subsequent studies (e.g. Schwartz and Latham, 1989; Przybylowicz et al., 1991; Bischoff and Fitzpatrick, 1991; Luo and Ku, 1991) have demonstrated that fractionation of $U$ and Th concentrations and isotopic ratios can occur 
during acid leaching. We also observed fractionation of $U$ and Th concentrations and isotopes during partial dissolution experiments (Appendix A).

A number of studies have investigated and employed pseudoisochron methods for determining the composition of incorporated detritus (e.g. Osmond et al., 1970; Rosholt, 1976; Schwartz and Latham, 1989; Przybylowicz et al., 1991; Bischoff and Fitzpatrick, 1991; Luo and Ku, 1991). However, pseudoisochron methods are limited by the requirement of a set of coeval samples with variable detritus/carbonate ratios which create variable initial $U$ and $T h$ contents. Finding a suitable set of samples with variable amounts of detritus is challenging and not feasible for many geological settings. In addition, pseudoisochron methods which employ partial acid leaches should be approached with caution since they are likely affected by elemental and isotopic fractionation (e.g. Bischoff and Fitzpatrick, 1991; Luo and $\mathrm{Ku}, 1991$ ). The pseudoisochron approach is not appropriate for dating Samail carbonates. For most terrestrial carbonates, ${ }^{230} \mathrm{Th}$ dating techniques employing total sample dissolutions with corrections for detrital ${ }^{230} \mathrm{Th}$ (and sometimes also ${ }^{234} \mathrm{U}$ and ${ }^{238} \mathrm{U}$ ) are preferred (e.g. Ludwig and Titterington, 1994; Edwards et al., 2003; Ludwig, 2003).

The simplest and most commonly employed detrital correction approach uses ${ }^{232} \mathrm{Th}$ as an index for initial ${ }^{230} \mathrm{Th}$ correction. In this method, all ${ }^{232} \mathrm{Th}$ is assumed to originate from detritus, and the measured $\left({ }^{232} \mathrm{Th} /{ }^{238} \mathrm{U}\right)$ of the dirty carbonate is used to weight the ${ }^{230} \mathrm{Th}$ correction in the following equation (e.g. Edwards et al., 2003): 


$$
\left(\frac{{ }^{230} T h}{{ }^{238} U}\right)=1+\left(\left(\frac{{ }^{232} T h}{{ }^{238} U}\right)\left(\frac{{ }^{230} T h}{{ }^{232} T h}\right)_{i}-1\right) e^{-\lambda_{230} t}+\left(\frac{\delta^{234} U_{m}}{1000}\right)\left(\frac{\lambda_{230}}{\lambda_{230}-\lambda_{234}}\right)\left(1-e^{-\left(\lambda_{230}-\lambda_{234}\right) t}\right)
$$

\section{(Equation 1)}

Equation 1 is a modified version of the basic ${ }^{230} \mathrm{Th}^{238} \mathrm{U}$ age equation:

$$
\left(\frac{{ }^{230} T h}{{ }^{238} U}\right)=1-e^{-\lambda_{230} t}+\left(\frac{\delta^{234} U_{m}}{1000}\right)\left(\frac{\lambda_{230}}{\lambda_{230}-\lambda_{234}}\right)\left(1-e^{-\left(\lambda_{230}-\lambda_{234}\right) t}\right)
$$

(Equation 2)

In both equations, $t$ is the age in years, $\lambda_{230}$ is the ${ }^{230} \mathrm{Th}$ decay constant, and $\lambda_{234}$ is the ${ }^{234} \mathrm{U}$ decay constant. For these decay constants, we used the values presented in Cheng et al. (2000). $\left({ }^{230} \mathrm{Th} /{ }^{238} \mathrm{U}\right)$ and $\delta^{234} \mathrm{U}_{\mathrm{m}}$ are calculated from the measured ${ }^{230} \mathrm{Th} /{ }^{238} \mathrm{U}$ and ${ }^{234} \mathrm{U} /{ }^{238} \mathrm{U}$ ratios in the carbonate sample. The additional term in Equation 1 relative to Equation 2 corrects for the presence of initial ${ }^{230} \mathrm{Th}$ from detritus. This term requires two additional values: the $\left({ }^{232} \mathrm{Th} /{ }^{238} \mathrm{U}\right)$ of the carbonate, which can be calculated from the measured $\mathrm{Th}$ and $\mathrm{U}$ concentrations, and a term called $\left({ }^{230} \mathrm{Th} /{ }^{232} \mathrm{Th}\right)_{\mathrm{i}}$, which is the assumed $\left({ }^{230} \mathrm{Th} /{ }^{232} \mathrm{Th}\right)$ composition of the contaminating detritus.

The $\left({ }^{230} \mathrm{Th} /{ }^{232} \mathrm{Th}\right)$ composition of the detrital material is rarely known explicitly and is generally the largest source of uncertainty in corrected ${ }^{230} \mathrm{Th}$ ages. Often, a Bulk Silicate Earth $\left({ }^{230} \mathrm{Th} /{ }^{232} \mathrm{Th}\right)$ value of 0.814 (calculated assuming equilibrium with $\left.\left({ }^{238} \mathrm{U} /{ }^{232} \mathrm{Th}\right)=\left({ }^{230} \mathrm{Th} /{ }^{232} \mathrm{Th}\right)\right)$ is assumed for the detritus. This first order estimation is adequate for older samples with high $\mathrm{U}$ and low ${ }^{232} \mathrm{Th}$ contents as the correction is small, and therefore the assumed $\left({ }^{230} \mathrm{Th} /{ }^{232} \mathrm{Th}\right)$ value of the detritus can vary greatly without having a large effect on the calculated age (e.g. Bischoff and Fitzpatrick, 1991; Kaufman, 1993; Ludwig and Titterington, 1994). However, for young samples and also samples 
with relatively low $U$ contents and high ${ }^{232} \mathrm{Th}$ contents, such as the Samail carbonates, the assumed isotopic values for the detritus have a large effect on the calculated age, and therefore these values must be known explicitly in order to determine reliable corrected ${ }^{230}$ Th ages.

To evaluate a reasonable range of detrital $\left({ }^{230} \mathrm{Th} /{ }^{232} \mathrm{Th}\right)$ values to use for the initial ${ }^{230} \mathrm{Th}$ correction for Samail carbonates, we compiled 300 literature values for $\left({ }^{230} \mathrm{Th} /{ }^{232} \mathrm{Th}\right)$ measured in various types of aluminosilicate detritus (see Figure 9 and Section 6.1.2 above). These 300 literature analyses have $\left({ }^{230} \mathrm{Th} /{ }^{232} \mathrm{Th}\right)$ ranging from 0.02 4.61 (average: $0.77 ; 2 \sigma$ standard deviation: 0.50 ). While the average value of the detrital compilation is fairly close to the equilibrium Bulk Silicate Earth value of 0.814 , detritus clearly has a much wider range of $\left({ }^{230} \mathrm{Th} /{ }^{232} \mathrm{Th}\right)$ values. Furthermore, the $\left({ }^{230} \mathrm{Th} /{ }^{232} \mathrm{Th}\right)$ value of detritus incorporated into Samail carbonates is likely higher than both average detritus and equilibrium Bulk Silicate Earth values. The recently-formed travertine precipitates OM09-8COPS-MS and OM09-10COPS-MS have $\left({ }^{230} \mathrm{Th} /{ }^{232} \mathrm{Th}\right)$ values of 1.376 and 1.416 , respectively (Figure 8 ). These two precipitates are close to zero-age (based on ${ }^{14} \mathrm{C}$ measurements and field observations, see Chapter 2), and therefore these $\left({ }^{230} \mathrm{Th} /{ }^{232} \mathrm{Th}\right)$ measurements reflect the values of detritus being incorporated into travertines forming today. While the $\left({ }^{230} \mathrm{Th} /{ }^{232} \mathrm{Th}\right)$ value of detritus incorporated into Samail carbonates may be spatially and temporally variable, the $\left({ }^{230} \mathrm{Th} /{ }^{232} \mathrm{Th}\right)$ values measured in the recently-formed travertine precipitates are similar and provide a possible correction value to use for older travertine samples. 
$\left({ }^{230} \mathrm{Th} /{ }^{232} \mathrm{Th}\right)$ measurements in wadi sediments and highly-altered peridotites also provide detrital values to use for initial ${ }^{230} \mathrm{Th}$ corrections. Three of the highly-altered peridotites and one of the wadi sediments have $\left({ }^{230} \mathrm{Th} /{ }^{232} \mathrm{Th}\right)$ values of $\sim 1.4-1.6$, which are similar to the values measured in the recently-formed travertine precipitates. However, wadi sediment OM09-8S-MS, which consists primarily of serpentine, has higher $\left({ }^{230} \mathrm{Th} /{ }^{232} \mathrm{Th}\right)$ of 5.215 , and the highly-altered peridotite OM10-13C-MG has even higher $\left({ }^{230} \mathrm{Th} /{ }^{232} \mathrm{Th}\right)$ of 12.367 . The higher values of these samples may reflect ingrowth of ${ }^{230} \mathrm{Th}$ from ${ }^{238} \mathrm{U}$ and ${ }^{234} \mathrm{U}$ that was added during serpentinization. Unfortunately, there is not a tight range of $\left({ }^{230} \mathrm{Th} /{ }^{232} \mathrm{Th}\right)$ values to use for correction of the Samail carbonate ages for initial ${ }^{230} \mathrm{Th}$.

We have employed total dissolutions with corrections for the contribution of initial ${ }^{230} \mathrm{Th}$ in an attempt to determine ${ }^{230} \mathrm{Th}$ ages for Samail carbonates. We have not employed corrections for detrital ${ }^{234} \mathrm{U}$ and ${ }^{238} \mathrm{U}$ because (1.) the initial ${ }^{230} \mathrm{Th}$ correction has the largest influence on the age corrections, and (2.) corrections for detrital ${ }^{234} \mathrm{U}$ and ${ }^{238} \mathrm{U}$ in addition to ${ }^{230} \mathrm{Th}$ are not practical because of the heterogeneous composition of possible detritus incorporated into the Samail carbonates. Because Samail carbonates are so sensitive to initial ${ }^{230} \mathrm{Th}$ corrections and also there is not an obvious single detrital $\left({ }^{230} \mathrm{Th} /{ }^{232} \mathrm{Th}\right)$ value to use for the corrections, a sensitivity analysis approach has been employed below to evaluate the influence of detrital ${ }^{230} \mathrm{Th}$ on ${ }^{230} \mathrm{Th}$ ages of Samail carbonates. 


\subsection{2: ${ }^{230}$ Th Dating of Travertines:}

Uncorrected ${ }^{230} \mathrm{Th}$ ages for Samail travertines are presented in Table 3. The uncorrected ages were calculated using Equation 2 with no correction for initial ${ }^{230} \mathrm{Th}$ from detritus. For all travertines, the uncorrected ${ }^{230} \mathrm{Th}$ ages are significantly older than the corresponding ${ }^{14} \mathrm{C}$ ages, which are presented in Chapter 2.

The low $\mathrm{U}$ concentrations and relatively high $\mathrm{Th} / \mathrm{U}$ ratios found in Samail travertines make obtaining detritus-corrected ${ }^{230} \mathrm{Th}$ ages highly challenging. The travertines contain significant detrital $\mathrm{Th}$, and the values of corrected ${ }^{230} \mathrm{Th}$ ages are highly sensitive to the selected value of initial $\left({ }^{230} \mathrm{Th} /{ }^{232} \mathrm{Th}\right)$ used in the correction. For all travertine samples, varying the assumed initial $\left({ }^{230} \mathrm{Th} /{ }^{232} \mathrm{Th}\right)$ over the typical range observed in aluminosilicate detritus changes the ${ }^{230} \mathrm{Th}$ age by tens of thousands of years. As an example, Figure 10 displays the effect of the selected initial $\left({ }^{230} \mathrm{Th} /{ }^{232} \mathrm{Th}\right)$ on the corrected ${ }^{230} \mathrm{Th}$ age for travertine terrace sample OM09-106C-MS-A. Similar diagrams for all travertine samples are presented in Appendix B. Depending on the selected detrital value of $\left({ }^{230} \mathrm{Th} /{ }^{232} \mathrm{Th}\right)$, the ${ }^{230} \mathrm{Th}$-corrected age of OM09-106C-MS-A ranges from approximately 50,000 years to 0 years. However, an assumed detrital $\left({ }^{230} \mathrm{Th} /{ }^{232} \mathrm{Th}\right)$ value of approximately 1.7 , which is slightly higher than the values of $\sim 1.3-1.6$ measured in the recently-formed travertine precipitates and three of the highly-altered peridotites, produces a corrected ${ }^{230} \mathrm{Th}$ age which is concordant with the ${ }^{14} \mathrm{C}$ age for this sample of $30,023 \pm 235$ yr BP (see Chapter 2).

For all travertine samples, geologically plausible detrital $\left({ }^{230} \mathrm{Th} /{ }^{232} \mathrm{Th}\right)$ values bring the corrected ${ }^{230} \mathrm{Th}$ ages into concordance with the corresponding ${ }^{14} \mathrm{C}$ ages. 
Unfortunately, no single detrital $\left({ }^{230} \mathrm{Th} /{ }^{232} \mathrm{Th}\right)$ value corrects the travertine ${ }^{230} \mathrm{Th}$ ages to the corresponding ${ }^{14} \mathrm{C}$ ages. $\left({ }^{230} \mathrm{Th} /{ }^{232} \mathrm{Th}\right)$ values of approximately 0.8 and 1.5 bring the corrected ${ }^{230}$ Th ages of travertine terrace samples OM09-106C-MS-B and OM09-109CMS, respectively, into concordance with the ${ }^{14} \mathrm{C}$ ages. Similarly, a $\left({ }^{230} \mathrm{Th} /{ }^{232} \mathrm{Th}\right)$ value just above 1 brings the corrected ${ }^{230} \mathrm{Th}$ ages of the two samples from the travertine pseudospeleothem into concordance with the ${ }^{14} \mathrm{C}$ ages. However, higher $\left({ }^{230} \mathrm{Th} /{ }^{232} \mathrm{Th}\right)$ values of approximately 4 and 5.2 are required to bring the corrected ${ }^{230} \mathrm{Th}$ ages of travertine terrace sample OM09-107C-MS and travertine vein OM09-84C-MS, respectively, into concordance with the ${ }^{14} \mathrm{C}$ ages. These two $\left({ }^{230} \mathrm{Th} /{ }^{232} \mathrm{Th}\right)$ values are outside of the typical range of aluminosilicate detritus. However, these correction values are between those values typically observed in aluminosilicate detritus and those observed in the serpentine-rich wadi sediment OM09-8S-MS $\left(\left(\left(^{230} \mathrm{Th} /{ }^{232} \mathrm{Th}\right)=5.215\right)\right.$, and in the highly-altered peridotite OM10-13C-MS $\left(\left({ }^{230} \mathrm{Th} /{ }^{232} \mathrm{Th}\right)=12.367\right)$.

The different $\left({ }^{230} \mathrm{Th} /{ }^{232} \mathrm{Th}\right)$ correction values required for each travertine are likely a result of the heterogeneous nature of the detritus, which covers a wide range of $\left({ }^{230} \mathrm{Th} /{ }^{232} \mathrm{Th}\right)$ values (Section 6.2 .1 and Figure 8$)$. Therefore, although geologically plausible detrital $\left({ }^{230} \mathrm{Th} /{ }^{232} \mathrm{Th}\right)$ values can be selected for each travertine to bring the corrected ${ }^{230} \mathrm{Th}$ age into concordance with the ${ }^{14} \mathrm{C}$ age, the lack of a uniform $\left({ }^{230} \mathrm{Th} /{ }^{232} \mathrm{Th}\right)$ correction value for all travertines precludes obtaining precise ages for travertines using ${ }^{230}$ Th dating alone. 


\subsection{3 ${ }^{230}$ Th Dating of Mg-Rich Carbonate Veins:}

Uncorrected ${ }^{230} \mathrm{Th}$ ages (with no correction for initial ${ }^{230} \mathrm{Th}$ from detritus) for Mgrich carbonate veins are presented in Table 3.

Four out of the five dated Mg-rich carbonate outcrop veins have uncorrected ${ }^{230} \mathrm{Th}$ ages which are younger than the corresponding ${ }^{14} \mathrm{C}$ ages. The exception is outcrop vein OM10-84C-MG, which has a ${ }^{230} \mathrm{Th}$ age older than the corresponding ${ }^{14} \mathrm{C}$ age. While not particularly high, the $\sim 2 \mathrm{ppb}$ Th concentration of sample OM10-84C-MG is significant relative to its low $\mathrm{U}$ concentration of $\sim 1 \mathrm{ppb}$. Indeed, the $\mathrm{Th} / \mathrm{U}$ ratio of $\mathrm{OM} 10-84 \mathrm{C}-\mathrm{MG}$ is significantly higher than the other carbonate outcrop veins, which have $\mathrm{Th} / \mathrm{U}$ ratios of $\sim 0.2-0.3$. Therefore, OM10-84C-MG likely contains significant initial ${ }^{230} \mathrm{Th}$ relative to its U concentration.

Since the uncorrected ${ }^{230} \mathrm{Th}$ ages for carbonate outcrop veins are generally younger than the ${ }^{14} \mathrm{C}$ ages, corrections for initial ${ }^{230} \mathrm{Th}$ from detritus cannot be employed to make the two sets of ages concordant since initial ${ }^{230} \mathrm{Th}$ corrections will make the ${ }^{230} \mathrm{Th}$ ages even younger. This is illustrated for outcrop vein OM10-13C-MG (Figure 11) and in similar figures for the other carbonate outcrop veins (Appendix B). Possibly, a detrital correction employing an unusual detritus with ${ }^{238} \mathrm{U}$ enrichment, such as that observed in the altered peridotites, could be employed to make the ${ }^{230} \mathrm{Th}$ ages older. However, this is challenging to do because the carbonate outcrop veins and the altered peridotites have very similar $\left({ }^{234} \mathrm{U}^{238} \mathrm{U}\right)$ compositions (Figure 7).

There are two possible explanations for the discrepancy between the ${ }^{14} \mathrm{C}$ ages and

${ }^{230} \mathrm{Th}$ ages for the carbonate outcrop veins. The first explanation is that the ${ }^{230} \mathrm{Th}$ ages are 
valid, but the ${ }^{14} \mathrm{C}$ ages are too old because of the incorporation of recycled ${ }^{14} \mathrm{C}$ dead carbon. The second explanation is that the ${ }^{230} \mathrm{Th}$ ages are too young because of ${ }^{238} \mathrm{U}$ addition from U-bearing fluids. While both explanations are feasible, we prefer the second explanation because of the ${ }^{238} \mathrm{U}$ excesses observed in the altered peridotites. Furthermore, the similar $\left({ }^{234} \mathrm{U}{ }^{238} \mathrm{U}\right.$ ) values of the highly-altered peridotites (except for the anomalous sample OM10-1P-MG) and the carbonate outcrop veins suggest that they may have been altered by similar fluids. If the carbonate outcrop veins were modified by $\mathrm{U}$ addition from fluid, then their ${ }^{14} \mathrm{C}$ ages may have also been partially or fully re-set. Therefore, ${ }^{14} \mathrm{C}$ ages of altered $\mathrm{Mg}$-rich carbonate veins should be interpreted with caution and likely represent minimum ages.

In comparison to the carbonate outcrop veins, the uncorrected ${ }^{230} \mathrm{Th}$ ages for the carbonate roadcut veins are significantly older with $\left({ }^{230} \mathrm{Th} /{ }^{238} \mathrm{U}\right)$ approaching equilibrium values. Roadcut veins OM09-35C-MS, OM09-55C-MS, and OM09-57C-MS have $\left({ }^{230} \mathrm{Th} /{ }^{238} \mathrm{U}\right)$ values of $1.068,0.956$, and 0.984 , respectively. Furthermore, veins OM0955C-MS, OM09-57C-MS, and OM09-58C-MS have $\left({ }^{234} \mathrm{U} /{ }^{238} \mathrm{U}\right)$ values of $1.044,1.010$, and 0.998 , respectively, which also approach equilibrium values. Unfortunately, we could not obtain a $\mathrm{U}$ isotopic measurement for OM09-35C-MS or a Th isotopic measurement for OM09-58C-MS.

The ${ }^{14} \mathrm{C}$ ages for these four roadcut veins are $>50,000$ years $\mathrm{BP}\left({ }^{14} \mathrm{C}\right.$ dead, see Chapter 2), so the older ${ }^{230} \mathrm{Th}$ ages suggested by the near-equilibrium $\left({ }^{230} \mathrm{Th} /{ }^{238} \mathrm{U}\right)$ and $\left({ }^{234} \mathrm{U} /{ }^{238} \mathrm{U}\right)$ are consistent with the ${ }^{14} \mathrm{C}$ dating. As shown in Figure 12, applying a wide range of $\left({ }^{230} \mathrm{Th} /{ }^{232} \mathrm{Th}\right)$ detrital values for an initial ${ }^{230} \mathrm{Th}$ correction does not affect the 
${ }^{230} \mathrm{Th}$ age of OM09-57C-MS significantly (i.e. the sample remains greater than 350,000 years in age). However, corrections for initial ${ }^{230} \mathrm{Th}$ do affect the ${ }^{230} \mathrm{Th}$ age for OM0955C-MS (Figure 13), which has a higher $\mathrm{Th} / \mathrm{U}$ ratio than OM09-57C-MS. However, the near-equilibrium $\left({ }^{234} \mathrm{U} /{ }^{238} \mathrm{U}\right)$ values for these two veins suggest that they are greater than 375,000 years in age, and may be significantly older since it takes much longer (over 1 million years, due to the longer half-life of $\left.{ }^{234} U\right)$ for $\left({ }^{234} U /{ }^{238} U\right)$ to return to equilibrium.

Roadcut vein OM10-52C-MG has a $\left({ }^{230} \mathrm{Th} /{ }^{238} \mathrm{U}\right)$ value of 1.542 , which is a value that cannot be reached through closed-system decay and ingrowth and which is outside of the valid range for the ${ }^{230} \mathrm{Th}$ age equation. Therefore, no ${ }^{230} \mathrm{Th}$ ages can be determined for OM10-52C-MG. The high $\left({ }^{230} \mathrm{Th} /{ }^{238} \mathrm{U}\right)$ value observed for OM10-52C-MG is likely explained by the influence of detrital ${ }^{230} \mathrm{Th}$ since this sample has a very high $\mathrm{Th} / \mathrm{U}$ ratio of 5.6.

\section{3: Implications for Natural Rates of Carbonate Formation in the Peridotite}

\section{Layer of the Samail Ophiolite:}

The determination of precise ${ }^{230} \mathrm{Th}$ ages for Samail carbonates is limited by the large and uncertain initial ${ }^{230} \mathrm{Th}$ corrections. Nevertheless, age limits from ${ }^{230} \mathrm{Th}$ dating of Samail carbonates as well as the $\mathrm{U}$ and $\mathrm{Th}$ systematics for Samail carbonates and peridotites permit further refinement of estimates of natural rates of $\mathrm{CO}_{2}$ sequestration in Samail travertines and subsurface carbonate veins that were presented in Chapter 2 and which were first estimated by Kelemen and Matter (2008).

The fact that geologically reasonable initial ${ }^{230} \mathrm{Th}$ corrections bring ${ }^{230} \mathrm{Th}$ and ${ }^{14} \mathrm{C}$ travertine ages into concordance provides further support that ${ }^{14} \mathrm{C}$ dating of travertines is 
generally reliable (with the exception of some highly-weathered, porous travertines; see details in Chapter 2) and that these ages can be interpreted as formation ages. This places additional confidence in the estimates based on ${ }^{14} \mathrm{C}$ dating (see Chapter 2) that travertine precipitation rates are $\sim 0.1-0.3 \mathrm{~mm} / \mathrm{yr}$ and that travertines are sequestering a maximum $\sim 1-3 \times 10^{6} \mathrm{~kg} \mathrm{CO} / \mathrm{yr}$.

$\mathrm{U}$ and Th systematics and ${ }^{230} \mathrm{Th}$ ages also provide further evidence that there are multiple generations of formation of $\mathrm{Mg}$-rich carbonate veins in the peridotite subsurface. The ${ }^{238} \mathrm{U}$ excesses observed in highly-altered peridotites indicate that alteration of these peridotites is a fairly recent process since these excesses must have been created within the past 375,000 years as otherwise the $\left({ }^{230} \mathrm{Th} /{ }^{238} \mathrm{U}\right)$ values would be in equilibrium. Therefore, serpentinization of Samail peridotites appears to be an ongoing process that is not limited to older alteration-for example, alteration which occurred on the seafloor or during obduction of the ophiolite $>80 \mathrm{Ma}$. The fact that the Mg-rich carbonate outcrop veins have ${ }^{230} \mathrm{Th}$ ages younger than the corresponding ${ }^{14} \mathrm{C}$ ages suggests that these carbonate veins may have been altered by U-bearing carbonate fluids, a process which also must have occurred within the last 375,000 years. The lack of concordance between ${ }^{230} \mathrm{Th}$ and ${ }^{14} \mathrm{C}$ ages for the outcrop veins also indicates that veins are affected by opensystem behavior, most likely dissolution and re-precipitation of previous generations of carbonate veins. Such open-system behavior obscures the range of ages for Mg-rich carbonate veins.

The near-equilibrium $\left({ }^{230} \mathrm{Th}-{ }^{238} \mathrm{U}\right)$ and $\left({ }^{234} \mathrm{U} /{ }^{238} \mathrm{U}\right)$ values measured in lessweathered, ${ }^{14} \mathrm{C}$ dead $\mathrm{Mg}$-rich carbonate veins sampled at roadcuts indicate that, at least at 
certain exposures such as Fanja Roadcut, there are much older $(>1$ million years for at least two veins) generations of $\mathrm{Mg}$-rich carbonate veins preserved. Therefore, carbonate vein formation in the peridotite layer of the Samail ophiolite has likely been ongoing for at least the past 1 million years and possibly for a much longer time. Hence, sensitivity analysis - such as that presented in Chapter 2- that considers the impact of multiple generations of vein formation on the estimated rate of $\mathrm{CO}_{2}$ sequestration in subsurface carbonate veins is essential since there is no single "average" carbonate vein age. The sensitivity analysis presented in Chapter 2 indicates that ongoing Mg-rich carbonate vein formation in the peridotite layer of the Samail Ophiolite sequesters on the order of $10^{7} \mathrm{~kg}$ $\mathrm{CO}_{2} / \mathrm{yr}$.

\section{Summary:}

Samail carbonates have extremely low $\mathrm{U}$ and $\mathrm{Th}$ concentrations which reflect the low $\mathrm{U}$ and $\mathrm{Th}$ concentrations found in Samail peridotites and associated groundwaters. The higher $U$ and $T h$ concentrations observed in surface travertine terraces relative to subsurface carbonate veins likely originate from the higher proportion of aluminosilicate detritus incorporated into these surface samples. Highly-altered Samail peridotites have

${ }^{238} \mathrm{U}$ excesses and higher $\mathrm{U}$ concentrations than less-altered Samail peridotites. The ${ }^{238} \mathrm{U}$ excesses and elevated $U$ concentrations are likely a result of fluid addition of $U$ under reducing conditions during recent (within the last 375,000 years) serpentinization in the subsurface.

The low $U$ concentrations and relatively high $\mathrm{Th} / \mathrm{U}$ ratios found in Samail travertines make obtaining ${ }^{230} \mathrm{Th}$ ages highly challenging. The travertines contain 
significant initial ${ }^{230} \mathrm{Th}$ from detritus, and the values of ages obtained through correction for ${ }^{230} \mathrm{Th}$ from detritus are highly sensitive to the selected value of initial $\left({ }^{230} \mathrm{Th} /{ }^{232} \mathrm{Th}\right)$ used in the correction. For all travertine samples, varying the initial $\left({ }^{230} \mathrm{Th} /{ }^{232} \mathrm{Th}\right)$ used in the correction changes the age of the travertine by tens of thousands of years. However, geologically plausible initial $\left({ }^{230} \mathrm{Th} /{ }^{238} \mathrm{U}\right)$ values correct the travertine ${ }^{230} \mathrm{Th}$ ages to the corresponding ${ }^{14} \mathrm{C}$ ages. Mg-rich carbonate outcrop veins have uncorrected ${ }^{230} \mathrm{Th}$ ages which are younger than the corresponding ${ }^{14} \mathrm{C}$ ages. The most likely explanation for this is addition of ${ }^{238} \mathrm{U}$ from fluids that makes the ${ }^{230} \mathrm{Th}$ ages too young. ${ }^{14} \mathrm{C}$ dead, $\mathrm{Mg}$-rich carbonate roadcut veins have $\left({ }^{230} \mathrm{Th} /{ }^{238} \mathrm{U}\right)$ and $\left({ }^{234} \mathrm{U} /{ }^{238} \mathrm{U}\right)$ values approaching equilibrium and are likely $>375,000$ years in age.

$\mathrm{U}$ and Th systematics and ${ }^{230} \mathrm{Th}$ age limits support the conclusions of ${ }^{14} \mathrm{C}$ dating (see Chapter 2; also Clark and Fontes, 1990; Kelemen and Matter, 2008, and Kelemen et al., 2011) that carbonate formation in the Samail Ophiolite is an ongoing process. These new data also support the interpretation that there are multiple generations of subsurface carbonate vein formation that must be considered when estimating natural rates of $\mathrm{CO}_{2}$ sequestration in the peridotite layer of the Samail Ophiolite.

\section{Acknowledgments:}

This research was done in collaboration with my supervisors, Kenneth Sims, Susan Humphris, and Peter Kelemen. Ali Al-Rajhi, Ministry of Commerce and Industry, Sultanate of Oman, and Sobhi Nasir, Sultan Qaboos University, are thanked for logistical support during field seasons. Jürg Matter, Everett Shock, Lisa Streit, Amelia Paukert, and Ernest Gauntlett, and others are thanked for assistance with Oman fieldwork and for 
scientific discussions. Jerzy Blustajn and Margaret Sulanowska are thanked for assistance with sample preparation and laboratory work. Jürg Matter and Everett Shock are thanked for providing the water samples and for sharing the results of ICP-MS $U$ and Th measurements on Samail waters. Vera Pavel is thanked for assistance with MATLAB programming. Marguerite Goddard is thanked for providing additional information about the $U$ and Th analyses presented in Hanghøj et al. (2010). This research was funded by National Science Foundation grant EAR-1049281, the Deep Ocean Exploration Institute at Woods Hole Oceanographic Institution, the Academic Programs Office at Woods Hole Oceanographic Institution, the Mellon Foundation, and a Research Initiative in Science and Engineering Grant from Columbia University to Peter Kelemen and Jürg Matter and also Kelemen's Charles Francis Adams Chair at Columbia University. 
Tables:

Table 1: Sample Locations and Descriptions

\begin{tabular}{|c|c|c|c|c|c|c|}
\hline Sample Name: & Location: & $\begin{array}{c}\text { UTM- } \\
\text { Easting: }\end{array}$ & $\begin{array}{c}\text { UTM- } \\
\text { Northing: }\end{array}$ & Description: & $\begin{array}{l}\text { Major Minerals } \\
\text { (XRD): } \\
\end{array}$ & Trace Minerals (XRD): \\
\hline \multicolumn{7}{|l|}{ Travertines: } \\
\hline $\begin{array}{c}\text { OM09-76C-MS-A } \\
\text { (Top) }\end{array}$ & $\begin{array}{l}\text { Wadi Sudari } \\
\text { Travertine }\end{array}$ & 0443115 & 2650257 & $\begin{array}{c}\text { Layered } \\
\text { pseudospeleothem } \\
\text { (drop) }\end{array}$ & calcite & none \\
\hline $\begin{array}{l}\text { OM09-76C-MS-C } \\
\text { (Bottom) }\end{array}$ & $\begin{array}{l}\text { Wadi Sudari } \\
\text { Travertine } \\
\end{array}$ & 0443115 & 2650257 & $\begin{array}{c}\text { Layered } \\
\text { pseudospeleothem } \\
\text { (drop) }\end{array}$ & calcite & unidentified clay \\
\hline OM09-84C-MS & $\begin{array}{c}\text { Wadi Sudari } \\
\text { Travertine } \\
\end{array}$ & 0443082 & 2650304 & Travertine vein & calcite, brucite & $\begin{array}{l}\text { hydromagnesite?, } \\
\text { unidentified clay }\end{array}$ \\
\hline OM09-8COPS-MS & $\begin{array}{c}\text { Wadi Sudari } \\
\text { Travertine }\end{array}$ & 0443118 & 2650078 & $\begin{array}{l}\text { Recently-formed } \\
\text { travertine precipitate }\end{array}$ & calcite & $\begin{array}{c}\text { aragonite, } \\
\text { hydromagnesite?, } \\
\text { unidentified clay }\end{array}$ \\
\hline $\begin{array}{l}\text { OM09-106C-MS-A } \\
\text { (Top) } \\
\end{array}$ & $\begin{array}{c}\text { Wadi } \\
\text { Uqaybah } \\
\text { Travertine } \\
\end{array}$ & 0426245 & 2633924 & Travertine terrace & calcite & none \\
\hline $\begin{array}{l}\text { OM09-106C-MS-B } \\
\text { (Bottom) }\end{array}$ & $\begin{array}{c}\text { Wadi } \\
\text { Uqaybah } \\
\text { Travertine } \\
\end{array}$ & 0426245 & 2633924 & Travertine terrace & calcite & unidentified clay \\
\hline OM09-107C-MS & $\begin{array}{c}\text { Wadi } \\
\text { Uqaybah } \\
\text { Travertine } \\
\end{array}$ & 0426309 & 2633950 & Travertine terrace & calcite & unidentified clay \\
\hline OM09-109C-MS & $\begin{array}{c}\text { Wadi } \\
\text { Uqaybah } \\
\text { Travertine } \\
\end{array}$ & 0426208 & 2633925 & Travertine terrace & calcite & none \\
\hline OM09-10COPS-MS & $\begin{array}{c}\text { Wadi } \\
\text { Uqaybah } \\
\text { Travertine } \\
\end{array}$ & 0426183 & 2633965 & $\begin{array}{l}\text { Recently-formed } \\
\text { travertine precipitate }\end{array}$ & calcite & quartz, unidentified clay \\
\hline
\end{tabular}




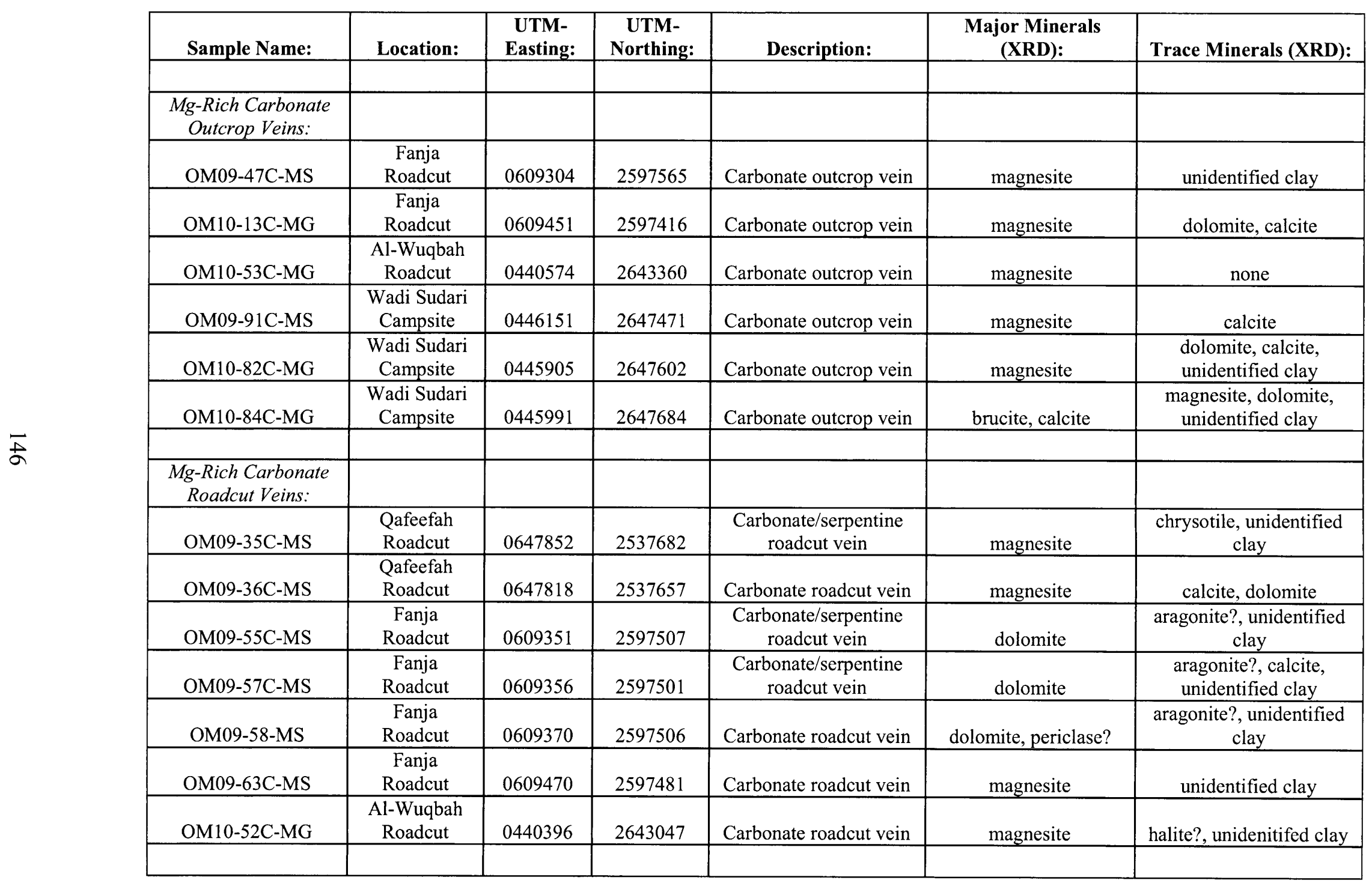




\begin{tabular}{|c|c|c|c|c|c|c|}
\hline Sample Name: & Location: & $\begin{array}{c}\text { UTM- } \\
\text { Easting: }\end{array}$ & $\begin{array}{c}\text { UTM- } \\
\text { Northing: }\end{array}$ & Description: & $\begin{array}{l}\text { Major Minerals } \\
\text { (XRD): }\end{array}$ & Trace Minerals (XRD): \\
\hline \multicolumn{7}{|l|}{ Peridotites: } \\
\hline OM10-1P-MG & $\begin{array}{c}\text { Fanja } \\
\text { Roadcut }\end{array}$ & 0440396 & 2643047 & Highly-altered dunite & lizardite & magnesite \\
\hline OM10-2P-MG & $\begin{array}{c}\text { Fanja } \\
\text { Roadcut }\end{array}$ & 0609451 & 2597416 & Highly-altered dunite & chrysotile, forsterite & magnesite, hydrotalcite \\
\hline OM10-13P-MG & $\begin{array}{c}\text { Misht } \\
\text { Travertine }\end{array}$ & 0487305 & 2576134 & $\begin{array}{c}\text { Highly-altered } \\
\text { harzburgite }\end{array}$ & chrysotile, calcite & forsterite, spinel \\
\hline OM10-17P-MG & $\begin{array}{c}\text { Misht } \\
\text { Travertine }\end{array}$ & 0489565 & 2575412 & $\begin{array}{c}\text { Highly-altered } \\
\text { harzburgite }\end{array}$ & chrysotile, forsterite & enstatite, spinel \\
\hline OM10-19P-MG & $\begin{array}{l}\text { Wadi Sudari } \\
\text { Travertine }\end{array}$ & 0443154 & 2650213 & $\begin{array}{c}\text { Highly-altered } \\
\text { harzburgite }\end{array}$ & Lizardite & spinel \\
\hline OM10-22P-MG & $\begin{array}{l}\text { Wadi Sudari } \\
\text { Campsite }\end{array}$ & 0445924 & 2647613 & $\begin{array}{c}\text { Highly-altered } \\
\text { harzburgite }\end{array}$ & $\begin{array}{c}\text { chrysotile, forsterite, } \\
\text { enstatite }\end{array}$ & diopside, spinel \\
\hline OM94-74h & $\begin{array}{c}\text { Wadi Tayin } \\
\text { Massif }\end{array}$ & 0640617 & 2548967 & Harzburgite & 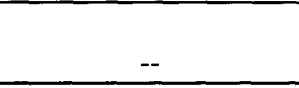 & -- \\
\hline OM94-74d & $\begin{array}{c}\text { Wadi Tayin } \\
\text { Massif }\end{array}$ & 0640617 & 2548967 & Dunite & - & -- \\
\hline OM94-104 & $\begin{array}{c}\text { Wadi Tayin } \\
\text { Massif } \\
\end{array}$ & 0641147 & 2536242 & Harzburgite & -- & -- \\
\hline \multicolumn{7}{|l|}{ Serpentines: } \\
\hline $\begin{array}{l}\text { OM10-27C-MG- } \\
\text { SERP }\end{array}$ & $\begin{array}{l}\text { Qafeefah } \\
\text { Roadcut }\end{array}$ & 0647791 & 2537644 & $\begin{array}{l}\text { Carbonate/serpentine } \\
\text { roadcut vein }\end{array}$ & Lizardite & none \\
\hline $\begin{array}{l}\text { OM10-54C-MG- } \\
\text { SERP }\end{array}$ & $\begin{array}{l}\text { Al-Wuqbah } \\
\text { Roadcut }\end{array}$ & 0440832 & 2643931 & $\begin{array}{c}\text { Carbonate/serpentine } \\
\text { roadcut vein }\end{array}$ & Lizardite & none \\
\hline \multicolumn{7}{|l|}{ Sediments: } \\
\hline OM09-8S-MS & $\begin{array}{c}\text { Fanja } \\
\text { Roadcut }\end{array}$ & 0609493 & 2597536 & $\begin{array}{l}\text { Sediment collected } \\
\text { from wadi floor }\end{array}$ & Lizardite & $\begin{array}{l}\text { quartz, dolomite, } \\
\text { magnesite, calcite }\end{array}$ \\
\hline OM09-12S-MS & $\begin{array}{c}\text { Wadi } \\
\text { Uqaybah } \\
\text { Travertine }\end{array}$ & 0426173 & 2633970 & $\begin{array}{l}\text { Sediment collected } \\
\text { from wadi floor }\end{array}$ & quartz, calcite & $\begin{array}{l}\text { chrysotile, dolomite, } \\
\text { enstatite, aragonite? }\end{array}$ \\
\hline
\end{tabular}




\begin{tabular}{|c|c|c|c|c|c|c|}
\hline Sample Name: & Location: & $\begin{array}{c}\text { UTM- } \\
\text { Easting: }\end{array}$ & $\begin{array}{c}\text { UTM- } \\
\text { Northing: }\end{array}$ & Description: & $\begin{array}{c}\text { Major Minerals } \\
\text { (XRD): }\end{array}$ & Trace Minerals (XRD): \\
\hline Silicate Standards: & & & & & & \\
\hline ATHO & Iceland & -- & -- & Rhyolite & -- & - \\
\hline BHVO-1 & Hawaii & -- & -- & Basalt & -- & - \\
\hline PCC-1 & California & -- & -- & Peridotite & -- & - \\
\hline DTS-1 & $\begin{array}{c}\text { Washington } \\
\text { (state) }\end{array}$ & -- & & Dunite & -- & \\
\hline Carbonate Standard: & & & & & & \\
\hline JCp-1 & $\begin{array}{c}\text { Ryukyu } \\
\text { Islands, Japan }\end{array}$ & -- & -- & Porites coral & -- & \\
\hline
\end{tabular}

Major and trace mineral identifications were determined on powdered samples by X-ray Diffraction at Woods Hole

Oceanographic Institution. The minerals were identified using MacDiff, Version 4.2. 
Table 2: $\mathrm{U}$ and $\mathrm{Th}$ Concentrations

\begin{tabular}{|c|c|c|c|c|c|}
\hline Sample Name: & $\mathrm{U}(\mathrm{ppm})$ & 2 s.d. (\%): & Th (ppm): & 2 s.d. (\%): & Th/U: \\
\hline \multicolumn{6}{|l|}{ Travertines: } \\
\hline OM09-76C-MS-A (Top) & 0.0447 & 1.40 & 0.0673 & 1.20 & 1.5070 \\
\hline OM09-76C-MS-C (Bottom) & 0.0195 & 1.40 & 0.0472 & 1.20 & 2.4239 \\
\hline OM09-8COPS-MS & 0.0596 & 1.40 & 0.1561 & 1.20 & 2.6202 \\
\hline OM09-84C-MS & 0.0161 & 1.40 & 0.0041 & 1.20 & 0.2532 \\
\hline OM09-106C-MS-A (Top) & 0.8275 & 1.40 & 0.2917 & 1.20 & 0.3525 \\
\hline \multirow[t]{3}{*}{ OM09-106C-MS-B (Bottom) } & 0.3434 & 1.40 & 0.1507 & 1.20 & 0.4389 \\
\hline & 0.3456 & 1.40 & 0.1507 & 1.20 & 0.4361 \\
\hline & 0.3445 & 1.40 & 0.1507 & 1.20 & 0.4375 \\
\hline OM09-107C-MS & 0.6016 & 1.40 & 0.4250 & 1.20 & 0.7065 \\
\hline OM09-109C-MS & 0.0687 & 1.40 & 0.1072 & 1.20 & 1.5607 \\
\hline \multirow[t]{3}{*}{ OM09-10COPS-MS } & 0.2693 & 1.40 & 0.3455 & 1.20 & 1.2829 \\
\hline & 0.1732 & 1.40 & 0.3560 & 1.20 & 2.0551 \\
\hline & 0.2213 & 1.40 & 0.3507 & 1.20 & $\mathbf{1 . 5 8 5 2}$ \\
\hline \multicolumn{6}{|l|}{ Mg-Rich Carbonate Outcrop Veins: } \\
\hline \multirow[t]{3}{*}{ OM09-47C-MS } & 0.0030 & 1.40 & 0.0007 & 1.20 & 0.2317 \\
\hline & 0.0007 & 1.40 & 0.0003 & 1.20 & 0.3547 \\
\hline & 0.0019 & 1.40 & 0.0005 & 1.20 & 0.2554 \\
\hline OM10-13C-MG & 0.0010 & 1.40 & 0.0002 & 1.20 & 0.1683 \\
\hline OM10-53C-MG & 0.0032 & 1.40 & 0.0006 & 1.20 & 0.1819 \\
\hline OM09-91C-MS & 0.0114 & 1.40 & 0.0030 & 1.21 & 0.2626 \\
\hline OM10-82C-MG & 0.0009 & 1.40 & 0.0003 & 1.20 & 0.2903 \\
\hline OM10-84C-MG & 0.0010 & 1.40 & 0.0021 & 1.20 & 1.9955 \\
\hline \multicolumn{6}{|l|}{ Mg-Rich Carbonate Roadcut Veins: } \\
\hline OM09-35C-MS & 0.0001 & 1.40 & 0.0003 & 1.20 & 3.0990 \\
\hline OM09-36C-MS & 0.0001 & 1.40 & 0.0003 & 1.20 & 2.9332 \\
\hline OM09-55C-MS & 0.0008 & 1.40 & 0.0004 & 1.20 & 0.4777 \\
\hline OM09-57C-MS & 0.0053 & 1.40 & 0.0005 & 1.20 & 0.0978 \\
\hline OM09-58-MS & 0.0148 & 1.40 & 0.0002 & 1.20 & 0.0111 \\
\hline \multirow[t]{3}{*}{ OM09-63C-MS } & 0.00003 & 1.40 & 0.00006 & 1.20 & 2.1948 \\
\hline & 0.00004 & 1.40 & 0.00008 & 1.20 & 1.8916 \\
\hline & 0.00003 & 1.40 & 0.00007 & 1.20 & 2.0121 \\
\hline OM10-52C-MG & 0.0003 & 1.40 & 0.0017 & 1.20 & 5.6094 \\
\hline \multicolumn{6}{|l|}{ Peridotites: } \\
\hline OM10-1P-MG & 0.0044 & 1.40 & 0.0030 & 1.20 & 0.6741 \\
\hline OM10-2P-MG & 0.0430 & 3.04 & 0.0313 & 1.20 & 0.7278 \\
\hline OM10-13P-MG & 0.0340 & 1.40 & 0.0070 & 1.20 & 0.2055 \\
\hline OM10-17P-MG & 0.0474 & 1.40 & 0.0176 & 1.20 & 0.3724 \\
\hline \multirow[t]{2}{*}{ OM10-19P-MG } & 0.0381 & 1.40 & 0.0927 & 1.20 & 2.4353 \\
\hline & 0.0424 & 1.40 & 0.0999 & 1.20 & 2.3559 \\
\hline
\end{tabular}




\begin{tabular}{|c|c|c|c|c|c|}
\hline Sample Name: & $\mathrm{U}(\mathrm{ppm}):$ & 2 s.d. (\%): & Th (ppm): & 2 s.d. (\%): & Th/U: \\
\hline & 0.0402 & 1.40 & 0.0963 & 1.20 & 2.3934 \\
\hline OM10-22P-MG & 0.0253 & 1.40 & 0.0143 & 1.20 & 0.5644 \\
\hline OM94-74h & 0.0002 & 1.40 & 0.0095 & 1.20 & 53.0635 \\
\hline OM94-74d & 0.00002 & 1.40 & 0.0059 & 1.20 & 251.9251 \\
\hline OM94-104 & 0.0001 & 1.40 & 0.0009 & 1.21 & 16.0391 \\
\hline \multicolumn{6}{|l|}{ Serpentines: } \\
\hline OM10-27C-MG-SERP & 0.00031 & 1.40 & 0.0003 & 1.20 & 1.0655 \\
\hline OM10-54C-MG-SERP & 0.00045 & 1.40 & 0.0009 & 1.20 & 2.0371 \\
\hline \multicolumn{6}{|l|}{ Sediments: } \\
\hline OM09-8S-MS & 0.1210 & 1.40 & 0.0686 & 1.20 & 0.5669 \\
\hline OM09-12S-MS & 0.9055 & 1.40 & 1.3124 & 1.20 & 1.4494 \\
\hline \multicolumn{6}{|l|}{ Silicate Standards: } \\
\hline \multirow[t]{7}{*}{ ATHO } & 2.3698 & 1.40 & 7.154 & 1.21 & 3.0188 \\
\hline & 2.2930 & 1.40 & 6.8046 & 1.20 & 2.9676 \\
\hline & 2.228 & 1.40 & 7.2318 & 1.20 & 3.2460 \\
\hline & 2.2707 & 1.40 & 7.2409 & 1.20 & 3.1889 \\
\hline & 2.2808 & 1.40 & 7.3287 & 1.20 & 3.2132 \\
\hline & 2.2918 & 1.40 & 7.3516 & 1.20 & 3.2078 \\
\hline & 2.2890 & 1.40 & 7.1853 & 1.20 & 3.1391 \\
\hline \multirow[t]{3}{*}{ BHVO-1 } & 0.4309 & 1.40 & 1.2012 & 1.20 & 2.7878 \\
\hline & 0.4377 & 1.40 & 1.1946 & 1.20 & 2.7293 \\
\hline & 0.4343 & 1.40 & 1.1979 & 1.20 & 2.7583 \\
\hline PCC-1 & 0.0053 & 1.40 & 0.0183 & 1.20 & 3.4404 \\
\hline DTS-1 & 0.0049 & 1.40 & 0.0237 & 1.20 & 4.8193 \\
\hline \multicolumn{6}{|l|}{ Carbonate Standard: } \\
\hline \multirow[t]{5}{*}{$\mathrm{JCp}-1$} & 2.7300 & 1.40 & 0.0502 & 1.20 & 0.0184 \\
\hline & 2.7503 & 1.40 & 0.0505 & 1.20 & 0.0184 \\
\hline & 2.7616 & 1.40 & 0.0510 & 1.20 & 0.0185 \\
\hline & 2.8075 & 1.40 & 0.0572 & 1.20 & 0.0204 \\
\hline & 2.7623 & 1.40 & 0.0522 & 1.200 & 0.0189 \\
\hline
\end{tabular}

Replicate analyses are indicated in italics, with the average of replicate analyses given in bold. Numbers in red are below detection limits. 
Table 3: $\mathrm{U}$ and Th Isotopes

\begin{tabular}{|c|c|c|c|c|c|c|c|c|c|}
\hline Sample Name: & $\begin{array}{c}{ }^{234} \mathrm{U} /{ }^{238} \mathrm{U} \\
(\mathrm{ppm}):\end{array}$ & 2 s.d. (\%): & $\begin{array}{c}{ }^{230} \mathrm{Th} /{ }^{232} \mathrm{Th} \\
(\mathrm{ppm}):\end{array}$ & 2 s.d. (\%): & $\left({ }^{234} U /^{238} U\right):$ & $\left({ }^{230} \mathrm{Th} /{ }^{232} \mathrm{Th}\right):$ & $\left({ }^{238} \mathrm{U} /{ }^{232} \mathrm{Th}\right):$ & $\left({ }^{230} \mathrm{Th} /{ }^{238} \mathrm{U}\right):$ & $\begin{array}{c}\text { Uncorrected } \\
{ }^{230} \mathrm{Th} \text { Age } \\
\text { (yr): }\end{array}$ \\
\hline \multicolumn{10}{|l|}{ Travertines: } \\
\hline $\begin{array}{c}\text { OM09-76C-MS- } \\
\text { A }\end{array}$ & 64.815 & 0.069 & 7.503 & 0.226 & 1.181 & 1.389 & 2.013 & 0.690 & 93,000 \\
\hline $\begin{array}{c}\text { OM09-76C-MS- } \\
\text { C } \\
\end{array}$ & 64.296 & 0.103 & 6.344 & 0.335 & 1.172 & 1.174 & 1.252 & 0.938 & 163,000 \\
\hline OM09-84C-MS & 45.670 & 0.117 & 32.149 & 0.462 & 0.832 & 5.950 & 11.980 & 0.497 & 104,000 \\
\hline $\begin{array}{c}\text { OM09-8COPS- } \\
\text { MS }\end{array}$ & 62.190 & 0.079 & 7.434 & 0.136 & 1.133 & 1.376 & 1.158 & 1.188 & -- \\
\hline $\begin{array}{c}\text { OM09-106C- } \\
\text { MS-A } \\
\end{array}$ & 63.466 & 0.024 & 20.330 & 0.098 & 1.156 & 3.763 & 8.606 & 0.437 & 51,000 \\
\hline \multirow[t]{3}{*}{$\begin{array}{c}\text { OM09-106C- } \\
\text { MS-B }\end{array}$} & 63.427 & 0.038 & 14.707 & 0.171 & 1.156 & 2.722 & 6.912 & 0.394 & \\
\hline & 63.390 & 0.039 & 14.693 & 0.090 & 1.155 & 2.719 & 6.956 & 0.391 & \\
\hline & 63.409 & 0.039 & 14.700 & 0.131 & 1.155 & 2.721 & 6.934 & 0.392 & 45,000 \\
\hline OM09-107C-MS & 68.860 & 0.032 & 23.603 & 0.082 & 1.255 & 4.369 & 4.294 & 1.017 & 164,000 \\
\hline OM09-109C-MS & 61.355 & 0.059 & 7.694 & 0.218 & 1.118 & 1.424 & 1.944 & 0.733 & 113,000 \\
\hline $\begin{array}{c}\text { OM09-10COPS- } \\
\text { MS }\end{array}$ & 61.451 & 0.041 & 7.649 & 0.153 & 1.120 & 1.416 & 1.476 & 0.959 & -- \\
\hline \multicolumn{10}{|l|}{$\begin{array}{c}\text { Mg-Rich Outcrop } \\
\text { Veins: }\end{array}$} \\
\hline OM09-47C-MS & 67.905 & 0.148 & 8.666 & 0.456 & 1.237 & 1.604 & 8.553 & 0.188 & 18,000 \\
\hline OM10-13C-MG & 67.111 & 0.093 & 31.111 & 0.800 & 1.223 & 5.758 & 18.024 & 0.319 & 33,000 \\
\hline OM10-53C-MG & 66.747 & 0.099 & 8.312 & 0.629 & 1.216 & 1.538 & 16.680 & 0.092 & 9,000 \\
\hline OM09-91C-MS & 66.866 & 0.065 & 12.481 & 0.356 & 1.218 & 2.310 & 11.551 & 0.200 & 19,000 \\
\hline OM10-82C-MG & 67.717 & 0.131 & -- & -- & 1.234 & -- & 10.450 & -- & -- \\
\hline OM10-84C-MG & 65.139 & 0.134 & 6.723 & 0.374 & 1.187 & 1.244 & 1.520 & 0.818 & 122,000 \\
\hline
\end{tabular}




\begin{tabular}{|c|c|c|c|c|c|c|c|c|c|}
\hline Sample Name: & $\begin{array}{l}{ }^{234} U /{ }^{238} U \\
\text { (ppm): }\end{array}$ & 2 s.d. (\%): & $\begin{array}{c}{ }^{230} \mathrm{Th} /{ }^{232} \mathrm{Th} \\
\text { (ppm): }\end{array}$ & 2 s.d. (\%): & $\left({ }^{234} U /{ }^{238} U\right):$ & $\left({ }^{230} \mathrm{Th} /{ }^{232} \mathrm{Th}\right):$ & $\left({ }^{238} \mathrm{U} /{ }^{232} \mathrm{Th}\right):$ & $\left({ }^{230} \mathrm{Th} /{ }^{238} \mathrm{U}\right):$ & $\begin{array}{c}\text { Uncorrected } \\
{ }^{230} \mathrm{Th} \text { Age } \\
\text { (yr): }\end{array}$ \\
\hline \multicolumn{10}{|l|}{$\begin{array}{l}\text { Mg-Rich Roadcut } \\
\text { Veins: }\end{array}$} \\
\hline OM09-35C-MS & -- & -- & 5.650 & 1.435 & -- & 1.046 & 0.979 & 1.068 & -- \\
\hline OM09-55C-MS & 6.630 & 1.896 & 32.800 & 0.785 & 1.044 & 6.071 & 6.351 & 0.956 & 256,000 \\
\hline OM09-57C-MS & 31.344 & 1.847 & 164.870 & 0.151 & 1.010 & 30.515 & 31.019 & 0.984 & $>375,000$ \\
\hline OM09-58C-MS & 54.751 & 0.043 & -- & -- & 0.998 & -- & 272.445 & -- & -- \\
\hline OM10-52C-MG & 61.609 & 0.189 & 4.506 & 0.428 & 1.123 & 0.834 & 0.541 & 1.542 & $\begin{array}{c}\text { Not } \\
\text { Determinable }\end{array}$ \\
\hline \multicolumn{10}{|l|}{ Peridotites: } \\
\hline OM10-1P-MG & 51.173 & 0.334 & -- & -- & 0.932 & -- & 4.501 & -- & -- \\
\hline OM10-2P-MG & 68.671 & 0.125 & 7.503 & 0.249 & 1.251 & 1.389 & 4.169 & 0.333 & -- \\
\hline OM10-13P-MG & 62.389 & 0.067 & 66.817 & 0.168 & 1.137 & 12.367 & 14.763 & 0.838 & -- \\
\hline OM10-17P-MG & 65.831 & 0.069 & 8.175 & 0.400 & 1.199 & 1.513 & 8.146 & 0.186 & -- \\
\hline \multirow[t]{3}{*}{ OM10-19P-MG } & 62.663 & 0.125 & - & -- & 1.142 & -- & 1.246 & -- & \\
\hline & 58.718 & 0.135 & -- & -- & 1.070 & -- & 1.288 & -- & \\
\hline & 60.691 & 0.130 & -- & - & 1.106 & -- & 1.267 & -- & -- \\
\hline OM10-22P-MG & 66.522 & 0.076 & 7.647 & 0.280 & 1.212 & 1.415 & 5.375 & 0.263 & -- \\
\hline \multicolumn{10}{|l|}{ Sediments: } \\
\hline OM09-8S-MS & 56.637 & 0.033 & 28.178 & 0.075 & 1.032 & 5.215 & 5.352 & 0.975 & -- \\
\hline OM09-12S-MS & 60.501 & 0.028 & 8.621 & 0.063 & 1.102 & 1.596 & 2.093 & 0.762 & -- \\
\hline \multicolumn{10}{|l|}{$\begin{array}{c}\text { Silicate } \\
\text { Standards: }\end{array}$} \\
\hline \multirow[t]{4}{*}{ ATHO } & 54.992 & 0.031 & 5.527 & 0.077 & 1.002 & 1.023 & 0.935 & 1.094 & \\
\hline & 55.061 & 0.040 & 5.563 & 0.063 & 1.003 & 1.030 & 0.951 & 1.082 & \\
\hline & 54.934 & 0.059 & 5.439 & 0.059 & 1.001 & 1.007 & 0.946 & 1.064 & \\
\hline & 54.996 & 0.043 & 5.509 & 0.066 & 1.002 & 1.020 & 0.944 & 1.080 & -- \\
\hline BHVO-1 & 54.988 & 0.0552 & 5.843 & 0.158 & 1.002 & 1.081 & 1.088 & 0.994 & \\
\hline
\end{tabular}




\begin{tabular}{|c|c|c|c|c|c|c|c|c|c|}
\hline Sample Name: & $\begin{array}{l}{ }^{234} \mathrm{U} / /^{238} \mathrm{U} \\
\text { (ppm): }\end{array}$ & 2 s.d. (\%): & $\begin{array}{c}{ }^{230} \mathrm{Th} /{ }^{232} \mathrm{Th} \\
\text { (ppm): }\end{array}$ & 2 s.d. (\%): & $\left({ }^{234} U{ }^{238} U\right):$ & $\left({ }^{230} \mathrm{Th} /{ }^{232} \mathrm{Th}\right):$ & $\left({ }^{238} U /^{232} T h\right):$ & $\left({ }^{230} \mathrm{Th} /{ }^{238} \mathrm{U}\right)$ & $\begin{array}{c}\text { Uncorrected } \\
{ }^{230} \mathrm{Th} \text { Age } \\
(\mathrm{yr}):\end{array}$ \\
\hline & 54.908 & 0.038 & 5.848 & 0.158 & 1.000 & 1.082 & 1.112 & 0.974 & \\
\hline & 54.948 & 0.046 & 5.846 & 0.158 & 1.001 & 1.082 & 1.100 & 0.984 & -- \\
\hline PCC-1 & 57.344 & 0.348 & --- & -- & 1.045 & -- & 0.882 & -- & -- \\
\hline DTS-1 & 54.802 & 0.377 & -- & -- & 0.999 & -- & 0.629 & -- & -- \\
\hline & & & & & & & & & \\
\hline \multicolumn{10}{|l|}{$\begin{array}{l}\text { Carbonate } \\
\text { Standard: }\end{array}$} \\
\hline \multirow[t]{5}{*}{ JCp-1 } & 63.002 & 0.048 & 6.965 & 0.849 & 1.148 & 1.289 & 164.831 & 0.008 & \\
\hline & 62.943 & 0.040 & 7.016 & 0.430 & 1.148 & 1.299 & 165.235 & 0.008 & \\
\hline & 63.017 & 0.029 & 6.898 & 0.368 & 1.148 & 1.277 & 164.308 & 0.008 & \\
\hline & 62.899 & 0.024 & 7.107 & 0.420 & 1.146 & 1.315 & 148.854 & 0.009 & \\
\hline & 62.965 & 0.035 & 6.997 & 0.517 & 1.148 & 1.295 & 160.807 & 0.008 & 760 \\
\hline & & & & & & & & & \\
\hline
\end{tabular}

Replicate analyses are indicated in italics, with the average of replicate analyses given in bold. Parentheses indicate activities. Due to the uncertainties in determining ${ }^{230} \mathrm{Th}$ ages for Samail carbonates, the uncorrected ${ }^{230} \mathrm{Th}$ ages are presented to the nearest 1,000 years and with no associated errors. 


\section{Figures:}

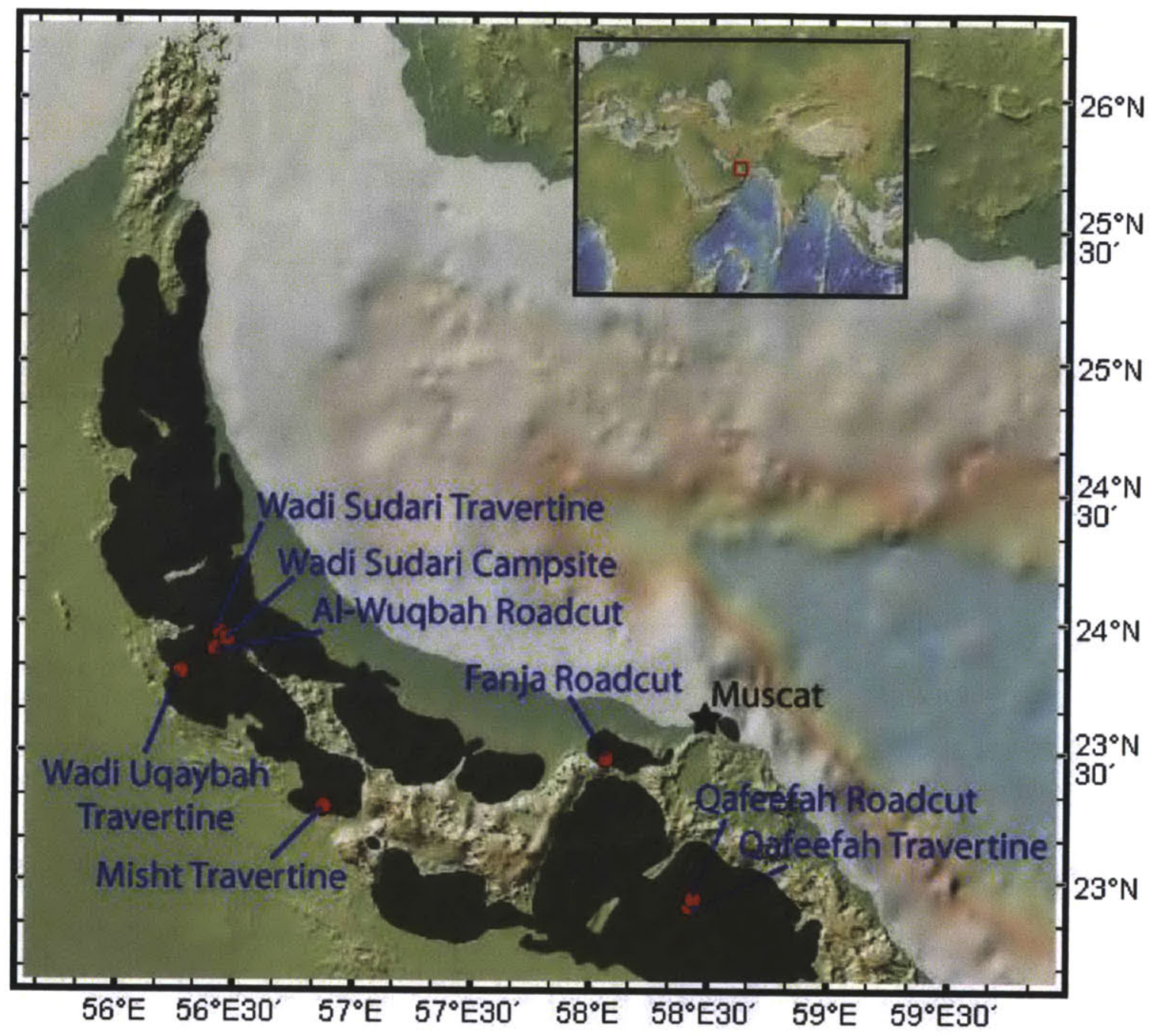

Figure 1: The eight field locations where carbonate and peridotite samples were collected during 2009 and 2010 field seasons. The Samail Ophiolite is outlined in dark gray, and sampling locations are indicated by red circles. Base map from GeoMapApp. 


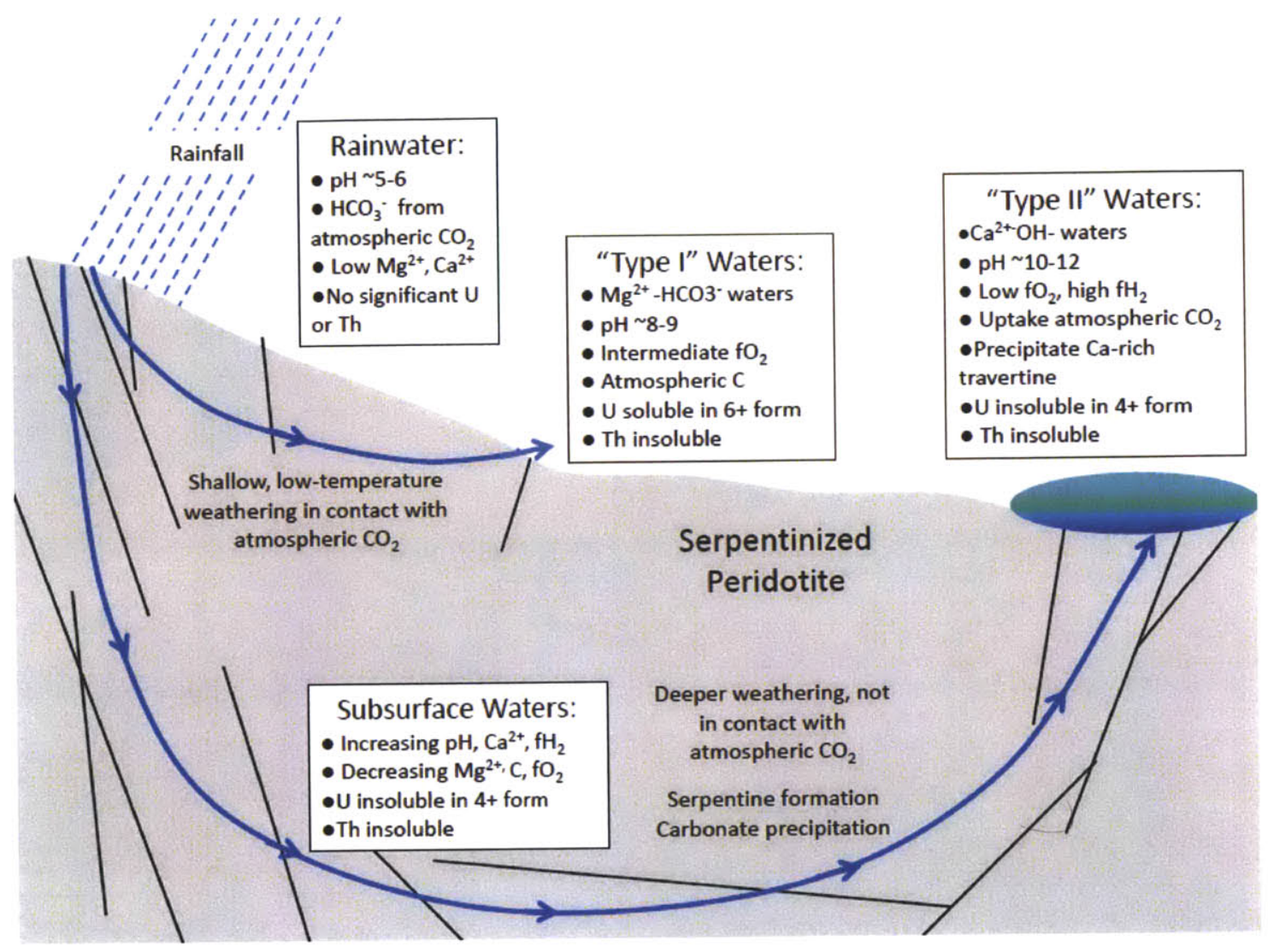

Figure 2: Schematic illustrating the behaviour of U and Th in Type I and Type II waters associated with the alteration of partially-serpentinized peridotite. Figure modified from Neal and Stanger (1985). 


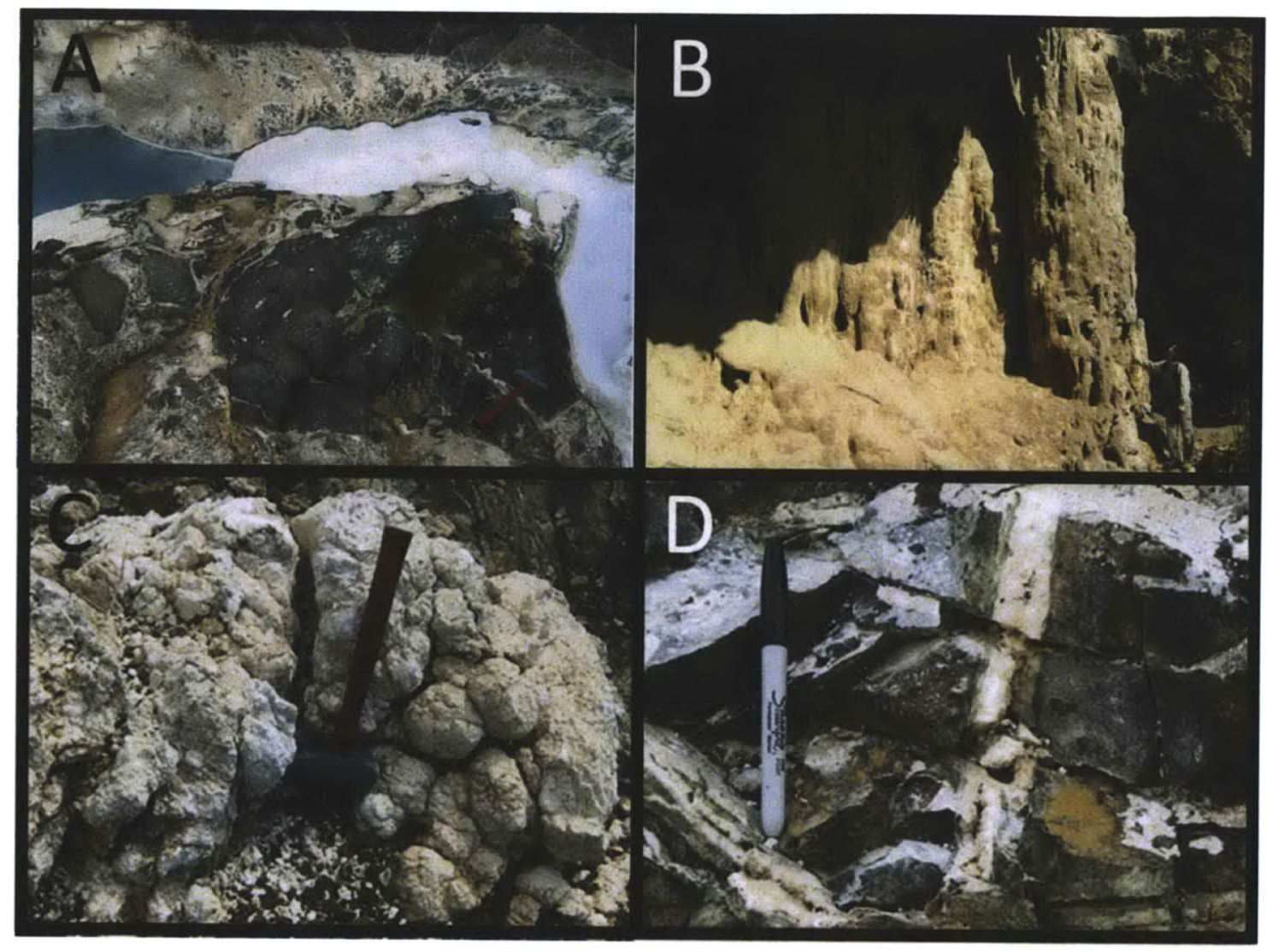

Figure 3: Representative images of carbonate alteration products formed in the peridotie layer of the Samail Ophiolite. A: A turquoise alkaline pool and recently-formed travertine precipitate at Wadi Sudari Travertine. Note the hammer for scale. B: Travertine pseudospeleothems forming underneath a travertine terrace at Wadi Sudari Travertine. Note the person for scale. C: A white magnesite vein with botryoidal texture exposed on the natural peridotite weathering surface near Fanja Roadcut. Note the hammer for scale. D: Dolomite-serpentine veins exposed on the surface of Fanja Roadcut. Note the Sharpie marker for scale. 


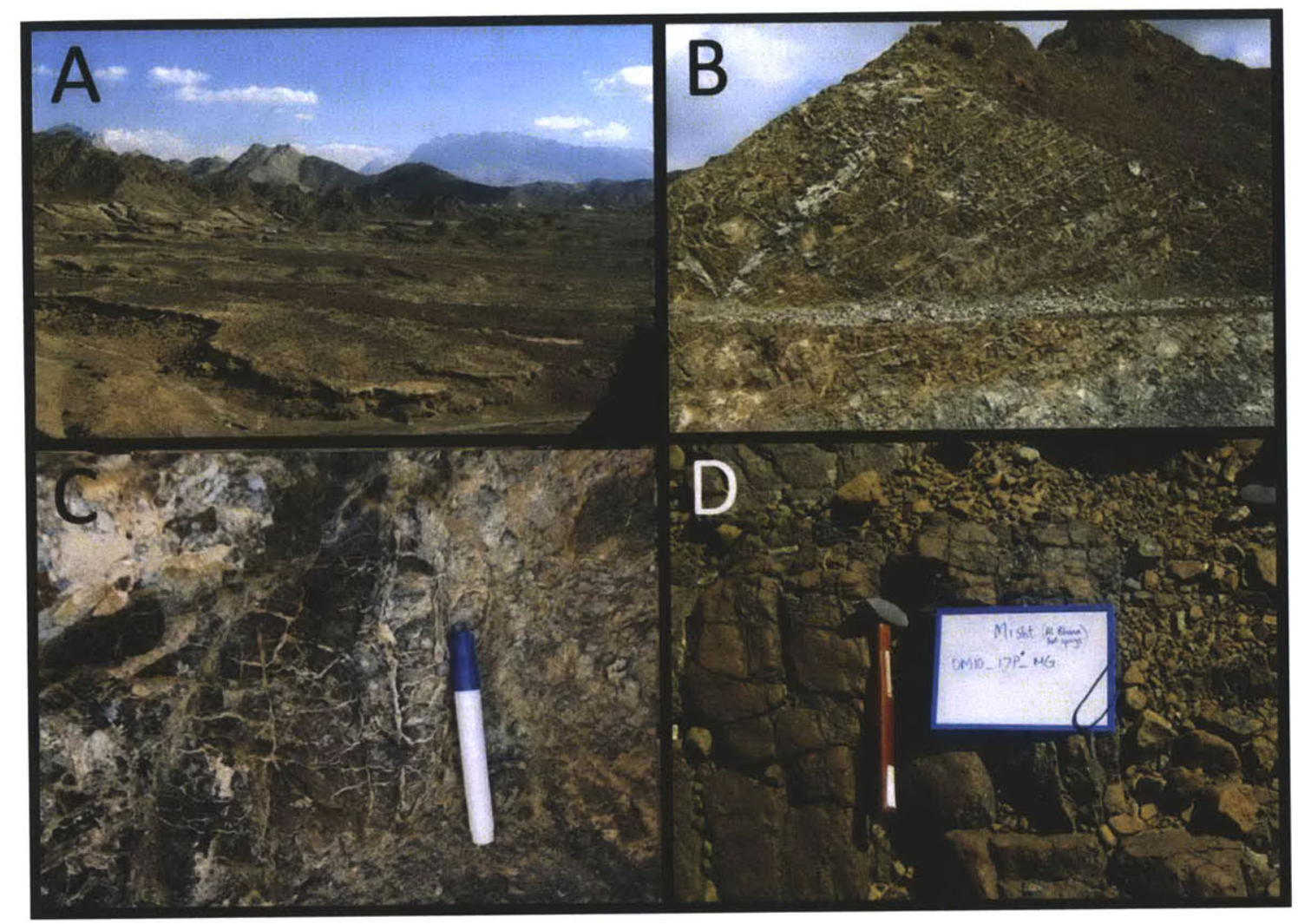




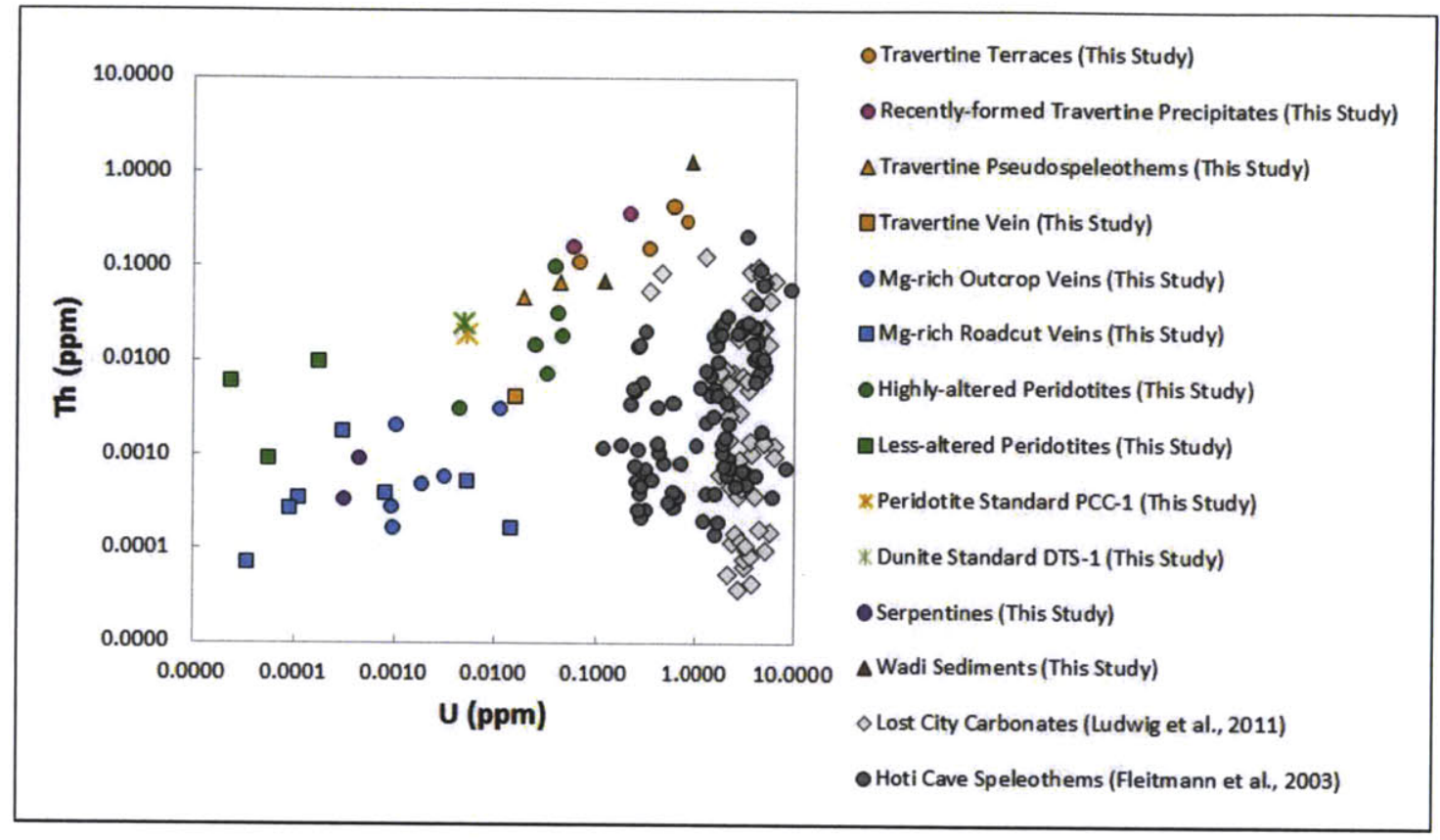

Figure 5: Plot of $U$ and Th concentrations for Samail travertines (orange circles for travertine terraces, pink circles for recently-formed travertine precipitates, orange triangles for travertine pseudoespeleothems, and an orange square for the travertine vein), carbonate veins (blue circles for outcrop veins and blue squares for roadcut veins), serpentine mineral separates from carbonate-serpentine veins (purple circles), highlyaltered peridotites (green circles), less-altered peridotites (green squares), peridotite standard PCC-1 (orange star), dunite standard DTS-1 (green star), and wadi sediments (brown triangles). Internal 2 s.d. error bars are smaller than the symbol sizes. Note that both axes have a $\log$ scale. Shown for reference are $U$ and $T h$ concentrations for carbonates formed at Lost City, a hydrothermal site located in peridotite $\sim 15 \mathrm{~km}$ off-axis along the Mid-Atlantic Ridge (Ludwig et al., 2011) and in speleothems from Hoti Cave, which formed in Cretaceous age Natih limestone in northern Oman (Fleitmann et al., 2003). 


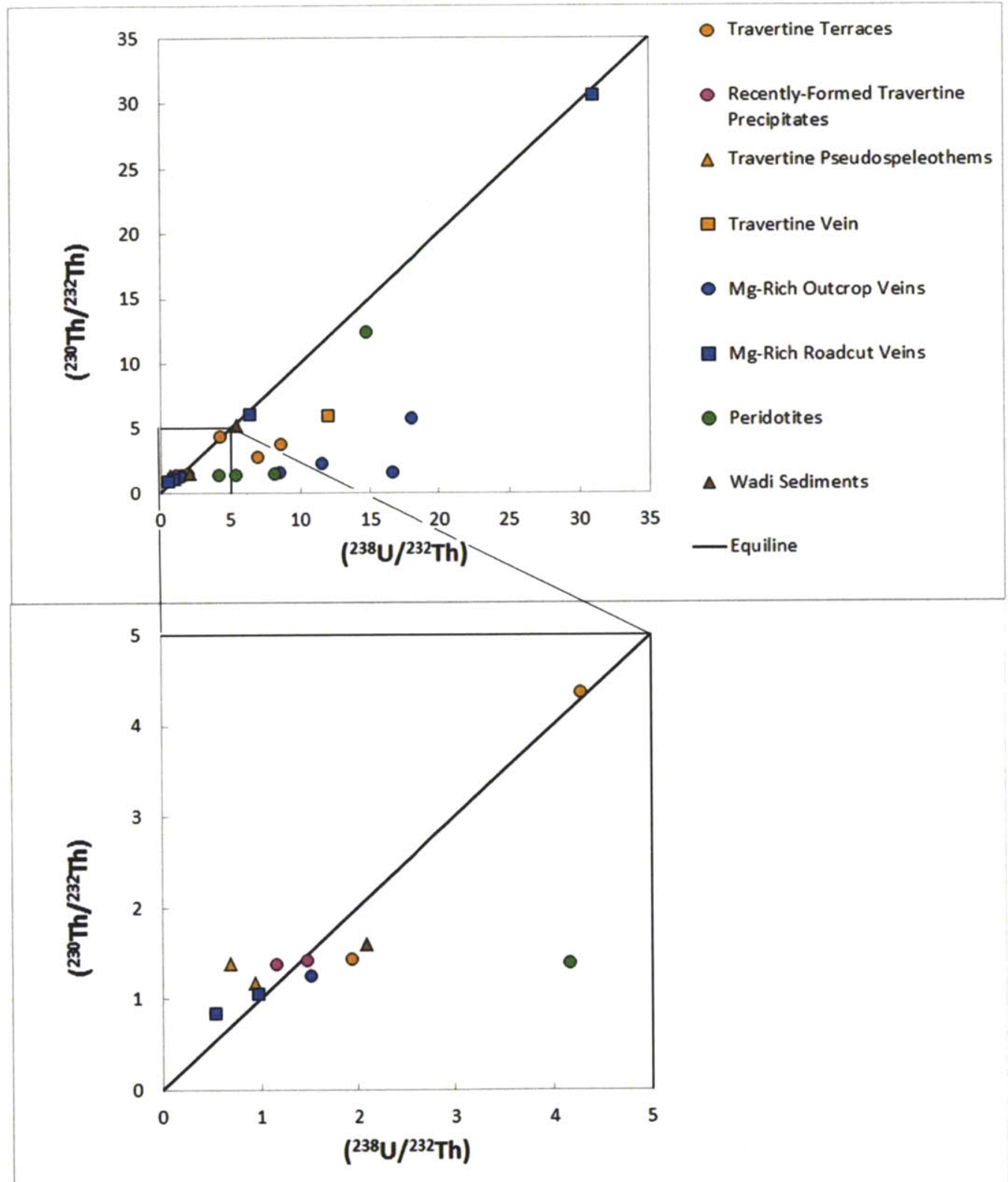




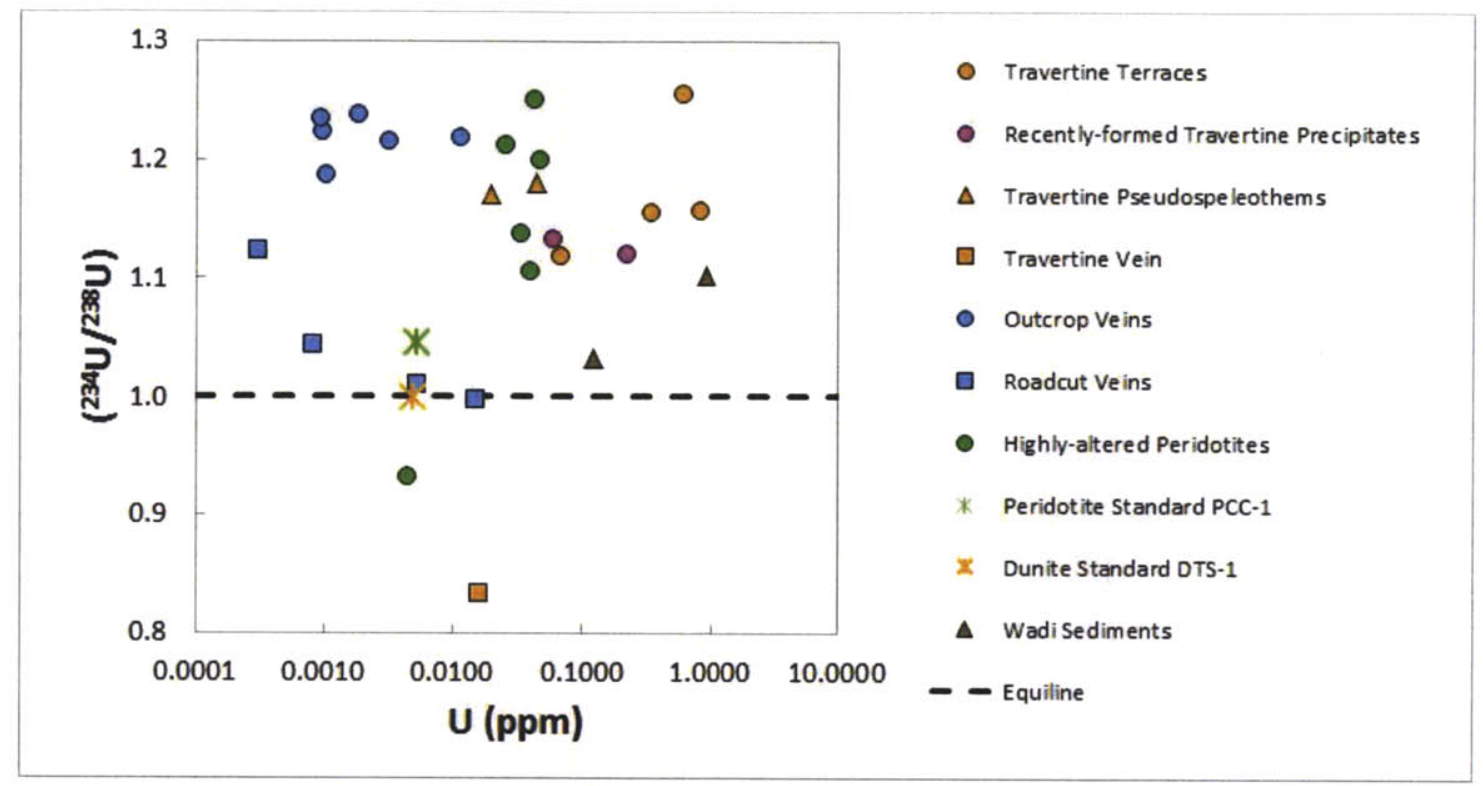

Figure 7: Plot of $\left({ }^{234} U{ }^{238} U\right)$ versus $U$ concentration for Samail travertines, carbonate veins, highly-altered peridotites, and wadi sediments using the same symbol scheme as Figure 5. Internal 2 s.d. error bars are smaller than the symbol sizes. The dashed line indicates equilibrium for $\left({ }^{234} \mathrm{U} /{ }^{238} \mathrm{U}\right)$. 


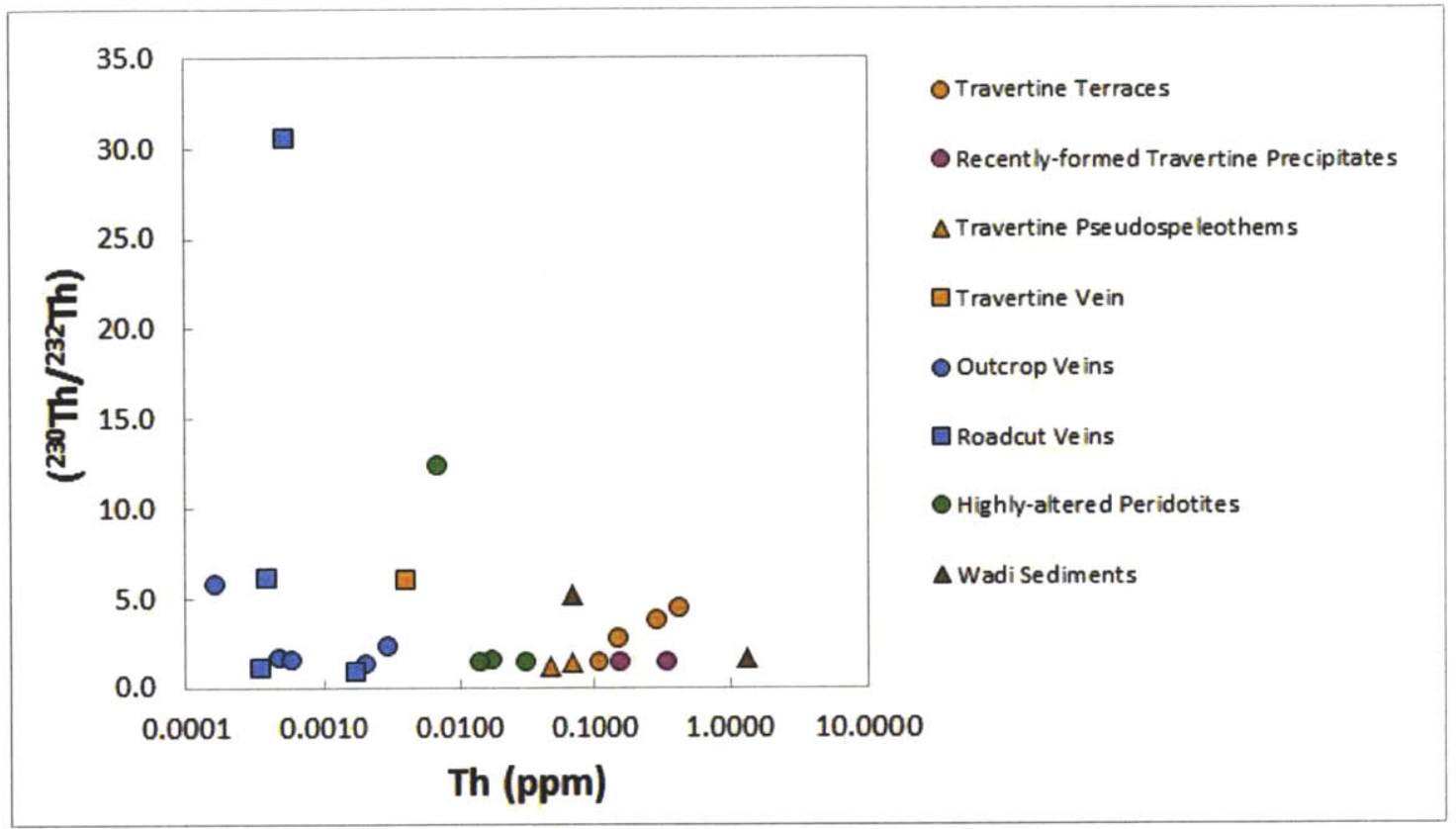

Figure 8: Plot of $\left({ }^{230} \mathrm{Th} /{ }^{232} \mathrm{Th}\right)$ versus Th concentration for Samail travertines, carbonate veins, highly-altered peridotites, and wadi sediments using the same symbol scheme as Figure 5. Internal 2 s.d. error bars are smaller than the symbol sizes. 


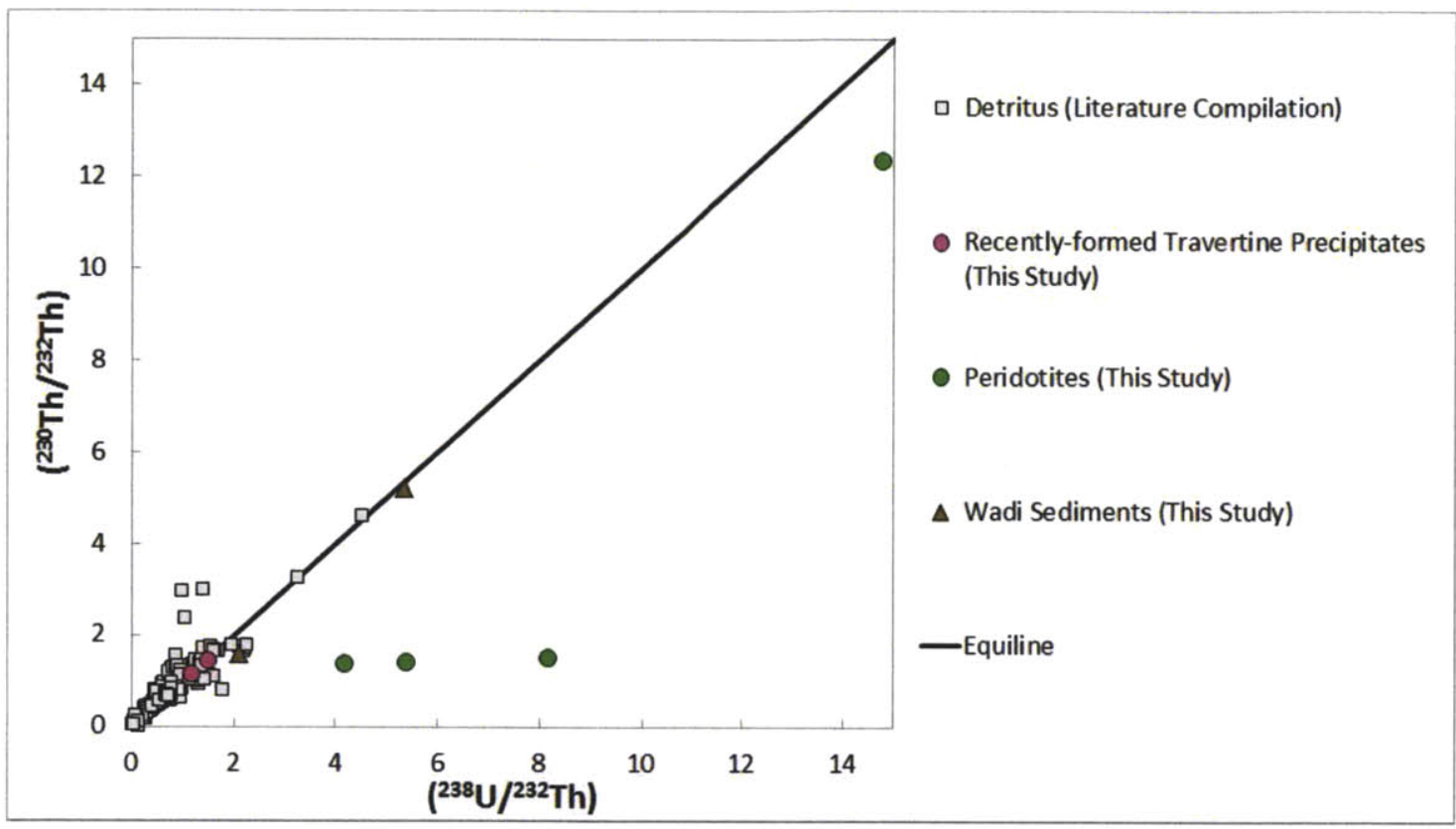

Figure 9: Plot of $\left({ }^{230} \mathrm{Th} /{ }^{232} \mathrm{Th}\right)$ versus $\left({ }^{238} \mathrm{U} /{ }^{232} \mathrm{Th}\right)$ for a compilation of 300 literature values for aluminosilicate detritus including aeolian soil (loess), glacial till, alluvium, colluvium, and other soil. The literature compilation includes both alpha counting and mass spectrometric measurements and was compiled from Rosholt et al., 1966; Hansen and Stout, 1968; Hansen, 1970; Rosholt et al., 1985; Mathieu et al., 1995; Dequincey et al., 2002; Krishnaswami et al., 2004; Dosseto et al., 2008; Pelt et al., 2008; Ma et al., 2010. For comparison, recently-formed travertine precipitates, highly-altered peridoties, wadi sediments from the peridotite layer of the Samail Ophiolite are also plotted. 


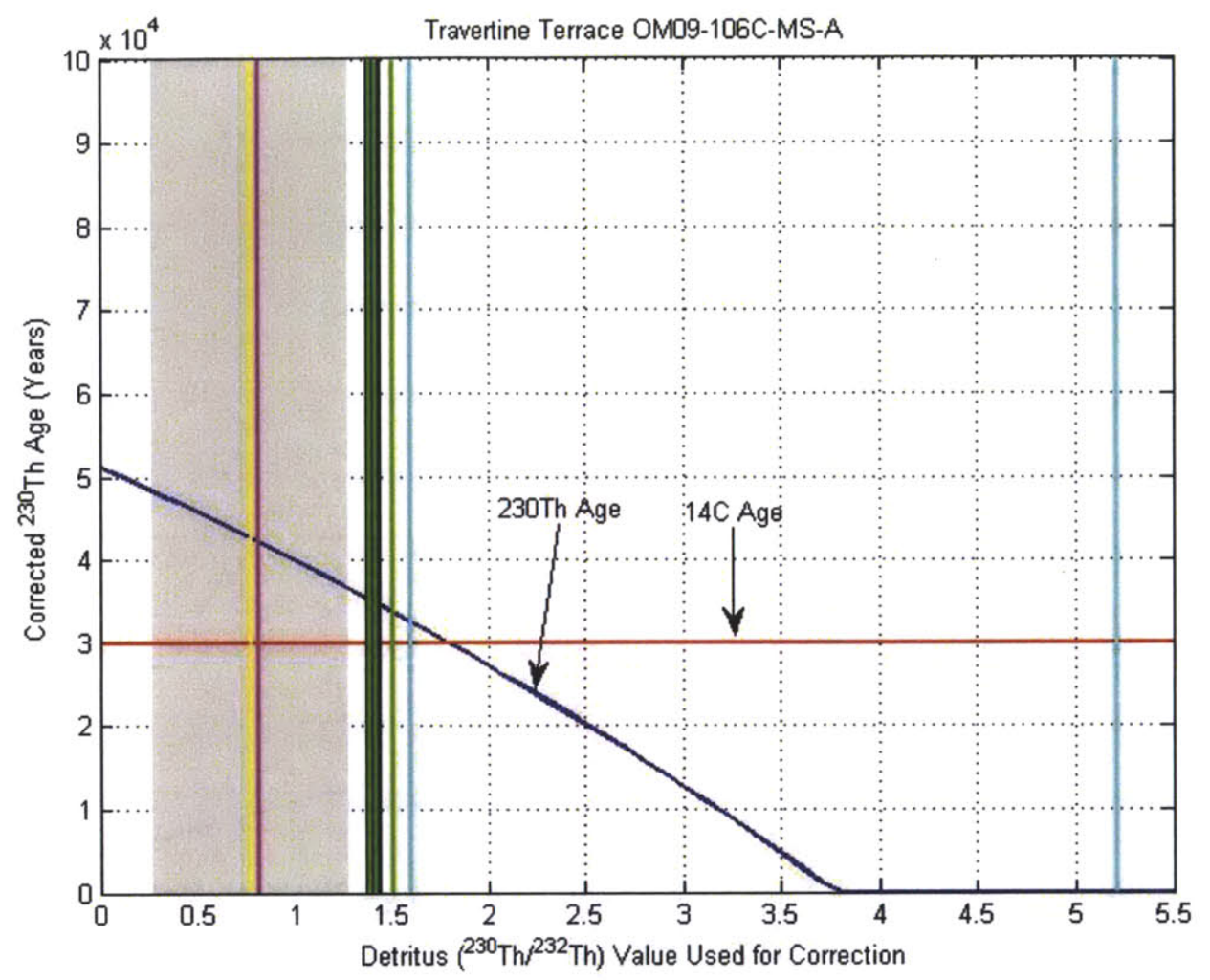

Figure 10: Sensitivity analysis figure showing the effect of the selected $\left({ }^{230} \mathrm{Th} /{ }^{232} \mathrm{Th}\right)$ correction value on the corrected ${ }^{230} \mathrm{Th}$ age calculated using Equation 1. This figure is for travertine Sample OM09-106C-MS-A, a travertine terrace from Wadi Uqaybah. The dark blue line indicates the corrected ${ }^{230} \mathrm{Th}$ age. Note that the uncorrected ${ }^{230} \mathrm{Th}$ age plots at $\mathrm{x}$ $=0$. For comparison, the ${ }^{14} \mathrm{C}$ is plotted as the red line. The gray box indicates the $2 \sigma$ range of $\left({ }^{230} \mathrm{Th} /{ }^{232} \mathrm{Th}\right)$ for aluminosilicate detritus (literature compilation shown in Figure $9, \mathrm{n}=300)$. Vertical lines indicate possible values to use for the $\left({ }^{230} \mathrm{Th} /{ }^{232} \mathrm{Th}\right)$ correction value: average of aluminosilicate detritus compilation (yellow line), equilibrium Bulk Silicate Earth (pink line), highly-altered peridotites (green lines; note that a fourth peridotite plots off the figure at 12.367), wadi sediments (turquoise lines), and recentlyformed travertine precipitates (black lines). The choice of the $\left({ }^{230} \mathrm{Th} /{ }^{232} \mathrm{Th}\right)$ correction value has a very significant impact on the determined ${ }^{230} \mathrm{Th}$ age, which ranges from $\sim 50,000$ years to zero depending on the correction value selected. However, a $\left({ }^{230} \mathrm{Th} /{ }^{232} \mathrm{Th}\right)$ correction value of approximately 1.7 brings the corrected ${ }^{230} \mathrm{Th}$ age into concordance with the ${ }^{14} \mathrm{C}$ age. 


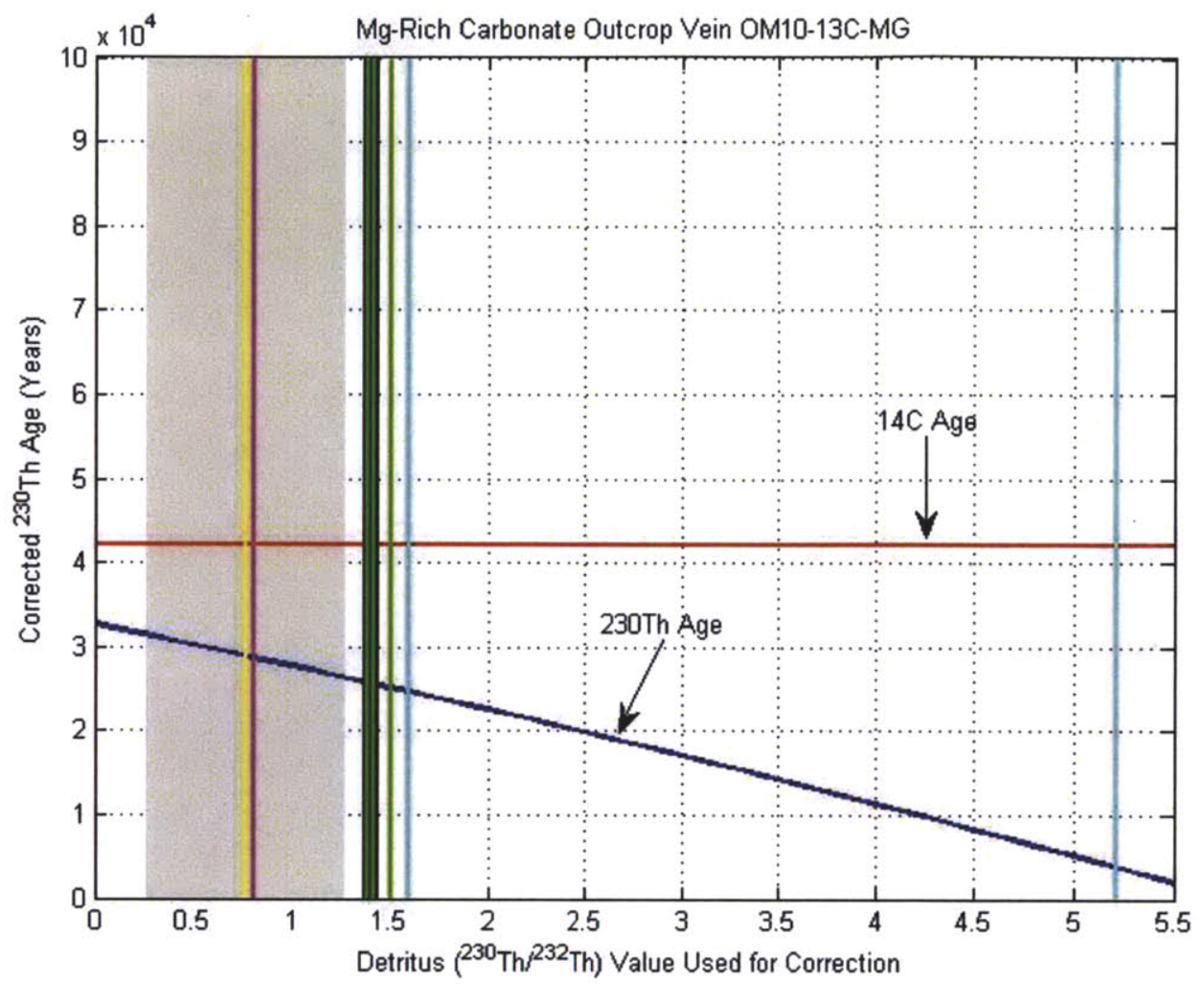

Figure 11: Sensitivity analysis figure showing the effect of the selected $\left({ }^{230} \mathrm{Th} /{ }^{232} \mathrm{Th}\right)$ correction value on the corrected ${ }^{230} \mathrm{Th}$ age calculated using Equation 1. This figure is for Sample OM10-13C-MG, an Mg-rich carbonate outcroup vein from Fanja Roadcut. The dark blue line indicates the corrected ${ }^{230} \mathrm{Th}$ age. Note that the uncorrected ${ }^{230} \mathrm{Th}$ age plots at $\mathrm{x}=0$. For comparison, the ${ }^{14} \mathrm{C}$ age is plotted as the red line. The gray box indicates the $2 \sigma$ range of $\left({ }^{230} \mathrm{Th} /{ }^{232} \mathrm{Th}\right)$ for aluminosilicate detritus (literature compilation shown in Figure 9, n = 300). Vertical lines indicate possible values to use for the $\left({ }^{230} \mathrm{Th} /{ }^{232} \mathrm{Th}\right)$ correction value: average of aluminosilicate detritus compilation (yellow line), equilibrium Bulk Silicate Earth (pink line), highly-altered peridotites (green lines; note that a fourth peridotite plots off the figure at 12.367), wadi sediments (turquoise lines), and recently-formed travertine precipitates (black lines). The choice of the $\left({ }^{230} \mathrm{Th} /{ }^{232} \mathrm{Th}\right.$ ) correction value has a very significant impact on the determined ${ }^{230} \mathrm{Th}$ age, which ranges from $\sim 30,000$ years to zero depending on the correction value selected. Since the uncorrected ${ }^{230} \mathrm{Th}$ age is younger than the ${ }^{14} \mathrm{C}$ age, no ${ }^{230} \mathrm{Th}$ correction can bring the two ages into concordance. 


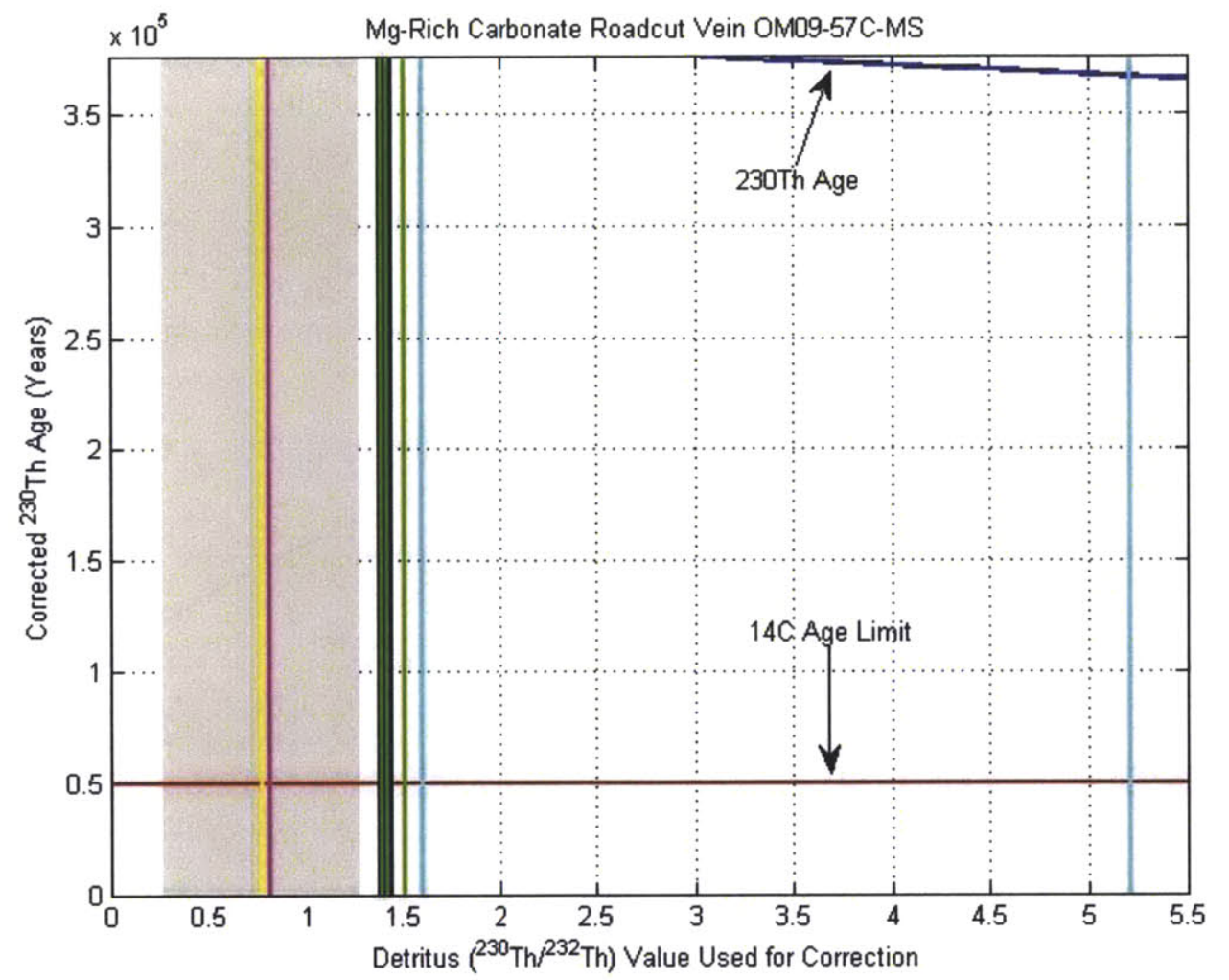

Figure 12: Sensitivity analysis figure showing the effect of the selected $\left({ }^{230} \mathrm{Th} /{ }^{232} \mathrm{Th}\right)$ correction value on the corrected ${ }^{230} \mathrm{Th}$ age calculated using Equation 1. This figure is for sample OM09-57C-MS, an Mg-rich carbonate roadcut vein from Fanja Roadcut. The dark blue line indicates the corrected ${ }^{230} \mathrm{Th}$ age. Note that the uncorrected ${ }^{230} \mathrm{Th}$ age plots at $\mathrm{x}=0$. For comparison, the ${ }^{14} \mathrm{C}$ age is plotted as the red line. The gray box indicates the $2 \sigma$ range of $\left({ }^{230} \mathrm{Th} /{ }^{232} \mathrm{Th}\right)$ for aluminosilicate detritus (literature compilation shown in Figure 9, $\mathrm{n}=300)$. Vertical lines indicate possible values to use for the $\left({ }^{230} \mathrm{Th} /{ }^{232} \mathrm{Th}\right)$ correction value: average of aluminosilicate detritus compilation (yellow line), equilibrium Bulk Silicate Earth (pink line), highly-altered peridotites (green lines; note that a fourth peridotite plots off the figure at 12.367), wadi sediments (turquoise lines), and recently-formed travertine precipitates (black lines). The choice of the $\left({ }^{230} \mathrm{Th} /{ }^{232} \mathrm{Th}\right.$ ) correction value has minimal impact on the determined ${ }^{230} \mathrm{Th}$ age, which remains greater than 350,000 years. This is consistent with the ${ }^{14} \mathrm{C}$ dead $(>50,000 \mathrm{yr} \mathrm{BP})$ age of this sample 


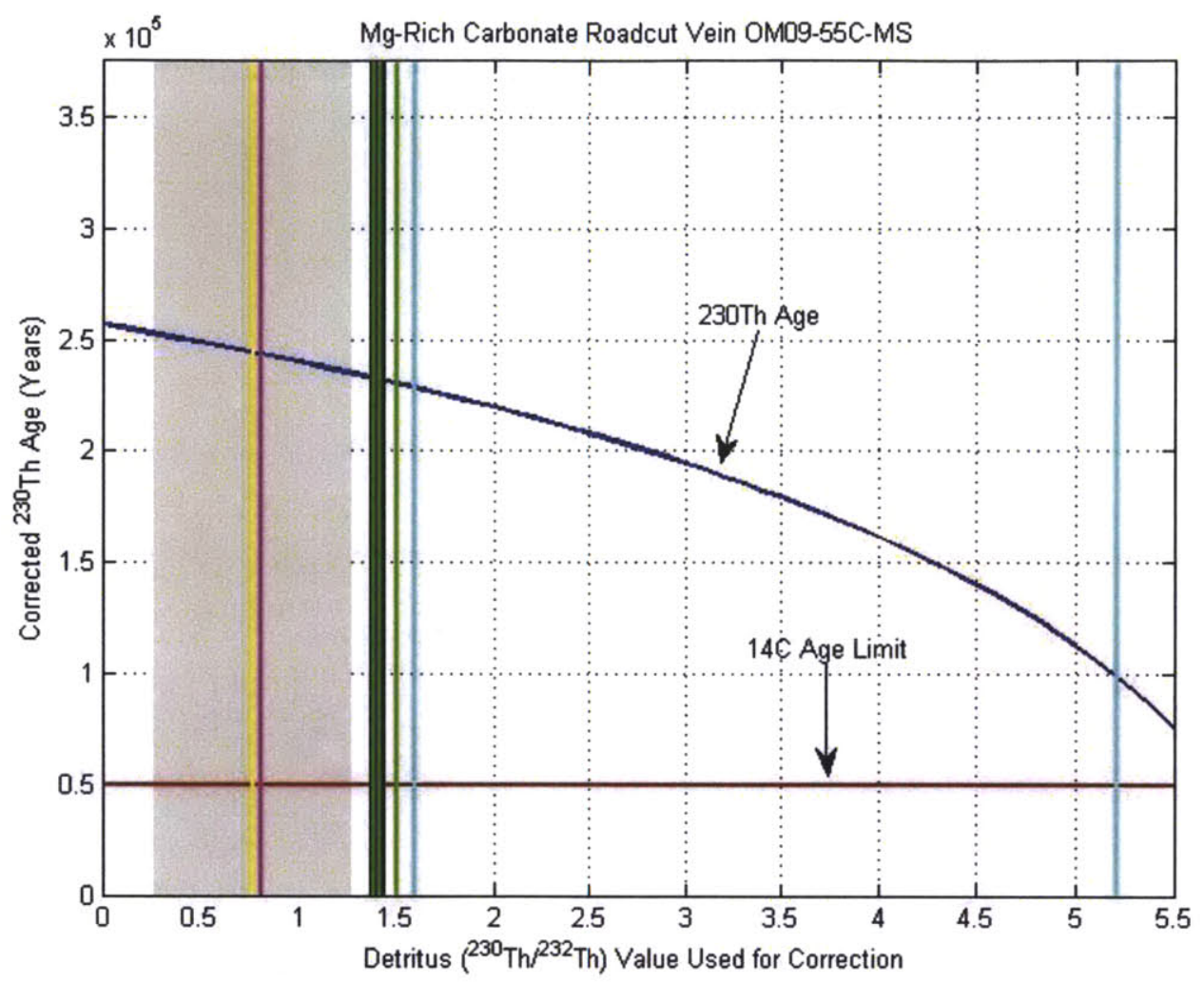

Figure 13: Sensitivity analysis figure showing the effect of the selected $\left({ }^{230} \mathrm{Th} /{ }^{232} \mathrm{Th}\right)$ correction value on the corrected ${ }^{230} \mathrm{Th}$ age calculated using Equation 1. This figure is for sample OM09-55C-MS, an Mg-rich carbonate roadcut vein from Fanja Roadcut. The dark blue line indicates the corrected ${ }^{230} \mathrm{Th}$ age. Note that the uncorrected ${ }^{230} \mathrm{Th}$ age plots at $\mathrm{x}=0$. For comparison, the ${ }^{14} \mathrm{C}$ age is plotted as the red line. The gray box indicates the $2 \sigma$ range of $\left({ }^{230} \mathrm{Th} /{ }^{232} \mathrm{Th}\right)$ for aluminosilicate detritus (literature compilation shown in Figure 9, $\mathrm{n}=300)$. Vertical lines indicate possible values to use for the $\left({ }^{230} \mathrm{Th} /{ }^{232} \mathrm{Th}\right)$ correction value: average of aluminosilicate detritus compilation (yellow line), equilibrium Bulk Silicate Earth (pink line), highly-altered peridotites (green lines; note that a fourth peridotite plots off the figure at 12.367), wadi sediments (turquoise lines), and recently-formed travertine precipitates (black lines). The choice of the $\left({ }^{230} \mathrm{Th} /{ }^{232} \mathrm{Th}\right.$ ) correction value has a significant impact on the determined ${ }^{230} \mathrm{Th}$ age, which ranges from $\sim 250,000$ years to $\sim 60,000$ years. This is consistent with the ${ }^{14} \mathrm{C}$ dead $(>50,000$ yr BP) age of this sample. 


\section{References:}

Ball, L., Sims, K.W.W., and Schwieters, J. 2008. Measurement of ${ }^{234} \mathrm{U} /{ }^{238} \mathrm{U}$ and ${ }^{230} \mathrm{Th} /{ }^{232} \mathrm{Th}$ in volcanic rocks using the Neptune MC-ICP-MS. Journal of Analytical Atomic Spectrometry, Vol. 23: 173-180.

Barnes, I., LaMarche, V.C., and Himmelberg, G. 1967. Geochemical evidence of present day serpentinization. Science, Vol. 156: 830-832.

Barnes, I. and O'Neil, J.R. 1969. The relationship between fluids in some fresh Alpine type ultramafics and possible modern serpentinization, Western United States. Geological Society of America Bulletin, Vol. 80: 1947-1960.

Barnes, I., O’Neil, J.R., and Trescases, J.J. 1978. Present-day serpentinization in New Caledonia, Oman, and Yugoslavia. Geochimica et Cosmochimia Acta, Vol. 42: 144-145.

Bischoff, J.L. and Fitzpatrick, J.A. 1991. U-series dating of impure carbonates: An isochron technique using total-sample dissolution. Geochimica et Cosmochimica Acta, Vol. 55: 543-554.

Bruni, J., Canepa, M., Chiodini, G., Cioni, R., Cipolli, F., Longinelli, A., Marini, L., Ottonello, G., and Zuccolini, M.V. 2002. Irreversible water-rock mass transfer accompanying the generation of the neutral, $\mathrm{Mg}-\mathrm{HCO}_{3}$ and high-pH, $\mathrm{Ca}-\mathrm{OH}$ spring waters of the Genova province, Italy. Applied Geochemistry, Vol. 17: 455 474.

Cheng, H., Edwards, R.L., Hoff, J., Gallup, C.D., Richards, D.A., and Asmerom, Y. 2000. The half-lives of uranium-234 and thorium-230. Chemical Geology, Vol. 169: 17-33.

Clark, I.D. and Fontes, J.-C. 1990. Paleoclimatic reconstruction in northern Oman based on carbonates from hyperalkaline groundwaters. Quaternary Research, Vol. 33: 320-336.

Clark, I.D., Fontes, J.-C., and Fritz, P. 1992. Stable isotope disequilibria in travertine from high $\mathrm{pH}$ waters: Laboratory investigations and field observations from Oman. Geochimica et Cosmochimica Acta, Vol. 56: 2041-2050.

Cipolli, F., Gambardella, B., Marini, L., Otonello, G., and Zuccolini, M.V. 2004. Geochemistry of high-pH waters from serpentinites of the Gruppo di Voltri (Genova, Italy) and reaction path modeling of $\mathrm{CO}_{2}$ sequestration in serpentine aquifers. Applied Geochemistry, Vol. 19: 787-802. 
Coleman, R.G. 1981. Tectonic setting for ophiolite obduction in Oman. Journal of Geophysical Research, Vol. 86, No. B4: 2497-2508.

Dequincey, O., Chabaux, F., Clauer, N., Sigmarsson, O., Liewig, N., and Leprun, J.-C. 2002. Chemical mobilizations in laterites: Evidence from trace elements and ${ }^{238} \mathrm{U}$ ${ }^{234} \mathrm{U}{ }^{230}$ Th disequilibria. Geochimica et Cosmochimica Acta, Vol. 66, No.7: 11971210.

Dooley, J.R., Tatsumoto, M., and Rosholt, J.N. 1964. Radioactive disequilibrium studies of roll features, Shirley Basin, Wyoming. Economic Geology, Vol. 59: 586-595.

Dooley, J.R., Granger, H.C., and Rosholt, J.N. 1966. Uranium-234 fractionation in the sandstone-type uranium deposits of the Ambrosia Lake District, New Mexico. Economic Geology, Vol. 61: 1362-1382.

Dossetto, A., Turner, S.P., and Chappell, J. 2008. The evolution of weathering profiles through time: New insights from uranium-series isotopes. Earth and Planetary Science Letters, Vol. 274: 359-371.

Edwards, R., Gallup, C., and Cheng, H. 2003. Uranium-series dating of marine and lacustrine carbonates. In: Bourdon, B. et al. (eds.) Uranium-Series Geochemistry. Reviews in Mineralogy and Geochemistry, Vol. 52:363-406.

Fleitmann, D., Burns, S.J., Neff, U., Mangini, A., and Matter, A. 2003. Changing moisture sources over the last 330,000 years in Northern Oman from fluid inclusion evidence in speleothems. Quaternary Research, Vol. 60: 223-232.

Fontes, J.-C., Andrews, J.N., Causse, C., and Gibert, E. 1992. A comparison of radiocarbon and U/Th ages on continental carbonates. Radiocarbon, Vol. 34, No. 3: $602-610$.

Gascoyne, M. 1992. Geochemistry of the actinides and their daughters. In: Ivanovich, M. and Harmon, R.S. (eds.) Uranium-series Disequilibria. Oxford: Claredon Press, 34-61.

Ghaleb, B., Hillaire-Marcel, C., Causse, C., Gariepy, C., and Vallieres, S. 1990. Fractionation and recycling of $\mathrm{U}$ and $\mathrm{Th}$ isotopes in a semi-arid endoreic depression of central Syria. Geochimica et Cosmochimica Acta, Vol. 54: 1025 1035 .

Glennie, K.W., Boeuf, M.G.A., Hughes-Clark, M.W., Moody-Stuart, M., Pilaar, W.F.H., and Reinhardt, B.M. 1973.Late Cretaceous nappes in the Oman mountains and their geological evolution. American Association of Petroleum Geologists Bulletin, Vol. 57: 5-27. 
Glennie, K.W., Boeuf, M.G.A., Hughes-Clark, M.W., Moody-Stuart, M., Pilaar, W.F.H., and Reinhardt, B.M. 1974. Geology of the Oman Mountains. Part I: Text. Part II: Tables and Illustrations. Part III: Enclosures. Verhandelingen van het Koniklijk Nederlands geologisch mijnbouwkundig Genootschap, Vol. 31, pp. 423.

Govindaraju, K. 1994. 1994 Compilation of working values and sample description for 383 geostandards. Geostandards Newsletter, Vol. 18, Special Issue, pp. 158.

Haase-Schramm, A., Goldstein, S.L., and Stein, M. 2004. U-Th dating of Lake Lisan (late Pleistocene Dead Sea) aragonite and implications for glacial East Mediterranean climate change. Geochimica et Cosmochimica Acta, Vol. 68, No. 5: 985-1005.

Hacker, B.R., Mosenfelder, J.L., and Gnos, E. Rapid emplacement of the Oman ophiolite: Thermal and geochronologic constraints. Tectonics, Vol. 15, No. 6: 1230-1247.

Haggerty, J.A. 1991. Evidence from fluid seeps atop submarine seamounts in the Mariana forearc: clues for emplacement of the seamounts and their relationship go forearc tectonics. Marine Geology, Vol. 102: 293-309.

Hanghøj. K., Kelemen, P.B., Hassler, D., and Godard, M. 2010. Composition and genesis of depleted mantle peridotites from the Wadi Tayin Massif, Oman Ophiolite; Major and trace element geochemistry, and Os isotope and PGE systematics. Journal of Petrology, Vol. 51, No. 1-2: 201-227.

Hansen, R.O. and Stout, P.R. 1968. Isotopic distributions of uranium and thorium in soils. Soil Science, Vol. 105. No. 1: 44-50.

Hansen, R.O. 1970. Radioactivity of a California terrace soil. Soil Science, Vol. 110, No. 1: 31-36.

Harmon, S.R., Ford, D.C., and Schwarcz, H.P. 1977. Interglacial chronology of the Rocky and Mackenzie Mountains based upon ${ }^{230} \mathrm{Th}-{ }^{234} \mathrm{U}$ dating of calcite speleothems. Canadian Journal of Earth Sciences, Vol. 14: 2543-2552.

Kaufman, A. 1993. An evaluation of several methods for determining ${ }^{230} \mathrm{Th} / \mathrm{U}$ ages in impure carbonates. Geochimica et Cosmochimica Acta, Vol. 57: 2303-2317.

Kelemen, P.B. and Matter, J.M. 2008. In situ carbonation of peridotite for $\mathrm{CO}_{2}$ storage. Proceedings of the National Academy of Sciences, Vol. 105, No.45: 17295-17300.

Kelemen, P.B., Matter, J.M., Streit, E.E., Rudge, J.F., Curry, W.B., and Blusztajn, J. 2011. Rates and mechanisms of mineral carbonation in peridotite: Natural 
processes and recipes for enhanced, in situ $\mathrm{CO}_{2}$ capture and storage. Annual Reviews of Earth and Planetary Sciences, Vol. 39: 545-576.

Kelley, D.S., Karson, J.A., Früh-Green, G.L., Yoerger, D.R., Shank, T.M., Butterfield, D.A., Hayes, J.M., Schrenk, M.O., Olson, E.J., Proskurowski, G., Jakuba, M., Bradley, A., Larson, B., Ludwig, K., Glickson, D., Buckman, K., Bradley, A.S., Brazelton, W.J., Roe, K., Elend, M.J., Delacour, A., Bernasconi, S.M., Lilley, M.D., Baross, J.A., Summons, R.E., and Sylvia, S. P. 2005. A serpentinite-hosted ecosystem: The Lost City hydrothermal field. Science, Vol. 307: 1428-1434.

Krishnaswami, S., Williams, G.A., Graustein, W.C., and Turekian, K.K. 2004. The effect of weathering regime on uranium decay series and osmium in two soil profiles. Geochemical Journal, Vol. 38: 651-660.

$\mathrm{Ku}$, T.-L. and Liang, Z.-C. 1984. The dating of impure carbonates with decay-series isotopes. Nuclear Instruments and Methods in Physics Research, Vol. 223: 563 571.

Lackner, K.S., Wendt, C.H., Butt, D.P., Joyce, E.L., and Sharp, D.H. 1995. Carbon dioxide disposal in carbonate minerals. Energy, Vol. 20: 1153-1170.

Lang, S.Q., Butterfield, D.A., Schulte, M.D., Kelley, D.S. and Lilley, M.D. 2010. Elevated concentrations of formate, acetate, and dissolved organic carbon found at the Lost City hydrothermal field. Geochimica et Cosmochimica Acta, Vol. 74: 941-952.

Lao, Y. and Benson, L. 1988. Uranium-series age estimates ad paleoclimatic significance of Pleistocene tufas from the Lahotan basin, California and Nevada. Quaternary Research, Vol. 30: 165-176.

Lippard, S.J., Shelton, A.W., and Gass, I.G. 1986. The Ophiolite of Northern Oman. The Geological Society, Memoir No. 11. London: Blackwell Scientific Publications, pp. 178.

Lively, R.S., Harmon, R.S., Levinson, A.A., and Bland, C.J. 1979. Disequilibrium in the ${ }^{238}$ Uranium series in samples from Yeelirrie, Western Australia. Journal of Geochemical Exploration, Vol. 12: 57-65.

Ludwig, K.A. 2008. Formation, evolution, and age of the Lost City hydrothermal field. PhD Thesis. University of Washington, pp. 194

Ludwig, K.A., Shen, C.-C., Kelley, D.S., Cheng, H., and Edwards, L.R. 2011. U-Th systematic and ${ }^{230} \mathrm{Th}$ ages of carbonate chimneys at the Lost City hydrothermal field. Geochmica et Cosmochimica Acta, Vol. 75: 1869-1888. 
Ludwig, K.R. and Titterington, D.M. 1994. Calculation of ${ }^{230} \mathrm{Th} / \mathrm{U}$ isochrones, ages, and errors. Geochimica et Cosmochimica Acta, Vol. 58, No. 22: 5031-5042.

Ludwig, K.R. 2003. Mathematical-statistical treatment of data and errors for ${ }^{230} \mathrm{Th} / \mathrm{U}$ geochronology. In: Bourdon, B. et al. (eds.) Uranium-Series Geochemistry. Reviews in Mineralogy and Geochemistry, Vol. 52: 631-656.

Luo, S. and Ku, T-L. 1991. U-series isochron dating: A generalized method employing total sample dissolution. Geochimica et Cosmochimica Acta, Vol. 55: 555-564.

Ma, L., Chabaux, F., Pelt, E., Blaes, E., Jin, L., and Brantley, S. 2010. Regolith production rates calculated with uranium-series isotopes at Susquehanna/Shale Hills Critical Zone Observatory. Earth and Planetary Science Letters, Vol. 297: 211-225.

Mathieu, D., Bernat, M., and Nahon, D. 1995. Short-lived U and Th isotope distribution in a tropical laterite derived from granite (Pitinga river basin, Amazonia, Brazil): Application to assessment of weathering rate. Earth and Planetary Science Letters, Vol. 136: 703-714.

Neal, C. and Stanger, G. 1983. Hydrogen generation from mantle source rocks in Oman. Earth and Planetary Science Letters, Vol. 66: 315-320.

Neal, C. and Stanger, G. 1984. Calcium and magnesium-hydroxide precipitation from alkaline groundwaters in Oman, and their significance to the process of serpentinization. Mineralogical Magazine, vol. 48: 237-241.

Neal, C. and Stanger, G. 1985. Past and present serpentinization of ultramafic rocks: An example from the Semail ophiolite nappe of northern Oman. In: Drewer, J.I. (ed.), The Chemistry of Weathering. Holland: D. Reidel Publishing Company: 249-275.

Nicolas, A. 1989. Structures of ophiolites and dynamics of oceanic lithosphere. Amsterdam: Kluwer Academic Publishers, pp. 367.

Nicolas, A., Boudier, F., Ildefonse, V., and Ball, E. 2000. Accretion of Oman and United Arab Emirates ophiolite-Discussion of a new structural map. Marine Geophysical Researches, Vol. 21: 147-149.

Osmond, J.K., May, J.P., and Tanner, W.F. 1970. Age of the Cape Kennedy Barrier-and Lagoon Complex. Journal of Geophysical Research, Vol. 75, No. 2: 469-479.

Osmond, J.K. and Coward, J.P. 1992. Groundwater. In: Ivanovich, M. and Harmon, R.S. (eds.) Uranium-series Disequilibria. Oxford: Claredon Press, 290-334. 
Pearce, J.A., Alabaster, T., Shelton, A.W., and Searle, M.P. 1981. The Oman ophiolite as a Cretaceous arc-basin complex: Evidence and implications. Philosophical Transactions of the Royal Society of London, Series A: Mathematical and Physical Sciences, Vol. 300, No. 1454: 299-317.

Pelt, E., Chabaux, F., Innocent, C., Navarre-Sitchler, A.K., Sak, P.B., and Brantley, S.L. 2008. Uranium-thorium chronometry of weathering rinds: Rock alteration rate and paleoisotopic record of weathering fluids. Earth and Planetary Science Letters, Vol. 276: 98105.

Peretti, A., Dubessy, J., Mullis, J., Frost, B.R., and Tromsdorff, V. 1992. Highly reducing conditions during alpine metamorphism of the Malenco peridotite (Sondiro, northern Italy) indicated by mineral paragenesis and $\mathrm{H}_{2}$ in fluid inclusions. Contributions to Mineralogy and Petrology, Vol. 112: 329-340.

Porcelli, D. and Swarzenski, P.W. 2003. The behavior of U- and Th-series nuclides in groundwater. In: Bourdon, B. et al. (eds.) Uranium-Series Geochemistry. Reviews in Mineralogy and Geochemistry, Vol. 52: 363-406.

Przybylowicz, W., Schwarcz, H.P., and Latham, A.G. 1991. Dirty calcites 2. Uranium series dating of artificial calcite-detritus mixtures. Chemical Geology (Isotope Geoscience Section), Vol. 86: 161-178.

Robinson, C.S. and Rosholt, J.N. 1961. Uranium migration and geochemistry of uranium deposits in sandstone above, at, and below the water table. Part II: Relationship of uranium migration dates, geology, and chemistry of the uranium deposits. Economic Geology, Vol. 56: 1404-1420.

Rosholt, J.N. 1961a. Late Pleistocene and recent accumulation of uranium in ground water saturated sandstone deposits. Economic Geology, Vol. 56: 423-430.

Rosholt, J.N. 1961b. Uranium migration and geochemistry of uranium deposits in sandstone above, at, and below the water table. Part I: Calculation of apparent dates of uranium migration in deposits above and at the water table. Economic Geology, Vol. 56: 1392-1403.

Rosholt, J.N., Harshman, E.N., Shields, W.R., and Garner, E.L. 1964. Isotopic fractionation of uranium related to roll features in sandstone, Shirley Basin, Wyoming. Economic Geology, Vol. 59: 570-585.

Rosholt, J.N., Butler, A.P., Garner, E.L., and Shields, W.R. 1965a. Isotopic fractionation of uranium in sandstone, Powder River Basin, Wyoming and Slick Rock District, Colorado. Economic Geology, Vol. 60: 199-213. 
Rosholt, J.N., Tatsumoto, M., and Dooley, J.R. 1965b. Radioactive disequilibrium studies in sandstone, Powder River Basin, Wyoming, and Slick Rock District, Colorado. Economic Geology, Vol. 60: 477-484.

Rosholt, J.N., Doe, B.R., and Tatsumoto, M. 1966. Evolution of the isotopic composition of uranium and thorium in soil profiles. Geological Society of America Bulletin, Vol. 77: 987-1004.

Rosholt, J.N. 1976. ${ }^{230} \mathrm{Th} /{ }^{234} \mathrm{U}$ dating of travertine and caliche rinds. Geological Society of America Abstracts, Program 8: 1076.

Rosholt, J.N., Bush, C.A., Shroba, R.R., Pierce, K.L., and Richmond, G.M. 1985. Uranium-trenddating and calibrations for Quaternary sediments. Open-File Report 85-299, U.S. Geological Survey, Lakewood, Colorado, pp. 48

Schwarcz, H.P. and Latham, A.G. 1989. Dirty calcites 1. Uranium-series dating of contaminated calcite using leachates alone. Chemical Geology (Isotope Geoscience Section), Vol. 80:35-42.

Searle, M.P. and Cox, J. 1999. Tectonic setting, origin, and obduction of the Oman ophiolite. Geological Society of America Bulletin, Vol. 111, No. 1: 104-122.

Sims, K.W.W., Hart, S.R., Reagen, M.K., Blusztajn, J., Staudigel, H., Sohn, R.A., Layne, G.D., Ball, L.A., and Andrews, J. 2008a. ${ }^{238} \mathrm{U}^{2}{ }^{230} \mathrm{Th}^{226} \mathrm{Ra}^{210} \mathrm{~Pb}^{210}{ }^{21} \mathrm{Po},{ }^{232} \mathrm{Th}$ ${ }^{228} \mathrm{Ra}$, and ${ }^{235} \mathrm{U}-{ }^{231} \mathrm{~Pa}$ constraints on the ages and petrogenesis of Vailulu'u and Malumalu Lavas, Samoa. Geochemistry, Geophysics, Geosystems, Vol. 9, No. 4: pp 30.

Sims, K.W.W., Gill, J.B., Dosseto, A., Hoffman,D.L., Lundstrom, C.C., Williams, R.W., Ball, L., Tollstrup, D., Turner, S., Prytulak, J., Glessner, J. J.G., Standish, J.J., and Elliot, T. 2008b. Geostandards and Geoanalytical Research, Vol. 32, No. 1: 65 91.

Szabo, B.J. and Rosholt, J.N. 1982. Surficial continental sediments. In: Ivanovich, M. and Harmon, R.S. (eds.) Uranium Series Disequilibrium: Applications to Environmental Problems. London: Clarendon Press, 257-260.

Thompson, W.G., Spiegelman, M.W., Goldstein, S.L., and Speed, R.C. 2003. An open system model for U-series age determinations of fossil corals. Earth and Planetary Science Letters, Vol. 210: 365-381.

Villemant, B. and Feuillet, N. 2003. Dating open systems by the ${ }^{238} \mathrm{U}_{-}^{234} \mathrm{U}_{-}{ }^{230}$ Th method: 
application to Quaternary reef terraces. Earth and Planetary Science Letters, Vol. 210: 105-118.

Wilson, S.A., Raudseep, M., and Dipple, G.M. 2006. Verifying and quantifying carbon fixation in minerals from serpentine-rich mine tailings using the Rietveld method with X-ray powder diffraction data. American Mineralogist, Vol. 91, No. 8-9: 1331-1341.

Wilson, S.A., Raudsepp, M., and Dipple, G.M. 2009a. Quantifying carbon fixation in trace minerals from processed kimberlite: A comparative study of quantitative methods using X-ray powder diffraction data with applications to the Diavik Diamond Mine, Northwest Territories, Canada. Applied Geochemistry, Vol. 24, No. 12: 95-112.

Wilson, S.A., Dipple, G.M., Power, I.M., Thom, J.M., Anderson, R.G., Raudsepp, M., Gabites, J.E., and Southam, G. 2009b. Carbon dioxide fixation within mine wastes of ultramafic-hosted ore deposits: Examples from the Clinton Creek and Cassiar chrysotile deposits, Canada. Economic Geology, Vol. 104, No. 1: 95-112. 


\title{
Chapter 4: ${ }^{3}$ He Exposures Ages and Erosion Rates of Serpentinized Peridotite Bedrock, Samail Ophiolite, Sultanate of Oman
}

\begin{abstract}
:
Understanding timescales of weathering and erosion of ultramafic rocks is important in order to evaluate the role of ultramafic rocks, such as peridotite, in various geological processes and cycles, such as the global carbon cycle. However, ultramafic erosion rates are poorly constrained. To investigate rates of erosion of subaerial peridotite in an arid environment, helium isotopes were measured in partially-serpentinized peridotite bedrock from the Samail Ophiolite, Sultanate of Oman. Cosmogenic ${ }^{3} \mathrm{He}$ was detected in several samples, allowing the calculation of minimum exposure ages and maximum erosion rates for peridotite bedrock sampled at three locations (Qafeefah, Misht, and Wadi Sudari) in the ophiolite. The minimum exposure ages range from $\sim 3,000$ to $91,000 \mathrm{yrs}$, and the maximum erosion rates range from $\sim 5$ to $180 \mathrm{~m} / \mathrm{Myr}$. The maximum erosion rates suggest that at most $\sim 10^{5}-10^{6} \mathrm{~kg} \mathrm{CO}_{2} / \mathrm{yr}$ is lost through erosion of peridotite bedrock in the Samail Ophiolite. However, the fate of this $\mathrm{CO}_{2}-$ whether it continues to be stored in carbonate-rich cements in peridotite conglomerates or in surrounding alluvial fans, or whether significant amounts are released to the hydrosphere and/or atmosphere - remains an open question.
\end{abstract}

\section{Introduction:}

Ultramafic rocks, which are primarily composed of olivine and pyroxene, are expected to weather and erode more quickly than other types of igneous rocks, such as mafic basalts. Olivine and pyroxene are far from equilibrium with $\mathrm{H}_{2} \mathrm{O}$ and $\mathrm{CO}_{2}$ on the Earth's surface and thus easily altered to hydrous silicates, Fe-oxides, and carbonates 
(calcite, magnesite, dolomite) (e.g. Barnes et al., 1967, 1978; Barnes and O’Neil, 1969; Evans, 1977; Trommsdorff and Evans, 1977; Trommsdorff et al., 1980; Frost, 1985; Kelemen et al., 2011). Furthermore, olivine and pyroxene dissolution rates are significantly faster than those of other silicate minerals (e.g. Luce et al., 1972; Van Herk et al., 1989; Wogelius and Walther, 1991; Chen and Brantley, 2000; Pokrovski and Schott, 2000; Giammar et al., 2004; Hänchen et al., 2006; Prigiobbe et al., 2009).

While subaerial exposures of ultramafic bedrock are small in volume relative to other types of igneous rocks, determining erosion rates of ultramafic rocks is important for a number of reasons. Firstly, even small volumes of ultramafic bedrock may have a significant influence on various geological processes and cycles due to the expected fast weathering and erosion rates for ultramafic rocks. Secondly, erosion rates are important to consider when investigating timescales of natural carbonation of ultramafic rocks such as peridotite (Chapters 2 and 3; also Wilson et al., 2006, 2009a, b; Kelemen and Matter, 2008; Matter and Kelemen, 2009; Kelemen et al., 2011) and in evaluating the viability of using artificially-enhanced, in situ alteration of peridotite to mitigate the buildup of anthropogenic $\mathrm{CO}_{2}$ input to the atmosphere (Seifritz, 1990; Lackner et al., 1995; Kelemen and Matter, 2008; Matter and Kelemen, 2009; Kelemen et al., 2011). Ultramafic weathering and erosion rates are also important to consider when investigating the development of economic deposits of metals such as nickel, platinum, chromium, cobalt, and manganese in soils derived from ultramafic rocks (e.g. Golightly, 1981, 2010; Summons et al., 1981; Llorca and Monchoux, 1991; Cook and Fletcher, 1993; Gleeson et al., 2003; Dzemua and Gleeson, 2012). Finally, weathering and erosion of ultramafic 
rocks is locally important in regions with large exposures of ultramafic rocks, such as the mantle peridotite layer of ophiolites (e.g. Oman and the United Arab Emirates, Papua New Guinea, New Caledonia, Cyprus, the Alps, and the western USA), komatiites (e.g. South Africa, Australia, Canada), and kimberlites (e.g. South Africa, Tanzania, Canada).

Despite the potential importance of ultramafic erosion rates for various geological processes and cycles, only a few studies have investigated rates of erosion of ultramafic rocks (e.g. Moretti and Turcotte, 1985; Freyssinet and Farah, 2000; Beuvais et al., 2007; Hanson et al., 2009). This is because ultramafic rocks are generally significantly altered making determination of erosion rates using quantitative methods, such as measurement of cosmogenic nuclides, highly challenging.

Cosmogenic nuclides are produced in the top $\sim 2$ meters of continental crust, dominantly by neutron-induced spallation, and can be powerful tools for studying erosion on different timescales (e.g. Lal, 1991; Kurz et al., 1986a, b; Kurz et al., 1990; Kurz and Brooks, 1994; Portenga and Bierman, 2011). Erosion rates have been determined for a variety of rock types using cosmogenic nuclides such as ${ }^{10} \mathrm{Be}$ (e.g. Portenga and Bierman, 2011) and ${ }^{3} \mathrm{He}$ (e.g. Kurz, 1986a, b; Kurz et al., 1990). However, measurement of cosmogenic nuclides in ultramafic bedrock has not been extensively exploited. Quartz, the most commonly analyzed mineral for ${ }^{10} \mathrm{Be}$, is rare in ultramafic rocks, and ${ }^{10} \mathrm{Be}$ measurements are difficult in olivine (e.g. Blard et al., 2008). Cosmogenic ${ }^{3} \mathrm{He}$ is generally measured in olivine and pyroxene, which are often highly altered in ultramafic bedrock. 
Cosmogenic ${ }^{3} \mathrm{He}$ has been extensively measured in basaltic olivine and pyroxene phenocrysts (e.g. Kurz, 1986b; Kurz et al., 2004). While pristine olivine and pyroxene can be fairly easily obtained from mafic rocks, such as basaltic lava flows (e.g. Kurz, 1986a, b; Kurz et al., 1990; Kurz et al., 2004), and also from ultramafic xenoliths (e.g. Porcelli et al., 1987; Yokochi et al., 2005; Moreira and Madureira, 2005; Aka et al., 2009; Anslow et al., 2010), ultramafic bedrock is commonly highly altered and lacks pristine olivine and pyroxene. There are very limited numbers of helium gas studies for in situ deep crust or mantle rocks. However, an initial study on two abyssal peridotites by Kumagai et al. (2003) demonstrated that despite the relatively high degree of alteration ( $\sim 85 \%$ of the olivine is weathered to brown-colored clays and other secondary minerals) of these peridotites, clinopyroxene mineral separates retained mantle-like helium signatures $\left({ }^{3} \mathrm{He} /{ }^{4} \mathrm{He}=8.30\right.$ and $\left.8.13 \mathrm{R} / \mathrm{R}^{\mathrm{A}}\right)$ and had much higher He concentrations (158.7 ncc/g and $30.7 \mathrm{ncc} / \mathrm{g}$ ) than expected. Similarly, Kurz et al. (2009) demonstrated that whole rock fractions from submarine and subaerial mylonites (deformed peridotites), a protomylonitic peridotite, and a protogranular peridotite have mantle-like ${ }^{3} \mathrm{He} /{ }^{4} \mathrm{He}$ (generally $\sim 6-10 \mathrm{R} / \mathrm{R}_{\mathrm{A}}$; one dunite mylonite had a lower value of $2.23 \mathrm{R} / \mathrm{R}_{\mathrm{A}}$ ) and variable (0.70-6105 ncc/g) but generally high He concentrations.

Furthermore, preliminary measurements of cosmogenic ${ }^{3} \mathrm{He}$ in peridotite bedrock from the Josephine Ophiolite indicate that obtaining ${ }^{3} \mathrm{He}$ exposure ages and erosion rates for partially-serpentinized peridotite bedrock is feasible (Recanati et al., submitted). However, He may exhibit very different behavior in altered ultramafic rocks relative to 
young, pristine basalts, and hence investigation of this behavior will be an important step in developing cosmogenic ${ }^{3} \mathrm{He}$ dating for ultramafic rocks.

The goal of this study is to measure helium in peridoite bedrock from the Samail Ophiolite, Sultanate of Oman, in order to evaluate the use of cosmogenic ${ }^{3} \mathrm{He}$ for determining exposure ages and erosion rates. Because we are specifically interested in determining rates of erosion for altered (partially-serpentinized and carbonated) ultramafic rocks, the peridotites analyzed in this study are $\sim 30-90 \%$ serpentinized. Analysis of both mineral separates and whole rock fractions also permits us to investigate the behavior of He during serpentinization of peridotite.

\section{Geologic Setting:}

The Samail Ophilote (Figure 1) is located in the Sultanate of Oman and the United Arab Emirates and is one of the largest and best-exposed ophiolites in the world. The ophiolite consists of Cretaceous oceanic crust $(4-7 \mathrm{~km})$ and mantle $(8-12 \mathrm{~km})$ that was uplifted and emplaced along with the Hawasina pelagic sedimentary formation (Glennie et al., 1973, 1974; Coleman, 1981; Lippard et al., 1986; Nicolas et al., 2000). The ophiolite is approximately $50-100 \mathrm{~km}$ in width and extends for greater than $500 \mathrm{~km}$ in length (Lippard et al., 1986; Nicolas et al., 2000). The mantle layer of the Samail Ophiolite consists of peridotites which are variably serpentinized and tectonized (e.g. Lippard et al., 1986). Harzburgites, which locally grade into lherzolites, comprise $\sim 85$ $95 \%$ of the mantle sequence of the Samail Ophioite (e.g. Lippard et al., 1986). Light brown dunite bodies, which are easily distinguished from the darker harzburgites, comprise $\sim 5-15 \%$ of the mantle layer (e.g. Lippard et al., 1986). Veins and bodies of 
other ultramafic rocks, such as pyroxenite, are also found in the mantle layer but are volumetrically insignificant. Generally, $50-80 \%$ of the primary silicate minerals in the peridotite have been altered to secondary minerals such as lizardite, chrysotile, carbonates, and magnetite (e.g. Lippard et al., 1986).

A more detailed discussion of the geology of the Samail Ophiolite is presented in Chapter 2 and also in the summaries found in Glennie et al. (1973, 1974), Coleman (1981), Pearce et al. (1981), Lippard et al. (1986), Nicolas (1989), Hacker et al. (1996), Searle and Cox (1999), and Nicolas et al. (2000).

\section{Sample Descriptions:}

Altered peridotites were collected from seven locations in the peridotite layer of the Samail Ophiolite (Figure 1): four locations of travertine deposition (Faleej Travertine, Qafeefah Travertine, and Misht Travertine), two roadcuts exposing abundant carbonate and serpentine veins (Qafeefah Roadcut and Fanja Roadcut), and one campsite located on the natural peridotite weathering surface and containing abundant carbonate veins (Wadi Sudari Campsite). Note that Qafeefah Roadcut and Wadi Sudari Campsite are located very close (within 2-3 km) to Qafeefah Travertine and Wadi Sudari Travertine, respectively. Therefore, in this paper the samples from Qafeefah Travertine and Roadcut as well as Wadi Sudari Travertine and Campsite are considered together. A list of samples with UTM coordinates is given in Table 1, and sample descriptions for each location are given below.

With the exception of Faleej where a single unshielded sample was collected, at each field location 3 to 4 individual rock samples were selected for helium analysis. One 
sample from each location was collected from a fully or partially shielded location while the remaining samples were collected from locations with minimal modern day shielding from cosmic rays. Since cosmogenic production of ${ }^{3} \mathrm{He}$ occurs primarily in the top $\sim 2 \mathrm{~m}$ and decreases exponentially with depth (e.g. Kurz, 1986b), samples collected deeper than $\sim 2 \mathrm{~m}$ from the current exposed surface should contain minimal cosmogenic ${ }^{3} \mathrm{He}$. Shielded samples are useful because the amount of ${ }^{3} \mathrm{He}$ contributed by mantle, radiogenic, and atmospheric sources is uncertain, particularly for ophiolite terrains where the helium systematics have not been well-constrained. Therefore, shielded samples are useful to employ for corrections in similar unshielded samples of ${ }^{3} \mathrm{He}$ contributed by noncosmogenic sources. Shielded samples were collected from roadcut surfaces and also from underneath $\sim 2 \mathrm{~m}$ thick travertine terraces.

Faleej:

At Faleej Travertine (Figure 2), one peridotite sample (OM09-2P-MS) was collected in 2009 as part of an initial feasibility study. This sample is a peridotite cobble (Figure 2B) that was found interbedded with travertine at the top of travertine terrace.

\section{Fanja:}

Three peridotite samples were collected at Fanja Roadcut (Figure 3). Two samples (OM10-2P-MG and OM10-3P-MG) were collected above the roadcut from the natural peridotite weathering surface (Figure 3A). The third sample (OM10-4P-MG) was collected from the bottom of a $\sim 5 \mathrm{~m}$ tall roadcut face (Figure 3B). Due to its location on a 
vertical face and also the young age of the roadcut ${ }^{1}$, OM10-4P-MG has likely not experienced significant exposure to cosmic rays.

\section{Qafeefah:}

Four samples were collected at Qafeefah (Figure 4). Three of these samples were collected on the natural peridotite weathering surface exposed at the large travertine deposit at Qafeefah (Figure 4A; see Chapters 2 and 3 for a description of the travertine). Samples OM09-4P-MS and OM10-4P-MG were collected in the valley that contains the travertine deposit. Sample OM10-11P-MG was collected from the top of small peridotite hill adjacent to the valley (Figure $4 \mathrm{~A}$ ). Approximately $2-3 \mathrm{~km}$ from the primary travertine deposit at Qafeefah there is a large roadcut exposure along the mountain pass to Wadi Dima (Figure 4B). Sample OM10-10P-MG was collected from the bottom of a $\sim 10 \mathrm{~m}$ roadcut exposure at Qafeefah Roadcut (Figure 4B). Similar to Sample OM10-4P-MG from Fanja Roadcut, this sample experienced minimal cosmic ray exposure.

Misht:

Three samples were collected at Misht Travertine (Figure 5). Two of the samples (OM10-14P-MG and OM10-17P-MG) were collected from the natural peridotite weathering surface. Sample OM10-14P-MG was collected from the top of a peridotite hill adjacent to the travertine terraces at the northwest end of the travertine deposit (Figure 5A; see Chapters 2 and 3 for a description of the travertine). Sample OM10-17PMG was collected from a valley at the opposite end of the $\sim 2 \mathrm{~km}$ long travertine deposit

\footnotetext{
${ }^{1}$ Fanja Roadcut and also Qafeefah Roadcut were likely constructed within the last 5-10 years. Virtually all roadcuts in the Sultanate of Oman have been constructed within the last 40 years. These timescales of exposure are insignificant relative to the $\sim 3,000-91,000 \mathrm{yr}$ exposure ages of Samail peridotites.
} 
(Figure 5B). Sample OM10-17P-MG was collected in the vicinity of the warm $\left(\sim 35^{\circ} \mathrm{C}\right)$ alkaline pools which are used for bathing by the residents of the nearby village of AlBana. Sample OM10-13P-MG (Figure 5C) was collected from highly-altered peridotite bedrock exposed underneath a $\sim 2 \mathrm{~m}$ thick travertine terrace that is $\sim 29,000$ to $\sim 45,000$ years in age based on ${ }^{14} \mathrm{C}$ dating (Chapter 2). Therefore, Sample OM10-13P-MG has been at least partially shielded from cosmic rays for the past $\sim 45,000$ years.

\section{Wadi Sudari:}

Three samples were collected at Wadi Sudari (Figure 6). Two of the samples (OM10-19P-MG and OM10-22P-MG) were collected from the natural peridotite weathering surface. Sample OM10-19P-MG was collected from a peridotite hill adjacent to the large travertine deposit at Wadi Sudari (Figure 6A; see Chapters 2 and 3 for a description of the travertine). Sample OM10-20P-MG was collected from a flat peridotite weathering surface located $\sim 2-3 \mathrm{~km}$ from the travertine deposit. This flat area was used as a campsite during our field campaigns and is referred to as "Wadi Sudari Campsite." Sample OM10-20P-MG was collected from the side of an incised channel in the Wadi Sudari travertine deposit. This incised channel cuts through both the peridotite bedrock and an overlying $\sim 2 \mathrm{~m}$ thick travertine terrace, both of which provide shielding for OM10-20P-MG (Figure 6B). The top of the travertine terrace has been dated to $\sim 34,000$ yr BP using ${ }^{14} \mathrm{C}$ dating (Chapter 2). 


\section{Methods:}

\subsection{Sample Preparation:}

Peridotites selected for helium analysis were hand-picked from a 0.85 to $2 \mathrm{~mm}$ size crushed, sieved samples. For select samples, magnetic separation was also employed prior to hand-picking. For the less-altered samples, olivine, altered olivine, and/or pyroxene mineral separates were obtained through hand-picking. For more altered samples, purified whole rock fractions were made by removing obvious large fragments of carbonate and serpentine and leaving behind partially-altered grains. Prior to analysis, the hand-picked grains were cleaned for 15 minutes in a sonicated acetone wash. Representative images of olivine, altered olivine, pyroxene, and whole rock grains are shown in Figure 7. Thin sections were prepared for all samples, and minerals were identified in whole rock powders using X-ray diffraction at Woods Hole Oceanographic Institution.

\section{2: Helium Measurements:}

All helium measurements were carried out at Woods Hole Oceanographic Institution using well-established methods (e.g. Kurz et al., 1986a, 1986b; Kurz et al., 1990; Kurz et al., 2004). The helium measurements were made on the MS2 mass spectrometer, which is an automated, dual collection, statically operated helium isotope mass spectrometer with a Nier-type ion source. Samples were first crushed in vacuo, and the powder remaining from the crush was heated at $1600^{\circ} \mathrm{C}$. Blanks were typically $3 \mathrm{x}$ $10^{-11}$ cc STP ${ }^{4} \mathrm{He}$. 


\section{Results and Discussion:}

\subsection{Mineralogy:}

The samples are variably serpentinized $(30-90 \%)$ peridotites. The three rocks from Fanja Roadcut are altered dunites while all other samples are altered harzburgites. Representative thin section pictures are given in Figure 8 which shows a moderately $(\sim 40 \%)$ serpentinized harzburgite (panels A and B), a highly serpentinized ( $90 \%)$ harzburgite (Panels $\mathrm{C}$ and $\mathrm{D})$, and a moderately serpentinized $(\sim 30 \%)$ dunite (Panels $\mathrm{E}$ and F).

Results of X-ray diffraction on whole rock powders are presented in Table 1. Mineral peaks were identified as "major" or "trace" but were not further quantified. The dominant mineral in all of the samples is serpentine, in the form of chrysotile for most samples and lizardite for samples OM09-4P-MS, OM10-09-MG, and OM10-19P-MG. Most samples also contain both olivine (forsterite) and pyroxene (enstatite, diopside). Many samples also contain other secondary alteration minerals such talc, hydrotalcite and carbonate. Samples OM09-2P-MG, OM10-20P-MG, and OM10-22P-MG are among the least altered and contain both forstertite and enstatite as major minerals. Samples OM102P-MG, OM10-3P-MG, and OM10-4P-MG, which are dunites, contain forsterite as a major mineral and do not contain significant pyroxene. Samples OM09-4P-MS and OM10-9P-MG are dominated by lizardite serpentine but contain olivine and pyroxene as 
trace minerals. Sample OM10-19P-MG is highly serpentinized and consists of $\sim 100 \%$ lizardite with no significant olivine or pyroxene.

\subsection{Helium Measurements:}

The results of helium concentration and isotopic measurements are presented in Table 2. Figures 9 through 13 display plots of ${ }^{4} \mathrm{He}(\mathrm{cc} / \mathrm{g}),{ }^{3} \mathrm{He}(\mathrm{cc} / \mathrm{g})$, and ${ }^{3} \mathrm{He} /{ }^{4} \mathrm{He}\left(\mathrm{R} / \mathrm{R}_{\mathrm{A}}\right)$ for each site. Crush values are indicated by open symbols while melt values are indicated by closed symbols. Although all samples may have experienced some shielding and the shielded samples may not have been completely shielded, the samples from roadcuts and from underneath thick travertine terraces are referred to as "shielded" while all other samples are referred to as "unshielded." Unshielded samples are indicated by colored symbols while shielded samples are indicated by black symbols.

\subsection{1 ${ }^{4} \mathrm{He}$ Concentrations:}

${ }^{4} \mathrm{He}$ concentrations range from $\sim 8 \times 10^{-11}$ to $\sim 2 \times 10^{-7} \mathrm{cc} \mathrm{STP} / \mathrm{g}$ for measurements by crushing in vacuo and from $\sim 2 \times 10^{-9}$ to $\sim 1 \times 10^{-6} \mathrm{cc} \mathrm{STP} / \mathrm{g}$ for melting. These concentrations are lower than those typically observed in MORB glasses (e.g. Staudacher et al., 1989; Moreira et al., 1998) but are similar to those observed in peridotite xenoliths, seafloor peridotites, and ophiolite peridotites (e.g. Gautheron et al., 2005; Kurz et al., 2009; Recanati et al., submitted). Samples OM10-2P-MG and OM10-4P-MG (both from Fanja) and samples OM10-20P-MG (whole rock fraction only) and OM10-22P-MG (both from Wadi Sudari) have notably higher ${ }^{4} \mathrm{He}$ concentrations than the other samples. Samples OM10-20P-MG and OM10-22P-MG are among the least altered, which may explain their higher ${ }^{4} \mathrm{He}$ concentrations if $\mathrm{He}$ is being lost via alteration of the peridotites. 
However, samples OM10-2P-MG and OM10-4P-MG are among the most altered, so the enriched ${ }^{4} \mathrm{He}$ concentrations of these samples is perhaps due to regional variations in $\mathrm{He}$ distribution.

For all samples, the melt fractions have higher He concentrations than the corresponding crush values. This is not surprising since crushing is thought to selectively release magmatic ${ }^{4} \mathrm{He}$ (e.g. Kurz et al., 1986a, 1986b) while melting releases a combination of magmatic and radiogenic ${ }^{4} \mathrm{He}$. Since the Samail peridotites formed $\sim 95$ Ma (e.g. Tilton et al., 1981; Rioux et al., 2008), they likely contain significant radiogenic ${ }^{4}$ He. With the exception of Sample OM10-20P-MG, which is the least altered of all the samples, for a given rock sample, olivine and pyroxene separates have higher ${ }^{4} \mathrm{He}$ concentrations than the corresponding whole rock fractions. However, at some locations - most notably Fanja Roadcut and Wadi Sudari- whole rock fractions from one rock have higher ${ }^{4} \mathrm{He}$ concentrations than mineral separates from another rock, which indicates that there is likely small-scale heterogeneity in He concentration. The whole rock separates contain significant serpentine. There is only one prior helium analysis of serpentine in the literature, for a serpentine vein in oceanic peridotite from the Southwest Indian Ridge (Kurz et al., 2009). This serpentine vein had total (crush + melt) ${ }^{4} \mathrm{He}=6.86$ $\mathrm{x} 10^{-9} \mathrm{cc} \mathrm{STP} / \mathrm{g}$, which is at the low end of the range of the ${ }^{4} \mathrm{He}$ concentrations measured in Samail whole rock fractions.

\subsubsection{He Isotopes:}

${ }^{3} \mathrm{He} /{ }^{4} \mathrm{He}$ values range from 0.21 to $8.41 \mathrm{R} / \mathrm{R}_{\mathrm{A}}$ for crush analyses and from 0.35 to 218.98 R/R $\mathrm{R}_{\mathrm{A}}$ for melt fractions (Table 2). The variable ${ }^{3} \mathrm{He} /{ }^{4} \mathrm{He}$ values observed in the 
Samail Ophiolite peridotites are very different from the results of the only previous study of ophiolite peridotite in the $\sim 150$ Ma Josepehine Ophiolite by Recanati et al. (submitted), who observed a narrow range of total ${ }^{3} \mathrm{He} /{ }^{4} \mathrm{He}=6.7 \pm 0.2 \mathrm{R} / \mathrm{R}_{\mathrm{A}}(\mathrm{n}=33)$ in olivine, pyroxene, and whole rock fractions. The peridotites analyzed by Recanati et al. are less altered than the partially-serpentinized peridotites analyzed in this study. The wide range of ${ }^{3} \mathrm{He} /{ }^{4} \mathrm{He}$ values observed in the Samail peridotites indicates mixing of mantle, radiogenic (see discussion below in Section 5.3), atmospheric, and cosmogenic He.

In basaltic phenocrysts, the crush fraction preferentially releases magmatic $\mathrm{He}$ from fluid and melt inclusions (e.g. Kurz et al., 1986b). However, most of the crush fractions in the Samail peridotites have significantly lower isotopic values than those observed in mid-ocean ridge basalts (MORB), which have ${ }^{3} \mathrm{He} /{ }^{4} \mathrm{He}=8 \pm 1 \mathrm{R} / \mathrm{R}_{\mathrm{A}}$ (e.g. Allégre et al., 1995; Farley and Neroda, 1998). Only samples OM10-2P-MG $\left({ }^{3} \mathrm{He} /{ }^{4} \mathrm{He}=\right.$ 7.83 R/R $\left.\mathrm{R}_{\mathrm{A}}\right)$ and OM10-4P-MG $\left({ }^{3} \mathrm{He} /{ }^{4} \mathrm{He}=8.41 \mathrm{R} / \mathrm{R}_{\mathrm{A}}\right.$ in the olivine fraction and ${ }^{3} \mathrm{He} /{ }^{4} \mathrm{He}$ $=7.93 \mathrm{R} / \mathrm{R}_{\mathrm{A}}$ in the whole rock fraction) have isotopic compositions typical of those observed in MORB. Samples OM10-3P-MG $\left({ }^{3} \mathrm{He} /{ }^{4} \mathrm{He}=5.91 \mathrm{R} / \mathrm{R}_{\mathrm{A}}\right)$ and OM10-20P-MG $\left({ }^{3} \mathrm{He} /{ }^{4} \mathrm{He}=6.33 \mathrm{R} / \mathrm{R}_{\mathrm{A}}\right.$ in the olivine fraction) have isotopic compositions more similar to those observed in ultramafic xenoliths and basalts sampled from continental areas, which have lower $\mathrm{R} / \mathrm{R}_{\mathrm{A}}=6.1 \pm 0.9$ (Gautheron and Moreira, 2002), and also in the Josephine Ophiolite peridotites by Recanati et al. (submitted). The remaining crush fractions have significantly lower $\mathrm{R} / \mathrm{R}_{\mathrm{A}}$ indicating the presence of atmospheric $\left({ }^{3} \mathrm{He} /{ }^{4} \mathrm{He}=1.0 \mathrm{R} / \mathrm{R}_{\mathrm{A}}\right)$ 
and radiogenic $\left(\mathrm{R} / \mathrm{R}_{\mathrm{A}}=\sim 0.01\right.$ to 1 ; e.g. ${ }^{3} \mathrm{He} /{ }^{4} \mathrm{He} \sim 0.01$ to $0.1 \mathrm{R} / \mathrm{R}_{\mathrm{A}}$; e.g. Morrison and Pine, 1955; Andrews, 1985) helium.

Shielded samples have $\mathrm{R} / \mathrm{R}_{\mathrm{A}}$ melt values that are similar to (OM10-4P-MG and OM10-20P-MG) or slightly higher (OM10-10-MG and OM10-13P-MG) than the corresponding crush fractions, which suggests that these samples do not contain significant cosmogenic ${ }^{3} \mathrm{He}$. The unshielded samples consistently have higher $\mathrm{R} / \mathrm{R}_{\mathrm{A}}$ in the melt fractions relative to the crush fractions, which suggests that these samples contain some cosmogenic ${ }^{3} \mathrm{He}$ since cosmogenic helium is preferentially released during melting (e.g. Kurz et al., 1986a, b). Sample OM09-4P-MS has $R / R_{A}=218$ in the melt fraction, which indicates unambiguous cosmogenic ${ }^{3} \mathrm{He}$. This value is more than 4 times the highest $R / R_{A}$ observed in the most primitive terrestrial reservoirs, which are $R / R_{A}=37$ for lavas associated with the Icelandic plume (Hilton et al., 1999) and $\mathrm{R} / \mathrm{R}_{\mathrm{A}}=50$ for $\sim 61$ Ma lavas from Baffin Island (Stuart et al., 2003). Since the Samail Ophiolite is not associated with a hotspot, there is no reason to believe that mantle $R / R_{A}$ values in Oman will differ significantly from the 6 to $9 \mathrm{R} / \mathrm{R}_{\mathrm{A}}$ range typically observed in ultramafic xenoliths and MORB. Therefore, sample O10-14P-MG $\left(\mathrm{R} / \mathrm{R}_{\mathrm{A}}=30\right.$ in a pyroxene melt fraction and $\mathrm{R} / \mathrm{R}_{\mathrm{A}}=19$ in a whole rock melt fraction) also likely contains significant cosmogenic ${ }^{3} \mathrm{He}$. Other unshielded samples do not have elevated $\mathrm{R} / \mathrm{R}_{\mathrm{A}}$ values indicating the clear presence of cosmogenic ${ }^{3} \mathrm{He}$. However, several samples from Qafeefah (OM109P-MG and OM10-11P-MG in addition to OM09-4P-MS) (Figure 11), Misht (O10-17PMG in addition to OM10-14P-MG) (Figure 12), and Wadi Sudari (OM10-22P-MG) (Figure 13) have elevated ${ }^{3} \mathrm{He}$ concentrations relative to the ${ }^{3} \mathrm{He}$ concentrations in 
shielded samples from the same locations. This may indicate the presence of cosmogenic ${ }^{3} \mathrm{He}$ in these samples. In contrast, unshielded samples OM10-2P-MG and OM10-4P-MG (from Fanja) and sample OM10-19P-MG (from Wadi Sudari) have lower ${ }^{3} \mathrm{He}$ concentrations than the associated shielded samples. Possible explanation for this in the Fanja samples are the presence of significant radiogenic ${ }^{4} \mathrm{He}$ diluting the cosmogenic ${ }^{3} \mathrm{He}$ signal, or loss of cosmogenic helium. The low value in OM10-19P-MG may be due to the almost complete serpentinization of this sample (see Section 5.1).

\subsection{Radiogenic Production of ${ }^{4} \mathrm{He}$ from $\mathrm{U}$ and Th Decay:}

The low ${ }^{3} \mathrm{He} /{ }^{4} \mathrm{He}$ values of several of the Samail peridotites suggest the presence of a significant radiogenic helium component $\left({ }^{3} \mathrm{He} /{ }^{4} \mathrm{He} \sim 0.01\right.$ to $0.1 \mathrm{R} / \mathrm{R}_{\mathrm{A}}$; e.g. Morrison and Pine, 1955; Andrews, 1985). Several samples have crush (OM09-4P-MS, OM1013P-MG, OM10-14P-MG, OM10-17P-MG, OM10-19P-MG, OM10-20P-MG, and OM10-22P-MG) and melt (OM10-13P-MG and OM10-20P-MG) ${ }^{3} \mathrm{He} /{ }^{4} \mathrm{He}$ values $<1$ $\mathrm{R} / \mathrm{R}_{\mathrm{A}}$ (i.e. lower than atmospheric value used for normalization), which indicates the unambiguous presence of radiogenic ${ }^{4} \mathrm{He}$. Furthermore, the presence of ${ }^{3} \mathrm{He} /{ }^{4} \mathrm{He}<1$ in crush fractions indicates that the radiogenic component is present not only in solid phases but also in phases (e.g. fluid or melt inclusions, fractures) released during crushing.

The presence of radiogenic helium in the Samail peridotites is not surprising considering the $\sim 95 \mathrm{Ma}$ age of the peridotites (e.g. Tilton et al., 1981; Rioux et al., 2008; Rioux et al., submitted, 2012). Radiogenic helium is primarily produced through decay of ${ }^{238} \mathrm{U},{ }^{235} \mathrm{U}$, and ${ }^{232} \mathrm{Th}$. As discussed in detail in Chapter 3, Samail peridotites have low $\mathrm{U}$ and Th concentrations. High-precision isotope dilution $\mathrm{U}$ and $\mathrm{Th}$ measurements indicate 
that altered, partially-serpentinized Samail peridotites have $U=0.0324 \pm 0.0143 \mathrm{ppm}$ $(1 \sigma, \mathrm{n}=6)$ and $\mathrm{Th}=0.0283 \pm 0.0317 \mathrm{ppm}(1 \sigma, \mathrm{n}=6)$. Less-altered Samail peridotites have lower concentrations with $U=0.0001 \pm 0.0001 \mathrm{ppm}(1 \sigma, \mathrm{n}=3)$ and $\mathrm{Th}=0.0054 \pm 0.0043$ ppm $(1 \sigma, \mathrm{n}=3)$. The ultramafic standards PCC-1 (peridotite, $U=0.0053 \mathrm{ppm}$ and $\mathrm{Th}=0.0049 \mathrm{ppm}$ ) and DTS-1 (dunite, $\mathrm{U}=0.0049 \mathrm{ppm}, \mathrm{Th}=0.0237 \mathrm{ppm}$ ) have $\mathrm{U}$ and Th concentrations in between those of the less-altered and more-altered Samail peridotites. As shown in Table 3, the amount of ${ }^{4} \mathrm{He}$ that could be produced from decay of $\mathrm{U}$ and $\mathrm{Th}$ over 95 million years exceeds the total ${ }^{4} \mathrm{He}$ content measured in most of the peridotite samples. This implies that: (1.) the Samail peridotites had lower $U$ and Th concentrations in the past and/or (2.) ${ }^{4} \mathrm{He}$ has been lost from the Samail peridotites during the past 95 million years.

The $\mathrm{U}$ and Th concentrations of Samail peridotites likely increase with time. Chapter 3 demonstrated that ongoing, low-temperature alteration of Samail peridotites adds $U$ (and possibly Th, although the mechanism for that is unclear). Furthermore, Chapter 3 demonstrated that there is disequilibria in the ${ }^{238} \mathrm{U}$ decay chain, which indicates that $U$ addition has occurred fairly recently within the past 350,000 years. The $U$ and $T h$ measurements in the less-altered Samail peridotites approach detection limits even for the high-precision isotope dilution technique, so it is possible that pristine mantle peridotite has even lower $U$ and Th concentrations. Significant loss of ${ }^{4} \mathrm{He}$ from peridotite is unlikely for pristine olivine and pyroxene due to extremely slow diffusion rates for helium in these minerals (e.g. Trull et al., 1991; Trull and Kurz, 1993). However, 
diffusion rates for ${ }^{4} \mathrm{He}$ in alteration phases such as serpentine are poorly constrained and could possibly be faster.

\section{4: Exposure Ages from Cosmogenic ${ }^{3} \mathrm{He}$ :}

Cosmogenic ${ }^{3} \mathrm{He}$ is generally determined using the following equations (e.g. Kurz et al., 1986b):

$$
{ }^{3} \mathrm{He}_{\mathrm{c}}={ }^{3} \mathrm{He}_{\mathrm{t}}-{ }^{3} \mathrm{He}_{\mathrm{i}}
$$

and

$$
{ }^{3} \mathrm{He}_{\mathrm{i}}={ }^{4} \mathrm{He}_{\mathrm{t}} \times\left({ }^{3} \mathrm{He} /{ }^{4} \mathrm{He}\right)_{\mathrm{i}}
$$

where the subscripts $\mathrm{c}, \mathrm{t}$, and $\mathrm{i}$ refer to the cosmogenic, total, and inherited helium, respectively. The inherited helium refers to any helium that is not cosmogenic and may originate from magmatic, atmospheric, and radiogenic sources. In the case of young basaltic phenocrysts, most of the magmatic helium is held within melt inclusions. Since crushing selectively releases magmatic helium from the melt inclusions (e.g. Kurz et al., 1986a, 1986b), it is possible to correct for inherited magmatic helium using the helium isotopic composition of the crushing measurement. This simple strategy above corrects for magmatic helium but does not account for radiogenic or atmospheric helium, and it is unclear if this procedure is valid for older basaltic flows or for altered ultramafic rocks. There are very few previous studies of peridotites, but the two existing studies with crush and melt data (Kurz et al., 2009; Recanati et al., submitted) indicate that most of the helium resides within the solid matrix rather than within melt or fluid inclusions.

Anticipating the presence of radiogenic helium in the Samail peridotites, we attempted to use a field strategy to evaluate the inherited components by collecting 
shielded samples, which should contain no cosmogenic helium, at the same outcrops where unshielded samples are collected. This approach has the limitations that appropriate shielded samples may not be accessible, there may be small-scale spatial variability in helium contents, and the shielded samples may differ in mineralogy or degree of alteration from the unshielded samples. For the Samail peridotites, the shielded samples had similar mineralogy to the associated unshielded samples but were often significantly less altered. If helium has been lost from the more-altered shielded samples, then this approach may lead to an overcorrection for inherited ${ }^{3} \mathrm{He}$.

Exposure ages (Table 4) were calculated for the six unshielded samples which have elevated cosmogenic ${ }^{3} \mathrm{He}$ in the melt fraction relative to the ${ }^{3} \mathrm{He}$ in the melt fraction of shielded samples from the same location. We calculated three sets of exposure ages using the following assumptions:

1. All ${ }^{3} \mathrm{He}$ in the melt fraction of the unshielded samples is cosmogenic. This assumption provides an upper limit for the exposure ages.

2. The cosmogenic ${ }^{3} \mathrm{He}$ is equal to the ${ }^{3} \mathrm{He}$ in the melt fraction of the unshielded sample minus the average of ${ }^{3} \mathrm{He}$ measured in the melt fractions of shielded samples from the same field location.

3. The $\left({ }^{3} \mathrm{He} /{ }^{4} \mathrm{He}\right)$ value of the crush fraction represents the inherited component, and therefore the inherited ${ }^{3} \mathrm{He}$ can be accounted for using Equations 1 and 2 .

Once cosmogenic ${ }^{3} \mathrm{He}$ was determined using these three assumptions, exposure ages were calculated by dividing by the ${ }^{3} \mathrm{He}$ production rate. The production rates were calculated using the scaling factors of Lal (1991) and the high-latitude, sea-level 
production rate of 120 atoms/g/yr from Goehring et al. (2010). Site production rates ranged from 136 to 149 atoms/g/yr. The exposure age ranges calculated using different assumptions are quite consistent $(\sim 11,000$ to $\sim 92,000$ yrs for assumption $\# 1$, from $\sim 3,000$ to $\sim 89,000$ yrs for assumption $\# 2$, and from $\sim 4,000$ to $\sim 91,000$ yrs for assumption \#3), indicating that the choice of assumption does not have a large effect on the determined exposure ages. An important consideration is that these ages represent minimum exposure ages because the calculation assumes that the samples have not been affected by erosion (see Figure 14) or shielding.

\section{5: Erosion Rates from Cosmogenic ${ }^{3} \mathrm{He}$ :}

Erosion rates were calculated using the following equation (e.g. Kurz et al., 1986b; Lal, 1991):

$$
N(t)=[P /(\epsilon / L)] x[1-\exp [-t(\epsilon / L)]] \quad(\text { Equation 3) }
$$

where $\mathrm{N}$ is the number of cosmogenic ${ }^{3} \mathrm{He}$ atoms in atoms $/ \mathrm{g}, \epsilon$ is the erosion rate in $\mathrm{g} / \mathrm{cm}^{2} / \mathrm{yr}, \mathrm{P}$ is the ${ }^{3} \mathrm{He}$ production rate in $\mathrm{cm} / \mathrm{g} / \mathrm{yr}$, and $\mathrm{L}$ is the apparent vertical attenuation length in $\mathrm{g} / \mathrm{cm}^{2}$. Dividing $\epsilon$ by the density $\rho$ of the rock gives the erosion rate in $\mathrm{cm} / \mathrm{yr}$. In the erosion rate calculations, we assumed that $\rho=3.3 \mathrm{~cm}^{3} / \mathrm{g}$ and $\mathrm{L}=160$ $\mathrm{g} / \mathrm{cm}^{2}$. This equation produces maximum erosion rates and assumes that the erosion rate has been constant over time. An example of how this equation is used to calculate maximum erosion rates (and also minimum exposure ages) is illustrated in Figure 14.

For the Samail peridotites analyzed in this study, maximum erosion rates were calculated using the cosmgoenic ${ }^{3} \mathrm{He}$ values obtained from the three assumptions described in Section 5.3. The erosion rates (Table 4) range from $\sim 5$ to $\sim 50 \mathrm{~m} / \mathrm{Myr}$ for 
assumption $\# 1$, from $\sim 6$ to $\sim 180 \mathrm{~m} / \mathrm{Myr}$ for assumption $\# 2$, and from $\sim 5$ to $122 \mathrm{~m} / \mathrm{Myr}$ for assumption \#3.

\subsection{Implications for Erosion of the Peridotite Layer of the Samail Ophiolite:}

\subsubsection{Ultramafic Erosion Rates:}

With the exception of the recent study by Recanati et al. (submitted), no prior studies have used cosmogenic isotopes to determine ultramafic bedrock exposure ages, and there are very few estimates for erosion rates of ultramafic bedrock. A field relation study employing xenoliths found in basaltic eruptions estimated erosion rates of 10 to 15 m/Myr for kimberlites in central South Africa (Hanson et al., 2009). A geochemical mass balance study of ultramafic schists in Amazonia, French Guiana, estimated that the saprotilization rate of the ultramafic bedrock is $\sim 7.5 \mathrm{~m} / \mathrm{Myr}$ (Freyssinet and Farah, 2000). These are within the range of the maximum erosion rates determined for Samail peridotite bedrock. A few studies (e.g. Moretti and Turcotte, 1985; Beauvais et al., 2007) have also investigated erosion of ultramafic rocks in New Caledonia but did not quantify the erosion rates specifically. However, these studies are probably not the best comparisons since they were carried out on different rock compositions and/or in more humid climates than Oman.

Since climate is a major factor influencing rock erosion rates (e.g. Portenga and Bierman, 2011), studies in arid environments may provide a better comparison for the Samail peridotite bedrock erosion rates. $\mathrm{A}{ }^{10} \mathrm{Be}$ and ${ }^{26} \mathrm{Al}$ cosmogenic study (Clapp et al., 2000) in Nahal Yael - a hyperarid drainage basin in Israel in granite schists and amphibolites - may provide a reasonable comparison since the climate of Nahal Yael is 
similar to that of northern Oman. Maximum erosion rates for Nahal Yael are $\sim 30 \mathrm{~m} / \mathrm{Myr}$ (Clapp et al., 2000). This is very similar to the average maximum erosion rate (assumptions \#1 and \#2) of $\sim 40 \mathrm{~m} / \mathrm{Myr}$ determined for Samail peridotites.

Prior to this study, the only estimate for erosion rates in the Samail Ophiolite came from an apatite fission-track thermochronology study (Poupeau et al., 1998) in high-pressure metamorphic rocks of continental origin underneath the Samail Ophiolite rocks. This study indicated that Paleocene and Pliocene-Quaternary denudation rates are $\sim 300 \mathrm{~m} / \mathrm{Myr}$ for northern Oman. Poupeau et al. (1998) attributed this denudation to isostatically-assisted erosion, so this number represents an upper estimate for erosion rates in Oman. The average erosion rate of $\sim 40 \mathrm{~m} / \mathrm{Myr}$ determined in this study also represents an upper estimate, but is significantly lower than the rate constraint from Poupeau et al. (1998).

\subsubsection{Implications for Natural $\mathrm{CO}_{2}$ Sequestration in the Samail Ophiolite:}

A major goal of Chapters 2 and 3 was improving estimates of the natural $\mathrm{CO}_{2}$ uptake rate in secondary carbonate minerals via ongoing, low-temperature alteration of the peridotite layer of the Samail Ophiolite. In those chapters, it was estimated that $\mathrm{Mg}$ rich carbonate veins that form in peridotite uptake approximately $10^{7} \mathrm{~kg} \mathrm{CO}_{2} / \mathrm{yr}$. As the peridotite bedrock erodes, the Mg-rich carbonate veins will also erode and, in the process, transfer the $\mathrm{CO}_{2}$ they contain to other reservoirs, such as sedimentary deposits and the hydrosphere. The maximum erosion rates determined in this chapter permit the determination of an upper limit estimate of the rates of $\mathrm{CO}_{2}$ loss through erosion of peridotite bedrock. Using the erosion rates of $\sim 5-180 \mathrm{~m} / \mathrm{Myr}$ (average: $\sim 40 \mathrm{~m} / \mathrm{Myr}$ ) and 
assuming that the Samail Ophiolite is $\sim 40 \mathrm{~km}$ wide, $\sim 350 \mathrm{~km}$ long, and contains $\sim 30 \%$ mantle peridotite which contains $\sim 1 \% \mathrm{Mg}$-rich carbonate veins, then $\sim 10^{5}-10^{6} \mathrm{~kg} \mathrm{CO} / \mathrm{yr}$ will be lost through erosion of peridotite bedrock. This upper estimate is an order of magnitude lower than the estimate of $\mathrm{CO}_{2}$ uptake through low-temperature alteration of peridotite.

\section{Summary:}

Helium concentrations and isotopes were measured in olivine, pyroxene, and whole rock fractions from partially-serpentinized peridotite bedrock in the Samail Ophiolite, Oman. Helium relations are complex, and the samples contain mixtures of mantle, radiogenic, atmospheric, and radiogenic helium. One sample (OM09-4P-MS) has unambiguous cosmogenic ${ }^{3} \mathrm{He}$ with $\mathrm{R} / \mathrm{R}_{\mathrm{A}}=218$ while other samples likely contain cosmogenic ${ }^{3} \mathrm{He}$ due to: 1.) elevated ${ }^{3} \mathrm{He} /{ }^{4} \mathrm{He}$ ratios relative to typical MORB and ultramafic xenoliths values and/or 2.) elevated ${ }^{3} \mathrm{He}$ concentrations in unshielded samples relative to shielded samples from the sample field locations. Minimum exposure ages and minimum erosion rates were determined for the peridotite samples with elevated ${ }^{3} \mathrm{He}$ concentrations. The minimum exposure ages range from $\sim 3,000$ to $91,000 \mathrm{yrs}$, and the maximum erosion rates range from $\sim 5$ to $180 \mathrm{~m} / \mathrm{Myr}$. The maximum erosion rates suggest that at most $\sim 10^{5}-10^{6} \mathrm{~kg} \mathrm{CO}_{2} / \mathrm{yr}$ is lost through erosion of peridotite bedrock in the Samail Ophiolite.

\section{Acknowledgments:}

This research was done in collaboration with Mark Kurz and my supervisors, Kenneth Sims, Susan Humphris, and Peter Kelemen. Steve Richardson and Henry Dick 
are thanked for assistance with thin section preparations and descriptions. Margaret Sulanowska is thanked for assistance with X-ray diffraction analysis. Ali Al-Rajhi, Ministry of Commerce and Industry, Sultanate of Oman, and Sobhi Nasir, Sultan Qaboos University, are thanked for logistical support during field seasons. I also thank Jürg Matter, Everett Shock, Lisa Streit, Amelia Paukert, Ernest Gauntlett, and others for assistance with fieldwork in Oman and for scientific discussions. This research was funded by National Science Foundation grant EAR-1049281, the Deep Ocean Exploration Institute at Woods Hole Oceanographic Institution, the Mellon Foundation, the Academic Programs Office at Woods Hole Oceanographic Institution, and a Research Initiative in Science and Engineering Grant from Columbia University to Peter Kelemen and Jürg Matter and also Kelemen's Charles Francis Adams Chair at Columbia University. 
Tables:

Table 1: Sample Descriptions and Mineralogy

\begin{tabular}{|c|c|c|c|c|c|c|c|}
\hline $\begin{array}{l}\text { Sample } \\
\text { Name: }\end{array}$ & Location: & Shielded?: & $\begin{array}{l}\text { UTM- } \\
\text { Easting: }\end{array}$ & $\begin{array}{l}\text { UTM- } \\
\text { Northing: }\end{array}$ & Elevation (m): & $\begin{array}{l}\text { Major Minerals } \\
\text { (XRD): }\end{array}$ & $\begin{array}{l}\text { Trace Minerals } \\
\text { (XRD): }\end{array}$ \\
\hline $\begin{array}{l}\text { OM09-2P- } \\
\text { MS }\end{array}$ & $\begin{array}{c}\text { Faleej } \\
\text { Travertine }\end{array}$ & No & 0600421 & 2525962 & 540 & $\begin{array}{c}\text { chrysotile, forsterite, } \\
\text { enstatite }\end{array}$ & spinel \\
\hline $\begin{array}{c}\text { OM10-2P- } \\
\text { MG }\end{array}$ & $\begin{array}{c}\text { Fanja } \\
\text { Roadcut }\end{array}$ & No & 0609451 & 2597416 & 281 & chrysotile, forsterite & magnetite, hydrotalcite \\
\hline $\begin{array}{l}\text { OM10-3P- } \\
\text { MG }\end{array}$ & $\begin{array}{c}\text { Fanja } \\
\text { Roadcut }\end{array}$ & No & 0609451 & 2597416 & 281 & chrysotile, forsterite & $\begin{array}{l}\text { hydrotalcite, diopside } \\
\text { (very small amount) }\end{array}$ \\
\hline $\begin{array}{l}\text { OM10-4P- } \\
\text { MG }\end{array}$ & $\begin{array}{c}\text { Fanja } \\
\text { Roadcut }\end{array}$ & Yes & 0609372 & 2597494 & 248 & $\begin{array}{c}\text { chrysotile, forsterite, } \\
\text { hydrotalcite }\end{array}$ & magnetite \\
\hline $\begin{array}{l}\text { OM09-4P- } \\
\text { MS }\end{array}$ & $\begin{array}{l}\text { Qafeefah } \\
\text { Travertine }\end{array}$ & No & 0646145 & 2533666 & 660 & lizardite & $\begin{array}{l}\text { enstatite, diopside, } \\
\text { spinel }\end{array}$ \\
\hline $\begin{array}{l}\text { OM10-9P- } \\
\text { MG }\end{array}$ & $\begin{array}{l}\text { Qafeefah } \\
\text { Travertine }\end{array}$ & No & 0646171 & 2533693 & 670 & lizardite & $\begin{array}{l}\text { forsterite, enstatite, } \\
\text { hydrotalcite, spinel }\end{array}$ \\
\hline $\begin{array}{c}\text { OM10-11P- } \\
\text { MG }\end{array}$ & $\begin{array}{l}\text { Qafeefah } \\
\text { Travertine }\end{array}$ & No & 0646106 & 2533761 & 710 & chrysotile, forsterite & hydrotalcite, spinel \\
\hline $\begin{array}{c}\text { OM10-10P- } \\
\text { MG }\end{array}$ & $\begin{array}{l}\text { Qafeefah } \\
\text { Roadcut }\end{array}$ & Yes & 0646216 & 2536749 & 725 & chrysotile, forsterite & $\begin{array}{c}\text { talc, spinel, magnetite, } \\
\text { diopside (very small } \\
\text { amount) }\end{array}$ \\
\hline $\begin{array}{c}\text { OM10-13P- } \\
\text { MG }\end{array}$ & $\begin{array}{c}\text { Misht } \\
\text { Travertine }\end{array}$ & Yes & 0487305 & 2576134 & 630 & chrysotile, calcite & forsterite, spinel \\
\hline $\begin{array}{c}\text { OM10-14P- } \\
\text { MG }\end{array}$ & $\begin{array}{c}\text { Misht } \\
\text { Travertine }\end{array}$ & No & 0487233 & 2576099 & 660 & chrysotile, forsterite & $\begin{array}{l}\text { enstatite, talc, } \\
\text { hydrotalcite }\end{array}$ \\
\hline $\begin{array}{c}\text { OM10-17P- } \\
\text { MG }\end{array}$ & $\begin{array}{c}\text { Misht } \\
\text { Travertine }\end{array}$ & No & 0489565 & 2575412 & 678 & chrysotile, forsterite & enstatite, spinel \\
\hline $\begin{array}{c}\text { OM10-19P- } \\
\text { MG }\end{array}$ & $\begin{array}{c}\text { Wadi } \\
\text { Sudari } \\
\text { Travertine }\end{array}$ & No & 0443154 & 2650213 & 660 & lizardite & spinel \\
\hline $\begin{array}{c}\text { OM10-20P- } \\
\text { MG }\end{array}$ & $\begin{array}{c}\text { Wadi } \\
\text { Sudari } \\
\text { Travertine }\end{array}$ & Yes & 0443046 & 2650317 & 676 & $\begin{array}{c}\text { chrysotile, forsterite, } \\
\text { enstatite }\end{array}$ & spinel, talc, hydrotalcite \\
\hline
\end{tabular}




\begin{tabular}{|c|c|c|c|c|c|c|c|}
\hline $\begin{array}{c}\text { Sample } \\
\text { Name: }\end{array}$ & Location: & Shielded?: & $\begin{array}{c}\text { UTM- } \\
\text { Easting: }\end{array}$ & $\begin{array}{c}\text { UTM- } \\
\text { Northing: }\end{array}$ & Elevation (m): & $\begin{array}{c}\text { Major Minerals } \\
\text { (XRD): }\end{array}$ & $\begin{array}{c}\text { Trace Minerals } \\
\text { (XRD): }\end{array}$ \\
\hline $\begin{array}{c}\text { OM10-22P- } \\
\text { MG }\end{array}$ & $\begin{array}{c}\text { Wadi } \\
\text { Sudari } \\
\text { Campsite }\end{array}$ & No & 0445924 & 2647613 & 587 & $\begin{array}{c}\text { chrysotile, forsterite, } \\
\text { enstatite }\end{array}$ & diopside, spinel \\
\hline
\end{tabular}


Table 2: He Concentrations and Isotopic Measurements

\begin{tabular}{|c|c|c|c|c|c|c|c|c|c|c|}
\hline $\begin{array}{l}\text { Sample } \\
\text { Name: }\end{array}$ & $\begin{array}{c}\text { Wt. } \\
\text { Crush } \\
\text { (g): }\end{array}$ & $\begin{array}{l}{ }^{3} \mathrm{He} /{ }^{4} \mathrm{He} \\
\text { Crush } \\
\left(\mathbf{R} / \mathbf{R}_{\mathbf{A}}\right):\end{array}$ & $\begin{array}{c}{ }^{4} \text { He Crush } \\
\text { (cc/g): }\end{array}$ & $\begin{array}{l}{ }^{3} \mathrm{He} \\
\text { Crush } \\
\text { (ce/g): }\end{array}$ & $\begin{array}{l}\text { Wt. } \\
\text { Melt } \\
\text { (g): }\end{array}$ & $\begin{array}{c}{ }^{3} \mathbf{H e} /{ }^{4} \mathrm{He} \\
\text { Melt } \\
\left(\mathbf{R} / \mathbf{R}_{\mathbf{A}}\right):\end{array}$ & $\begin{array}{c}{ }^{4} \text { He Melt } \\
\text { (cc/g): }\end{array}$ & $\begin{array}{c}{ }^{3} \mathrm{He} \text { Melt } \\
\text { (cc/g): }\end{array}$ & $\begin{array}{c}\text { Total } \\
{ }^{4} \mathrm{He} \\
(\mathrm{cc} / \mathrm{g}):\end{array}$ & $\begin{array}{c}\text { Fraction } \\
\text { Crush: }\end{array}$ \\
\hline $\begin{array}{l}\text { OM09-2P- } \\
\text { MG-olivine }\end{array}$ & 0.28332 & $1.53 \pm 0.09$ & 2.619E-09 & $5.546 \mathrm{E}-15$ & 0.26964 & $4.79 \pm 0.07$ & $2.497 \mathrm{E}-08$ & $1.655 \mathrm{E}-13$ & $\begin{array}{c}2.759 \mathrm{E}- \\
08\end{array}$ & 0.09 \\
\hline $\begin{array}{l}\text { OM09-2P- } \\
\text { MG-pyroxene }\end{array}$ & 0.22167 & $1.34 \pm 0.08$ & $3.387 \mathrm{E}-09$ & $6.281 \mathrm{E}-15$ & 0.21276 & $4.85 \pm 0.08$ & $2.713 \mathrm{E}-08$ & $1.821 \mathrm{E}-13$ & $\begin{array}{c}3.052 \mathrm{E}- \\
08\end{array}$ & 0.11 \\
\hline $\begin{array}{l}\text { OM09-2P- } \\
\text { MG-whole } \\
\text { rock }\end{array}$ & 0.32227 & $1.95 \pm 0.25$ & $4.946 \mathrm{E}-10$ & $1.335 \mathrm{E}-15$ & 0.30801 & $5.68 \pm 0.10$ & $7.874 \mathrm{E}-09$ & $6.190 \mathrm{E}-14$ & $\begin{array}{c}8.369 \mathrm{E}- \\
09\end{array}$ & 0.06 \\
\hline $\begin{array}{l}\text { OM10-2P- } \\
\text { MG-whole } \\
\text { rock }\end{array}$ & 0.26212 & $7.83 \pm 0.11$ & $7.987 \mathrm{E}-08$ & $8.655 \mathrm{E}-13$ & 0.25212 & $7.74 \pm 0.11$ & $8.459 \mathrm{E}-07$ & $9.061 \mathrm{E}-12$ & $\begin{array}{c}9.257 \mathrm{E}- \\
07\end{array}$ & 0.09 \\
\hline $\begin{array}{l}\text { OM10-3P- } \\
\text { MG-altered } \\
\text { olivine }\end{array}$ & 0.24846 & $5.91 \pm 0.12$ & $6.274 \mathrm{E}-09$ & $5.132 \mathrm{E}-14$ & 0.24122 & $7.79 \pm 0.10$ & $6.902 \mathrm{E}-08$ & $7.441 \mathrm{E}-13$ & $\begin{array}{c}7.529 \mathrm{E}- \\
08 \\
\end{array}$ & 0.08 \\
\hline $\begin{array}{c}\text { OM10-4P- } \\
\text { MG-altered } \\
\text { olivine? }\end{array}$ & 0.25831 & $8.41 \pm 0.12$ & $1.941 \mathrm{E}-07$ & $2.259 \mathrm{E}-12$ & 0.24853 & $8.15 \pm 0.13$ & $1.320 \mathrm{E}-06$ & $1.489 \mathrm{E}-11$ & $\begin{array}{c}1.514 \mathrm{E}- \\
06\end{array}$ & 0.13 \\
\hline $\begin{array}{l}\text { OM10-4P- } \\
\text { MG-whole } \\
\text { rock }\end{array}$ & 0.16899 & $7.93 \pm 0.12$ & $1.115 \mathrm{E}-07$ & $1.224 \mathrm{E}-12$ & 0.16117 & $7.30 \pm 0.10$ & $1.059 \mathrm{E}-06$ & $1.070 \mathrm{E}-11$ & $\begin{array}{c}1.170 \mathrm{E}- \\
06\end{array}$ & 0.10 \\
\hline $\begin{array}{l}\text { OM09-4P-MS- } \\
\text { pyroxene }\end{array}$ & 0.27793 & $0.97 \pm 0.76$ & $8.229 \mathrm{E}-11$ & $1.105 \mathrm{E}-16$ & 0.26565 & $\begin{array}{c}218.98 \pm \\
2.83 \\
\end{array}$ & $1.618 \mathrm{E}-09$ & $4.902 \mathrm{E}-13$ & $\begin{array}{c}1.700 \mathrm{E}- \\
09\end{array}$ & 0.05 \\
\hline $\begin{array}{l}\text { OM10-9P- } \\
\text { MG-whole } \\
\text { rock }\end{array}$ & 0.29864 & $1.45 \pm 0.10$ & $1.066 \mathrm{E}-09$ & $2.139 \mathrm{E}-15$ & 0.29006 & $\begin{array}{c}10.60 \pm \\
0.31 \\
\end{array}$ & $3.787 \mathrm{E}-09$ & $5.554 \mathrm{E}-14$ & $\begin{array}{c}4.853 \mathrm{E}- \\
09\end{array}$ & 0.22 \\
\hline $\begin{array}{c}\text { OM10-11P- } \\
\text { MG-altered } \\
\text { olivine }\end{array}$ & 0.25955 & $3.03 \pm 0.16$ & $1.033 \mathrm{E}-09$ & 4.332E-15 & 0.24746 & $4.61 \pm 0.13$ & $1.272 \mathrm{E}-08$ & $8.107 \mathrm{E}-14$ & $\begin{array}{c}1.375 \mathrm{E}- \\
08 \\
\end{array}$ & 0.08 \\
\hline $\begin{array}{l}\text { OM10-11P- } \\
\text { MG-whole } \\
\text { rock }\end{array}$ & 0.29486 & $3.30 \pm 0.15$ & $8.518 \mathrm{E}-10$ & $3.890 \mathrm{E}-15$ & 0.28301 & $5.08 \pm 0.09$ & 8.852E-09 & $6.224 \mathrm{E}-14$ & $\begin{array}{c}9.703 \mathrm{E}- \\
09 \\
\end{array}$ & 0.09 \\
\hline
\end{tabular}




\begin{tabular}{|c|c|c|c|c|c|c|c|c|c|c|}
\hline $\begin{array}{l}\text { Sample } \\
\text { Name: }\end{array}$ & $\begin{array}{c}\text { Wt. } \\
\text { Crush } \\
\text { (g): }\end{array}$ & $\begin{array}{l}{ }^{3} \mathbf{H e} /{ }^{4} \mathbf{H e} \\
\text { Crush } \\
\left(\mathbf{R} / \mathbf{R}_{\mathrm{A}}\right):\end{array}$ & $\begin{array}{c}{ }^{4} \mathrm{He} \text { Crush } \\
\text { (cc/g): }\end{array}$ & $\begin{array}{c}{ }^{3} \mathrm{He} \\
\text { Crush } \\
\text { (cc/g): }\end{array}$ & $\begin{array}{l}\text { Wt. } \\
\text { Melt } \\
\text { (g): }\end{array}$ & $\begin{array}{c}{ }^{3} \mathbf{H e} /{ }^{4} \mathbf{H e} \\
\text { Melt } \\
\left(\mathbf{R} / \mathbf{R}_{\mathbf{A}}\right):\end{array}$ & $\begin{array}{c}{ }^{4} \text { He Melt } \\
\text { (cc/g): }\end{array}$ & $\begin{array}{c}{ }^{3} \text { He Melt } \\
\text { (ce/g): }\end{array}$ & $\begin{array}{c}\text { Total } \\
{ }^{4} \mathrm{He} \\
\text { (cc/g): }\end{array}$ & $\begin{array}{l}\text { Fraction } \\
\text { Crush: }\end{array}$ \\
\hline $\begin{array}{l}\text { OM10-10P- } \\
\text { MG-altered } \\
\text { olivine }\end{array}$ & 0.25909 & $3.68 \pm 0.33$ & $1.033 \mathrm{E}-09$ & $5.261 \mathrm{E}-15$ & 0.24746 & $5.69 \pm 0.16$ & $2.314 \mathrm{E}-09$ & $1.822 \mathrm{E}-14$ & $\begin{array}{c}3.347 \mathrm{E}- \\
09\end{array}$ & 0.31 \\
\hline $\begin{array}{l}\text { OM10-10P- } \\
\text { MG-whole } \\
\text { rock }\end{array}$ & 0.23749 & $2.96 \pm 0.26$ & $8.518 \mathrm{E}-10$ & $3.489 \mathrm{E}-15$ & 0.28301 & $3.81 \pm 0.13$ & $2.662 \mathrm{E}-09$ & $1.403 \mathrm{E}-14$ & $\begin{array}{c}3.514 \mathrm{E}- \\
09\end{array}$ & 0.24 \\
\hline $\begin{array}{l}\text { OM10-13P- } \\
\text { MG-whole } \\
\text { rock }\end{array}$ & 0.27359 & $0.42 \pm 0.10$ & $7.589 \mathrm{E}-10$ & $4.411 \mathrm{E}-16$ & 0.26179 & $0.91 \pm 0.03$ & $5.011 \mathrm{E}-09$ & $6.311 \mathrm{E}-15$ & $\begin{array}{c}5.770 \mathrm{E}- \\
09\end{array}$ & 0.13 \\
\hline $\begin{array}{c}\text { OM10-14P- } \\
\text { MG-pyroxene }\end{array}$ & 0.22681 & $0.76 \pm 0.21$ & $4.247 \mathrm{E}-10$ & 4.467E-16 & 0.22013 & $\begin{array}{c}29.95 \pm \\
0.46\end{array}$ & $2.996 \mathrm{E}-09$ & $1.242 \mathrm{E}-13$ & $\begin{array}{c}3.420 \mathrm{E}- \\
09\end{array}$ & 0.12 \\
\hline $\begin{array}{l}\text { OM10-14P- } \\
\text { MG-whole } \\
\text { rock }\end{array}$ & 0.29748 & $0.88 \pm 0.15$ & $5.906 \mathrm{E}-10$ & $7.193 \mathrm{E}-16$ & 0.28413 & $\begin{array}{c}19.02 \pm \\
0.35\end{array}$ & $2.883 \mathrm{E}-09$ & $7.588 \mathrm{E}-14$ & $\begin{array}{c}3.473 \mathrm{E}- \\
09\end{array}$ & 0.17 \\
\hline $\begin{array}{l}\text { OM10-17P- } \\
\text { MG-pyroxene }\end{array}$ & 0.27720 & $0.22 \pm 0.03$ & $2.903 \mathrm{E}-09$ & $8.839 \mathrm{E}-16$ & 0.26606 & $6.35 \pm 0.18$ & $1.979 \mathrm{E}-08$ & $1.739 \mathrm{E}-13$ & $\begin{array}{c}2.269 \mathrm{E}- \\
08\end{array}$ & 0.13 \\
\hline $\begin{array}{l}\text { OM10-19P- } \\
\text { MG-whole } \\
\text { rock }\end{array}$ & 0.28658 & $0.21 \pm 0.15$ & $3.52048 \mathrm{E}-10$ & $1.023 \mathrm{E}-16$ & 0.27342 & $1.35 \pm 0.06$ & $\begin{array}{c}3.8324 \mathrm{E}- \\
09\end{array}$ & $7.160 \mathrm{E}-15$ & $\begin{array}{c}4.184 \mathrm{E}- \\
09\end{array}$ & 0.08 \\
\hline $\begin{array}{l}\text { OM10-20P- } \\
\text { MG-olivine }\end{array}$ & 0.26973 & $6.33 \pm 0.26$ & $6.58881 \mathrm{E}-10$ & $5.772 \mathrm{E}-15$ & 0.25802 & $5.57 \pm 0.16$ & $\begin{array}{c}2.1658 \mathrm{E}- \\
09\end{array}$ & $1.670 \mathrm{E}-14$ & $\begin{array}{c}2.825 \mathrm{E}- \\
09\end{array}$ & 0.23 \\
\hline $\begin{array}{l}\text { OM10-20P- } \\
\text { MG-pyroxene }\end{array}$ & 0.26639 & $0.33 \pm 0.06$ & $1.48072 \mathrm{E}-09$ & $6.763 \mathrm{E}-16$ & 0.25871 & $0.61 \pm 0.02$ & $\begin{array}{c}2.8830 \mathrm{E}- \\
08\end{array}$ & $2.434 \mathrm{E}-14$ & $\begin{array}{c}3.031 \mathrm{E}- \\
08\end{array}$ & 0.05 \\
\hline $\begin{array}{l}\text { OM10-20P- } \\
\text { MG-whole } \\
\text { rock }\end{array}$ & 0.23978 & $0.28 \pm 0.01$ & $3.16354 \mathrm{E}-08$ & $1.226 \mathrm{E}-14$ & 0.23355 & $0.35 \pm 0.01$ & $\begin{array}{c}2.1780 \mathrm{E}- \\
07\end{array}$ & $1.055 \mathrm{E}-13$ & $\begin{array}{c}2.494 \mathrm{E}- \\
07\end{array}$ & 0.13 \\
\hline $\begin{array}{l}\text { OM10-22P- } \\
\text { MG-olivine }\end{array}$ & 0.23232 & $0.30 \pm 0.01$ & $2.9453 \mathrm{E}-08$ & $1.223 \mathrm{E}-14$ & 0.22457 & $1.22 \pm 0.01$ & $\begin{array}{c}1.3014 \mathrm{E}- \\
07\end{array}$ & $2.197 \mathrm{E}-13$ & $\begin{array}{c}1.596 \mathrm{E}- \\
07\end{array}$ & 0.18 \\
\hline $\begin{array}{l}\text { OM10-22P- } \\
\text { MG-pyroxene }\end{array}$ & 0.20564 & $0.26 \pm 0.01$ & $7.21577 \mathrm{E}-08$ & $2.597 \mathrm{E}-14$ & 0.19729 & $1.09 \pm 0.02$ & $\begin{array}{c}2.0070 \mathrm{E}- \\
07\end{array}$ & $3.028 \mathrm{E}-13$ & $\begin{array}{c}2.729 \mathrm{E}- \\
07\end{array}$ & 0.26 \\
\hline
\end{tabular}

${ }^{4} \mathrm{He}$ measurements have $\sim 1-2 \%$ errors. 
Table 3: Comparison of ${ }^{4} \mathrm{He}$ Production from $\mathrm{U}$ and $\mathrm{Th}$ in $95 \mathrm{Myr}$ and ${ }^{4} \mathrm{He}$ Concentration Measured (in \%)

\begin{tabular}{|c|c|c|c|}
\hline Sample Name: & ${ }^{4} \mathrm{He}$ Total (cc/g): & $\%{ }^{4}$ He from Radiogenic- Low Estimate: & $\%{ }^{4}$ He from Radiogenic- High Estimate: \\
\hline OM09-2P-MG-olivine & $2.76 \mathrm{E}-08$ & 103 & 1861 \\
\hline OM09-2P-MG-pyroxene & $3.05 \mathrm{E}-08$ & 93 & 1682 \\
\hline OM09-2P-MG-wholerock & $8.37 \mathrm{E}-09$ & 339 & 6135 \\
\hline OM10-2P-MG-whole rock & $9.26 \mathrm{E}-07$ & 3 & 55 \\
\hline OM10-3P-MG-altered olivine & $7.53 \mathrm{E}-08$ & 38 & 682 \\
\hline OM10-4P-MG-altered olivine & $1.51 \mathrm{E}-06$ & 2 & 34 \\
\hline OM10-4P-MG-whole rock & $1.17 \mathrm{E}-06$ & 2 & 44 \\
\hline OM09-4P-MS-pyroxene & $1.70 \mathrm{E}-09$ & 1671 & 30205 \\
\hline OM10-9P-MG-whole rock & $4.85 \mathrm{E}-09$ & 585 & 10580 \\
\hline OM10-11P-MG-altered olivine & $1.37 \mathrm{E}-08$ & 207 & 3734 \\
\hline OM10-11P-MG-whole rock & $9.70 \mathrm{E}-09$ & 293 & 5291 \\
\hline OM10-10P-MG-altered olivine & $3.35 \mathrm{E}-09$ & 848 & 15340 \\
\hline OM10-10P-MG-whole rock & $3.51 \mathrm{E}-09$ & 808 & 14611 \\
\hline OM10-13P-MG-whole rock & $5.77 \mathrm{E}-09$ & 492 & 8898 \\
\hline OM10-14P-MG-pyroxene & $3.42 \mathrm{E}-09$ & 830 & 15012 \\
\hline OM10-14P-MG-whole rock & $3.47 \mathrm{E}-09$ & 818 & 14783 \\
\hline OM10-17P-MG-pyroxene & $2.27 \mathrm{E}-08$ & 125 & 2263 \\
\hline OM10-19P-MG-wholerock & $4.18 \mathrm{E}-09$ & 679 & 12270 \\
\hline OM10-20P-MG-olivine & $2.82 \mathrm{E}-09$ & 1005 & 18177 \\
\hline OM10-20P-MG-pyroxene & $3.03 \mathrm{E}-08$ & 94 & 1694 \\
\hline OM10-20P-MG-whole rock & $2.49 \mathrm{E}-07$ & 11 & 206 \\
\hline OM10-22P-MG-olivine & $1.60 \mathrm{E}-07$ & 18 & 322 \\
\hline OM10-22P-MG-pyroxene & $2.73 \mathrm{E}-07$ & 10 & 188 \\
\hline
\end{tabular}

Estimates of radiogenic ${ }^{4} \mathrm{He}$ production from decay of $\mathrm{U}$ and $\mathrm{Th}$ are discussed in the text. The low estimate uses $\mathrm{U}=0.0001$ $\mathrm{ppm}$ and $\mathrm{Th}=0.005 \mathrm{ppm}$ (average of less-altered Samail peridotites presented in Chapter 3), and the high estimate uses $U=$ $0.04 \mathrm{ppm}$ and $\mathrm{Th}=0.03 \mathrm{ppm}$ (average of altered Samail peridotites. Percentages less than 100 are indicated in bold while impossible percentages greater than $100 \%$ are indicated in italics. 
Table 4: Minimum Exposure Ages and Maximum Erosion Rates

\begin{tabular}{|c|c|c|c|c|c|c|c|c|c|}
\hline & Assumption \#1: & & & Assumption $\# 2$ : & & & Assumption \#3: & & \\
\hline $\begin{array}{l}\text { Sample } \\
\text { Name: }\end{array}$ & $\begin{array}{c}\text { Cosmogenic } \\
{ }^{3} \mathrm{He}(\text { atoms } / \mathrm{g}):\end{array}$ & $\begin{array}{l}\text { Exposure } \\
\text { age (yrs): }\end{array}$ & $\begin{array}{c}\text { Erosion } \\
\text { Rate } \\
(\mathrm{m} / \mathrm{Myr}):\end{array}$ & $\begin{array}{c}\text { Cosmogenic }{ }^{3} \mathrm{He} \\
\text { (atoms/g): }\end{array}$ & $\begin{array}{l}\text { Exposure } \\
\text { age (yrs): }\end{array}$ & $\begin{array}{c}\text { Erosion Rate } \\
(\mathrm{m} / \mathrm{Myr}):\end{array}$ & $\begin{array}{c}\text { Cosmogenic }{ }^{3} \mathrm{He} \\
\text { (atoms } / \mathrm{g}):\end{array}$ & $\begin{array}{l}\text { Exposure } \\
\text { age (yrs): }\end{array}$ & $\begin{array}{c}\text { Erosion } \\
\text { Rate } \\
\text { (m/Myr): }\end{array}$ \\
\hline $\begin{array}{c}\text { OM09-4P- } \\
\text { MS-pyroxene }\end{array}$ & $1.32 \mathrm{E}+07$ & 91524 & 5 & $1.27 \mathrm{E}+07$ & 88513 & 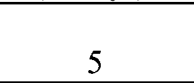 & $1.31 \mathrm{E}+07$ & 91117 & 5 \\
\hline $\begin{array}{l}\text { OM10-9P- } \\
\text { MG-whole } \\
\text { rock }\end{array}$ & $1.49 E+06$ & 10297 & 47 & $1.06 \mathrm{E}+06$ & 7307 & 66 & $1.29 \mathrm{E}+06$ & 8888 & 55 \\
\hline $\begin{array}{l}\text { OM10-11P- } \\
\text { MG-pyroxene }\end{array}$ & $2.18 \mathrm{E}+06$ & 14628 & 33 & $1.75 \mathrm{E}+06$ & 11718 & 41 & $7.48 \mathrm{E}+05$ & 5018 & 97 \\
\hline $\begin{array}{l}\text { OM10-11P- } \\
\text { MG-whole } \\
\text { rock }\end{array}$ & $1.67 \mathrm{E}+06$ & 11231 & 43 & $3.95 \mathrm{E}+05$ & 2651 & 183 & $5.88 \mathrm{E}+05$ & 3945 & 123 \\
\hline $\begin{array}{l}\text { OM10-14P- } \\
\text { MG-pyroxene }\end{array}$ & $3.34 \mathrm{E}+06$ & 23178 & 21 & $3.17 \mathrm{E}+06$ & 21998 & 22 & $3.25 E+06$ & 22590 & 21 \\
\hline $\begin{array}{l}\text { OM10-14P- } \\
\text { MG-whole } \\
\text { rock }\end{array}$ & $2.04 \mathrm{E}+06$ & 14162 & 34 & $1.87 \mathrm{E}+06$ & 12983 & 37 & $1.94 \mathrm{E}+06$ & 13506 & 36 \\
\hline $\begin{array}{l}\text { OM10-17P- } \\
\text { MG- whole } \\
\text { rock }\end{array}$ & $4.68 \mathrm{E}+06$ & 32043 & 15 & $4.51 \mathrm{E}+06$ & 30879 & 16 & $4.51 \mathrm{E}+06$ & 30906 & 16 \\
\hline $\begin{array}{l}\text { OM10-22P- } \\
\text { MG-olivine }\end{array}$ & $5.90 \mathrm{E}+06$ & 43359 & 11 & $4.57 E+06$ & 33621 & 14 & $4.47 \mathrm{E}+06$ & 32834 & 15 \\
\hline $\begin{array}{l}\text { OM10-22P- } \\
\text { MG-pyroxene }\end{array}$ & $8.15 E+06$ & 59928 & 8 & $6.83 E+06$ & 50190 & 10 & $6.18 \mathrm{E}+06$ & 45470 & 11 \\
\hline
\end{tabular}


Figures:

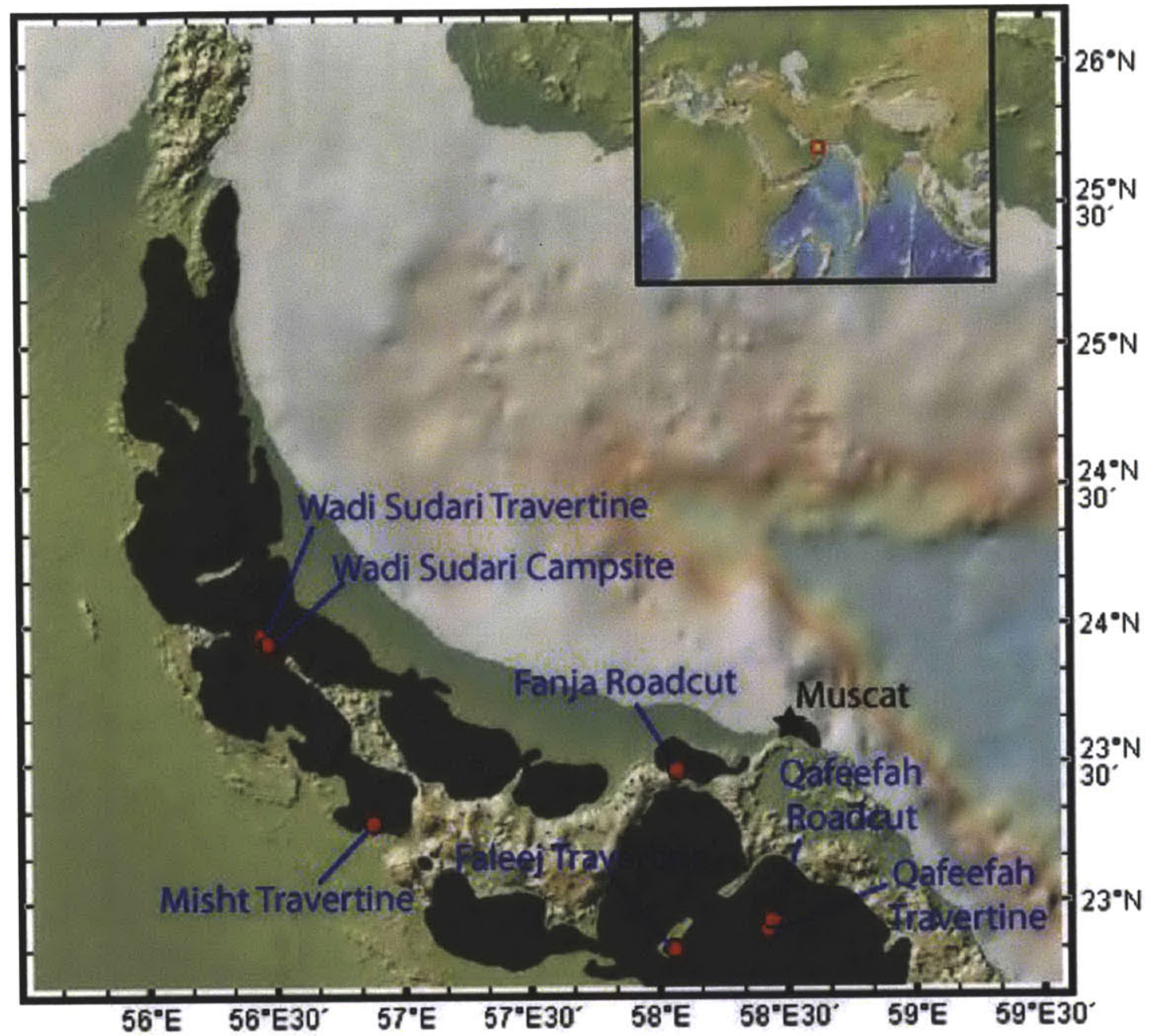

Figure 1: The seven field locations where peridotite samples were collected during 2009 and 2010 field seasons. The Samail Ophiolite is outlined in dark gray, and sampling locations are indicated by red circles. Base map from GeoMapApp. 


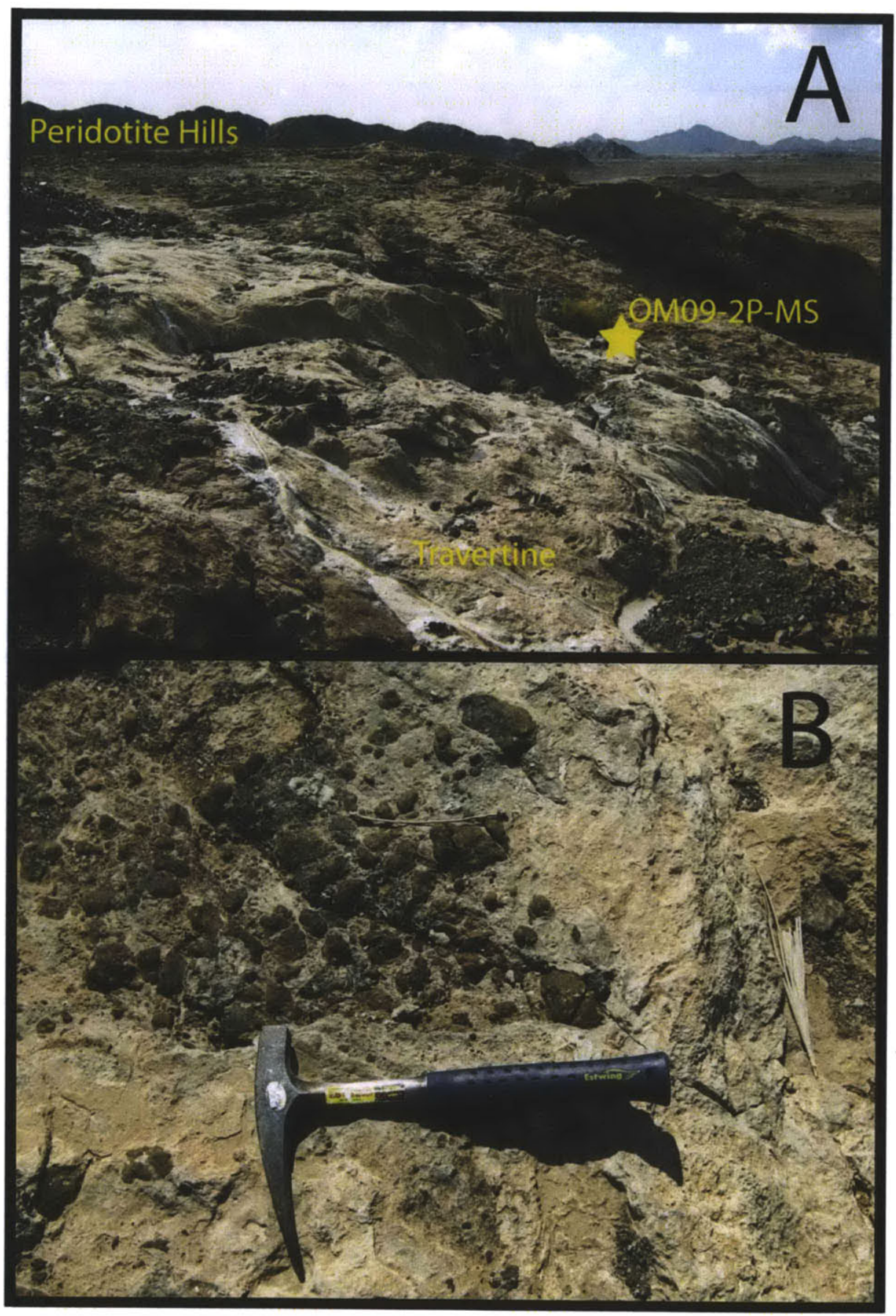

Figure 2: Faleej Travertine. A: Overview showing the location of Sample OM09-2P-MS on top of a travertine terrace. B: A close-up view of peridotite cobbles within travertine. Sample OM09-2P-MS was collected from these cobbles. 


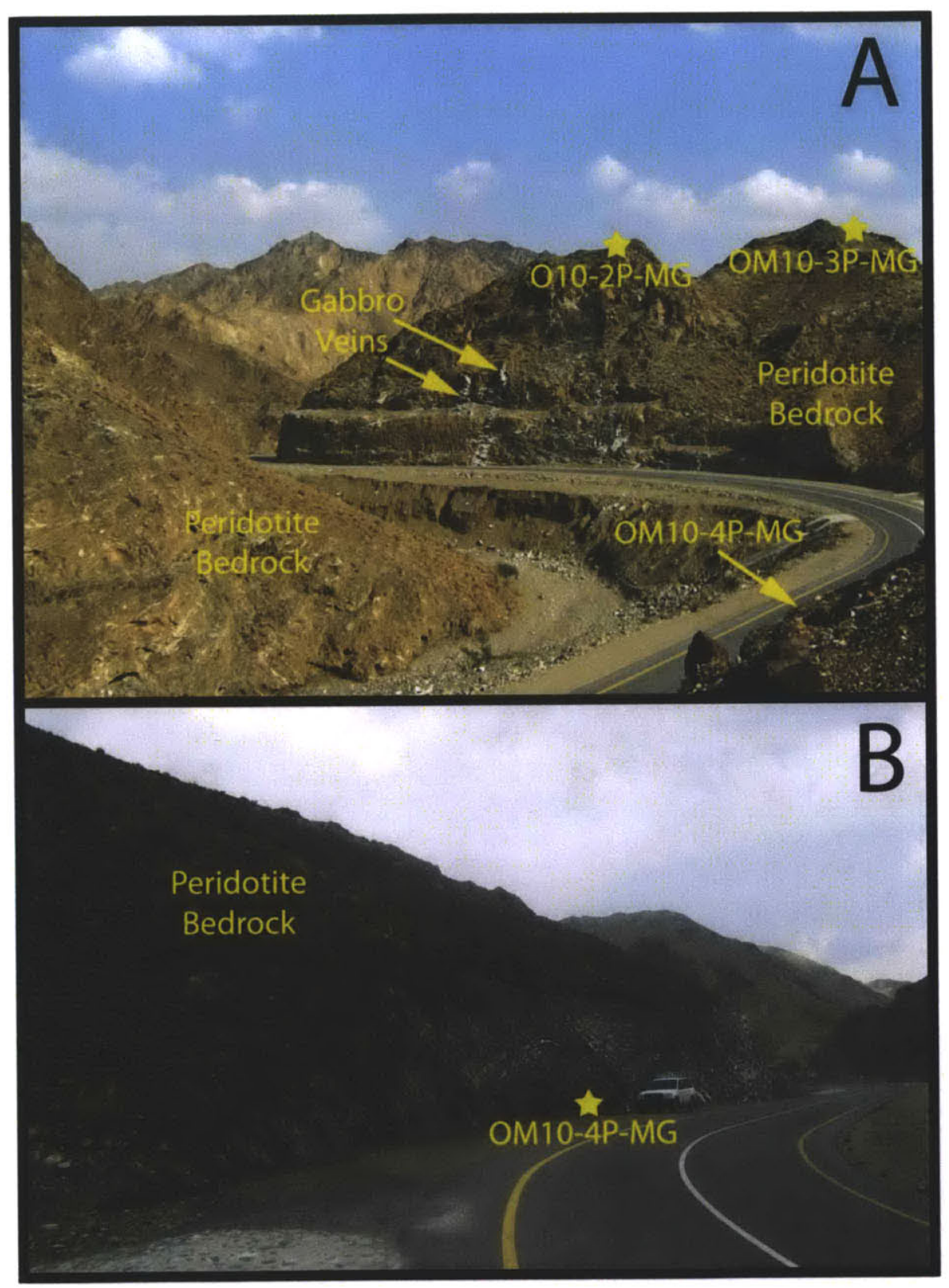

Figure 3: Fanja Roadcut. A: Overview showing the sampling locations for samples OM10-2P-MG and OM10-3P-MG on the natural peridotite weathering surface above a roadcut which exposes peridotite bedrock which contains veins of gabbro, serpentine, and carbonate. B: A view of another section of roadcut (location indicated in Panel A as well) at Fanja. Shielded sample OM10-4P-MG was collected from the bottom of this roadcut. 


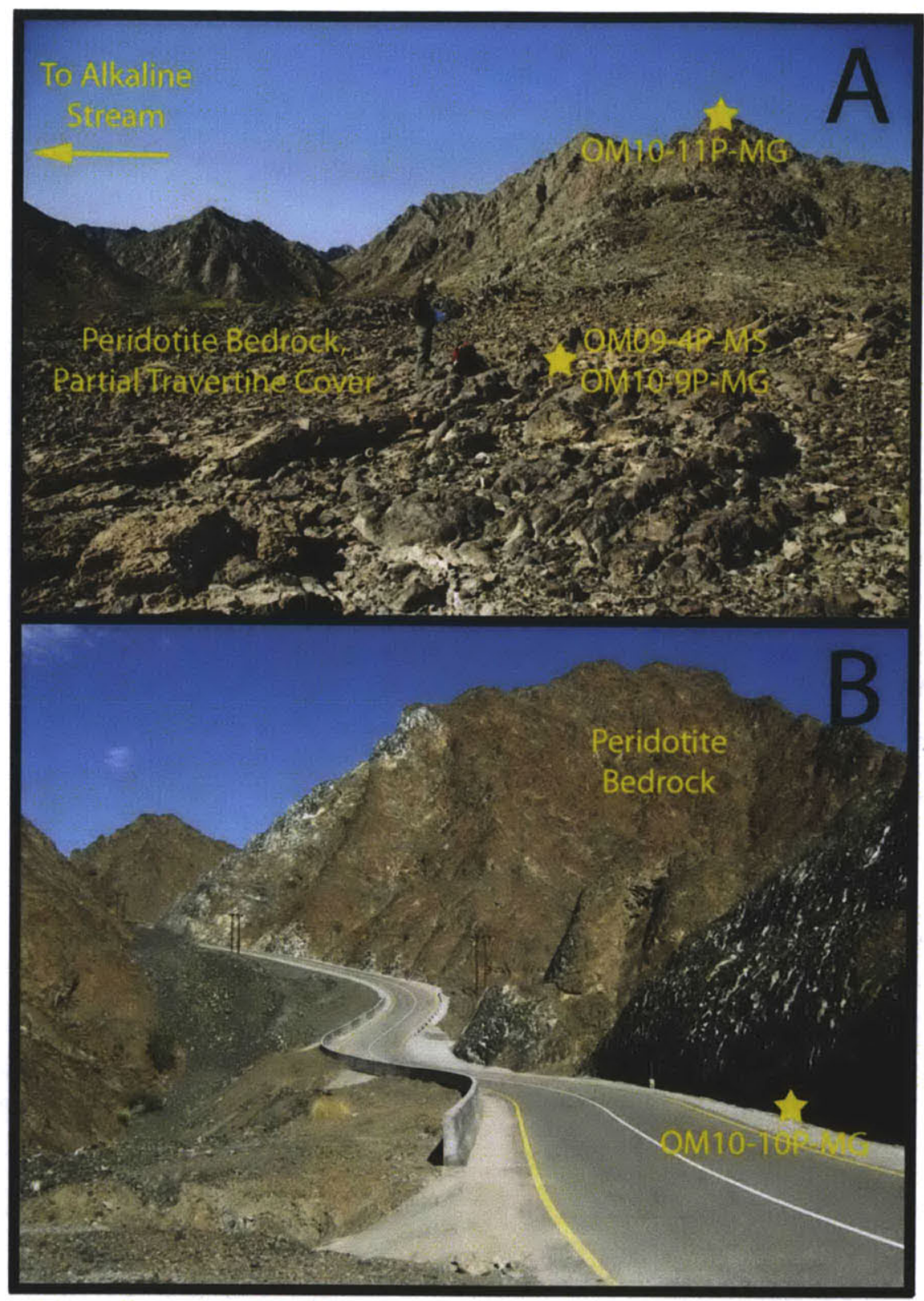

Figure 4: Qafeefah. A: Locations of peridotite sampling at Qafeefah Travertine. OM094P-MS and OM10-9P-MG were collected in the valley from peridotite bedrock which has been partially-covered by travertine. These samples were collected $\sim 15 \mathrm{~m}$ from the primary alkaline spring at Qafeefah. Sample OM10-11P-MG was collected from the top of a peridotite hill located next to the valley. B: A view of Qafeefah Roadcut $(\sim 2 \mathrm{~km}$ from Qafeefah Travertine), showing the sampling location for shielded sample OM1010P-MG. 


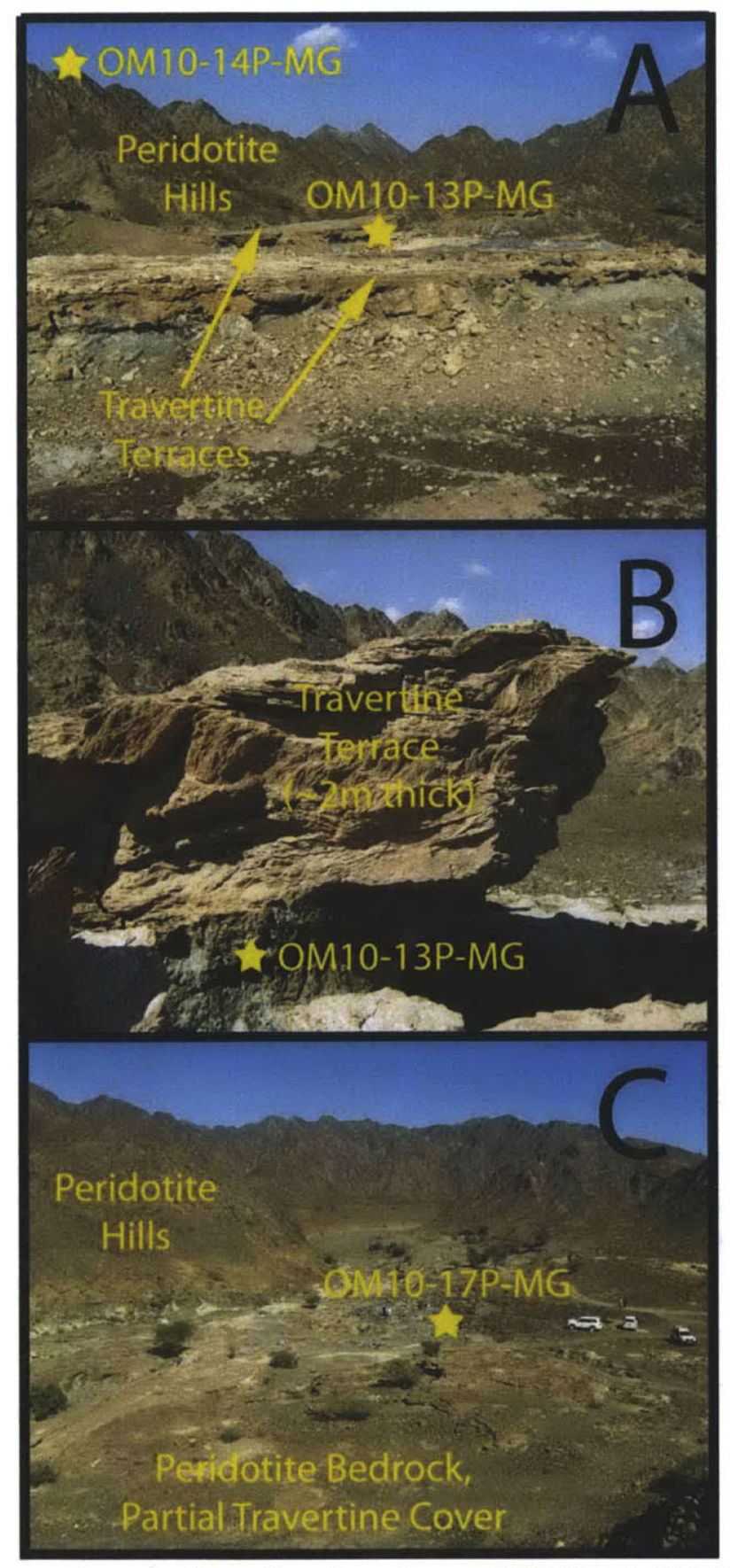

Figure 5: Misht Travertine. A: Sampling locations of OM10-14P-MG (top of peridotite hills) and shielded sample OM10-13P-MG (underneath a $\sim 2 \mathrm{~m}$ thick travertine terrace) at the northwest end of Misht Travertine. B: A close-up view of the sampling location for shielded sample OM10-14P-MG. C: Sampling location of OM10-17P-MG at the southeast end of the travertine deposit, which is located $\sim 2 \mathrm{~km}$ away from the sampling area shown in Panel A. 


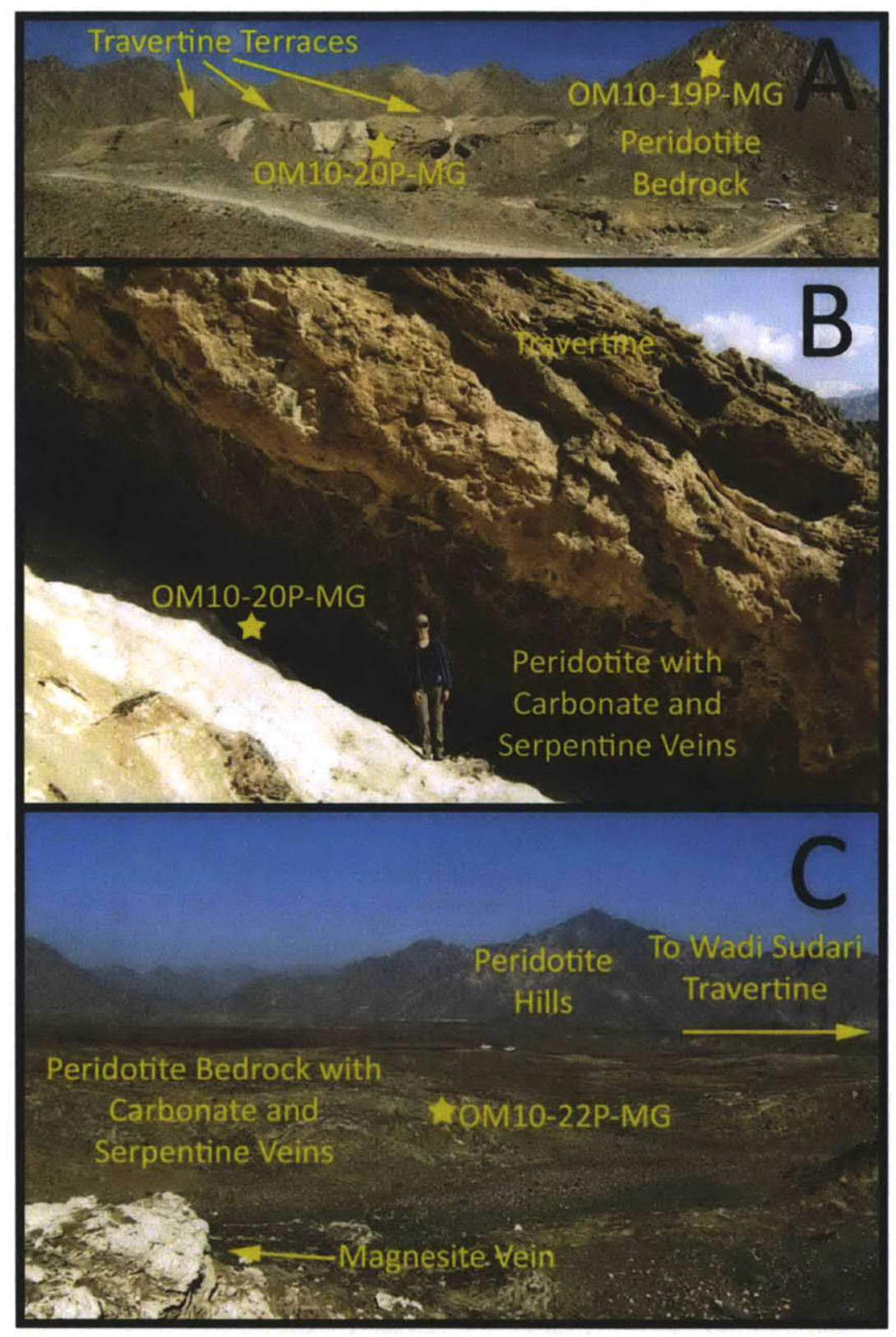

Figure 6: Wadi Sudari. A: Panorama of the large travertine deposit at Wadi Sudari showing the sampling locations of OM10-19P-MG (peridotite hills) and shielded sample OM10-20P-MG (from an overhang underneath a $\sim 2 \mathrm{~m}$ thick travertine terrace). B: A closer view of the sampling location for shielded sample OM10-20P-MG underneath a travertine terrace. C: Wadi Sudari campsite, a flat area of peridotite bedrock with abundant carbonate and serpentine veins located $\sim 2-3 \mathrm{~km}$ from Wadi Sudari travertine. Sample OM10-22P-MG was collected from the middle of this campsite. 


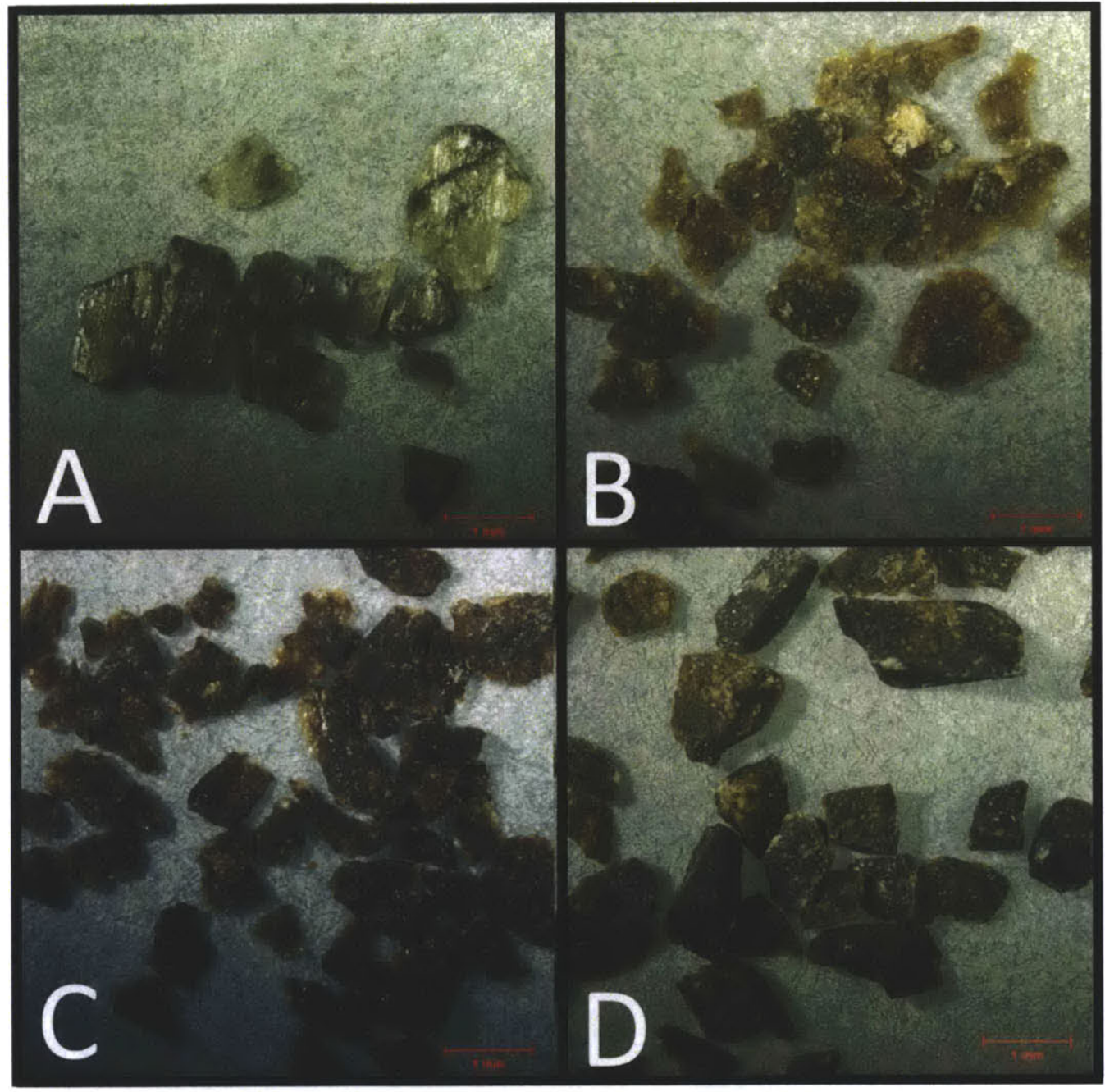

Figure 7: Representative images of mineral grains analyzed for helium concentrations and isotopes. $1 \mathrm{~mm}$ scalebars are indicated in red. All grains have experienced some alteration and/or serpentinization. A: Olivine mineral separate for sample OM10-22PMG. B: Pyroxene mineral separate for sample OM10-14P-MG. C: Altered olivine mineral separate for sample OM10-4P-MG. D: Whole rock grains for sample OM10-11PMG. 


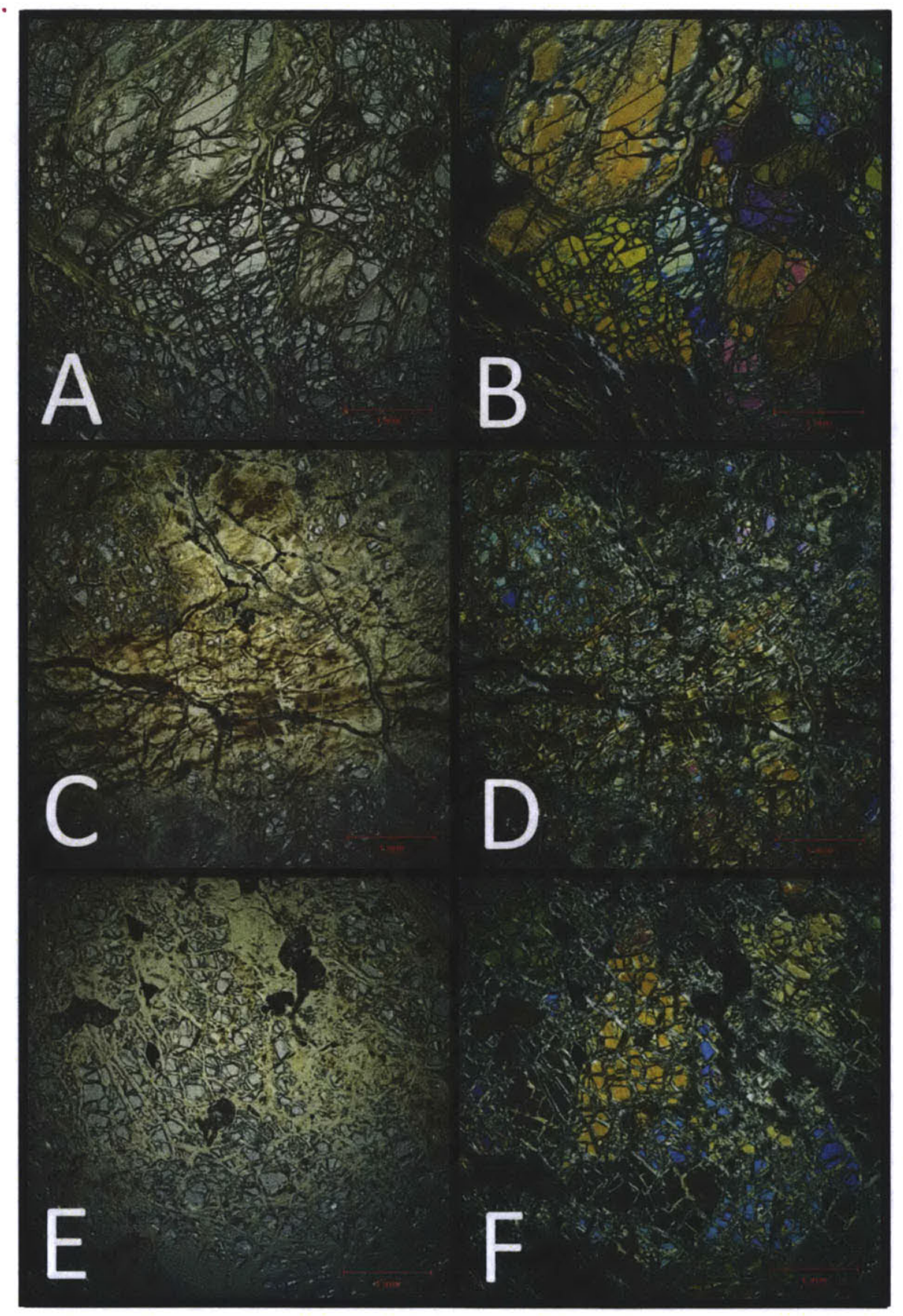

Figure 8: Representative thin section pictures in plane polarized light (lefthand side) and cross polarized light (righthand side). $1 \mathrm{~mm}$ scalebars are indicated in red. A and B: Thin section images for OM10-14P-MG, a $\sim 40 \%$ serpentinized harzburgite. C and D: Thin section images for OM10-9P-MG, a $\sim 90 \%$ serpentinized harzburgite. $\mathrm{E}$ and $\mathrm{F}$ : Thin section images for OM10-4P-MG, a $\sim 30 \%$ serpentinized dunite. 


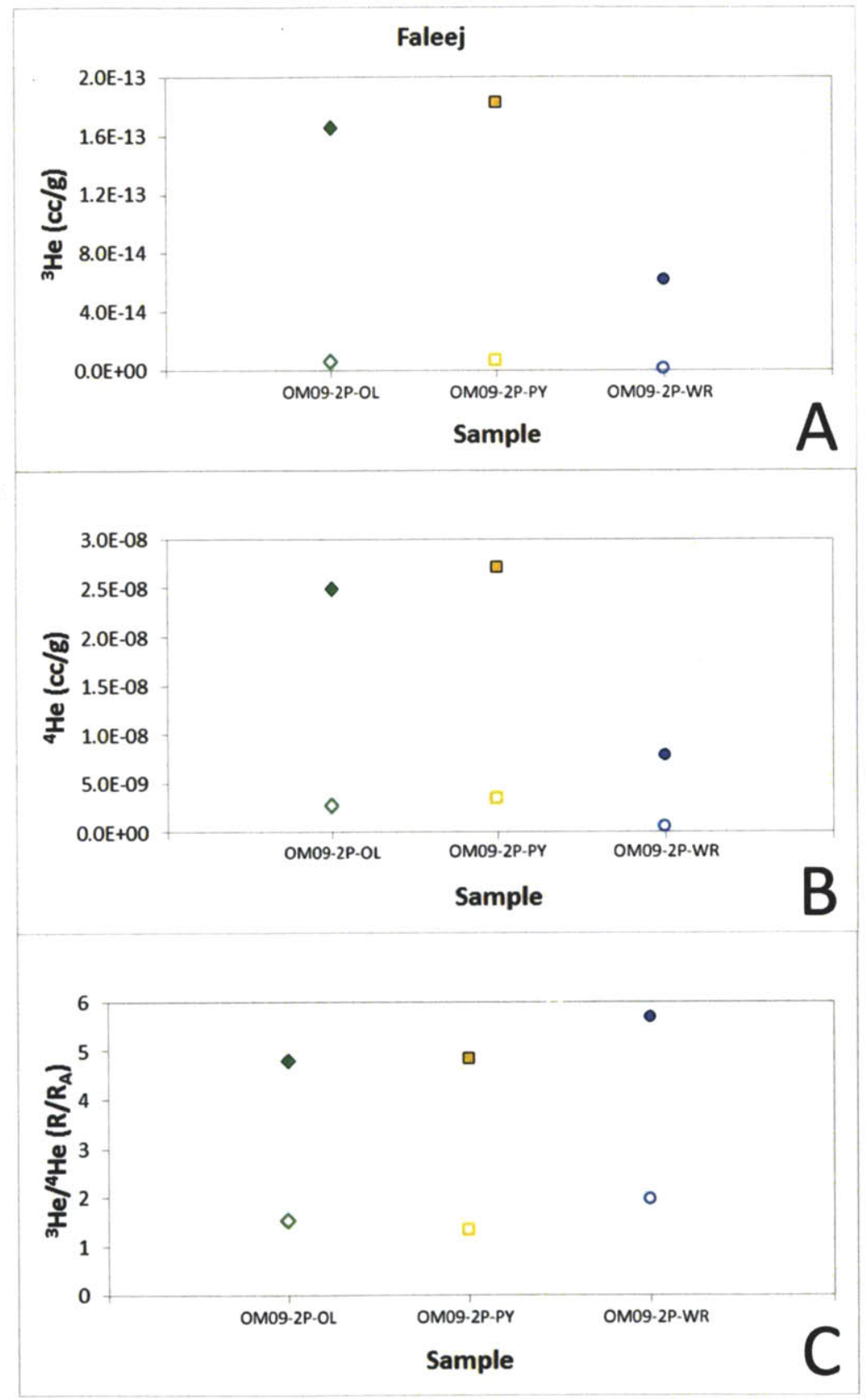

Figure 9: Plots of ${ }^{3} \mathrm{He}(\mathrm{A}),{ }^{4} \mathrm{He}(\mathrm{B})$, and ${ }^{3} \mathrm{He} /{ }^{4} \mathrm{He}$ (C) data versus sample for Faleej. The open symbols are crush values and the closed symbols are melt values. At Faleej there are three separates from the same unshielded rock, OM09-2P-MS. OM09-2P-OL is an olivine separate, OM09-2P-PY is a pyroxene separate, and OM09-2P-WR is a whole rock fraction. 


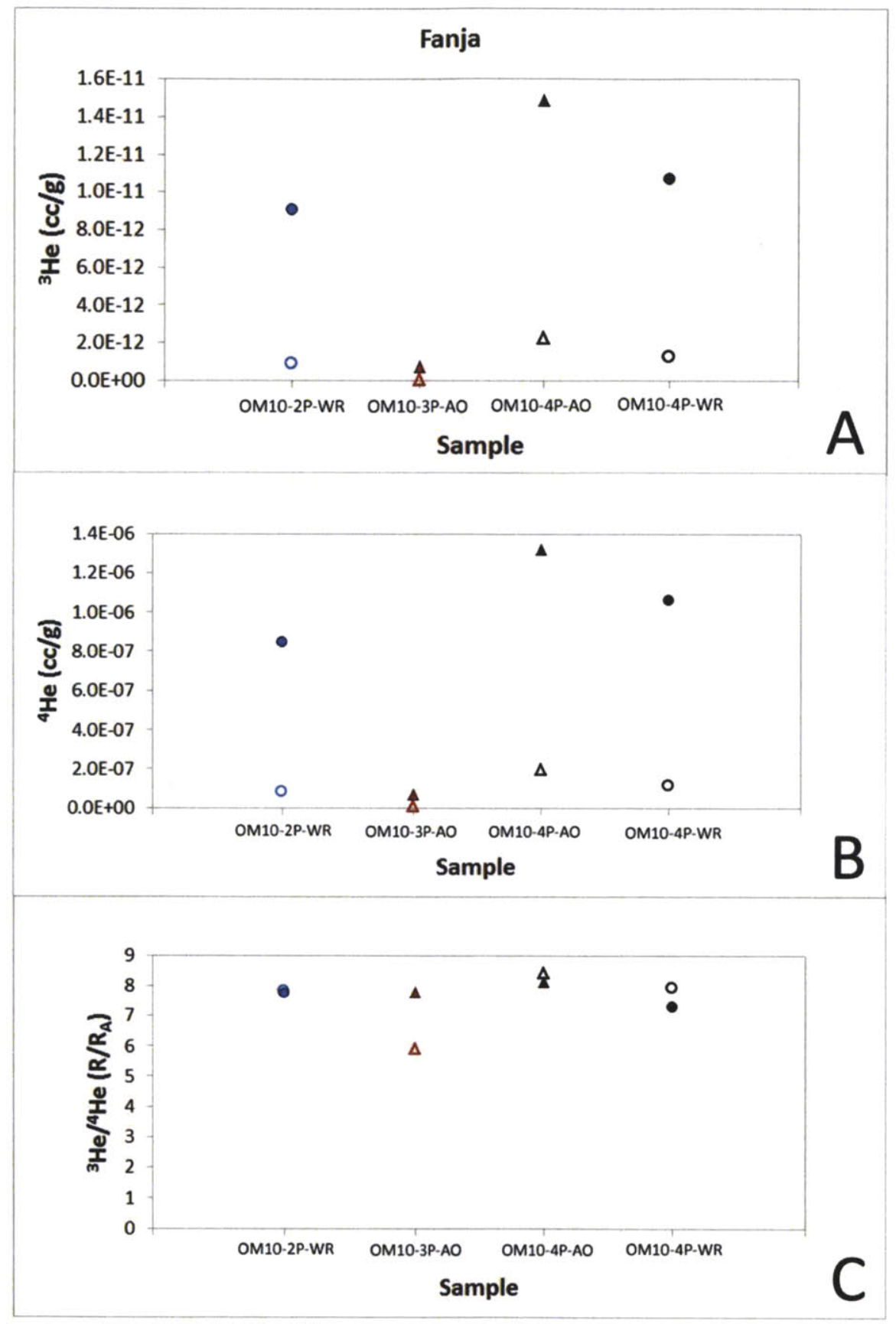

Figure 10: Plots of ${ }^{3} \mathrm{He}(\mathrm{A}),{ }^{4} \mathrm{He}(\mathrm{B})$, and ${ }^{3} \mathrm{He} /{ }^{4} \mathrm{He}(\mathrm{C})$ data versus sample for Fanja. The open symbols are crush values and the closed symbols are melt values. The colored symbols indicate unshielded samples while the black symbols indicate shielded samples. OM10-2P-WR and OM0-4P-WR are whole rock fractions and OM10-3P-AO and OM10$4 \mathrm{P}-\mathrm{AO}$ are altered olivine separates. 


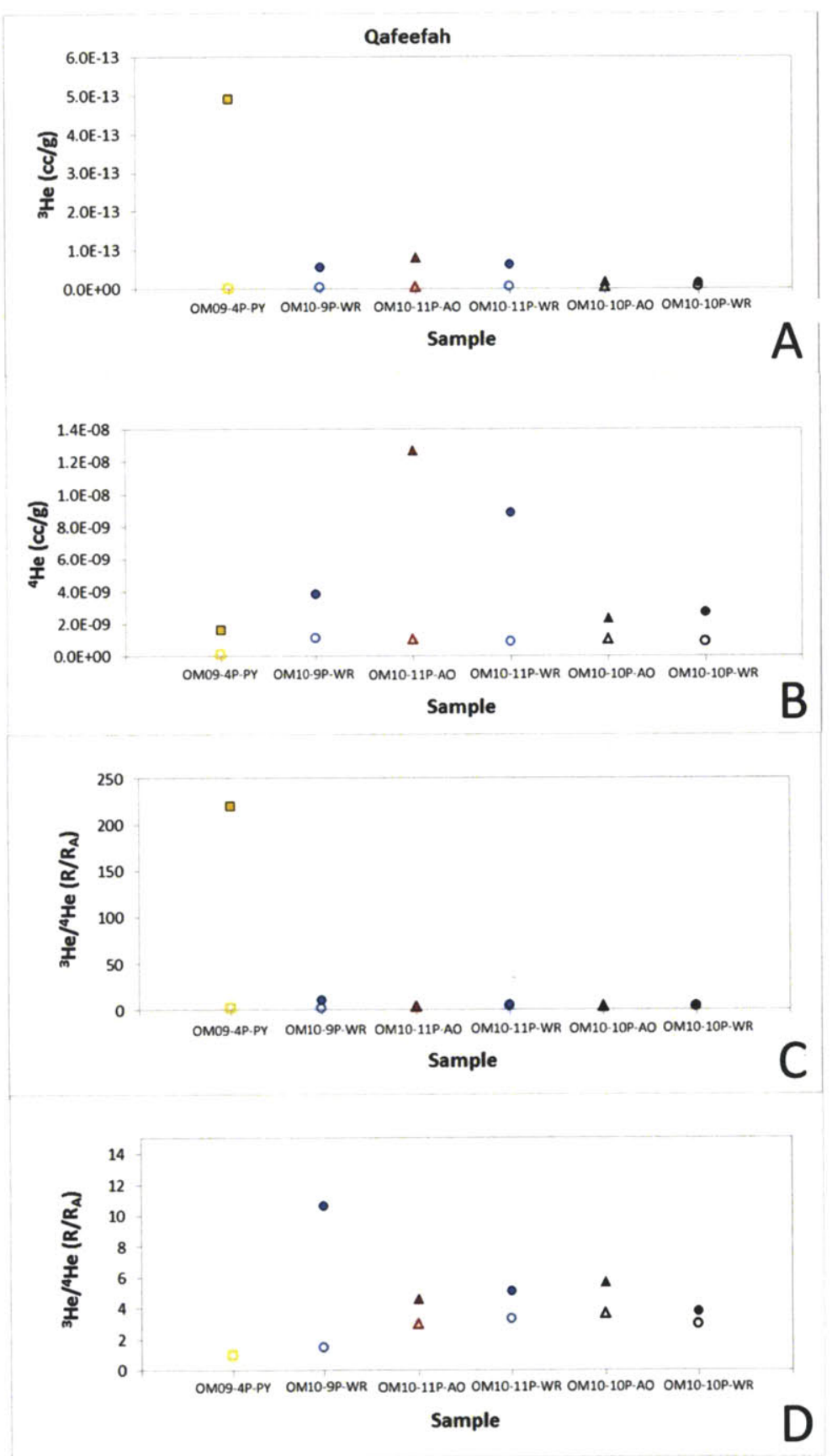

Figure 11: Plots of ${ }^{3} \mathrm{He}(\mathrm{A}),{ }^{4} \mathrm{He}(\mathrm{B})$, and ${ }^{3} \mathrm{He} /{ }^{4} \mathrm{He}(\mathrm{C})$ data versus sample for Qafeefah. Panel D is a zoom in of Panel C. The open symbols are crush values and the closed symbols are melt values. The colored symbols indicate unshielded samples while the black symbols indicate shielded samples. OM09-4P-PY is a pyroxene separate; OM109P-WR, OM10-11P-WR, and OM10-10P-WR are whole rock fractions; and OM10-11P$\mathrm{AO}$ and OM10-10P-AO are altered olivine separates. 

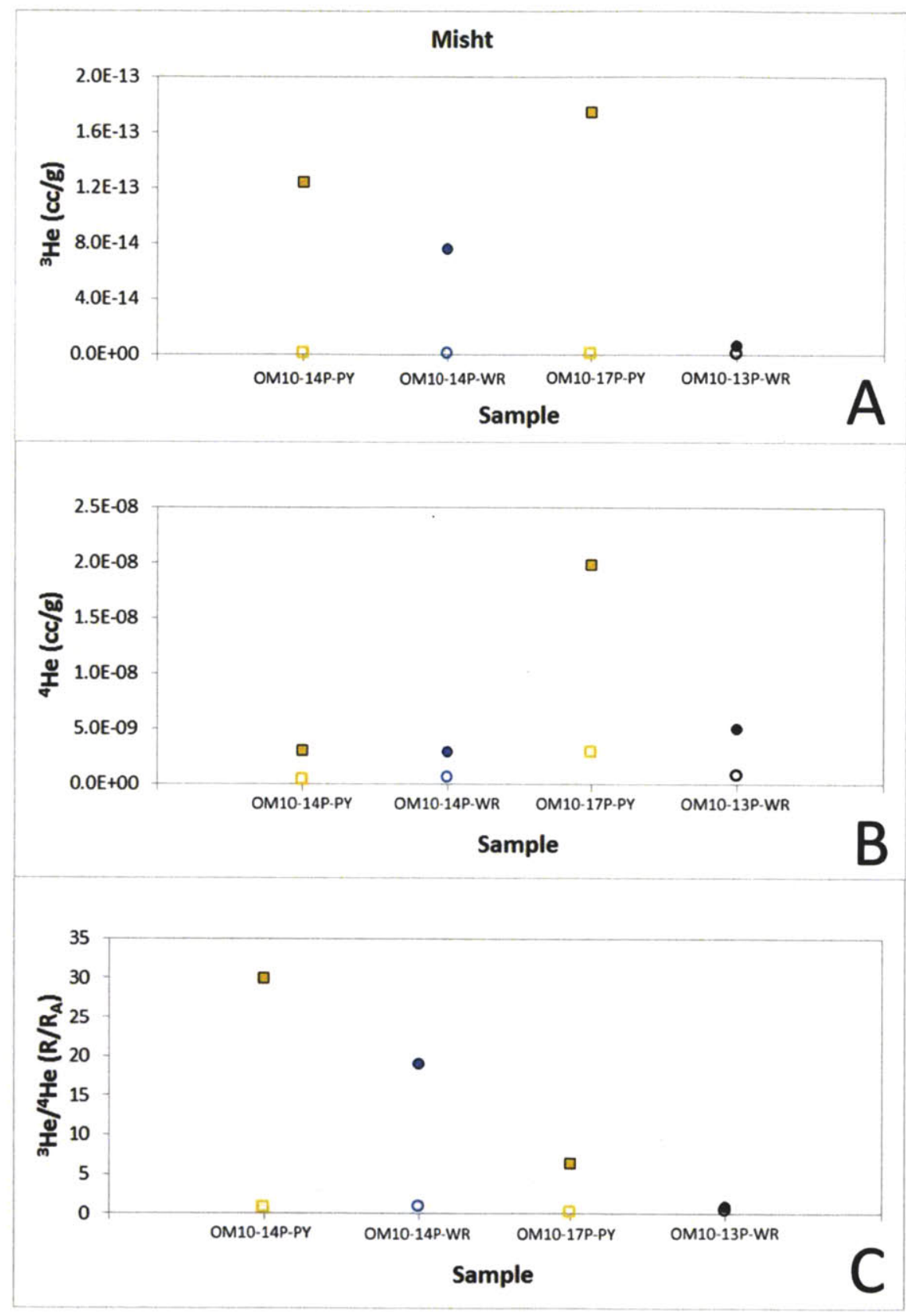

Figure 12: Plots of ${ }^{3} \mathrm{He}(\mathrm{A}),{ }^{4} \mathrm{He}(\mathrm{B})$, and ${ }^{3} \mathrm{He} /{ }^{4} \mathrm{He}(\mathrm{C})$ data versus sample for Misht. The open symbols are crush values and the closed symbols are melt values. The colored symbols indicate unshielded samples while the black symbols indicate shielded samples. OM10-14P-PY and OM10-17P-PY are pyroxene separates and samples OM10-14P-WR and OM10-13P-WR are whole rock fractions. 


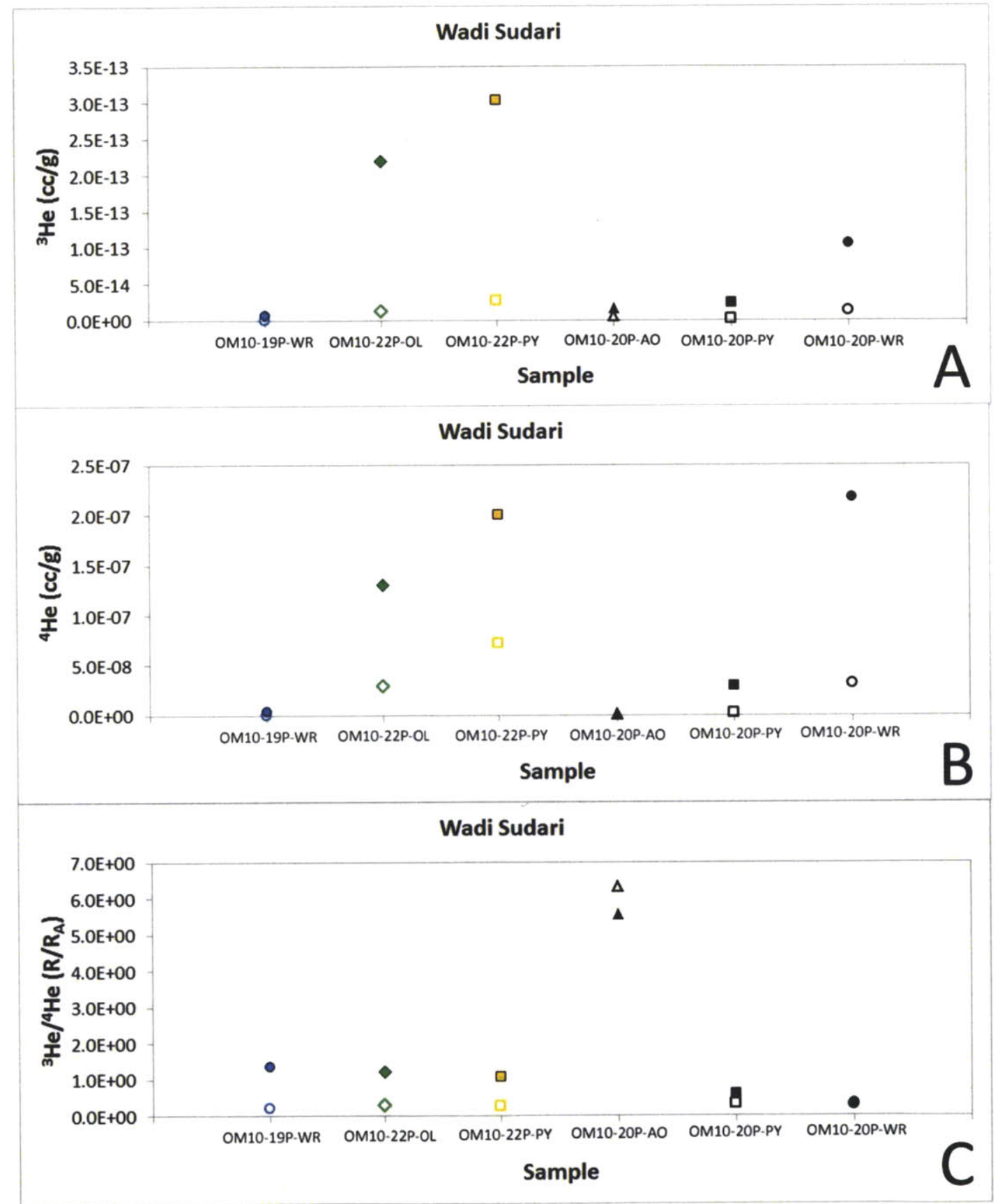

Figure 13: Plots of ${ }^{3} \mathrm{He}(\mathrm{A}),{ }^{4} \mathrm{He}(\mathrm{B})$, and ${ }^{3} \mathrm{He} /{ }^{4} \mathrm{He}(\mathrm{C})$ data versus sample for Wadi Sudari. Panel D is a zoom in of Panel C. The open symbols are crush values and the closed symbols are melt values. The colored symbols indicate unshielded samples while the black symbols indicate shielded samples. OM10-19P-WR and OM10-20P-WR are whole rock fractions; OM10-22P-OL is an olivine separate; OM10-22P-PY and OM1020P-PY are pyroxene fractions; and OM10-20P-AO is an altered olivine separate. 


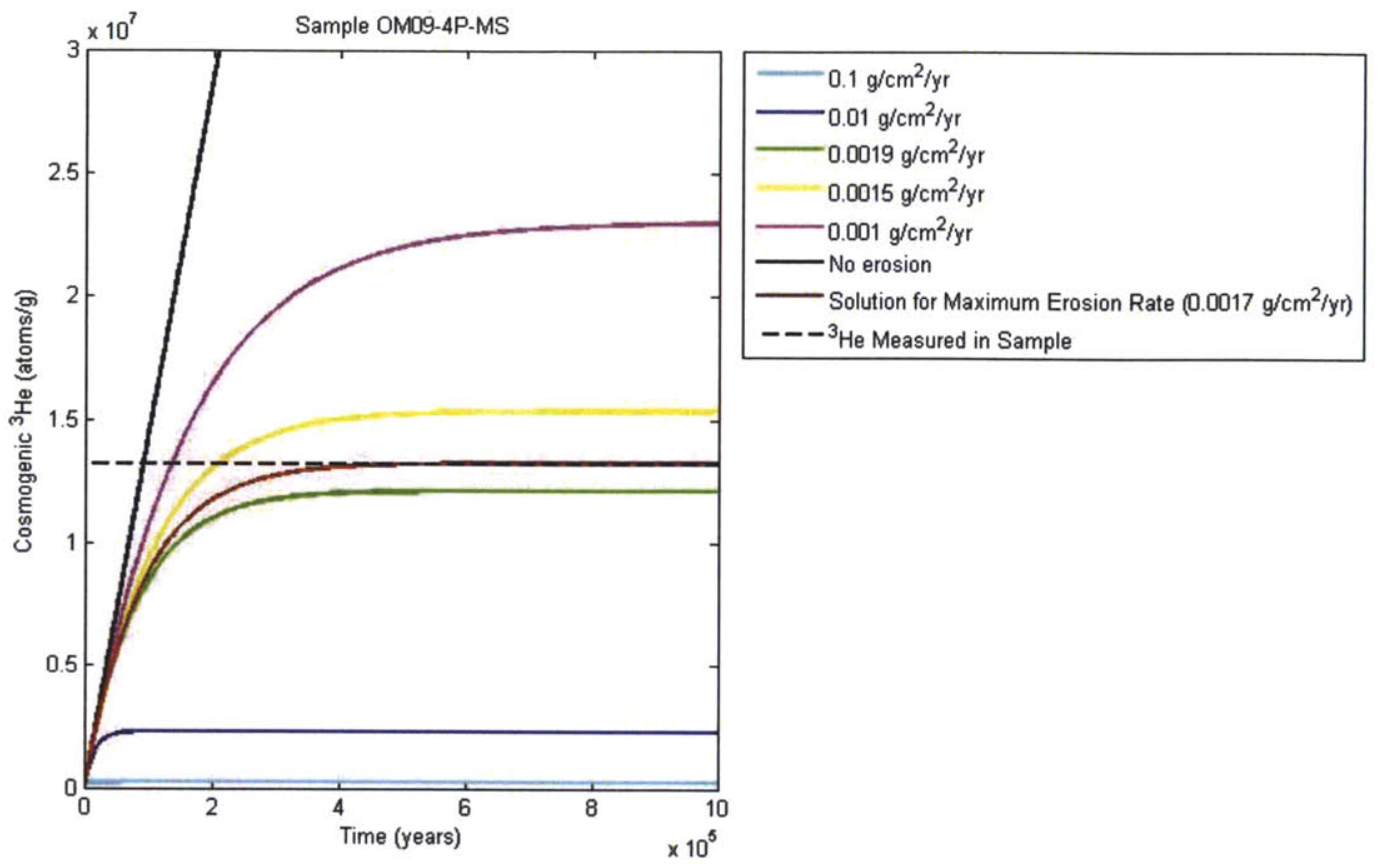

Figure 14: Calculation of maximum erosion rates using Equation 3 for Sample OM09-4PMS. The black line shows the solution for Equation 3 assuming no erosion. The intersection of this black line with the dashed line, which represents the cosmogenic ${ }^{3} \mathrm{He}$ in sample OM09-4P-MS (the total ${ }^{3} \mathrm{He}$ is plotted here, but for this sample cosmogenic ${ }^{3} \mathrm{He}$ determined by assumptions \#2 and \#3 are very similar), gives the minimum exposure age for this sample. The maximum erosion rate is calculated by plotting solutions to Equation 3 for various erosion rates. The erosion rate which plateaus at a ${ }^{3} \mathrm{He}$ value equal to the cosmogenic ${ }^{3} \mathrm{He}$ in the sample (red line) represents a maximum erosion rate for this sample. 


\section{References:}

Aka, F.T., Nagao, K., Kusakabe, M., and Nfomou, N. 2009. Cosmogenic helium andneon in mantle xenoliths from the Cameroon Volcanic Line (West Africa):Preliminary observations. Journal of African Earth Sciences, Vol. 55: 175-184.

Andrews, J.N. 1985. The isotopic composition of radiogenic helium and its use to studygroundwater movement in confined aquifers. Chemical Geology, Vol. 49: 339351.

Anslow, F.S., Clark, P.U., Kurz, M.D., and Hostetler, S.W. 2010. Geochronology and paleoclimatic implications of the last deglaciation of the Mauna Kea Ice Cap, Hawaii. Earth and Planetary Science Letters, Vol. 297: 234-248.

Barnes, I., LaMarche, V.C., and Himmelberg, G. 1967. Geochemical evidence of present day serpentinization. Science, Vol. 156: 830-832.

Barnes, I. and O'Neil, J.R. 1969. The relationship between fluids in some fresh Alpine type ultramafics and possible modern serpentinization, Western United States. Geological Society of America Bulletin, Vol. 80: 1947-1960.

Barnes, I., O’Neil, J.R., and Trescases, J.J. 1978. Present-day serpentinization in New Caledonia, Oman, and Yugoslavia. Geochimica et Cosmochimia Acta, Vol. 42: 144-145.

Beauvais, A., Parisot, J.-C., and Savin, C. 2007. Ultramafic rock weathering and slope erosion processes in a South West Pacific tropical environment. Geomorphology,Vol. 83: 1-13.

Blard, P.-H., Bourlès, D., Pik, R., and Lavé, J. 2008. In situ cosmogenic ${ }^{10} \mathrm{Be}$ in olivines and pyroxenes. Quaternary Geochronology, Vol. 3: 196-205.

Chen, Y. and Brantley, S.L. 2000. Dissolution of forsteritic olivine at $65^{\circ} \mathrm{C}$ and $2<\mathrm{pH}$ $<5$. Chemical Geology, Vol. 165: 267-281.

Clapp, E.M., Bierman, P.R., Schick, A.P., Lekach, J., Enzel, Y., and Caffee, M. 2000. Sediment yield exceeds sediment production in arid drainage basins. Geology, Vol. 28, No. 11: 995-998.

Cook, S.J. and Fletcher, W.K. 1993. Distribution and behaviour of platinum in soils, sediments, and waters of the Tulameen ultramafic complex, southern British Columbia, Canada. Journal of Geochemical Exploration, Vol. 46: 279-308. 
Dzemua, G. L., and Gleeson, S.A. 2012. Petrography, mineralogy, and geochemistry of the Nkamouna serpentinite: Implications for the formation of the cobalt manganese laterite deposit, southeastern Cameroon. Economic Geology, Vol. 107: $25-41$.

Evans, B.W. 1977. Metamorphism of alpine peridotite and serpentinite. Annual Reviews of Earth and Planetary Sciences, Vol. 5: 397-447.

Farley, K.A. and Neroda, E. 1998. Noble gases in the Earth's mantle. Annual Reviews of Earth and Planetary Sciences, Vol. 26: 189-218.

Freyssinet, P. and Farah, A. S. Geochemical mass balance and weathering rates of ultramafic schists in Amazonia. Chemical Geology, Vol. 170: 133-151.

Frost, B.R. 1985. On the stability of sulfides, oxides, and native metals in serpentinite. Journal of Petrology, Vol. 26: 31-63.

Gautheron, C. and Moreira, M. 2002. Helium signature of the subcontinental lithospheric mantle. Earth and Planetary Science Letters, Vol. 199: 39-47.

Gautheron, C., Moreira, M., and Allègre, C. 2005. He, Ne, and Ar composition of the European lithospheric mantle. Chemical Geology, Vol. 217: 97-112.

Giammar, D.E., Bruant, R.G., and Peters, C.A. 2005. Forsterite dissolution and magnesite precipitation at conditions relevant for deep saline aquifer storage and sequestration of carbon dixide. Chemical Geology, Vol. 217: 257-276.

Gleeson, S.A., Butt, C.R.M., and Elias, M. 2003. Nickel laterites: A review. Society of Economic Geologists Newsletter, Vol. 54, No. 1: 12-18.

Goehring, B.M., Kurz, M.D., Balco, G., Schaefer, J.M., Liccardi, J., and Lifton, N. 2010. A reevaluation of in situ cosmogenic ${ }^{3} \mathrm{He}$ production rates. Quaternary Geochronology, Vol. 5: 410-418.

Golightly, J.P. 1981. Nickeliferous laterite deposits. Economic Geology $75^{\text {th }}$ Anniversary Volume: 710-735.

Golightly, J.P. 2010. Progress in understanding the evolution of nickel laterites. Society of Economic Geologists Special Publication, Vol. 15: 451-486.

Hänchen, M., Prigiobbe, V., Storti, G., Seward, T.M., and Mazzotti, M. 2006. Dissolution kinetics of forsteritic olivine at $90-150^{\circ} \mathrm{C}$ including effects of the presence of $\mathrm{CO}_{2}$. Geochimica et Cosmochimica Acta, Vol. 70: 4403-4416. 
Hanson, E.K., Moore, J.M., Bordy, E.M., Marsh, J.S., Howarth, G., and Robey, J.V.A. 2009. Cretaceous erosion in central South Africa: Evidence from upper-crustal xenoliths in kimberlite diatremes. South African Journal of Geology, Vol. 112: $125-140$.

Hilton, D.R., Grönvold, K., Macpherson, C.G., and Castillo, P.R. 1999. Extreme ${ }^{3} \mathrm{He} /{ }^{4} \mathrm{He}$ ratios in northwest Iceland: constraining the common component in mantle plumes. Earth and Planetary Science Letters, Vol. 173: 53-60.

Kelemen, P.B. and Matter, J.M. 2008. In situ carbonation of peridotite for $\mathrm{CO}_{2}$ storage. Proceedings of the National Academy of Sciences, Vol. 105, No.45: 1729517300.

Kelemen, P.B., Matter, J.M., Streit, E.E., Rudge, J.F., Curry, W.B., and Blusztajn, J. 2011. Rates and mechanisms of mineral carbonation in peridotite: Natural processes and recipes for enhanced, in situ $\mathrm{CO}_{2}$ capture and storage. Annual Reviews of Earth and Planetary Sciences, Vol. 39: 545-576.

Kumagai, H., Dick, H.J.B., and Kaneoka, I. Noble gas signatures of abyssal gabbros and peridotites at an Indian Ocean core complex. Geochemistry, Geophysics Geosystems, Vol. 4, No. 12: 16 pp.

Kurz, M. D. 1986a. Cosmogenic helium in a terrestrial igneous rock. Nature, Vol. 320: 435-439.

Kurz, M.D. 1986b. In situ production of terrestrial cosmogenic helium and some applications to geochronology. Geochimica et Cosmochimica Acta, Vol. 50: 2855-2862.

Kurz, M.D., Colodner, D., Trull, T. W., Moore, R. B., and O’Brien, K. 1990. Cosmic ray exposure dating with in situ produced cosmogenic ${ }^{3} \mathrm{He}$ : results from young Hawaiian lava flows. Earth and Planetary Science Letters, Vol. 97: 177-189.

Kurz, M.D., Curtice, J., Lott, D.E., and Solow, A. 2004. Rapid helium isotopic variability in Mauna Kea shield lavas from the Hawaiian Scientific Drilling Project. Geochemistry, Geophysics, Geosystems, Vol. 5, No. 4: 35 pp.

Lackner, K.S., Wendt, C.H., Butt, D.P., Joyce, E.L., and Sharp, D.H. 1995. Carbon dioxide disposal in carbonate minerals. Energy, Vol. 20: 1153-1170.

Lal, D. 1991. Cosmic ray labeling of erosion surfaces: in situ nuclide production rates and erosion models. Earth and Planetary Science Letters, Vol. 104: 424-439. 
Llorca, S. and Monchoux, P. 1991. Supergene cobalt minerals from New Caledonia. Canadian Mineralogist, Vol. 29: 149-161.

Luce, R. W., Bartlett, R. W., and Parks, G.A. 1972. Dissolution kinetics of magnesium silicates. Geochimica et Cosmochimica Acta, Vol. 36: 35-50.

Matter, J.M. and Kelemen, P.B. 2009. Permanent storage of carbon dioxide in geological reservoirs by mineral carbonation. Nature Geoscience, Vol. 2: 837-841.

Metz, B., Davidson, O., de Coninck, H., Loos, M., and Meyer, L. (Eds.), 2005. IPCC Special Report on Carbon Dioxide Capture and Storage. New York: Cambridge University Press, pp. 431.

Moreira, M., Kunz, J., and Allègre, C.J. 1998. Rare gas systematics in popping rock: Isotopic and elemental compositions in the upper mantle. Science, Vol. 279:11781181 .

Moreira, M. and Madureira, P. 2005. Cosmogenic helium and neon in 11 Myr old ultramafic xenoliths: Consequences for mantle signatures in old samples. Geochemistry, Geophysics, Geosystems, Vol. 6, No. 8: 5 pp.

Moretti, I. and Turcotte, D.L. 1985. A model for erosion, sedimentation, and flexure with application to New Caledonia. Journal of Geodynamics, Vol. 3: 155-168.

Morrison, P. and Pine, J. 1955. Radiogenic origin of the helium isotopes in rock. Annals of the New York Academy of Sciences, Vol. 62, No. 3: 69-92.

Pokrovsky, O.S. and Schott, J. 2000. Kinetics and mechanism of foresterite dissolution at $25^{\circ} \mathrm{C}$ and $\mathrm{pH}$ from 1 to 12. Geochimica et Cosmochimica Acta, Vol. 64, No. 19: 3313-3325.

Porcelli, D.R., Stone, J.O.H., and O'Nions, R.K. 1987. Enhanced ${ }^{3} \mathrm{He} /{ }^{4} \mathrm{He}$ ratios and cosmogenic helium in ultramafic xenoliths. Chemical Geology, Vol. 64: 25-33.

Portenga, E.W. and Bierman, P.R. 2011. Understanding Earth's eroding surface with ${ }^{10}$ Be. GSA Today, Vol. 21, No. 8: 4-10.

Prigiobbe, V., Costa, G., Baciocchi, R., Hänchen, M., and Mazzotti, M. 2009. The effect of $\mathrm{CO}_{2}$ and salinity on olivine dissolution kinetics at $120^{\circ} \mathrm{C}$. Chemical Engineering Science, Vol. 64: 3510-3515.

Recanati, A., Kurz, M.D., Warren, J.M., and Curtice, J. 2012. Helium distribution in a mantle shear zone from the Josephine Peridotite. Submitted to Earth and Planetary Science Letters. 
Rioux, M., Bowring, S.A., and Kelemen, P.B. 2008. Timescales of crusal accretion at a medium to fast spreading ridge: High precision $\mathrm{U}-\mathrm{Pb}$ zircon dating of the intrusive crust of the Cretaceous Oman Ophiolite. Eos, Transactions, American Geophysical Union, Vol. 89, No. 53, Supplement, Abstract V44B-02.

Seifritz, W. 1990. $\mathrm{CO}_{2}$ disposal by means of silicates. Nature, Vol. 345: 486.

Staudacher, Th., Sarda, Ph., Richardson, S.H., Allègre, C.J., Sanga, I., and Dmitriev, L. 1989. Noble gases in basalt glasses from a Mid-Atlantic Ridge topographic high at $14^{\circ} \mathrm{N}$ : geodynamic consequences. Earth and Planetary Science Letters, Vol. 96: 119-133.

Stuart, F.M., Lass-Evans, S., Fitton, J.G., and Ellam, R.M. 2003. High ${ }^{3} \mathrm{He} /{ }^{4} \mathrm{He}$ ratios in picritic basalts from Baffin Island and the role of a mixed reservoir in mantle plumes. Nature, Vol. 424: 57-59.

Summons, T.G., Green, D.C., and Everard, J.L. 1981. The occurrence of chromite in the Anderson Creek area, Beaconsfield, Tasmania. Economic Geology, Vol. 76: 505518.

Tilton, G.R., Hopson, C.A., and Wright, J.E. 1981. Uranium-lead isotopic ages of the Samail Ophiolite, Oman, with applications to Tethyan Ocean Ridge Tectonics. Journal of Geophysical Research, Vol. 86, No. B4: 2763-2775.

Trommsdorff, V. and Evans, B.W. 1977. Antigorite-ophicarbonates: Contact metamorphism in Valmalenco, Italy. Contributions to Mineralogy and Petrology, Vol. 62: 301-312.

Trommsdorff, V., Evans, B.W., and Pfeifer, H.R. 1980. Ophicarbonate rocks: Metamorphic reactions and a possible origin. Archives des Sciences Genève, Vol. 33: 361-364.

Trull, T.W., Kurz, M.D., and Jenkins, W.J. 1991. Diffusion of cosmogenic ${ }^{3} \mathrm{He}$ in olivine and quartz: Implications for surface exposure dating. Earth and Planetary Science Letters, Vol. 103, No. 1-4: 241-256.

Trull, T.W. and Kurz, M.D. 1993. Experimental measurements of ${ }^{3} \mathrm{He}$ and ${ }^{4} \mathrm{He}$ mobility in olivine and clinopyrozene at magmatic temperatures. Geochimica et Cosmochimica Acta, Vol. 57: 1313-1324.

Van Herk, J., Pietersen, H.S., and Schuiling, R.D. 1989. Neutralization of industrial waste acids with olivine: The dissolution of forsteritic olivine at $40-70^{\circ} \mathrm{C}$. Chemical Geology, Vol. 76: 341-352. 
Wilson, S.A., Raudseep, M., and Dipple, G.M. 2006. Verifying and quantifying carbon fixation in minerals from serpentine-rich mine tailings using the Rietveld method with X-ray powder diffraction data. American Mineralogist, Vol. 91, No. 8-9: $1331-1341$.

Wilson, S.A., Raudsepp, M., and Dipple, G.M. 2009a. Quantifying carbon fixation in trace minerals from processed kimberlite: A comparative study of quantitative methods using X-ray powder diffraction data with applications to the Diavik Diamond Mine, Northwest Territories, Canada. Applied Geochemistry, Vol. 24, No. 12: 95-112.

Wilson, S.A., Dipple, G.M., Power, I.M., Thom, J.M., Anderson, R.G., Raudsepp, M., Gabites, J.E., and Southam, G. 2009b. Carbon dioxide fixation within mine wastes of ultramafic-hosted ore deposits: Examples from the Clinton Creek and Cassiar chrysotile deposits, Canada. Economic Geology, Vol. 104, No. 1: 95-112.

Wogelius, R.A. and Walther, J.V. 1991. Olivine dissolution at $25^{\circ} \mathrm{C}$ : Effects of $\mathrm{pH}, \mathrm{CO}_{2}$, and organic acids. Geochimica et Cosmochimica Acta, Vol. 55: 943-954.

Yokochi, R., Marty, B., Pik, R., and Burnard, P. 2005. High ${ }^{3} \mathrm{He} /{ }^{4} \mathrm{He}$ ratios in peridotite xenoliths from SW Japan revisited: Evidence for cosmogenic ${ }^{3} \mathrm{He}$ released by vacuum crushing. Geochemistry, Geophysics, Geosystems, Vol. 6, No. 1: 12 pp. 


\section{Chapter 5: Synthesis and Future Directions}

An important goal of this study was to further constrain the natural rates of formation of carbonate alteration products in the peridotite layer of the Samail Ophiolite in the Sultanate of Oman through age dating of various travertines and veins. Based on

${ }^{14} \mathrm{C}$ dating, we have demonstrated that for the time period $\sim 30,000-45,000 \mathrm{yr}$ BP an upper estimate $^{1}$ of $\sim 1,000-3,000 \mathrm{~m}^{3} / \mathrm{yr}$ of travertine was deposited, sequestering $\sim 1-3 \times 10^{6} \mathrm{~kg}$ $\mathrm{CO}_{2} / \mathrm{yr}$.

This study has also demonstrated that a significant number of Mg-rich carbonate veins $\left(\sim 40 \%\right.$ at roadcuts and $\sim 8 \%$ overall) are ${ }^{14} \mathrm{C}$ dead $(>50,000$ yr BP). While attempts were made to determine the ages of these older $\left({ }^{14} \mathrm{C}\right.$ dead $) \mathrm{Mg}$-rich carbonate veins using ${ }^{230} \mathrm{Th}$ dating, application of this method was limited due to the extremely low concentrations of $\mathrm{U}$ and $\mathrm{Th}$ and also the significant corrections required for incorporated detritus. However, several ${ }^{14} \mathrm{C}$ dead veins are older than the $\sim 350,000 \mathrm{yr}$ limit of ${ }^{230} \mathrm{Th}$ dating, and two veins are likely $>1$ million years in age (based on equilibrium in $\left.\left({ }^{234} \mathrm{U} /{ }^{238} \mathrm{U}\right)\right)$. This results in an estimate that recent and ongoing formation of $\mathrm{Mg}$-rich carbonate veins sequesters on the order of $\sim 10^{7} \mathrm{~kg} \mathrm{CO}_{2} / \mathrm{yr}$.

Using measurements of cosmogenic ${ }^{3} \mathrm{He}$, this study determined maximum erosion rates of $\sim 5-180 \mathrm{~m} / \mathrm{Myr}$ (average: $\sim 40 \mathrm{~m} / \mathrm{Myr}$ ) for partially-serpentinized peridotite bedrock that contains the carbonate veins. If these erosion rates are extrapolated throughout the entire ophiolite, erosion of $\mathrm{Mg}$-rich carbonate veins in peridotite bedrock removes a maximum of $\sim 10^{5}-10^{6} \mathrm{~kg} \mathrm{CO}_{2} / \mathrm{yr}$, which is an order of magnitude lower than

\footnotetext{
${ }^{1}$ Based on simultaneous deposition of travertine over the total travertine area estimate of Kelemen and Matter (2008).
} 
the amount of $\mathrm{CO}_{2}$ sequestered through formation of $\mathrm{Mg}$-rich carbonate veins. The fate of this eroded carbonate and whether significant amounts of $\mathrm{CO}_{2}$ are released to the hydrosphere and/or atmosphere remains an open question. Some of the $\mathrm{CO}_{2}$ may continue to be stored in carbonate-rich cements in peridotite conglomerates, which are found in wadis throughout the peridotite layer, and in the carbonate-rich alluvial fans, which surround the ophiolite.

Overall, the estimates of carbonate formation and erosion rates determined in this study indicate that the peridotite layer of the Samail Ophiolite is a natural sink for $\mathrm{CO}_{2}$. However, the natural rate of $\mathrm{CO}_{2}$ uptake in the peridotite layer of the Samail Ophiolite is small relative to the $\sim 3.5 \times 10^{13} \mathrm{~kg} \mathrm{CO}_{2} / \mathrm{yr}$ of anthropogenic emissions to the atmosphere (e.g. Friedlingstein et al., 2010). Therefore, rates of $\mathrm{CO}_{2}$ uptake in peridotite would have to be greatly enhanced in order to significantly offset anthropogenic $\mathrm{CO}_{2}$ emissions (e.g. Kelemen and Matter, 2008).

\section{Travertine Deposition:}

This study has significantly increased the number of dated travertine samples and has expanded the number of locations where travertines have been dated. Together with previous studies (Clark and Fontes, 1990; Clark et al., 1992; Kelemen and Matter, 2008; Kelemen et al., 2011; Kelemen et al., unpublished data), we have improved our understanding of the timescales and rates of travertine formation in the peridotite layer of the Samail Ophiolite.

Future directions for research on Samail travertines include: 
-Further constraining rates of travertine formation during more recent (younger than $30,000 \mathrm{yr} \mathrm{BP}$ ) time periods to better estimate natural carbonation rates. This could be accomplished by dating younger travertine terraces, if they exist, and by dating layered samples from incised channels. Ongoing rates of travertine deposition could be estimated by direct (e.g. measuring rates of deposition on a substrate or placing markers in an active travertine deposit) or indirect (e.g. counting growth bands, if they exist, or measuring water chemistry at two or more fixed points and using a mass balance calculation) methods (see more in Chapter 10 of Pentecost, 2005).

-Improving estimates of the area of travertine deposition, both the total depositional area and the area over which travertine is being deposited at a given time. Since the total travertine area estimate of Kelemen and Matter (2008) is based on detailed mapping in the southern third of the ophiolite that was extrapolated throughout the entire ophiolite, additional mapping of travertines in the northern two-thirds of the ophiolite would be especially helpful in refining the estimate of total travertine area. Potentially, a satellite/GIS approach could be used to map travertine deposits although this approach may be limited by the dark-colored dust that coats many of the older, weathered travertines and makes them blend in with the surrounding peridotite bedrock. In order to determine the areas over which travertine was deposited in the past, detailed field mapping supplemented by additional ${ }^{14} \mathrm{C}$ dating is required. Using a faster and lessexpensive ${ }^{14} \mathrm{C}$ dating method, such as the sealed tube graphite method, could aid in the dating of large numbers of travertines. 
-Determining rates of travertine erosion. Although we observe weathered, incised travertine deposits in the field, there is currently no estimate for the rate at which travertines are being eroded. Travertine erosion rates could be determined by employing a microerosion meter (e.g. High and Hanna, 1970; Spate et al., 1985; Cucchi et al., 2006) or through measuring cosmogenic nuclides such as ${ }^{36} \mathrm{Cl}$ (e.g. Dockhurn et al., 1991; Stone et al., 1996, 1998), ${ }^{10} \mathrm{Be}$ (e.g. Braucher et al., 2005), and ${ }^{14} \mathrm{C}^{2}$ (e.g. Handwerger et al., 1999) in travertine deposits which are well-exposed to cosmic rays.

-Further investigating the hypothesis of Clark and Fontes (1990) that travertine formation in Oman is episodic and related to climate. Although the overall distribution of travertine ${ }^{14} \mathrm{C}$ ages does not support climate-related episodic travertine formation, there is clear evidence both from dating of the subsampled travertine terrace from Misht and from field evidence for episodic and variable rates of travertine formation. The observations that older terraces are weathered and incised, and that younger travertines primarily deposit in incised channels, suggest that at some time period(s) in the past, travertines were extensively eroded. In particular, it would be useful to determine if travertines were eroded during the Holocene humid period $\sim 8,000-9,000$ yr BP. Additional ${ }^{14} \mathrm{C}$ dating of travertines, perhaps focused on more detailed sequences or travertines with clear field relations, could be useful in further constraining the rates of travertine formation during various time periods.

\footnotetext{
${ }^{2}$ Carbonate erosion rates could possibly be determined by measuring cosmogenic ${ }^{14} \mathrm{C}$ that was produced in situ in the carbonate rock, primarily via spallation of oxygen atoms. This in situ cosmogenic ${ }^{14} \mathrm{C}$ must be distinguished from ${ }^{14} \mathrm{C}$ originating from the atmosphere and other sources, such as biological material. Handwerger et al. (1999) argued that this can be done by cryogenic separation methods. However, further work is needed to refine the techniques in their preliminary study.
} 
-Investigating the potential role of biological organisms in travertine deposition and/or erosion. We have observed evidence of both macroscopic (fish and other organisms) and microscopic (biofilms covering parts of the recently-formed travertine deposits) organisms in the hyperalkaline pools. Everett Shock and co-workers have found evidence of both methanogens and methanotrophs in the Samail hyperalkaline springs (Everett Shock, personal communication, 2012). Further, the recently-formed travertine deposits have very light $\delta^{13} \mathrm{C}$ and $\delta^{18} \mathrm{O}$ values that are unusual for non-biological carbonates and sometimes consist of aragonite, which is often biologically precipitated. While several authors (Clark et al., 1992; Wilson et al., 2010; Kelemen et al., 2011) have proposed that kinetic fractionation effects explain the light isotope values of the recentlyformed travertine precipitates, it would be worthwhile to investigate if biology plays a role in travertine precipitation. Microbes have several different methods of $\mathrm{CO}_{2}$ uptake, which is a process that may influence how carbonates would form. There are five main pathways that are currently known, and they may have different isotopic signatures even at low temperatures (e.g. Havig et al., 2011). In the Samail hyperalkaline springs, there may be an opportunity to find links between microbial uptake of $\mathrm{CO}_{2}$ and the triggering of carbonate mineralization.

\section{Formation of Mg-Rich Carbonate Veins:}

Understanding when and under what circumstances the older Mg-rich carbonate veins formed is an important area of future research. Although the $U$ concentrations of the Mg-rich carbonates are low (generally $1 \mathrm{ppb}$ or less), it may be possible to date the older Mg-rich carbonate veins using U-Pb dating (e.g. Moorbath et al., 1987; Smith and 
Farquhar, 1989; Jahn and Cuvellier, 1994; Richards et al., 1998; Cole et al., 2005). If suitable fluid inclusions can be found in the Mg-rich carbonate veins, then comparison of the composition of fluid inclusions (e.g. Schandl and Naldrett, 1992; Goldstein, 2001) in young and old $\mathrm{Mg}$-rich carbonate veins could provide information on the temperatures of formation and the fluid composition from which the older veins precipitated. Potentially, the older Mg-rich carbonate veins formed under shallow, low-temperature conditions similar to the ongoing low-temperature formation of carbonates. However, Mg-rich carbonates form under a range of conditions. The temperature of formation of the ${ }^{14} \mathrm{C}$ dead Mg-rich carbonate veins could be constrained by using the clumped isotope technique (e.g. Eiler, 2007, 2011). Preliminary studies on Mg-rich carbonate veins with finite ${ }^{14} \mathrm{C}$ ages have indicated that these veins formed at ambient temperatures (Streit et al., accepted), and have demonstrated the applicability of this technique to these samples.

The young ${ }^{230} \mathrm{Th}$ ages (relative to corresponding ${ }^{14} \mathrm{C}$ ages) of $\mathrm{Mg}$-rich carbonate outcrop veins as well as the observed ${ }^{238} \mathrm{U}$ excesses in altered peridotites indicate that these carbonates may have experienced post-formation addition of $U$, possibly from a reducing serpentinization fluid. The Mg-rich carbonate veins may experience dissolution/reprecipitation during interaction with this U-bearing fluid. In addition, one dolomite roadcut vein (OM10-47C-MG) consists of a clear layer that was ${ }^{14} \mathrm{C}$ dead and an opaque layer that had a finite ${ }^{14} \mathrm{C}$ age of $32,643 \pm 525 \mathrm{yr}$ BP. The opaque outer part of the vein may represent recrystallization or diagenesis of the inner, clear, ${ }^{14} \mathrm{C}$ dead part of the vein. The potential for open-system behavior in the Mg-rich carbonate veins should be further evaluated. Potentially, areas of recrystallization or diagenesis in Mg-rich 
carbonate veins could be identified microscopically, aided by cathodoluminescence analysis (e.g. ten Have and Heijen, 1985; Richter et al., 2003).

Another important area of future research is better constraining the depth of the weathering zone in which the Mg-rich carbonate veins (and also Ca-rich carbonate veins associated with the surface travertines) are forming via ongoing, low-temperature alteration of partially-serpentinized peridotite. One excellent way to investigate this would be through drilling and coring in the peridotite bedrock to evaluate the proportions and ages of the carbonate veins with depth. Alternatively, the depth of the peridotite weathering zone could be indirectly evaluated through additional measurements of alkaline spring water temperatures (e.g. Neal and Stanger, 1985), together with estimates of heat flow, and sampling of waters (and possibly carbonate?) from deep wells and falaj (water channel) tunnels.

\section{Beyond the Samail Ophiolite:}

Constraining rates of ultramafic weathering and carbonation is essential in order to fully understand and balance the global carbon cycle and also to evaluate the viability of using artificially-enhanced weathering of mafic and ultramafic rocks to mitigate the buildup of anthropogenic $\mathrm{CO}_{2}$ input to the atmosphere (e.g. Seiftritz, 1990; Lackner et al., 1995; Lackner, 2002; Kelemen and Matter, 2008; Matter and Kelemen, 2009; Gislason et al., 2010; Kelemen et al., 2011).

Significant ophiolite belts are found on the east coast and west coat of the United States, in central, eastern, and northern Europe, in the Middle East, in China, in Japan, in Papua New Guinea and elsewhere in Indonesia, and in eastern South America (c.g. 
Coleman, 1977; Dilek and Furnes, 2011). Overall, ophiolites cover $\sim 1 \%$ of the total continental surface (this is an approximate estimate based on the world ophiolite map in Coleman, 1977). Quantitative bedrock analysis provides more precise determinations of ultramafic bedrock exposure for certain regions: $0.15 \%$ for the conterminous United States (Peucker-Ehrenbrink and Miller, 2002); 0.20\% for Alaska (Peucker-Ehrenbrink and Miller, 2003); $0.08 \%$ for Canada (Peucker-Ehrenbrink and Miller, 2004); $0.9 \%$ for east and southeast Asia (Peucker-Ehrenbrink and Miller, 2004), and 1-2\% for Brazil (Peucker-Ehrenbrink and Miller, 2007).

Rates of carbonate formation and erosion in the peridotite layer of the Samail Ophiolite likely represent slow end-members since the climate in Oman is extremely arid relative to other parts of the world. The arid climate of Oman may be important for the preservation of carbonates as significant travertine deposits are not commonly observed at other ophiolites (Peter Kelemen, personal communication, 2012). Determining rates of carbonate formation and erosion in subaerial peridotite in a very humid climate, such as New Caledonia, and a moderate climate, such as California, would provide useful comparisons. Another useful comparison would be to determine rates of carbonate formation and erosion in ophiolites of different ages (younger and older than Samail) and degrees of alteration. Presumably, rates of ongoing, low-temperature carbonate formation could vary (slow?) as peridotite becomes increasingly carbonated.

There is also formation of carbonate minerals through alteration of other types of subaerially exposed ultramafic rocks, such as kimberlite (e.g. Exley and Jones, 1983) and 
komatiite (e.g. Lahaye et al., 1995; Barnes et al., 2009), although there are no estimates of natural carbonation rates for these rock types.

There is also potential for significant carbonate formation in seafloor exposures of peridotite. For example, carbonates have been forming for the past $\sim 100,000$ years at Lost City, a peridotite-hosted hydrothermal field located on the Atlantis Massif $\sim 15 \mathrm{~km}$ off-axis along the Mid-Atlantic Ridge (Ludwig et al., 2011). It is also estimated that ultramafic rocks comprise about $50 \%$ of the rocks exposed along slow-spreading ridges (Escartin et al., 2008), so carbonation of these peridotites through low temperature weathering could be an important sink for $\mathrm{CO}_{2}$. However, the total volume of seafloor exposed peridotites is very poorly constrained.

Based on the estimates of ultramafic bedrock exposure discussed above, we assume that ultramafic rocks cover $\sim 1 \%$ of the continental surface, giving a global subaerial exposure of $\sim 1,486,470 \mathrm{~km}^{2}$ of ultramafic bedrock. Extrapolating the $\mathrm{CO}_{2}$ sequestration rate determined in this thesis for Mg-rich carbonate veins in the Samail Ophiolite $\left(\sim 10^{7} \mathrm{~kg} \mathrm{CO}_{2} / \mathrm{yr}\right)$ to the global subaerial ultramafic exposure provides a rough estimate of $\sim 4 \times 10^{9} \mathrm{~kg} \mathrm{CO}_{2} / \mathrm{yr}$ sequestered globally. Since the Samail carbonation rates are likely slow end-members due to Oman's arid climate, this global estimate represents a minimum value. To put this rate estimate into perspective, it is an order of magnitude lower than the $\sim 4.4-8.4 \times 10^{10} \mathrm{~kg} \mathrm{CO}_{2} / \mathrm{yr}$ (Gerlach, 1989) released by ocean ridge volcanism and is significantly lower than the $\sim 3.5 \times 10^{13} \mathrm{~kg}$ of anthropogenic $\mathrm{CO}_{2}$ emissions in 2010 (Friedlingstein et al., 2010). 


\section{References:}

Barnes, S.J., Wells, M.A., and Verrall, M.R. 2009. Effects of magmatic processes, serpentinization, and talc-carbonate alteration on sulfide mineralogy and ore textures in the Black Swan disseminated nickel sulfide deposit, Yilgarn Craton. Economic Geology, Vol. 104, No. 4: 539-562.

Braucher, R., Benedetti, L., Bourlés, D.L., Brown, E.T., and Chardon, D. 2005. Use of in situ-produced ${ }^{10} \mathrm{Be}$ in carbonate-rich environments: A first attempt. Geochimica et Cosmochimica Acta, Vol. 69, No. 6: 1473-1478.

Clark, I.D. and Fontes, J.-C. 1990. Paleoclimate reconstruction in northern Oman based on carbonates from hyperalkaline groundwaters. Quaternary Research, Vol. 33: 320-336.

Clark, I.D., Fontes, J.-C., and Fritz, P. 1992. Stable isotope disequilibria in travertine from high $\mathrm{pH}$ waters: Laboratory investigations and field observations from Oman. Geochimica et Cosmochimica Acta, Vol. 56: 2041-2050.

Cole, J.M., Rasbury, E. T., Hanson, G.N., Montañez, I.P., and Pedone, V.A. 2005. Using $\mathrm{U}-\mathrm{Pb}$ ages of Miocene tufa for correlation in a terrestrial succession, Barstow Formation, California. Geological Society of America Bulletin, Vol. 117, No. 3-4: 276-287.

Coleman, R.G. 1977. Ophiolites. New York: Springer-Verlag, 229 pp.

Cucchi, F., Forti, F., and Furlani S. 2006. Lowering rates of limestone along the western Italian shoreline and the Gulf of Trieste. Geografia Fisica e Dinamica Quaternaria, Vol. 29: 61-69.

Dilek, Y. and Furnes, H. 2011. Ophiolite genesis and global tectonics: Geochemical and tectonic fingerprinting of ancient oceanic lithosphere. Geological Society of America Bulletin, Vol. 123, No. 3-4: 387-411.

Dockhorn, B., Neumaier, S., Hartmann, F.J., Petitjean, C., Faestermann, H., Korschinek, G., Morinaga, H., and Nolte, E. 1991. Determination of erosion rates with cosmic ray produced ${ }^{36} \mathrm{Cl}$. Zeitschrift für Physik A: Hadrons and Nuclei, Vol. 341: 117119.

Eiler, J.M. 2007. "Clumped-isotope" geochemistry - The study of naturally-occurring, multiply-substituted isotopologues. Earth and Planetary Science Letters, Vol. 262: 309-327. 
Eiler, J.M. 2011. Paleoclimate reconstruction using carbonate clumped isotope thermometry. Quaternary Science Reviews, Vol. 30: 3575-3588.

Escartin, J., Smith, D., Cann, J., Schouten, H., Langmuir, C., and Escrig, S. 2008. Central role of detachment faults in accretion of slow-spreading oceanic lithosphere. Nature, Vol. 455: 790-794.

Exley, R.A. and Jones, A.P. $1983 .{ }^{87} \mathrm{Sr}{ }^{86} \mathrm{Sr}$ in kimberlitic carbonates by ion microprobe: Hydrothermal alteration, crustal contamination, and relation to carbonatite. Contributions to Mineralogy and Petrology, Vol. 83, No. 3-4: 288-292.

Friedlingstein, P., Houghton, R.A., Marland, G., Hackler, J., Boden, T.A., Conway, T.J., Canadell, J.G., Raupach, M.R., Ciais, P., and Le Quéré, C. 2010. Update on $\mathrm{CO}_{2}$ emissions. Nature Geoscience, Vol. 3: 811-812.

Gerlach, T.M. 1989. Degassing of carbon dioxide from basaltic magma at spreading centers II. Mid-ocean ridge basalts. Journal of Volcanology and Geothermal Research, Vol. 39: 221-232.

Gislason, S.R. and Oelkers, E. H. 2011. Silicate rock weathering and the global carbon cycle. In: Harmon, R.S. and Parker, A. (eds.) Frontiers in Geochemistry: Contribution of Geochemistry to the Study of the Earth. Oxford: Blackwell Publishing Ltd., 84-103.

Goldstein, R.H. 1986. Reequilibration of fluid inclusions in low-temperature calcium carbonate cement. Geology, Vol. 14: 792-795.

Handwerger, D.A., Cerling, T.E., and Bruhn, R.L. 1999. Cosmogenic ${ }^{14} \mathrm{C}$ in carbonate rocks. Geomorphology, Vol. 27: 13-24.

Havig, J.R., Raymond, J., Meyer-Dombard, D., Zolotova, N., and Shock, E.L. (2011) Merging isotopes and community genomics in a siliceous sinter-depositing hot spring. Journal of Geophysical Research 116, G01005, doi:10.1029/2010JG001415.

High C. and Hanna, G.K. 1970. A method for the direct measurement of erosion of rock surfaces. British Geomorphological Research Group Technical Bulletin, Vol. 5, $24 \mathrm{pp}$.

Jahn, B.-M. and Cuvellier, H. 1994. $\mathrm{Pb}-\mathrm{Pb}$ and $\mathrm{U}-\mathrm{Pb}$ geochronology of carbonate rocks: an assessment. Chemical Geology, Vol. 115: 125-151.

Kelemen, P.B. and Matter, J.M. 2008. In situ carbonation of peridotite for $\mathrm{CO}_{2}$ storage. Proceedings of the National Academy of Sciences, Vol. 105, No.45: 17295-17300. 
Kelemen, P.B., Matter, J.M., Streit, E.E., Rudge, J.F., Curry, W.B., and Blusztajn, J. 2011. Rates and mechanisms of mineral carbonation in peridotite: Natural processes and recipes for enhanced, in situ $\mathrm{CO}_{2}$ capture and storage. Annual Reviews of Earth and Planetary Sciences, Vol. 39: 545-576.

Lackner, K.S., Wendt, C.H., Butt, D.P., Joyce, E.L., and Sharp, D.H. 1995. Carbon dioxide disposal in carbonate minerals. Energy, Vol. 20: 1153-1170.

Lackner, K.S. 2002. Carbonate chemistry for sequestering fossil carbon. Annual Review of Energy and the Environment, Vol. 27: 193-232.

Lahaye, Y., Arndt, N., Byerly, G., Chauvel, C., Fourcade, S., and Gruau, G. 1995. The influence of alteration on the trace-element and $\mathrm{Nd}$ isotopic compositions of komatiites. Chemical Geology, Vol. 126, No. 1: 43-64.

Ludwig, K.A., Shen, C.-C., Kelley, D.S., Cheng, H., and Edwards, L.R. 2011. U-Th systematic and ${ }^{230} \mathrm{Th}$ ages of carbonate chimneys at the Lost City hydrothermal field. Geochmica et Cosmochimica Acta, Vol. 75: 1869-1888.

Matter, J.M. and Kelemen, P.B. 2009. Permanent storage of carbon dioxide in geological reservoirs by mineral carbonation. Nature Geoscience, Vol. 2: 837-841.

Moorbath, S., Taylor, P.N., Orpen, J.L., Treloar, P., and Wilson, J.F. 1987. First direct radiometric dating of Archean stromatolite limestone. Nature, Vol. 326: 865-867.

Neal, C. and Stanger, G. 1985. Past and present serpentinization of ultramafic rocks: An example from the Semail ophiolite nappe of northern Oman. In: Drewer, J.I. (ed.), The Chemistry of Weathering. Holland: D. Reidel Publishing Company: 249-275.

Pentecost, A. 2005. Travertine. Berlin: Springer-Verlag, pp. 445.

Peucker-Ehrenbrink, B. and Miller, M. W. 2002. Quantitative bedrock geology of the conterminous United States of America. Geochemistry, Geophysics, Geosystems, Vol. 3, No. 10: 4 pp.

Peucker-Ehrenbrink, B. and Miller, M.W. 2003. Quantitative bedrock geology of Alaska and Canada. Geochemistry, Geophysics, Geosystems, Vol. 4, No. 4: 10 pp.

Peucker-Ehrenbrink, B. and Miller, M.W. 2004. Quantitative bedrock geology of east and Southeast Asia (Brunei, Cambodia, eastern and southeastern China, east Timor, Indoneasia, Japan, Laos, Malaysia, Myanmar, North Korea, Papua New Guinea, Philippines, far-eastern Russia, Singapore, South Korea, Taiwan, Thailand, Vietnam). Geochemistry, Geophysics, Geosystems, Vol. 5, No. 1: 8 pp. 
Peucker-Ehrenbrink, B. and Miller, M.W. 2007. Quantitative bedrock geology of the continents and large-scale drainage regions. Geochemistry, Geophysics, Geosystems, Vol. 8, No. 6: 10 pp.

Richards, D.A., Bottrell, S. H., Cliff, R.A., Ströhle, K., and Rowe, P.J. 1998. U-Pb dating of a speleothem of Quaternary age. Geochimica et Cosmochimica Acta, Vol. 62, No. 23/24: 3683-3688.

Richter, D.K., Götte, Th., Götze, J., and Neuser, R.D. 2003. Progress in application of cathodoluminescence (CL) in sedimentary petrology. Mineralogy and Petrology, Vol. 79, No. 3-4: 127-166.

Schandl, E.S. and Naldrett, A.J. 1992. $\mathrm{CO}_{2}$ metasomatism of serpentinites, south of Timmins, Ontario. Canadian Mineralogist, Vol. 30: 93-108.

Seifritz, W. 1990. $\mathrm{CO}_{2}$ disposal by means of silicates. Nature, Vol. 345: 486.

Smith, P.E. and Farquhar, R.M. 1989. Direct dating of Phanerozoic sediments by the ${ }^{238} \mathrm{U}_{-}{ }^{206} \mathrm{~Pb}$ method. Nature, Vol. 341: 518-521.

Spate, A.P., Jennings, J.N., Smith, D.I., and Greenaway, M.A. 1985. The micro-erosion meter: use and limitations. Earth Surface Processes and Landforms, Vol. 10: 427440.

Streit, E.E., Kelemen, P.B., and Eiler, J.M. Accepted. Coexisting serpentine and quartz from carbonate-bearing serpentinized peridotite. Contributions to Mineralogy and Petrology.

Stone, J.O., Allan, G.L., Fifield, L.K., and Cresswell, R.G. 1996. Cosmogenic chlorine36 from calcium spallation. Geochimica et Cosmochimica Acta, Vol. 60, No. 4: 679-692.

Stone, J. O. H., Evans, J. M., Fifield, L. K., and Allan, G. L., and Cresswell, R. G. 1998. Cosmogenic chlorine-36 production in calcite by muons. Geochimica et Cosmochimica Acta, Vol. 62, No. 3: 433-454.

ten Have, T. and Heijnen, W. 1985. Cathodoluminescence activation and zonation in carbonate rocks: an experimental approach. Geologie en Mijnbouw, Vol. 64: 297310.

Wilson, S.A., Barker, S.L.L., Dipple, G.M., and Atudorei, V. 2010. Isotopic disequilibrium during uptake of atmospheric $\mathrm{CO}_{2}$ into mine process waters: 
Implications for $\mathrm{CO}_{2}$ sequestration. Environmental Science and Technology, Vol. 44, No. 24: 9522-9529. 


\section{Appendix A: Carbonate Dissolution Experiments for $U$ and Th Analyses}

\section{A.1 Motivation:}

Carbonate dissolution experiments were conducted to investigate whether carbonate material could be chemically separated from silicate detritus without causing fractionation of $\mathrm{U}$ and Th. Previous studies (e.g. Ku and Liang, 1984; Bischoff and Fitzpatrick, 1991; Luo and $\mathrm{Ku}, 1991$; refer to reference list in Chapter 3) had suggested that fractionation of $\mathrm{U}$ and $\mathrm{Th}$ concentrations and isotopes occurs during partial dissolution of carbonate-silicate detritus mixtures, bringing into question whether partial dissolutions could be employed for $\mathrm{U}$ and Th analysis of Samail carbonates. Sample locations and descriptions are presented in Table 1, $\mathrm{U}$ and Th concentrations are presented in Table 2, and $\mathrm{U}$ and Th isotopes are presented in Table 3.

\section{A.2 Methods:}

\section{A.2.1 Travertine Dissolution Experiments:}

Dissolution experiments were carried out on Samail surface travertines and one travertine vein (OM09-84C-MS). Three sets of experiments were carried out, and $U$ and Th concentrations and isotopes were measured in all dissolution experiment fractions.

The first experiment was designed to constrain the amount and approximate composition of silicate residue present in the travertines. Two similar fractions (same amount of material weighed out, same grain size, same amount of hand-picking to remove large silicate fragments) of the same travertine sample were dissolved in two different ways. In the first dissolution, the travertine was completely dissolved using 
$\mathrm{HNO}_{3}, \mathrm{HF}, \mathrm{HClO}_{4}$, and $\mathrm{H}_{3} \mathrm{BO}_{3}$. In the second dissolution, the majority of the carbonate material was dissolved using a weak $(0.5-1 \mathrm{~N}) \mathrm{HNO}_{3}$ leach and then the remaining material, which was dominantly silicate residue, was dried down and weighed. This silicate detritus residue was then dissolved separately, employing $\mathrm{HNO}_{3}, \mathrm{HF}, \mathrm{HClO}_{4}$, and $\mathrm{H}_{3} \mathrm{BO}_{3}$ to bring all material into solution. For the surface travertines, the silicate residues were significant, approximately $5-10 \%$ of the total weight of the sample. However, for the travertine vein the silicate residue was negligible $(<1 \%$ by weight) and was not analyzed due to its small size.

In the second experiment, multiple dissolutions were carried out for travertine terrace sample OM09-107C-MS to investigate if: (1.) spiking protocols affected $U$ and Th fractionation and (2.) if grain size affected $U$ and Th fractionation. For this sample, three sets of paired weak acid and silicate residue dissolutions were obtained. These are indicated by numbers. For instance, OM09-107C-MS-Weak Acid \#1 and OM09-107CMS-Silicate Residue \#1 are the weak acid and silicate residue fractions from the first paired set of dissolutions. For the first paired dissolution (\#1), the $0.85-2 \mathrm{~mm}$ size fraction was dissolved and spiked prior to dissolution (the spike was added to MilliQ water on top of undissolved carbonate grains) to investigate if any fractionation occurred during dissolution. The second paired dissolution $(\# 2)$ is the same as the first dissolution except that the sample was spiked after dissolution, which is the normal protocol. The third paired dissolution (\#3) was also spiked after dissolution, but the smaller 0.6 to 0.85 mm size fraction was dissolved. The total dissolution for OM09-107C-MS was spiked after dissolution, and the larger 0.85-2 $\mathrm{mm}$ size fraction was dissolved. 
Finally, in the third experiment, a progressive dissolution was carried out for travertine terrace sample OM09-107C-MS in order to further constrain the progression of $\mathrm{U}$ and $\mathrm{Th}$ fractionation during dissolution. A total of 5.8830 grams of hand-picked travertine chips was weighed into a large $(120 \mathrm{~mL})$ Teflon beaker. The sample was first dissolved with weak $(0.5-1 \mathrm{~N}) \mathrm{HNO}_{3}$, then with stronger $\mathrm{HNO}_{3}(7 \mathrm{~N}$ then concentrated), and finally with concentrated $\mathrm{HNO}_{3}$, $\mathrm{HF}, \mathrm{HCLO}_{4}$, and $\mathrm{H}_{3} \mathrm{BO}_{3}$. Five progressive fractions (labeled DE 1-5) were analyzed for U-Th concentrations and isotopes. The progressive fractions had weights (in order) of 0.7633 grams, 0.8245 grams, 1.4865 grams, 1.1350 grams, and 1.2400 grams.

\section{A.2.2 Mg-Rich Vein Dissolution Experiments:}

This final dissolution experiment was designed to constrain the amount and approximate composition of silicate residue present in the Mg-rich veins. Weak $(0.5-1 \mathrm{~N})$ $\mathrm{HNO}_{3}$ leaches and total dissolutions were carried out on two similar fractions (same amount of material weighed out, same grain size, same amount of hand-picking to remove large silicate fragments) for many of the samples. A few of the veins have only a weak acid or total dissolution (rather than a paired set of dissolutions). The silicate residues for the Mg-rich veins were negligible ( $<1 \%$ by weight) and were not analyzed due to their small sizes. Because very large samples sizes (30-40 grams, see Chapter 3) are required for isotopic analysis of the low concentration Mg-rich veins, only $U$ and $\mathrm{Th}$ concentrations were measured in the dissolution experiment fractions. 


\section{A.3 Results:}

\section{A.3.1 Travertine Dissolution Experiment \#1:}

The surface travertines (Figure A1) exhibit a noticeable difference in $\mathrm{U}$ and $\mathrm{Th}$ concentrations for different types of dissolutions performed on the same sample. Relative to the total dissolutions, the weak acid dissolutions have lower $\mathrm{Th}$ concentrations and variable U concentrations that can be slightly lower (e.g. sample OM09-106C-MS-A), about the same (e.g. sample OM09-107C-MS), or slightly higher (e.g. sample OM0910COPS-MS). The silicate residue dissolutions have highly elevated Th concentrations and similar to slightly elevated $U$ concentrations relative to the weak acid dissolutions and total dissolutions. In contrast, there are no significant differences in $\mathrm{U}$ and $\mathrm{Th}$ concentration for the weak acid and total dissolutions conducted on travertine vein OM09-84C-MS. This vein formed in the subsurface and likely incorporated less silicate detritus than the surface travertine deposits.

The surface travertines exhibit noticeable differences in $\mathrm{U}$ and $\mathrm{Th}$ isotopic composition for different types of dissolutions performed on the same sample. On a plot of $\left({ }^{230} \mathrm{Th} /{ }^{232} \mathrm{Th}\right)$ vs. $\left({ }^{238} \mathrm{U} /{ }^{232} \mathrm{Th}\right)$ (Figure A2), the weak acid dissolutions plot to the right of the equiline and thus have ${ }^{238} \mathrm{U}$ excesses. The silicate residue dissolutions plot to the left of the equiline and thus have ${ }^{230} \mathrm{Th}$ excesses. The total dissolutions plot either on or to the right of the equiline. For individual samples, the total dissolutions plot closer to the equiline than the corresponding weak acid dissolutions. The three sets of weak acid, silicate residue, and total dissolutions on sample OM09-107C-MS indicate that neither 
grain size nor the timing of spiking (before or after dissolution) significantly affect $U$ and Th isotopes.

Figures A3 and A4 illustrate that while weak acid, silicate residue, and total dissolutions for travertines cover similar $U$ concentration ranges, the silicate residues generally have lower $\left({ }^{234} \mathrm{U} /{ }^{238} \mathrm{U}\right)$, which is closer to equilibrium, and lower $\left({ }^{230} \mathrm{Th} /{ }^{232} \mathrm{Th}\right)$, which indicates the presence of significant detrital ${ }^{232} \mathrm{Th}$. However, there is no systematic relationship between $U$ and $T h$ concentrations and isotopes, which suggests that concentrations alone cannot be used to reliably identify the presence of aluminosilicate detritus. Total dissolutions with corrections for detrital Th (and perhaps also U) inputs must be used for ${ }^{230} \mathrm{Th}$ dating of surface travertines.

\section{A.3.2 Travertine Dissolution Experiment \#2:}

The three sets of weak acid, silicate reisdue, and total dissolutions on travertine terrace sample OM09-107C-MS indicate the timing of spiking (before or after dissolution) nor the grain size significantly affect $U$ and Th concentrations. All of the silicate residue dissolutions display highly elevated Th concentrations and slightly elevated $U$ concentrations relative to the corresponding weak acid dissolutions and total dissolutions.

\section{A.3.3 Travertine Dissolution Experiment \#3:}

The progressive dissolution experiment has low and fairly constant $\mathrm{U}$ and $\mathrm{Th}$ concentrations for the first four acid leaches. The fifth acid leach has significantly higher $\mathrm{U}$ and Th concentrations. For the dissolution experiments on travertine sample OM09107C-MS (Figures A2, A3, and A4), there is a significant difference in the $\mathrm{U}$ and Th 
isotopic composition of the acid leaches even though there is very little difference in $U$ and Th concentration for the first four acid leaches (Figure A1). The first four acid leaches have ${ }^{238} \mathrm{U}$ excesses while the fifth acid leach has a significant ${ }^{230} \mathrm{Th}$ excess. Generally, the early leaches have larger ${ }^{238} \mathrm{U}$ excesses than the later leaches. However, dissolution fraction \#4 has more of a ${ }^{238} \mathrm{U}$ excess than fraction \#3, which indicates that the behavior is not completely systematic. The behavior of $U$ and $T h$ isotopes in the progressive dissolution experiment indicates that the isotopic composition of various acid leaches of surface travertines can be quite variable depending on the amount of material dissolved and the strength of the dissolving acid.

\section{A.3.4 Mg-Rich Vein Dissolution Experiments}

The results of $U$ and Th measurements for dissolution experiments conducted on Mg-rich carbonate veins are shown in Figures A5 and A6. Since some Mg-rich carbonate veins only have a weak acid dissolution or a total dissolution (rather than a paired set of dissolutions), the results are first broken down in a broad fashion as "weak acid" or "total dissolution" in Figure A5. This figure illustrates that weak acid and total dissolutions of Mg-rich carbonate veins cover a similar range of $U$ and Th concentrations. Paired weak acid and total dissolutions for $\mathrm{Mg}$-rich carbonate veins illustrate that $\mathrm{U}$ and $\mathrm{Th}$ concentrations are similar for both types of dissolutions on individual samples (Figure A6).

\section{A.4 Summary:}

The progressive dissolution experiments on surface travertines indicate that $U$ and Th concentrations and isotopes can vary significantly between weak acid, silicate residue, 
and total dissolutions for these samples, which contain significant ( $5-10 \%$ by weight) admixed aluminosilicate detritus. There is likely significant fractionation of $U$ and $T h$ concentrations and isotopes occurring during partial dissolutions. Therefore, total dissolutions with corrections for detrital Th (and also possibly for $U$ ) must be employed dating these travertines using the ${ }^{230} \mathrm{Th}$ technique. Travertine vein OM09-84C-MS and Mg-rich carbonate veins contain significantly less aluminosilicate detritus $(<1 \%$ by weight) than the surface travertines. Progressive dissolution experiments for these samples do not find significant differences in $U$ and $T h$ concentrations. Therefore, it is less important to employ total dissolutions when dating subsurface carbonate veins using the ${ }^{230}$ Th dating technique. Nevertheless, in this study (see Chapter 3) total dissolutions were employed for all carbonates evaluated for ${ }^{230} \mathrm{Th}$ dating. 
Tables:

Table 1: Sample Locations and Descriptions

\begin{tabular}{|c|c|c|c|c|c|c|}
\hline Sample Name: & Location: & $\begin{array}{l}\text { UTM- } \\
\text { Easting: }\end{array}$ & $\begin{array}{c}\text { UTM- } \\
\text { Northing: }\end{array}$ & Description: & $\begin{array}{l}\text { Major Minerals } \\
\text { (XRD): }\end{array}$ & $\begin{array}{c}\text { Trace Minerals } \\
\text { (XRD): }\end{array}$ \\
\hline \multicolumn{7}{|l|}{ Travertines: } \\
\hline $\begin{array}{l}\text { OM09-106C-MS-A } \\
\text { (Top) }\end{array}$ & $\begin{array}{l}\text { Wadi Uqaybah } \\
\text { Travertine }\end{array}$ & 0426245 & 2633924 & Travertine terrace & calcite & none \\
\hline $\begin{array}{l}\text { OM09-106C-MS-B } \\
\text { (Bottom) }\end{array}$ & $\begin{array}{l}\text { Wadi Uqaybah } \\
\text { Travertine }\end{array}$ & 0426245 & 2633924 & Travertine terrace & calcite & unidentified clay \\
\hline OM09-107C-MS & $\begin{array}{l}\text { Wadi Uqaybah } \\
\text { Travertine }\end{array}$ & 0426309 & 2633950 & Travertine terrace & calcite & unidentified clay \\
\hline OM09-109C-MS & $\begin{array}{l}\text { Wadi Uqaybah } \\
\text { Travertine }\end{array}$ & 0426208 & 2633925 & $\begin{array}{c}\text { Channel-filling } \\
\text { travertine }\end{array}$ & calcite & none \\
\hline OM09-10COPS-MS & $\begin{array}{l}\text { Wadi Uqaybah } \\
\text { Travertine }\end{array}$ & 0426183 & 2633965 & $\begin{array}{l}\text { Recently-formed } \\
\text { travertine precipitate }\end{array}$ & calcite & quartz, unidentified clay \\
\hline OM09-84C-MS & $\begin{array}{c}\text { Wadi Sudari } \\
\text { Travertine }\end{array}$ & 0443082 & 2650304 & Travertine vein & calcite, brucite & $\begin{array}{l}\text { hydromagnesite?, } \\
\text { unidentified clay }\end{array}$ \\
\hline \multicolumn{7}{|l|}{$\begin{array}{l}\text { Mg-Rich Carbonate } \\
\text { Outcrop Veins: }\end{array}$} \\
\hline OM09-47C-MS & Fanja Roadcut & 0609304 & 2597565 & $\begin{array}{c}\text { Carbonate outcrop } \\
\text { vein }\end{array}$ & magnesite & unidentified clay \\
\hline OM10-13C-MG & Fanja Roadcut & 0609451 & 2597416 & $\begin{array}{l}\text { Carbonate outcrop } \\
\text { vein }\end{array}$ & magnesite & dolomite, calcite \\
\hline OM10-53C-MG & $\begin{array}{l}\text { Al-Wuqbah } \\
\text { Roadcut }\end{array}$ & 0440396 & 2643047 & $\begin{array}{l}\text { Carbonate outcrop } \\
\text { vein }\end{array}$ & magnesite & none \\
\hline OM09-91C-MS & $\begin{array}{l}\text { Wadi Sudari } \\
\text { Campsite }\end{array}$ & 0446151 & 2647471 & $\begin{array}{l}\text { Carbonate outcrop } \\
\text { vein } \\
\end{array}$ & magnesite & calcite \\
\hline OM10-82C-MG & $\begin{array}{l}\text { Wadi Sudari } \\
\text { Campsite }\end{array}$ & 0445905 & 2647602 & $\begin{array}{c}\text { Carbonate outcrop } \\
\text { vein } \\
\end{array}$ & magnesite & $\begin{array}{l}\text { dolomite, calcite, } \\
\text { unidentified clay }\end{array}$ \\
\hline OM10-83C-MG & $\begin{array}{l}\text { Wadi Sudari } \\
\text { Campsite }\end{array}$ & 0445905 & 2647602 & $\begin{array}{l}\text { Carbonate outcrop } \\
\text { vein } \\
\end{array}$ & dolomite, calcite & $\begin{array}{l}\text { magnesite, unidentified } \\
\text { clay }\end{array}$ \\
\hline
\end{tabular}




\begin{tabular}{|c|c|c|c|c|c|c|}
\hline Sample Name: & Location: & $\begin{array}{c}\text { UTM- } \\
\text { Easting: }\end{array}$ & $\begin{array}{c}\text { UTM- } \\
\text { Northing: }\end{array}$ & Description: & $\begin{array}{l}\text { Major Minerals } \\
\text { (XRD): }\end{array}$ & $\begin{array}{c}\text { Trace Minerals } \\
\text { (XRD): }\end{array}$ \\
\hline OM10-84C-MG & $\begin{array}{l}\text { Wadi Sudari } \\
\text { Campsite }\end{array}$ & 0445991 & 2647684 & $\begin{array}{c}\text { Carbonate outcrop } \\
\text { vein }\end{array}$ & brucite, calcite & $\begin{array}{c}\text { magnesite, dolomite, } \\
\text { unidentified clay }\end{array}$ \\
\hline \multicolumn{7}{|l|}{$\begin{array}{l}\text { Mg-Rich Carbonate } \\
\text { Roadcut Veins: }\end{array}$} \\
\hline OM09-35C-MS & $\begin{array}{l}\text { Qafeefah } \\
\text { Roadcut }\end{array}$ & 0647852 & 2537682 & $\begin{array}{c}\text { Carbonate/serpentine } \\
\text { roadcut vein }\end{array}$ & magnesite & $\begin{array}{l}\text { chrysotile, unidentified } \\
\text { clay }\end{array}$ \\
\hline OM09-36C-MS & $\begin{array}{l}\text { Qafeefah } \\
\text { Roadcut }\end{array}$ & 0647818 & 2537657 & $\begin{array}{c}\text { Carbonate roadcut } \\
\text { vein }\end{array}$ & magnesite & calcite, dolomite \\
\hline OM09-38C-MS & $\begin{array}{l}\text { Qafeefah } \\
\text { Roadcut }\end{array}$ & 0647782 & 2537641 & $\begin{array}{c}\text { Thin carbonate on } \\
\text { roadcut surface }\end{array}$ & magnesite & $\begin{array}{l}\text { dolomite, unidentified } \\
\text { clay }\end{array}$ \\
\hline OM10-26C-MG & $\begin{array}{l}\text { Qafeefah } \\
\text { Roadcut }\end{array}$ & 0647791 & 2537644 & $\begin{array}{c}\text { Carbonate/serpentine } \\
\text { roadcut vein }\end{array}$ & magnesite & kaolinite, dolomite \\
\hline OM10-27C-MG & $\begin{array}{l}\text { Qafeefah } \\
\text { Roadcut }\end{array}$ & 0647791 & 2537644 & $\begin{array}{c}\text { Carbonate/serpentine } \\
\text { roadcut vein }\end{array}$ & magnesite & kaolinite, dolomite \\
\hline OM09-55C-MS & Fanja Roadcut & 0609351 & 2597507 & $\begin{array}{l}\text { Carbonate/serpentine } \\
\text { roadcut vein }\end{array}$ & dolomite & $\begin{array}{c}\text { aragonite?, unidentified } \\
\text { clay }\end{array}$ \\
\hline OM09-57C-MS & Fanja Roadcut & 0609356 & 2597501 & $\begin{array}{l}\text { Carbonate/serpentine } \\
\text { roadcut vein }\end{array}$ & dolomite & $\begin{array}{c}\text { aragonite?, calcite, } \\
\text { unidentified clay }\end{array}$ \\
\hline OM09-58C-MS & Fanja Roadcut & 0609370 & 2597506 & $\begin{array}{c}\text { Carbonate/serpentine } \\
\text { roadcut vein }\end{array}$ & dolomite, periclase? & $\begin{array}{l}\text { aragonite?, unidentified } \\
\text { clay }\end{array}$ \\
\hline OM09-63C-MS & Fanja Roadcut & 0609470 & 2597481 & $\begin{array}{c}\text { Carbonate roadcut } \\
\text { vein }\end{array}$ & magnesite & unidentified clay \\
\hline OM10-48C-MG & $\begin{array}{c}\text { Al-Wuqbah } \\
\text { Roadcut }\end{array}$ & 0440574 & 2643360 & $\begin{array}{c}\text { Carbonate/serpentine } \\
\text { roadcut vein }\end{array}$ & dolomite & $\begin{array}{l}\text { chrysotile, kaonlinite, } \\
\text { other unidentified clay }\end{array}$ \\
\hline OM10-52C-MG & $\begin{array}{l}\text { Al-Wuqbah } \\
\text { Roadcut }\end{array}$ & 0440396 & 2643047 & $\begin{array}{c}\text { Carbonate roadcut } \\
\text { vein }\end{array}$ & magnesite & $\begin{array}{l}\text { halite?, unidentified } \\
\text { clay }\end{array}$ \\
\hline OM10-53C-MG & $\begin{array}{l}\text { Al-Wuqbah } \\
\text { Roadcut }\end{array}$ & 0440396 & 2643047 & $\begin{array}{c}\text { Thin carbonate on } \\
\text { roadcut surface }\end{array}$ & magnesite & none \\
\hline OM10-54C-MG & $\begin{array}{l}\text { Al-Wuqbah } \\
\text { Roadcut }\end{array}$ & 0440832 & 2643931 & $\begin{array}{l}\text { Carbonate/serpentine } \\
\text { roadcut vein }\end{array}$ & dolomite & $\begin{array}{l}\text { smectitie-kaolinite, } \\
\text { other unidentified clay }\end{array}$ \\
\hline
\end{tabular}

The UTM coordinates were measured in the WGS84 zone. Major and trace mineral identifications were determined on powdered samples by X-Ray diffraction at Woods Hole Oceanographic Institution. The minerals were identified using MacDiff, Version 4.2. 
Table 2: $U$ and Th Concentrations

\begin{tabular}{|c|c|c|c|c|c|}
\hline Sample Name: & $\mathrm{U}(\mathrm{ppm}):$ & $2 \sigma(\%):$ & Th (ppm): & $2 \sigma(\%):$ & Th/U: \\
\hline \multicolumn{6}{|l|}{ Travertines: } \\
\hline OM09-106C-MS-A-Weak Acid & 0.9458 & 1.40 & 0.1839 & 1.20 & 0.1945 \\
\hline OM09-106C-MS-A-Silicate Residue & 0.4797 & 1.42 & 1.5140 & 1.20 & 3.1558 \\
\hline OM09-106C-MS-A-Total & 0.8275 & 1.40 & 0.2917 & 1.20 & 0.3525 \\
\hline OM09-106C-MS-B-Weak Acid & 0.3421 & 1.40 & 0.0870 & 1.20 & 0.2543 \\
\hline OM09-106C-MS-B-Silicate Residue & 0.3149 & 1.40 & 0.9292 & 1.20 & 2.9510 \\
\hline \multirow[t]{3}{*}{ OM09-106C-MS-B-Total } & 0.3434 & 1.40 & 0.1507 & 1.20 & 0.4389 \\
\hline & 0.3456 & 1.40 & 0.1507 & 1.20 & 0.4361 \\
\hline & 0.3445 & 1.40 & 0.1507 & 1.20 & 0.4375 \\
\hline OM09-107C-MS-Weak Acid \#1 & 0.6032 & 1.40 & 0.2220 & 1.21 & 0.3680 \\
\hline OM09-107C-MS-Weak Acid \#2 & 0.5661 & 1.40 & 0.1878 & 1.20 & 0.3317 \\
\hline OM09-107C-MS-Weak Acid \#3 & 0.5557 & 1.40 & 0.2660 & 1.20 & 0.4786 \\
\hline OM09-107C-MS-Silicate Residue \#1 & 0.812 & 1.40 & 2.642 & 1.20 & 3.2547 \\
\hline OM09-107C-MS-Silicate Residue \#2 & 1.279 & 1.40 & 4.744 & 1.21 & 3.7085 \\
\hline OM09-107C-MS-Silicate Residue \#3 & 1.369 & 1.40 & 5.237 & 1.20 & 3.8258 \\
\hline OM09-107C-MS-Total & 0.6016 & 1.40 & 0.4250 & 1.20 & 0.7065 \\
\hline OM09-107C-MS-DE \#1 & 0.2044 & 1.40 & 0.0215 & 1.20 & 0.1054 \\
\hline OM09-107C-MS-DE \#2 & 0.1595 & 1.40 & 0.0282 & 1.20 & 0.1766 \\
\hline OM09-107C-MS-DE \#3 & 0.1658 & 1.40 & 0.0479 & 1.20 & 0.2887 \\
\hline OM09-107C-MS-DE \#4 & 0.1956 & 1.40 & 0.0405 & 1.20 & 0.2073 \\
\hline OM09-107C-MS-DE \#5 & 0.4390 & 1.40 & 0.6012 & 1.20 & 1.3695 \\
\hline OM09-109C-MS-Weak Acid & 0.0332 & 1.40 & 0.0365 & 1.20 & 1.0980 \\
\hline OM09-109C-MS-Silicate Residue & 0.0525 & 1.40 & 0.2372 & 1.20 & 4.5199 \\
\hline OM09-109C-MS-Total & 0.0687 & 1.40 & 0.1072 & 1.20 & 1.5607 \\
\hline OM09-10COPS-MS-Weak Acid & 0.1327 & 1.40 & 0.1102 & 1.20 & 0.8306 \\
\hline \multirow[t]{3}{*}{ OM09-10COPS-MS-Silicate Residue } & 0.4964 & 1.40 & 2.0693 & 1.20 & 4.1686 \\
\hline & 0.5113 & 1.40 & 2.2852 & 1.20 & 4.4694 \\
\hline & 0.5039 & 1.40 & 2.1773 & 1.20 & 4.3190 \\
\hline \multirow[t]{3}{*}{ OM09-10COPS-MS-Total } & 0.2693 & 1.40 & 0.3455 & 1.20 & 1.2829 \\
\hline & 0.1732 & 1.40 & 0.3560 & 1.20 & 2.0551 \\
\hline & 0.2213 & 1.40 & 0.3507 & 1.20 & 1.6690 \\
\hline OM09-84C-MS-Weak Acid & 0.0134 & 1.40 & 0.0041 & 1.20 & 0.3101 \\
\hline OM09-84C-MS-Total & 0.0161 & 1.40 & 0.0041 & 1.20 & 0.2532 \\
\hline \multicolumn{6}{|l|}{ Mg-Rich Carbonate Outcrop Veins: } \\
\hline OM09-47C-MS-Weak Acid & 0.0035 & 1.40 & 0.0009 & 1.20 & 0.2553 \\
\hline \multirow[t]{3}{*}{ OM09-47C-MS-Total } & 0.0030 & 1.40 & 0.0007 & 1.20 & 0.2317 \\
\hline & 0.0007 & 1.40 & 0.0003 & 1.20 & 0.3547 \\
\hline & 0.0019 & 1.40 & 0.0005 & 1.20 & 0.2932 \\
\hline OM10-13C-MG-Weak Acid & 0.0008 & 1.40 & 0.0001 & 1.20 & 0.1865 \\
\hline OM10-13C-MG-Total & 0.0010 & 1.40 & 0.0002 & 1.20 & 0.1683 \\
\hline OM10-53C-MG-Total & 0.0032 & 1.40 & 0.0006 & 1.20 & 0.1819 \\
\hline OM09-91C-MS-Total & 0.0114 & 1.40 & 0.0030 & 1.21 & 0.2626 \\
\hline
\end{tabular}




\begin{tabular}{|c|c|c|c|c|c|}
\hline Sample Name: & $\mathbf{U}(\mathbf{p p m}):$ & $2 \sigma(\%):$ & Th (ppm): & $2 \sigma(\%):$ & Th/U: \\
\hline OM10-82C-MG-Weak Acid & 0.0008 & 1.40 & 0.0003 & 1.20 & 0.4075 \\
\hline OM10-82C-MG-Total & 0.0009 & 1.40 & 0.0003 & 1.20 & 0.2903 \\
\hline OM10-83C-MG-Weak Acid & 0.0066 & 1.40 & 0.0013 & 1.20 & 0.2013 \\
\hline OM10-84C-MG-Weak Acid & 0.0007 & 1.40 & 0.0009 & 1.20 & 1.1810 \\
\hline OM10-84C-MG-Total & 0.0010 & 1.40 & 0.0021 & 1.20 & 1.9955 \\
\hline \multicolumn{6}{|l|}{ Mg-Rich Carbonate Roadcut Veins: } \\
\hline OM09-35C-MS-Weak Acid & 0.0001 & 1.40 & 0.0004 & 1.20 & 3.0006 \\
\hline OM09-35C-MS-Total & 0.0001 & 1.40 & 0.0003 & 1.20 & 3.0990 \\
\hline OM09-36C-MS-Weak Acid & 0.0001 & 1.40 & 0.0002 & 1.20 & 1.3627 \\
\hline OM09-36C-MS-Total & 0.0001 & 1.40 & 0.0003 & 1.20 & 2.9332 \\
\hline \multirow[t]{3}{*}{ OM09-38C-MS-Weak Acid } & 0.0012 & 1.40 & 0.0042 & 1.20 & 3.3492 \\
\hline & 0.0013 & 1.40 & 0.0040 & 1.20 & 3.0050 \\
\hline & 0.0013 & 1.40 & 0.0041 & 1.20 & 3.1771 \\
\hline OM10-26C-MG-Weak Acid & 0.0012 & 1.40 & 0.0034 & 1.20 & 2.8364 \\
\hline \multirow[t]{3}{*}{ OM10-27C-MG-Weak Acid } & 0.0002 & 1.40 & 0.0003 & 1.20 & 1.6366 \\
\hline & 0.0002 & 1.40 & 0.0004 & 1.20 & 2.2994 \\
\hline & 0.0002 & 1.40 & 0.0003 & 1.20 & 1.9680 \\
\hline OM09-55C-MS-Total & 0.0008 & 1.40 & 0.0004 & 1.20 & 0.4777 \\
\hline OM09-57C-MS-Total & 0.0053 & 1.40 & 0.0005 & 1.20 & 0.0978 \\
\hline OM09-58C-MS-Total & 0.0148 & 1.40 & 0.0002 & 1.20 & 0.0111 \\
\hline OM09-63C-MS-Weak Acid & 0.00002 & 1.40 & 0.00003 & 1.20 & 1.8188 \\
\hline \multirow[t]{3}{*}{ OM09-63C-MS-Total } & 0.00003 & 1.40 & 0.00006 & 1.20 & 2.1948 \\
\hline & 0.00004 & 1.40 & 0.00008 & 1.20 & 1.8916 \\
\hline & 0.00003 & 1.40 & 0.00007 & 1.20 & 2.043 \\
\hline OM10-48C-MG-Weak Acid & 0.0006 & 1.40 & 0.0025 & 1.20 & 4.094 \\
\hline OM10-52C-MG-Total & 0.0003 & 1.40 & 0.0017 & 1.20 & 5.609 \\
\hline OM10-53C-MG-Total & 0.0032 & 1.40 & 0.0006 & 1.20 & 0.182 \\
\hline OM10-54C-MG-Weak Acid & 0.0002 & 1.40 & 0.0004 & 1.20 & 2.842 \\
\hline
\end{tabular}

Replicate analyses are indicated in italics, with the average of replicate analyses given in bold. Numbers in red are below the detection limit. 
Table 3: $\mathrm{U}$ and $\mathrm{Th}$ Isotopes for Travertines

\begin{tabular}{|c|c|c|c|c|c|c|c|c|}
\hline Sample Name: & $\begin{array}{l}{ }^{234} \mathrm{U} /{ }^{238} \mathrm{U} \\
(\mathrm{ppm}):\end{array}$ & $2 \sigma(\%):$ & $\begin{array}{c}{ }^{230} \mathrm{Th} /{ }^{232} \mathrm{Th} \\
\text { (ppm): }\end{array}$ & $2 \sigma(\%):$ & $\left({ }^{234} U /{ }^{238} U\right):$ & $\left({ }^{230} \mathrm{Th} /{ }^{232} \mathrm{Th}\right):$ & $\left({ }^{238} \mathrm{U} /{ }^{232} \mathrm{Th}\right):$ & $\left({ }^{230} \mathrm{Th} /{ }^{238} \mathrm{U}\right):$ \\
\hline \multicolumn{9}{|l|}{ Travertines: } \\
\hline $\begin{array}{l}\text { OM09-106C-MS-A- } \\
\text { Weak Acid }\end{array}$ & 64.000 & 0.028 & 20.856 & 0.107 & 1.166 & 3.860 & 15.599 & 0.247 \\
\hline $\begin{array}{l}\text { OM09-106C-MS-A- } \\
\text { Silicate Residue }\end{array}$ & 58.002 & 0.052 & 4.859 & 0.157 & 1.057 & 0.899 & 0.961 & 0.935 \\
\hline $\begin{array}{l}\text { OM09-106C-MS-A- } \\
\text { Total }\end{array}$ & 63.466 & 0.024 & 20.330 & 0.098 & 1.156 & 3.763 & 8.606 & 0.437 \\
\hline $\begin{array}{c}\text { OM09-106C-MS-B- } \\
\text { Weak Acid }\end{array}$ & 63.290 & 0.036 & 20.356 & 0.134 & 1.153 & 3.768 & 11.930 & 0.316 \\
\hline $\begin{array}{l}\text { OM09-106C-MS-B- } \\
\text { Silicate Residue }\end{array}$ & 59.821 & 0.059 & 20.924 & 0.107 & 1.090 & 3.873 & 1.028 & 3.767 \\
\hline $\begin{array}{l}\text { OM09-106C-MS-B- } \\
\text { Total }\end{array}$ & 63.427 & 0.038 & 14.707 & 0.171 & 1.156 & 2.722 & 6.912 & 0.394 \\
\hline & 63.390 & 0.039 & 14.693 & 0.090 & 1.155 & 2.719 & 6.956 & 0.391 \\
\hline & 63.409 & 0.039 & 14.700 & 0.131 & 1.155 & 2.721 & 6.934 & 0.392 \\
\hline $\begin{array}{c}\text { OM09-107C-MS-Weak } \\
\text { Acid \#1 }\end{array}$ & 71.458 & 0.032 & 42.325 & 0.058 & 1.302 & 7.834 & 8.243 & 0.950 \\
\hline $\begin{array}{c}\text { OM09-107C-MS-Weak } \\
\text { Acid \#2 }\end{array}$ & 70.256 & 0.023 & 41.507 & 0.082 & 1.280 & 7.682 & 9.147 & 0.840 \\
\hline $\begin{array}{l}\text { OM09-107C-MS-Weak } \\
\text { Acid \#3 }\end{array}$ & 69.814 & 0.032 & 33.021 & 0.077 & 1.272 & 6.112 & 6.338 & 0.964 \\
\hline $\begin{array}{c}\text { OM09-107C-MS-Silicate } \\
\text { Residue \#1 }\end{array}$ & 60.305 & 0.031 & 9.035 & 0.070 & 1.099 & 1.672 & 0.932 & 1.794 \\
\hline $\begin{array}{c}\text { OM09-107C-MS-Silicate } \\
\text { Residue \#2 }\end{array}$ & 59.897 & 0.036 & 11.678 & 0.063 & 1.091 & 2.161 & 0.818 & 2.642 \\
\hline $\begin{array}{c}\text { OM09-107C-MS-Silicate } \\
\text { Residue \#3 }\end{array}$ & 61.140 & 0.052 & 11.071 & 0.071 & 1.114 & 2.049 & 0.793 & 2.584 \\
\hline OM09-107C-MS-Total & 68.860 & 0.032 & 23.603 & 0.082 & 1.255 & 4.369 & 4.294 & 1.017 \\
\hline OM09-107C-MS-DE \#1 & 64.855 & 0.038 & 27.515 & 0.186 & 1.182 & 5.093 & 28.771 & 0.177 \\
\hline OM09-107C-MS-DE \#2 & 64.915 & 0.041 & 26.341 & 0.200 & 1.183 & 4.875 & 17.179 & 0.284 \\
\hline OM09-107C-MS-DE \#3 & 65.300 & 0.049 & 33.674 & 0.077 & 1.190 & 6.233 & 10.508 & 0.593 \\
\hline
\end{tabular}




\begin{tabular}{|c|c|c|c|c|c|c|c|c|}
\hline Sample Name: & $\begin{array}{c}{ }^{234} \mathbf{U}{ }^{238} \mathbf{U} \\
(\mathbf{p p m}):\end{array}$ & $\mathbf{2 \sigma}(\mathbf{\%}):$ & $\begin{array}{c}{ }^{230} \mathbf{T h} /{ }^{232} \mathbf{T h} \\
(\mathbf{p p m}):\end{array}$ & $\mathbf{2 \sigma}(\%):$ & $\left({ }^{234} \mathbf{U} /{ }^{238} \mathbf{U}\right):$ & $\left({ }^{230} \mathbf{T h} /{ }^{232} \mathbf{T h}\right):$ & $\left({ }^{238} \mathbf{U} /{ }^{232} \mathbf{T h}\right):$ & $\left(^{230} \mathbf{T h}{ }^{238} \mathbf{U}\right):$ \\
\hline OM09-107C-MS-DE \#4 & 65.403 & 0.032 & 25.978 & 0.125 & 1.192 & 4.808 & 14.637 & 0.328 \\
\hline OM09-107C-MS-DE \#5 & 70.110 & 0.022 & 64.542 & 0.059 & 1.277 & 11.946 & 2.215 & 5.393 \\
\hline $\begin{array}{c}\text { OM09-109C-MS-Weak } \\
\text { Acid }\end{array}$ & 63.151 & 0.035 & 9.521 & 0.269 & 1.151 & 1.762 & 2.763 & 0.638 \\
\hline $\begin{array}{c}\text { OM09-109C-MS-Silicate } \\
\text { Residue }\end{array}$ & 57.414 & 0.201 & 57.414 & 0.258 & 1.046 & 10.626 & 0.671 & 15.832 \\
\hline OM09-109C-MS-Total & 61.355 & 0.059 & 7.694 & 0.218 & 1.118 & 1.424 & 1.944 & 0.733 \\
\hline $\begin{array}{c}\text { OM09-10COPS-MS- } \\
\text { Weak Acid }\end{array}$ & 62.035 & 0.052 & 10.747 & 0.182 & 1.130 & 1.989 & 3.652 & 0.545 \\
\hline $\begin{array}{c}\text { OM09-10COPS-MS- } \\
\text { Silicate Residue }\end{array}$ & 59.638 & 0.037 & 6.748 & 0.103 & 1.087 & 1.249 & 0.679 & 1.840 \\
\hline $\begin{array}{c}\text { OM09-10COPS-MS- } \\
\text { Total }\end{array}$ & 61.451 & 0.041 & 7.649 & 0.153 & 1.120 & 1.416 & 1.476 & 0.959 \\
\hline $\begin{array}{c}\text { OM09-84C-MS-Weak } \\
\text { Acid }\end{array}$ & -- & -- & -- & -- & -- & -- & -- & -- \\
\hline OM09-84C-MS-Total & 45.670 & 0.117 & 32.149 & 0.462 & 0.832 & 5.950 & 11.980 & 0.497 \\
\hline
\end{tabular}

Replicate analyses are indicated in italics, with the average of replicate analyses given in bold. Parentheses indicate activities. 


\section{Figures:}

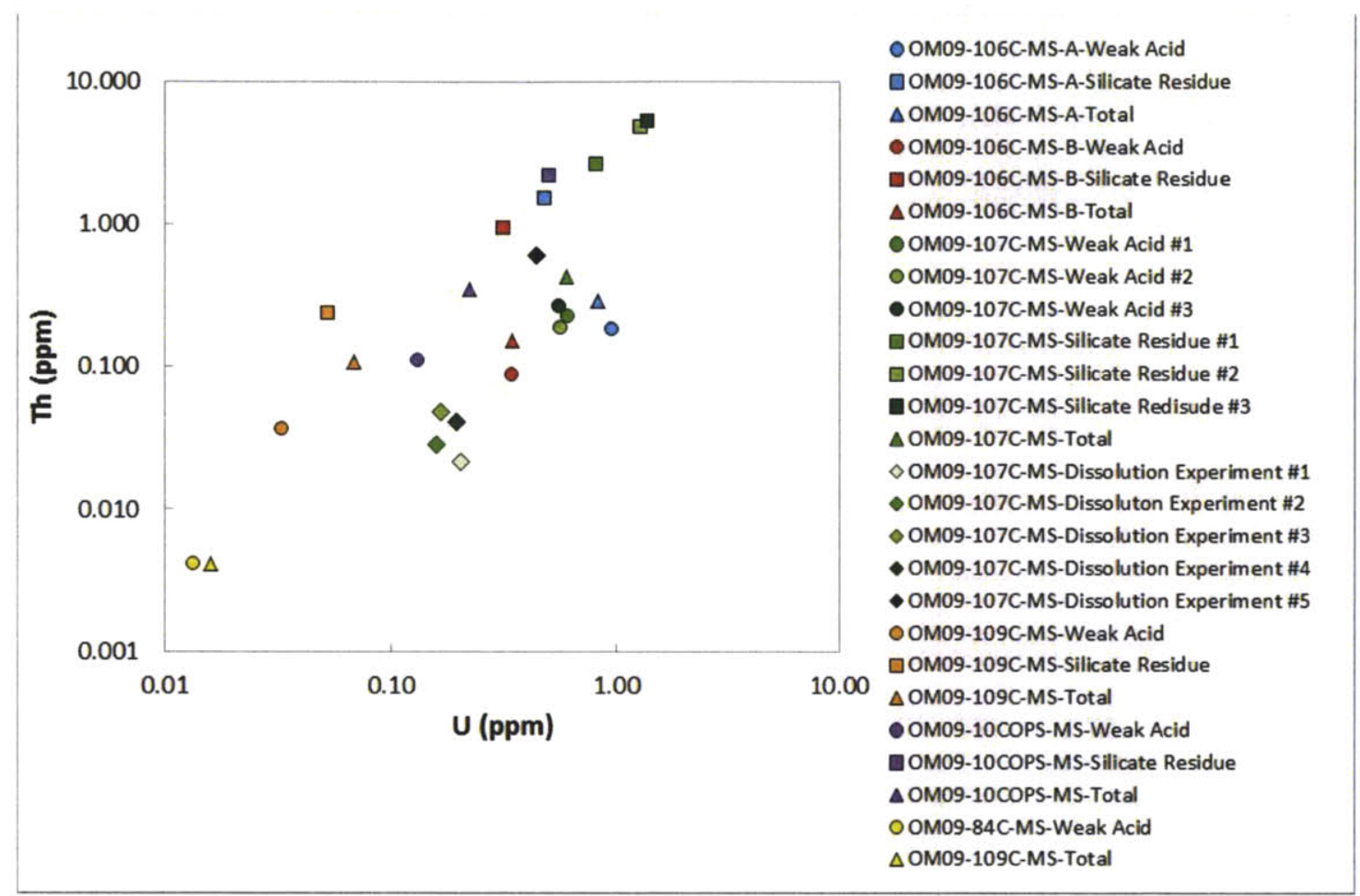

Figure A1: $\mathrm{U}$ and $\mathrm{Th}$ concentrations for various travertine dissolutions: weak acid dissolutions (circles), silicate residues from the weak acid dissolutions (squares), and total dissolutions (triangles). Samples are distinguished by color with the various experiments for OM09-107C-MS distinguished by different shades of green. Internal $2 \sigma$ error bars are smaller than the symbol sizes. Note that both axes have a log scale. 


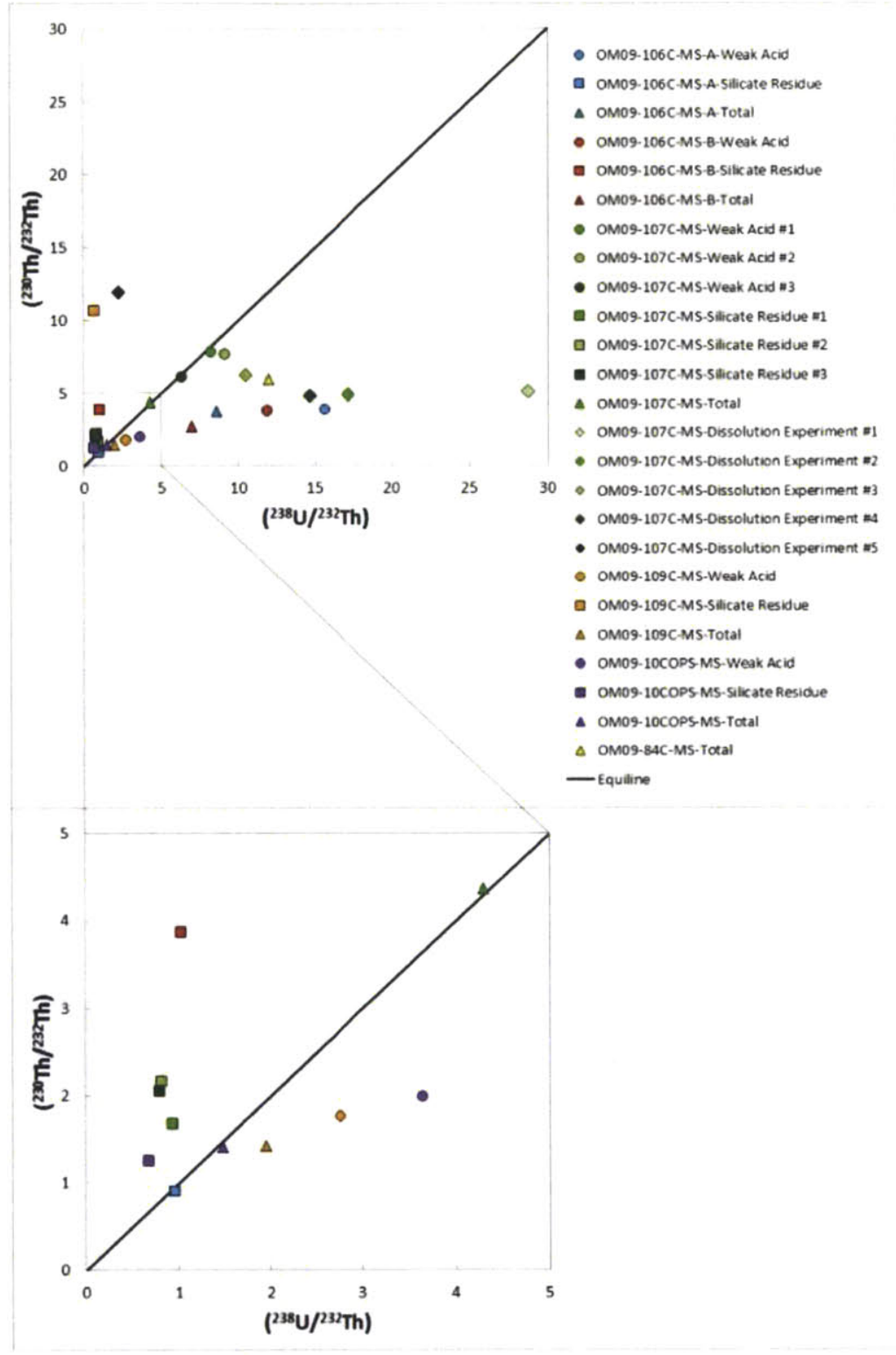

Figure A2: Plot of $\left({ }^{230} \mathrm{Th} /{ }^{232} \mathrm{Th}\right)$ versus $\left({ }^{238} \mathrm{U} /{ }^{232} \mathrm{Th}\right)$ for various travertine dissolutions. The symbols are the same as Figure B1. The parentheses indicate activities. The black line indicates the equiline. Internal $2 \sigma$ error bars are smaller than the symbol sizes. The upper panel displays all of the data while the lower panel enlarges a 5-by-5 section in order to better display the data in the lower-left corner. 


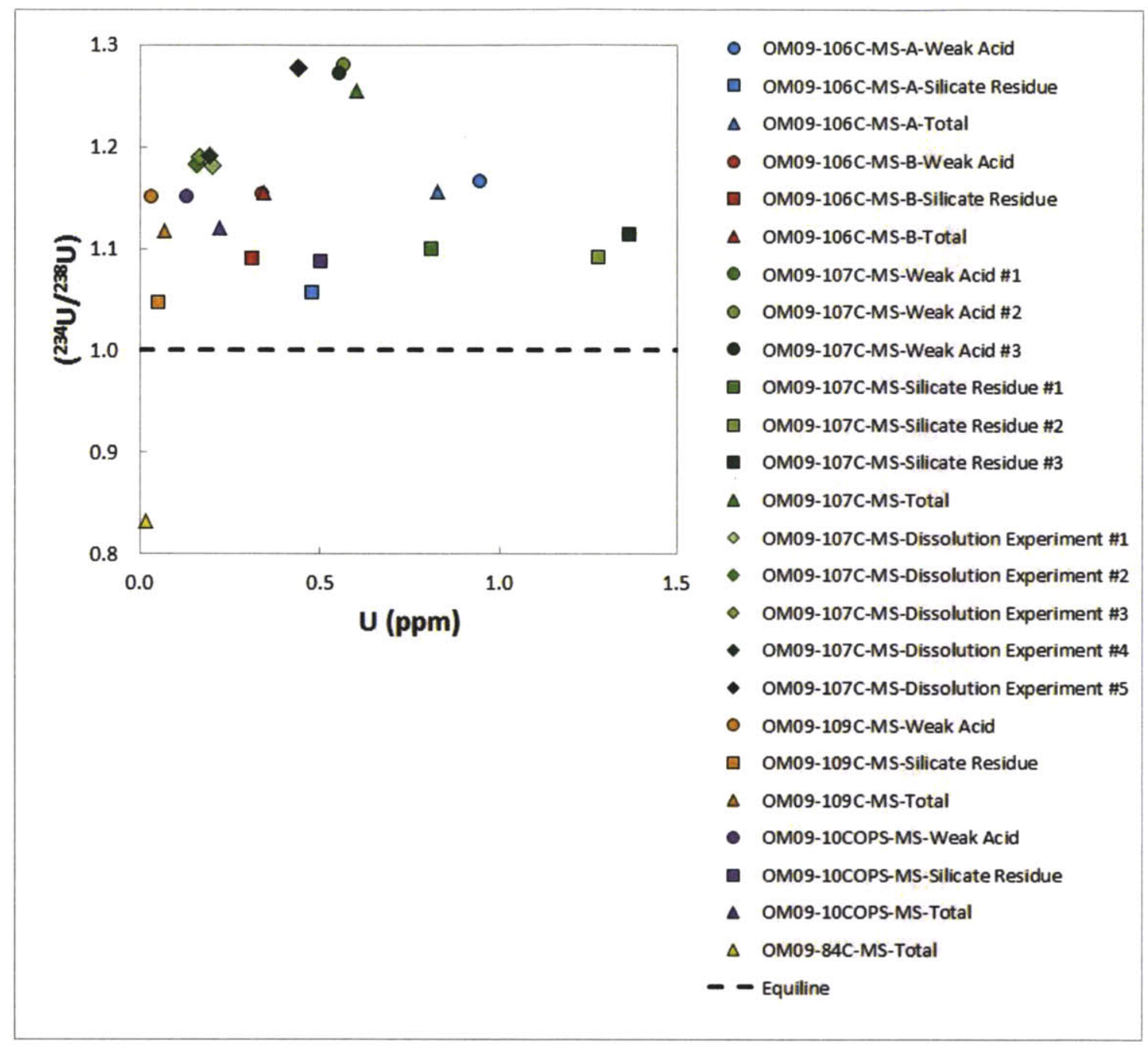

Figure A3: Plot of $\left({ }^{234} \mathrm{U} /{ }^{238} \mathrm{U}\right)$ versus $\mathrm{U}$ concentration for various travertine dissolutions. The symbols are the same as Figure B1. Internal $2 \sigma$ error bars are smaller than the symbol sizes. The dashed line indicates equilibrium for $\left({ }^{234} \mathrm{U} /{ }^{238} \mathrm{U}\right)$. 


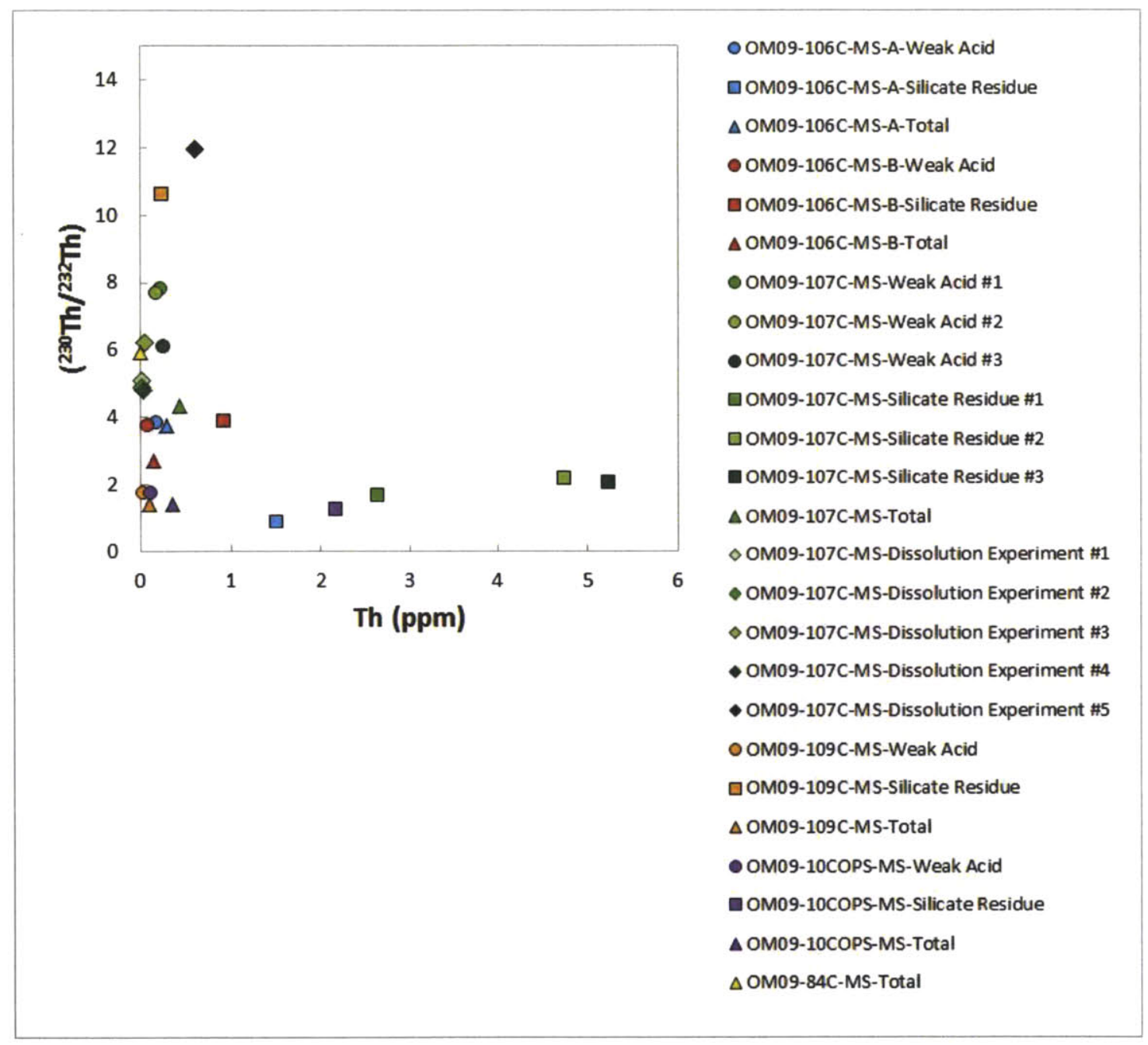

Figure A4: Plot of $\left({ }^{230} \mathrm{Th} /{ }^{232} \mathrm{Th}\right)$ versus $\mathrm{Th}$ concentrations for various travertine dissolutions. The symbols are the same as Figure B1. Internal $2 \sigma$ error bars are smaller than the symbol sizes. 


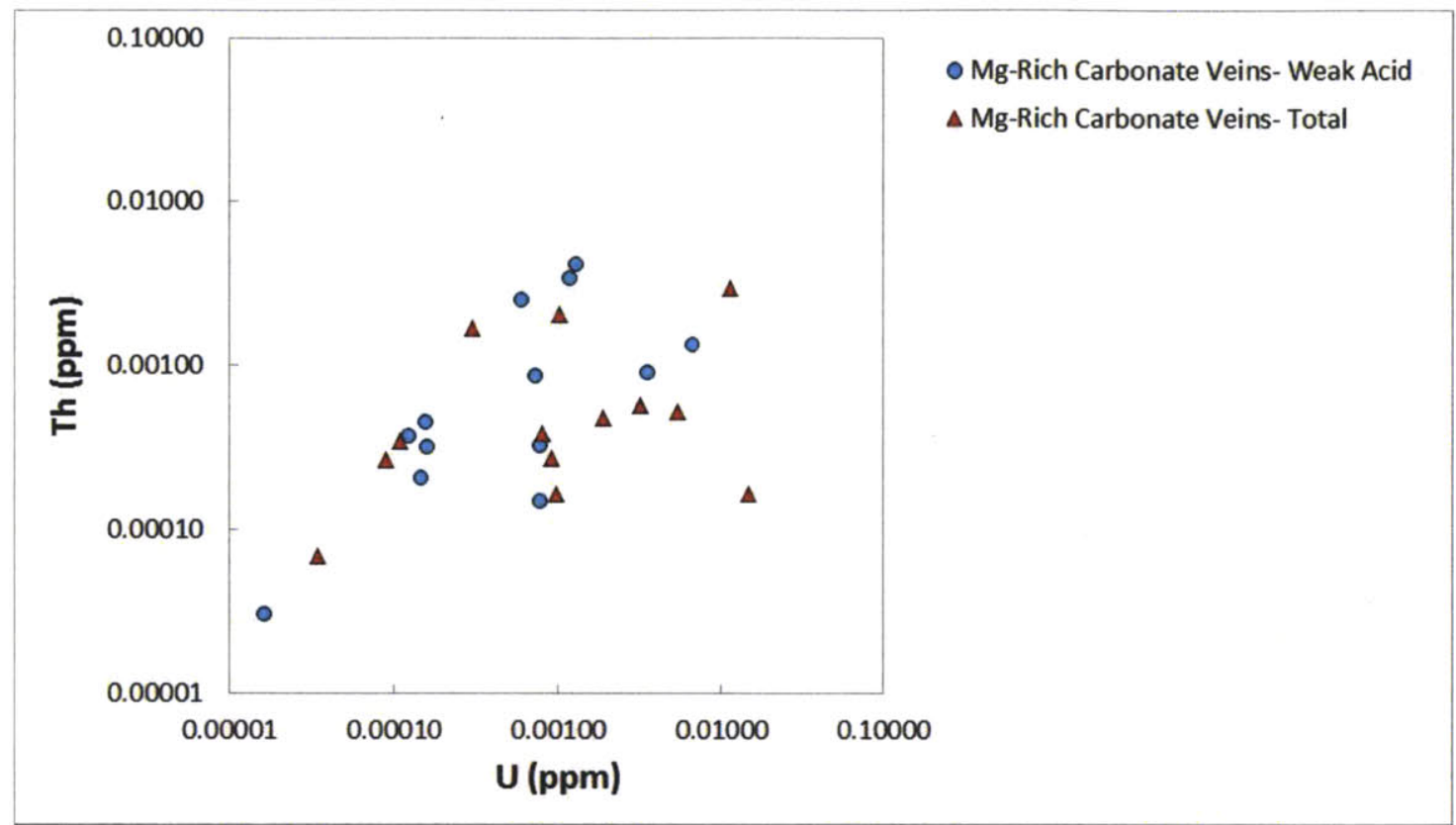

Figure A5: U and Th concentrations for weak acid (blue circles) and total (red triangles) dissolutions of Mg-rich carbonate veins. Internal $2 \sigma$ error bars are smaller than the symbol sizes. Note that both axes have a log scale. 


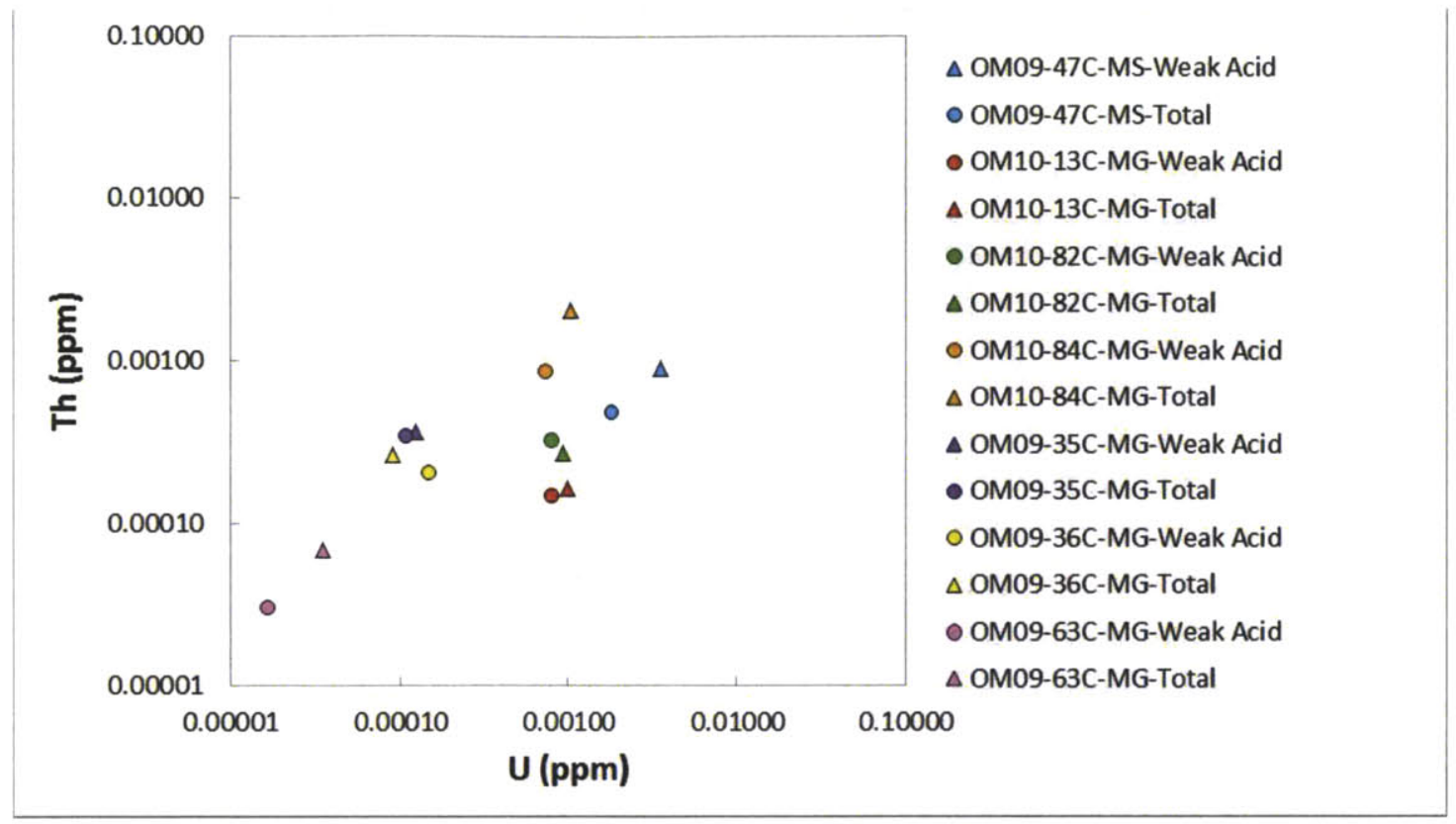

Figure A6: $U$ and $\mathrm{Th}$ concentrations for weak acid (circles) and total (triangles) dissolutions of $\mathrm{Mg}$-rich carbonate veins. Individual samples are distinguished by color. Internal $2 \sigma$ error bars are smaller than the symbol sizes. Note that both axes have a log scale. 


\section{Appendix B: Additional Sensitivity Analysis Figures for Initial ${ }^{230}$ Th Corrections of ${ }^{230}$ Th Ages for Samail Carbonates}

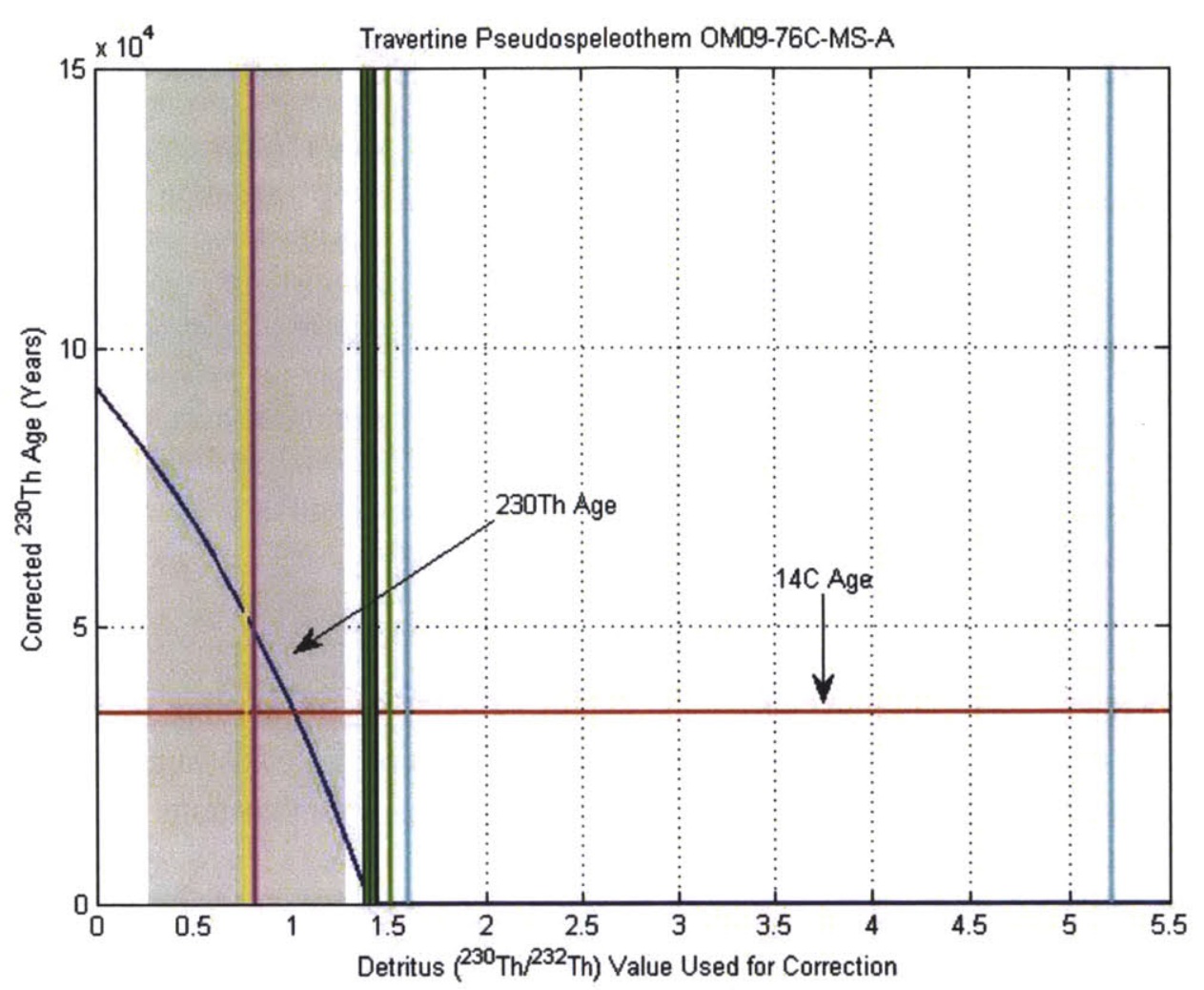

Figure B1: Sensitivity analysis figure showing the effect of the selected $\left({ }^{230} \mathrm{Th} /{ }^{232} \mathrm{Th}\right)$ correction value on the corrected ${ }^{230} \mathrm{Th}$ age calculated using Equation 1 in Chapter 3 . This figure is for Sample OM09-76C-MS-A, a travertine pseudospeleothem from Wadi Sudari Travertine. The dark blue line indicates the corrected ${ }^{230} \mathrm{Th}$ age. Note that the uncorrected age plots at $\mathrm{x}=0$. For comparison, the ${ }^{14} \mathrm{C}$ age is plotted as the red line. The gray box indicates the $2 \sigma$ range of $\left({ }^{230} \mathrm{Th} /{ }^{232} \mathrm{Th}\right)$ for aluminosilicate detritus (literature compilation shown in Chapter 3, Figure 9, $\mathrm{n}=300$ ). Vertical lines indicate possible values to use for the $\left({ }^{230} \mathrm{Th} /{ }^{232} \mathrm{Th}\right)$ correction value: average of aluminosilicate detritus compilation (yellow line), equilibrium Bulk Silicate Earth (pink line), highly-altered peridotites (green lines; note that a fourth peridotite plots off the figure at 12.367), wadi sediments (turquoise lines), and recently-formed travertine precipitates (black lines).The choice of the $\left({ }^{230} \mathrm{Th} /{ }^{232} \mathrm{Th}\right)$ correction value has a very significant impact on the determined ${ }^{230} \mathrm{Th}$ age, which ranges from $\sim 90,000$ years to zero depending on the correction value selected. A $\left({ }^{230} \mathrm{Th} /{ }^{232} \mathrm{Th}\right)$ correction value of approximately 1 brings the corrected ${ }^{230} \mathrm{Th}$ age into concordance with the ${ }^{14} \mathrm{C}$ age. 


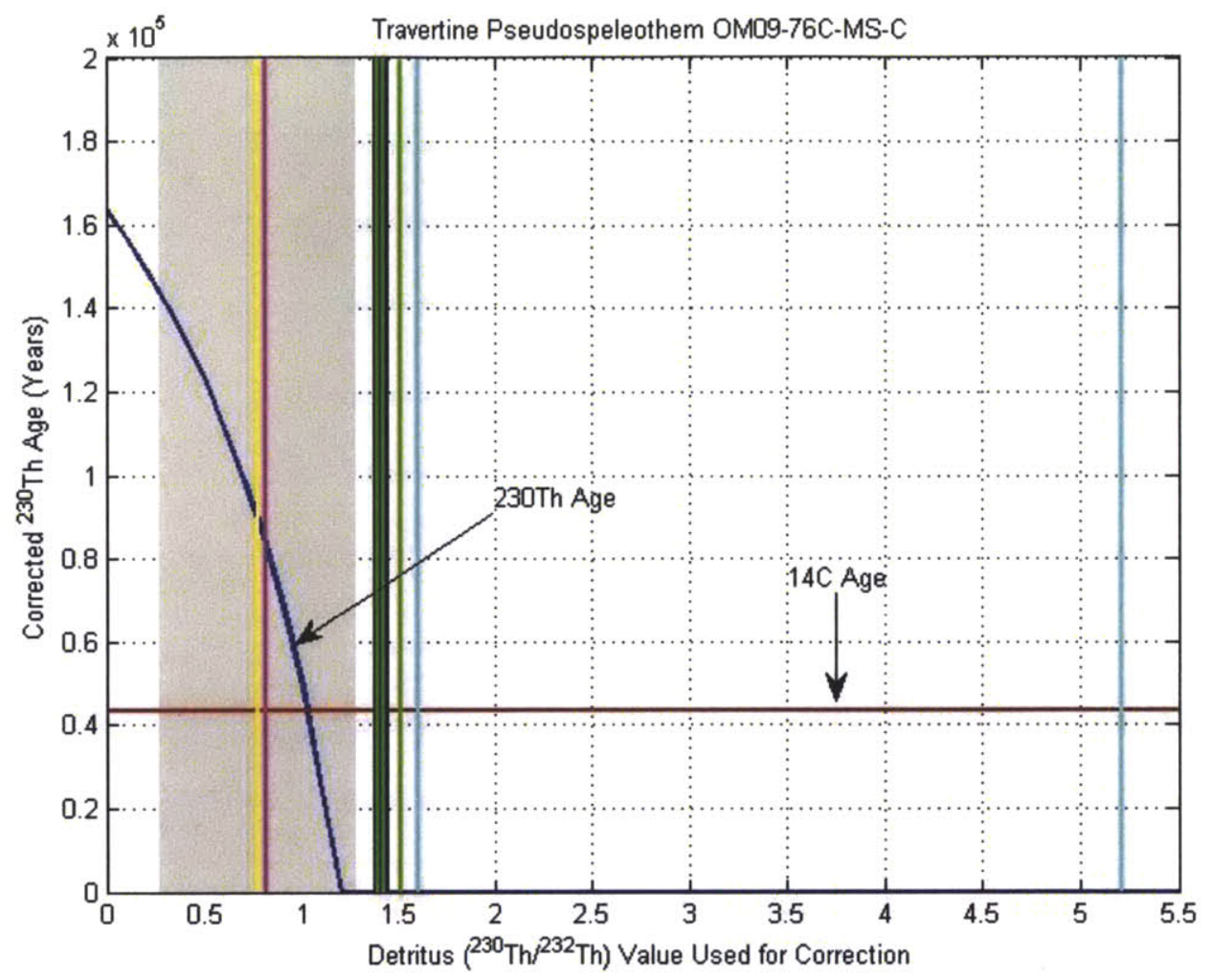

Figure B2: Sensitivity analysis figure showing the effect of the selected $\left({ }^{230} \mathrm{Th} /{ }^{232} \mathrm{Th}\right)$ correction value on the corrected ${ }^{230} \mathrm{Th}$ age calculated using Equation 1 in Chapter 3. This figure is for Sample OM09-76C-MS-C, a travertine pseudospeleothem from Wadi Sudari Travertine. The dark blue line indicates the calculated ${ }^{230} \mathrm{Th}$ age. Note that the uncorrected age plots at $\mathrm{x}=0$. For comparison, the ${ }^{14} \mathrm{C}$ age is plotted as the red line. The gray box indicates the $2 \sigma$ range of $\left({ }^{230} \mathrm{Th} /{ }^{232} \mathrm{Th}\right)$ for aluminosilicate detritus (literature compilation shown in Chapter 3, Figure 9, $\mathrm{n}=300$ ). Vertical lines indicate possible values to use for the $\left({ }^{230} \mathrm{Th} /{ }^{232} \mathrm{Th}\right)$ correction value: average of aluminosilicate detritus compilation (yellow line), equilibrium Bulk Silicate Earth (pink line), highly-altered peridotites (green lines; note that a fourth peridotite plots off the figure at 12.367), wadi sediments (turquoise lines), and recently-formed travertine precipitates (black lines). The choice of the $\left({ }^{230} \mathrm{Th} /{ }^{232} \mathrm{Th}\right)$ correction value has a very significant impact on the determined ${ }^{230} \mathrm{Th}$ age, which ranges from $>100,000$ years to zero depending on the correction value selected. A $\left({ }^{230} \mathrm{Th} /{ }^{232} \mathrm{Th}\right)$ correction value of approximately 1 brings the corrected ${ }^{230} \mathrm{Th}$ age into concordance with the ${ }^{14} \mathrm{C}$ age. 


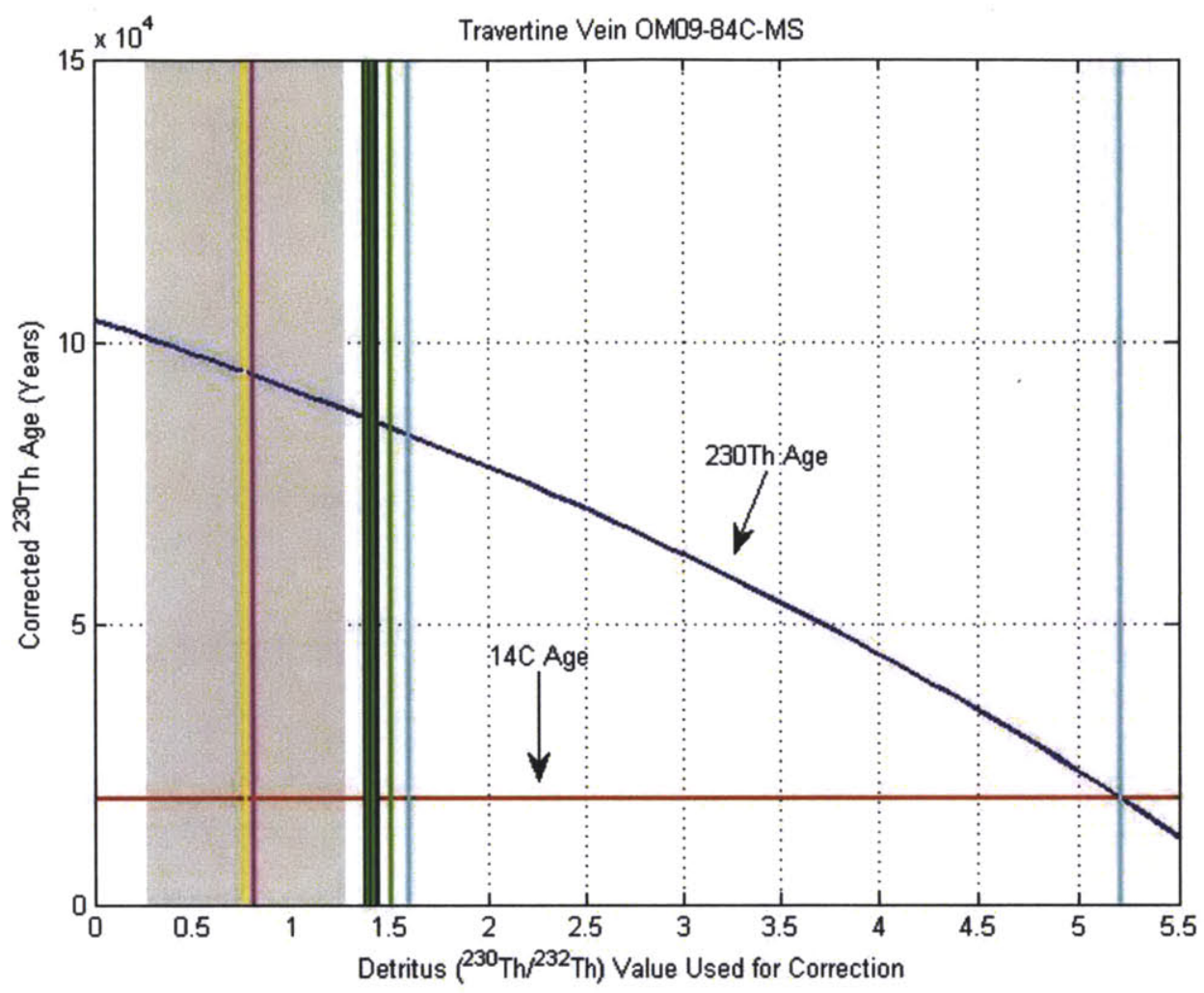

Figure B3: Sensitivity analysis figure showing the effect of the selected $\left({ }^{230} \mathrm{Th} /{ }^{232} \mathrm{Th}\right)$ correction value on the correction ${ }^{230} \mathrm{Th}$ age calculated using Equation 1 in Chapter 3. This figure is for Sample OM09-84C-MS, a travertine vein from Wadi Sudari Travertine. The dark blue line indicates the calculated ${ }^{230} \mathrm{Th}$ age. Note that the uncorrected age plots at $\mathrm{x}=0$. For comparison, the ${ }^{14} \mathrm{C}$ age is plotted as the red line. The gray box indicates the $2 \sigma$ range of $\left({ }^{230} \mathrm{Th} /{ }^{232} \mathrm{Th}\right.$ ) for aluminosilicate detritus (literature compilation shown in Chapter 3, Figure 9, $\mathrm{n}=300$ ). Vertical lines indicate possible values to use for the initial $\left({ }^{230} \mathrm{Th} /{ }^{232} \mathrm{Th}\right)$ : average of aluminosilicate detritus compilation (yellow line), equilibrium Bulk Silicate Earth (pink line), highly-altered peridotites (green lines; note that a fourth peridotite plots off the figure at 12.367), wadi sediments (turquoise lines), and recentlyformed travertine precipitates (black lines).The choice of the $\left({ }^{230} \mathrm{Th} /{ }^{232} \mathrm{Th}\right)$ correction value has a very significant impact on the determined ${ }^{230} \mathrm{Th}$ age, which ranges from $\sim 100,000$ years to zero depending on the correction value selected. $\mathrm{A}\left({ }^{230} \mathrm{Th} /{ }^{232} \mathrm{Th}\right)$ correction value of approximately 5.2 brings the corrected ${ }^{230} \mathrm{Th}$ age into concordance with the ${ }^{14} \mathrm{C}$ age. 


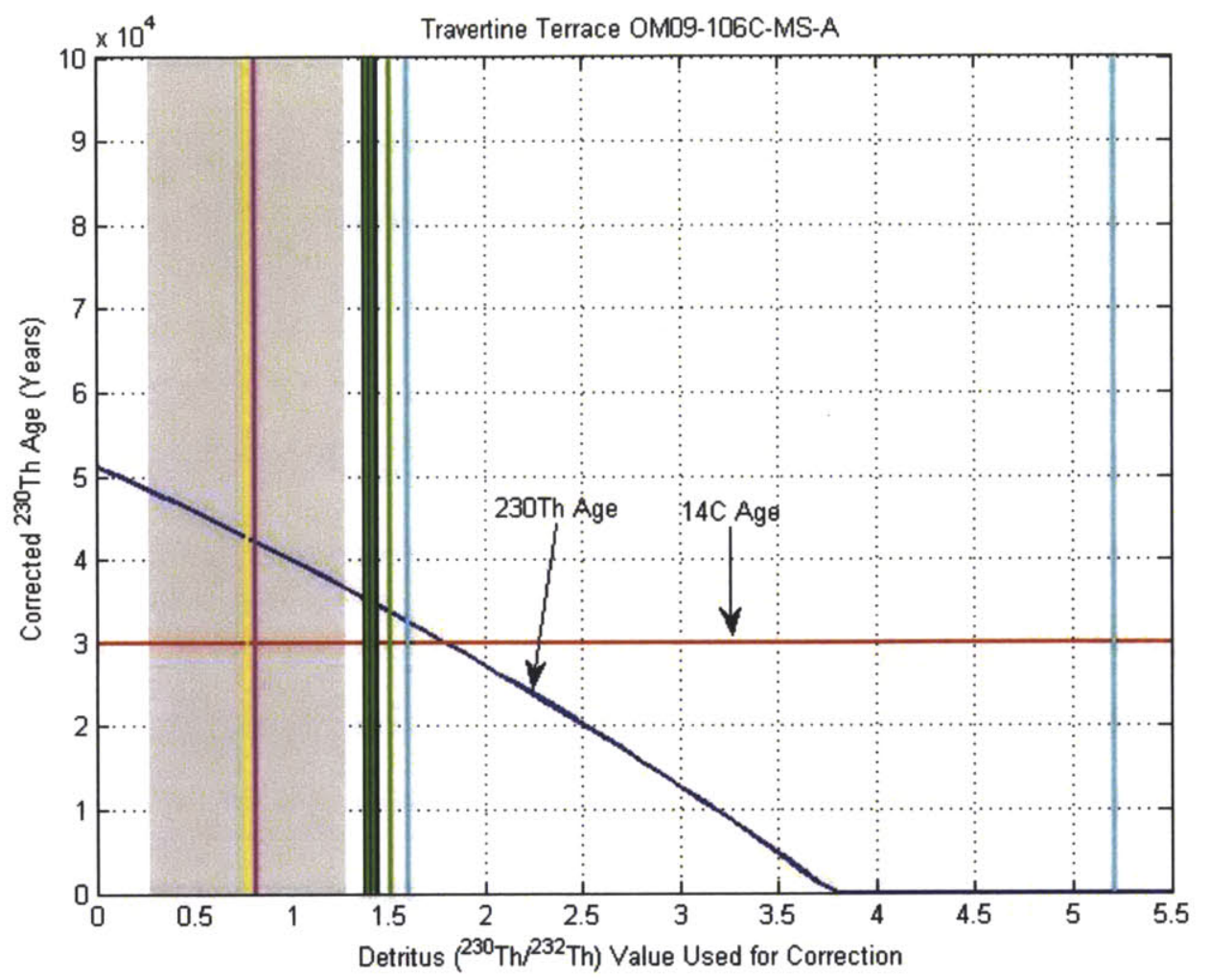

Figure B4: Sensitivity analysis figure showing the effect of the selected $\left({ }^{230} \mathrm{Th} /{ }^{232} \mathrm{Th}\right)$ correction value on the corrected ${ }^{230} \mathrm{Th}$ age calculated using Equation 1. This figure is for travertine Sample OM09-106C-MS-A, a travertine terrace from Wadi Uqaybah. The dark blue line indicates the corrected ${ }^{230} \mathrm{Th}$ age. Note that the uncorrected ${ }^{230} \mathrm{Th}$ age plots at $\mathrm{x}$ $=0$. For comparison, the ${ }^{14} \mathrm{C}$ is plotted as the red line. The gray box indicates the $2 \sigma$ range of $\left({ }^{230} \mathrm{Th} /{ }^{232} \mathrm{Th}\right)$ for aluminosilicate detritus (literature compilation shown in Figure $9, \mathrm{n}=300)$. Vertical lines indicate possible values to use for the $\left({ }^{230} \mathrm{Th} /{ }^{232} \mathrm{Th}\right)$ correction value: average of aluminosilicate detritus compilation (yellow line), equilibrium Bulk Silicate Earth (pink line), highly-altered peridotites (green lines; note that a fourth peridotite plots off the figure at 12.367), wadi sediments (turquoise lines), and recentlyformed travertine precipitates (black lines). The choice of the $\left({ }^{230} \mathrm{Th} /{ }^{232} \mathrm{Th}\right)$ correction value has a very significant impact on the determined ${ }^{230} \mathrm{Th}$ age, which ranges from $\sim 50,000$ years to zero depending on the correction value selected. However, a $\left({ }^{230} \mathrm{Th} /{ }^{232} \mathrm{Th}\right)$ correction value of approximately 1.7 brings the corrected ${ }^{230} \mathrm{Th}$ age into concordance with the ${ }^{14} \mathrm{C}$ age. 


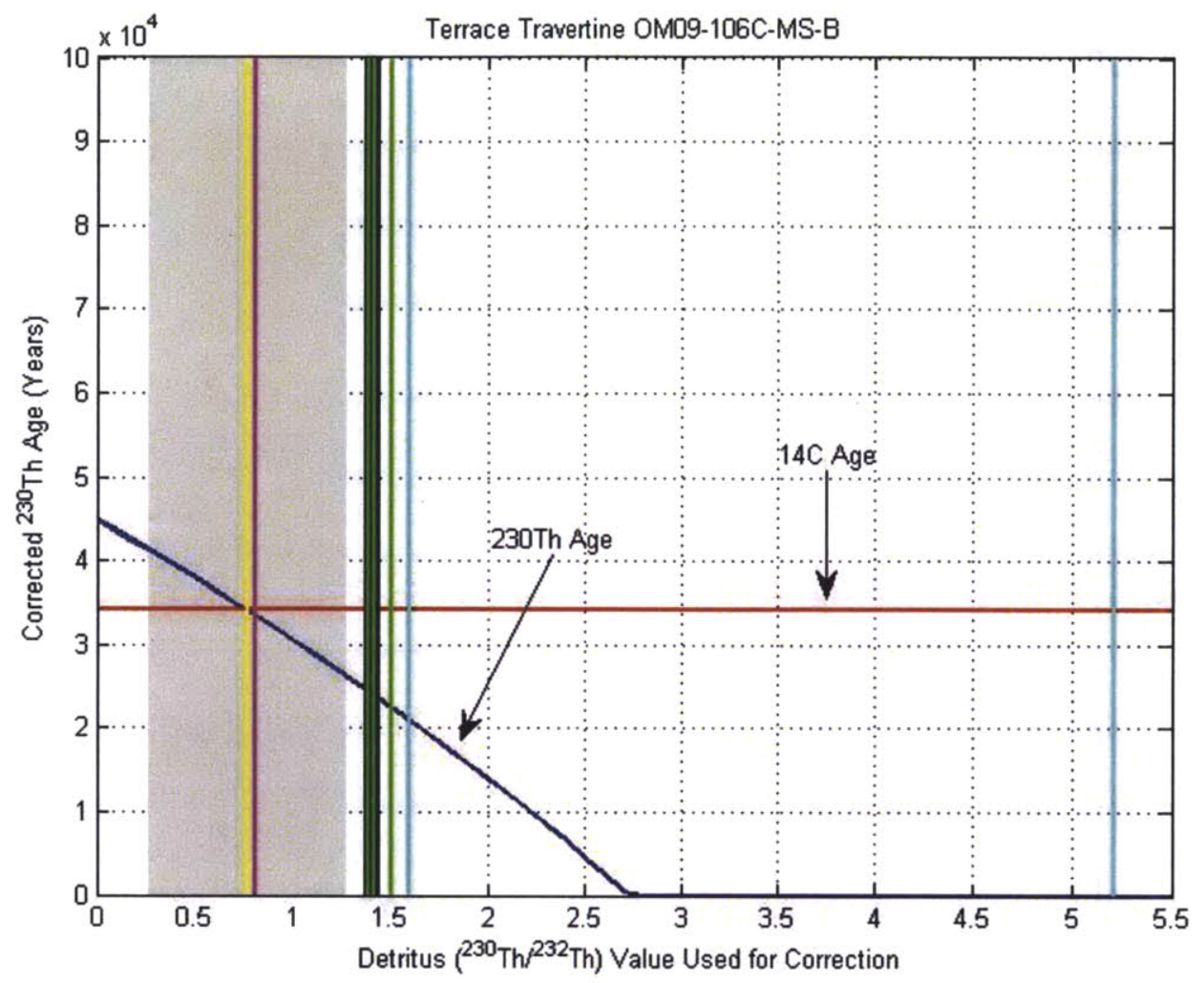

Figure B5: Sensitivity analysis figure showing the effect of the selected $\left({ }^{230} \mathrm{Th} /{ }^{232} \mathrm{Th}\right)$ correction value on the corrected ${ }^{230} \mathrm{Th}$ age calculated using Equation 1 in Chapter 3 . This figure is for Sample OM09-106C-MS-B, a travertine terrace from Wadi Uqaybah Travertine. The dark blue line indicates the calculated ${ }^{230} \mathrm{Th}$ age. Note that the uncorrected age plots at $\mathrm{x}=0$. For comparison, the ${ }^{14} \mathrm{C}$ age is plotted as the red line. The gray box indicates the $2 \sigma$ range of $\left({ }^{230} \mathrm{Th} /{ }^{232} \mathrm{Th}\right)$ for aluminosilicate detritus (literature compilation shown in Chapter 3, Figure 9, $\mathrm{n}=300$ ). Vertical lines indicate possible values to use for the initial $\left({ }^{230} \mathrm{Th} /{ }^{232} \mathrm{Th}\right)$ : average of aluminosilicate detritus compilation (yellow line), equilibrium Bulk Silicate Earth (pink line), highly-altered peridotites (green lines; note that a fourth peridotite plots off the figure at 12.367), wadi sediments (turquoise lines), and recently-formed travertine precipitates (black lines).The choice of the $\left({ }^{230} \mathrm{Th} /{ }^{232} \mathrm{Th}\right)$ correction value has a very significant impact on the determined ${ }^{230} \mathrm{Th}$ age, which ranges from $\sim 45,000$ years to zero depending on the correction value selected. $\mathrm{A}\left({ }^{230} \mathrm{Th} /{ }^{232} \mathrm{Th}\right)$ correction value of approximately 1.7 brings the corrected ${ }^{230} \mathrm{Th}$ age into concordance with the ${ }^{14} \mathrm{C}$ age. 


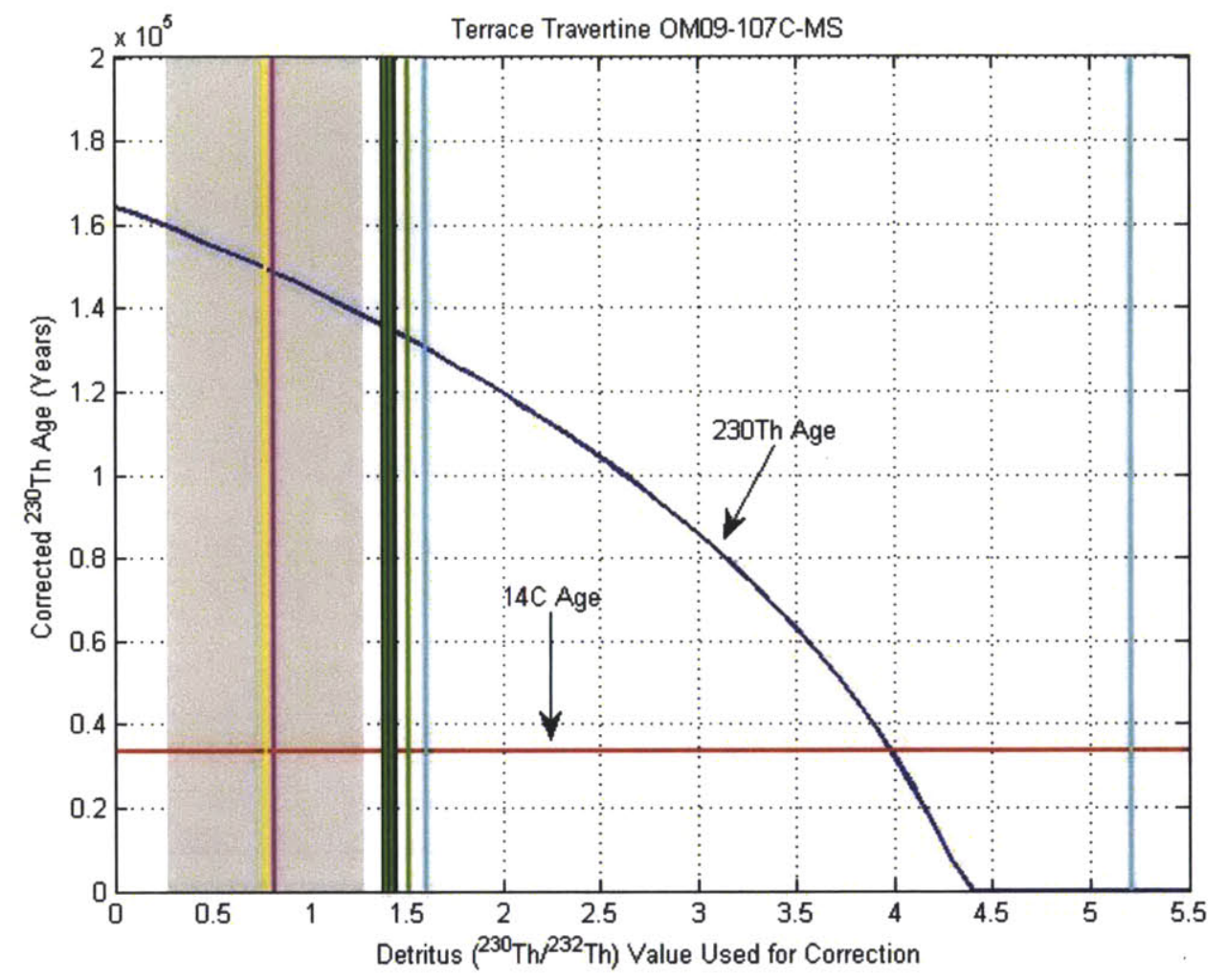

Figure B6: Sensitivity analysis figure showing the effect of the selected $\left({ }^{230} \mathrm{Th} /{ }^{232} \mathrm{Th}\right)$ correction value on the corrected ${ }^{230} \mathrm{Th}$ age calculated using Equation 1 in Chapter 3. This figure is for Sample OM09-107C-MS-C, a travertine terrace from Wadi Uqaybah Travertine. The dark blue line indicates the calculated ${ }^{230} \mathrm{Th}$ age. Note that the uncorrected age plots at $\mathrm{x}=0$. For comparison, the ${ }^{14} \mathrm{C}$ age is plotted as the red line. The gray box indicates the $2 \sigma$ range of $\left({ }^{230} \mathrm{Th} /{ }^{232} \mathrm{Th}\right.$ ) for aluminosilicate detritus (literature compilation shown in Chapter 3, Figure 9, $\mathrm{n}=300$ ). Vertical lines indicate possible values to use for the initial $\left({ }^{230} \mathrm{Th} /{ }^{232} \mathrm{Th}\right)$ : average of aluminosilicate detritus compilation (yellow line), equilibrium Bulk Silicate Earth (pink line), highly-altered peridotites (green lines; note that a fourth peridotite plots off the figure at 12.367), wadi sediments (turquoise lines), and recently-formed travertine precipitates (black lines).The choice of the $\left({ }^{230} \mathrm{Th} /{ }^{232} \mathrm{Th}\right)$ correction value has a very significant impact on the determined ${ }^{230} \mathrm{Th}$ age, which ranges from $\sim 160,000$ years to zero depending on the correction value selected. A $\left({ }^{230} \mathrm{Th} /{ }^{232} \mathrm{Th}\right)$ correction value of approximately 4 brings the corrected ${ }^{230} \mathrm{Th}$ into concordance with the ${ }^{14} \mathrm{C}$ age. 


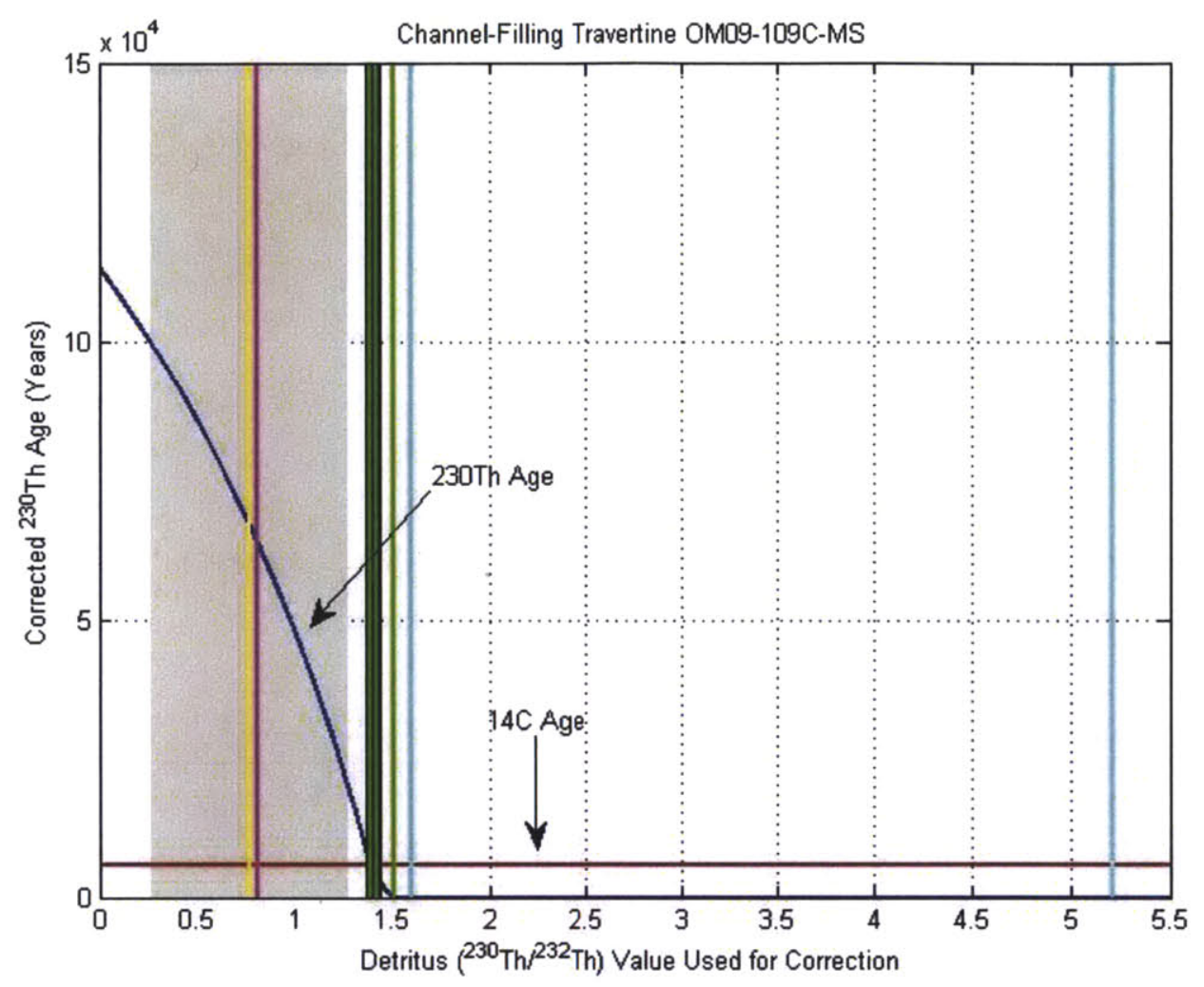

Figure B7: Sensitivity analysis figure showing the effect of the $\left({ }^{230} \mathrm{Th} /{ }^{232} \mathrm{Th}\right)$ correction value on the corrected ${ }^{230} \mathrm{Th}$ age calculated using Equation 1 in Chapter 3. This figure is for Sample OM09-109C-MS-C, a travertine terrace from Wadi Uqaybah Travertine. The dark blue line indicates the calculated ${ }^{230} \mathrm{Th}$ age. Note that the uncorrected age plots at $\mathrm{x}$ $=0$. For comparison, the ${ }^{14} \mathrm{C}$ age is plotted as the red line. The gray box indicates the $2 \sigma$ range of $\left({ }^{230} \mathrm{Th} /{ }^{232} \mathrm{Th}\right)$ for aluminosilicate detritus (literature compilation shown in Chapter 3, Figure 9, $\mathrm{n}=300$ ). Vertical lines indicate possible values to use for the initial $\left({ }^{230} \mathrm{Th} /{ }^{232} \mathrm{Th}\right)$ : average of aluminosilicate detritus compilation (yellow line), equilibrium Bulk Silicate Earth (pink line), highly-altered peridotites (green lines; note that a fourth peridotite plots off the figure at 12.367), wadi sediments (turquoise lines), and recentlyformed travertine precipitates (black lines). The choice of the $\left({ }^{230} \mathrm{Th} /{ }^{232} \mathrm{Th}\right)$ correction value has a very significant impact on the determined ${ }^{230} \mathrm{Th}$ age, which ranges from $>100,000$ years to zero depending on the correction value selected. A $\left({ }^{230} \mathrm{Th} /{ }^{232} \mathrm{Th}\right)$ correction value of approximately 1.5 brings the corrected ${ }^{230} \mathrm{Th}$ age into concordance with the ${ }^{14} \mathrm{C}$ age. 


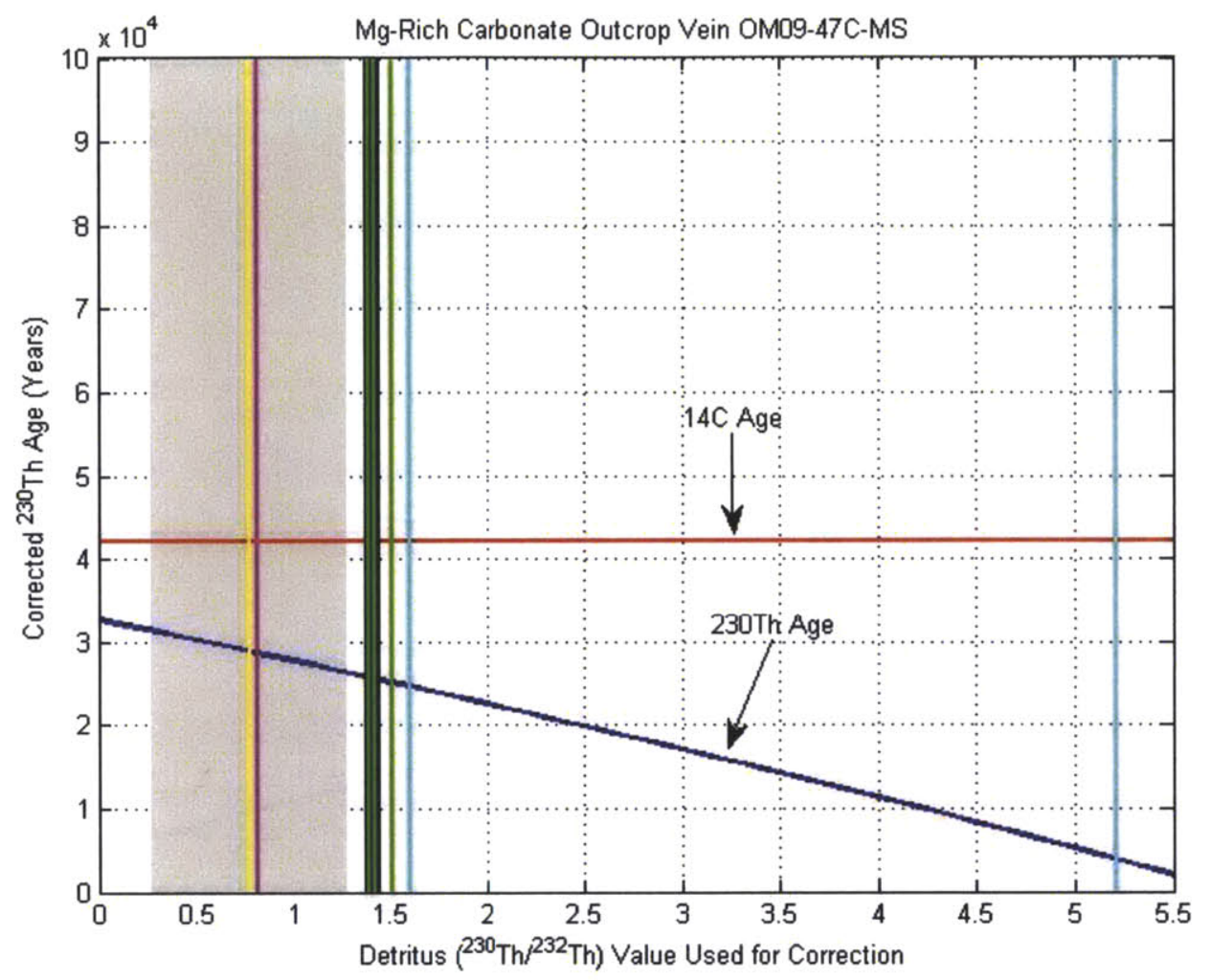

Figure B8: Sensitivity analysis figure showing the effect of the $\left({ }^{230} \mathrm{Th} /{ }^{232} \mathrm{Th}\right)$ correction value on the corrected ${ }^{230} \mathrm{Th}$ age calculated using Equation 1 in Chapter 3. This figure is for Sample OM09-47C-MS, an Mg-rich carbonate outcroup vein from Fanja Roadcut. The dark blue line indicates the calculated ${ }^{230} \mathrm{Th}$ age. Note that the uncorrected age plots at $\mathrm{x}=0$. For comparison, the ${ }^{14} \mathrm{C}$ age is plotted as the red line. The gray box indicates the $2 \sigma$ range of $\left({ }^{230} \mathrm{Th} /{ }^{232} \mathrm{Th}\right)$ for aluminosilicate detritus (literature compilation shown in Chapter 3, Figure 9, $n=300$ ). Vertical lines indicate possible values to use for the initial $\left({ }^{230} \mathrm{Th}{ }^{232} \mathrm{Th}\right)$ : average of aluminosilicate detritus compilation (yellow line), equilibrium Bulk Silicate Earth (pink line), highly-altered peridotites (green lines; note that a fourth peridotite plots off the figure at 12.367), wadi sediments (turquoise lines), and recentlyformed travertine precipitates (black lines).The choice of the $\left({ }^{230} \mathrm{Th} /{ }^{232} \mathrm{Th}\right)$ correction value has a very significant impact on the determined ${ }^{230} \mathrm{Th}$ age, which ranges from $\sim 20,000$ years to zero depending on the correction value selected. Since the uncorrected ${ }^{230} \mathrm{Th}$ age is younger than the ${ }^{14} \mathrm{C}$ age, no ${ }^{230} \mathrm{Th}$ correction can bring the two ages into concordance. 


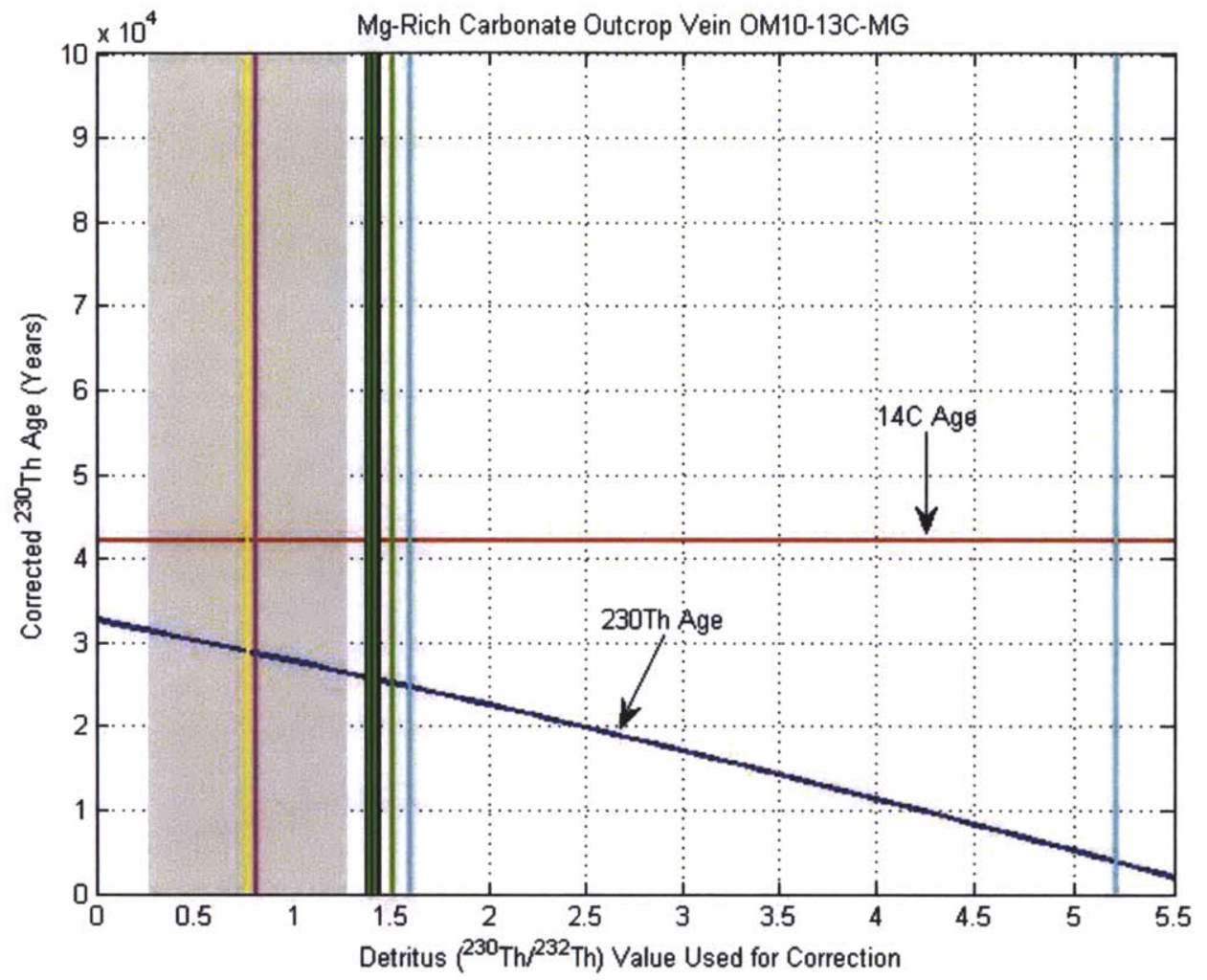

Figure B9: Sensitivity analysis figure showing the effect of the $\left({ }^{230} \mathrm{Th} /{ }^{232} \mathrm{Th}\right)$ correction value on the corrected ${ }^{230} \mathrm{Th}$ age calculated using Equation 1. This figure is for Sample OM10-13C-MG, an Mg-rich carbonate outcroup vein from Fanja Roadcut. The dark blue line indicates the corrected ${ }^{230} \mathrm{Th}$ age. Note that the uncorrected ${ }^{230} \mathrm{Th}$ age plots at $\mathrm{x}=0$. For comparison, the ${ }^{14} \mathrm{C}$ age is plotted as the red line. The gray box indicates the $2 \sigma$ range of $\left({ }^{230} \mathrm{Th} /{ }^{232} \mathrm{Th}\right)$ for aluminosilicate detritus (literature compilation shown in Figure $9, \mathrm{n}=$ 300). Vertical lines indicate possible values to use for the $\left({ }^{230} \mathrm{Th} /{ }^{232} \mathrm{Th}\right)$ correction value: average of aluminosilicate detritus compilation (yellow line), equilibrium Bulk Silicate Earth (pink line), highly-altered peridotites (green lines; note that a fourth peridotite plots off the figure at 12.367), wadi sediments (turquoise lines), and recently-formed travertine precipitates (black lines). The choice of the $\left({ }^{230} \mathrm{Th} /{ }^{232} \mathrm{Th}\right)$ correction value has a very significant impact on the determined ${ }^{230} \mathrm{Th}$ age, which ranges from $\sim 30,000$ years to zero depending on the correction value selected. Since the uncorrected ${ }^{230} \mathrm{Th}$ age is younger than the ${ }^{14} \mathrm{C}$ age, no ${ }^{230} \mathrm{Th}$ correction can bring the two ages into concordance. 


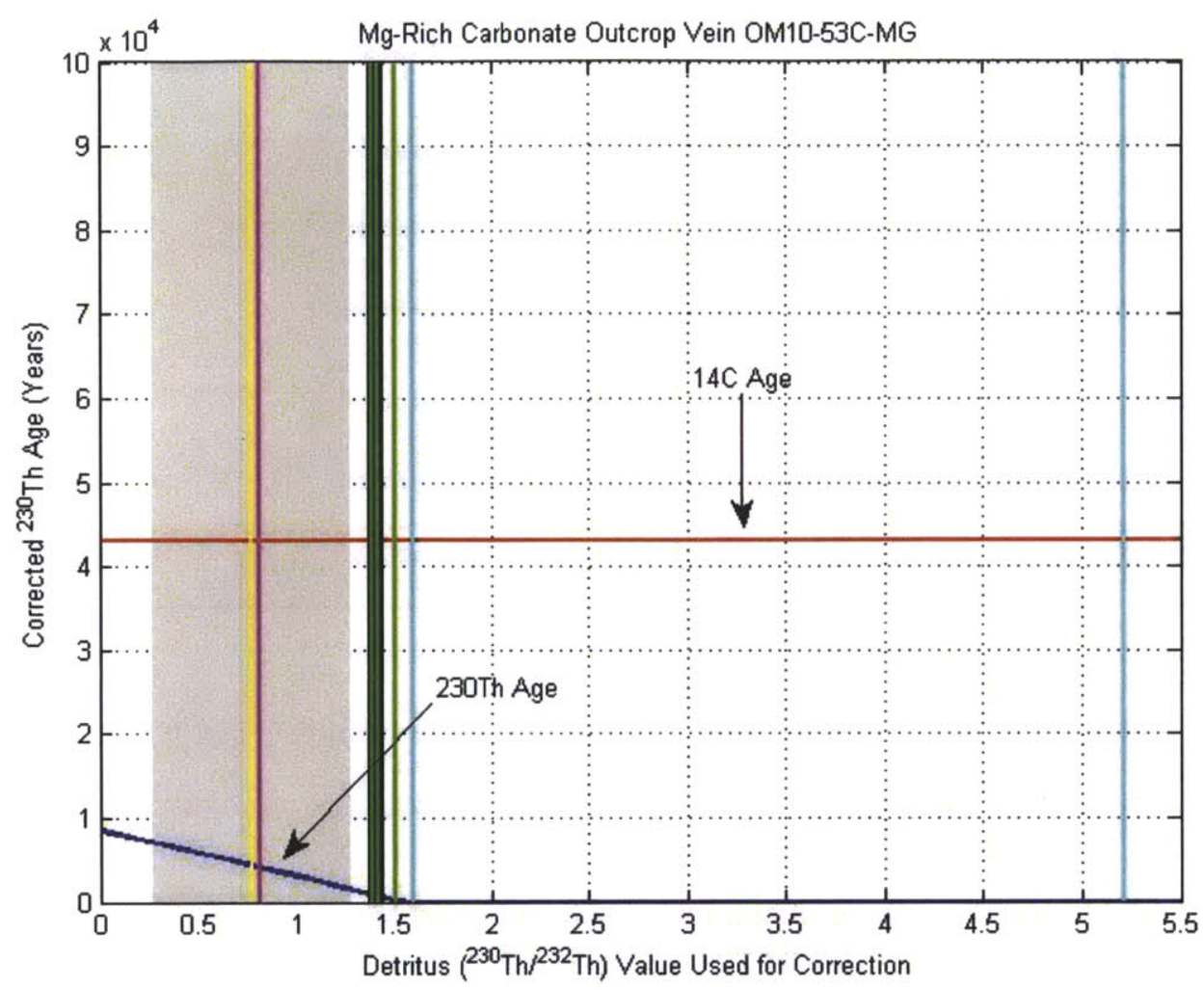

Figure B10: Sensitivity analysis figure showing the effect of the $\left({ }^{230} \mathrm{Th} /{ }^{232} \mathrm{Th}\right)$ correction value on the corrected ${ }^{230} \mathrm{Th}$ age calculated using Equation 1 in Chapter 3. This figure is for Sample OM10-53C-MG, an Mg-rich carbonate outcroup vein from Al-Wuqbah Roadcut. The dark blue line indicates the calculated ${ }^{230} \mathrm{Th}$ age. Note that the uncorrected age plots at $\mathrm{x}=0$. For comparison, the ${ }^{14} \mathrm{C}$ age is plotted as the red line. The gray box indicates the $2 \sigma$ range of $\left({ }^{230} \mathrm{Th} /{ }^{232} \mathrm{Th}\right)$ for aluminosilicate detritus (literature compilation shown in Chapter 3, Figure 9, $n=300$ ). Vertical lines indicate possible values to use for the initial $\left({ }^{230} \mathrm{Th} /{ }^{232} \mathrm{Th}\right)$ : average of aluminosilicate detritus compilation (yellow line), equilibrium Bulk Silicate Earth (pink line), highly-altered peridotites (green lines; note that a fourth peridotite plots off the figure at 12.367), wadi sediments (turquoise lines), and recently-formed travertine precipitates (black lines). The choice of the $\left({ }^{230} \mathrm{Th} /{ }^{232} \mathrm{Th}\right)$ correction value has a very significant impact on the determined ${ }^{230} \mathrm{Th}$ age, which ranges from $\sim 10,000$ years to zero depending on the correction value selected. Since the uncorrected ${ }^{230} \mathrm{Th}$ age is younger than the ${ }^{14} \mathrm{C}$ age, no ${ }^{230} \mathrm{Th}$ correction can bring the two ages into concordance. 


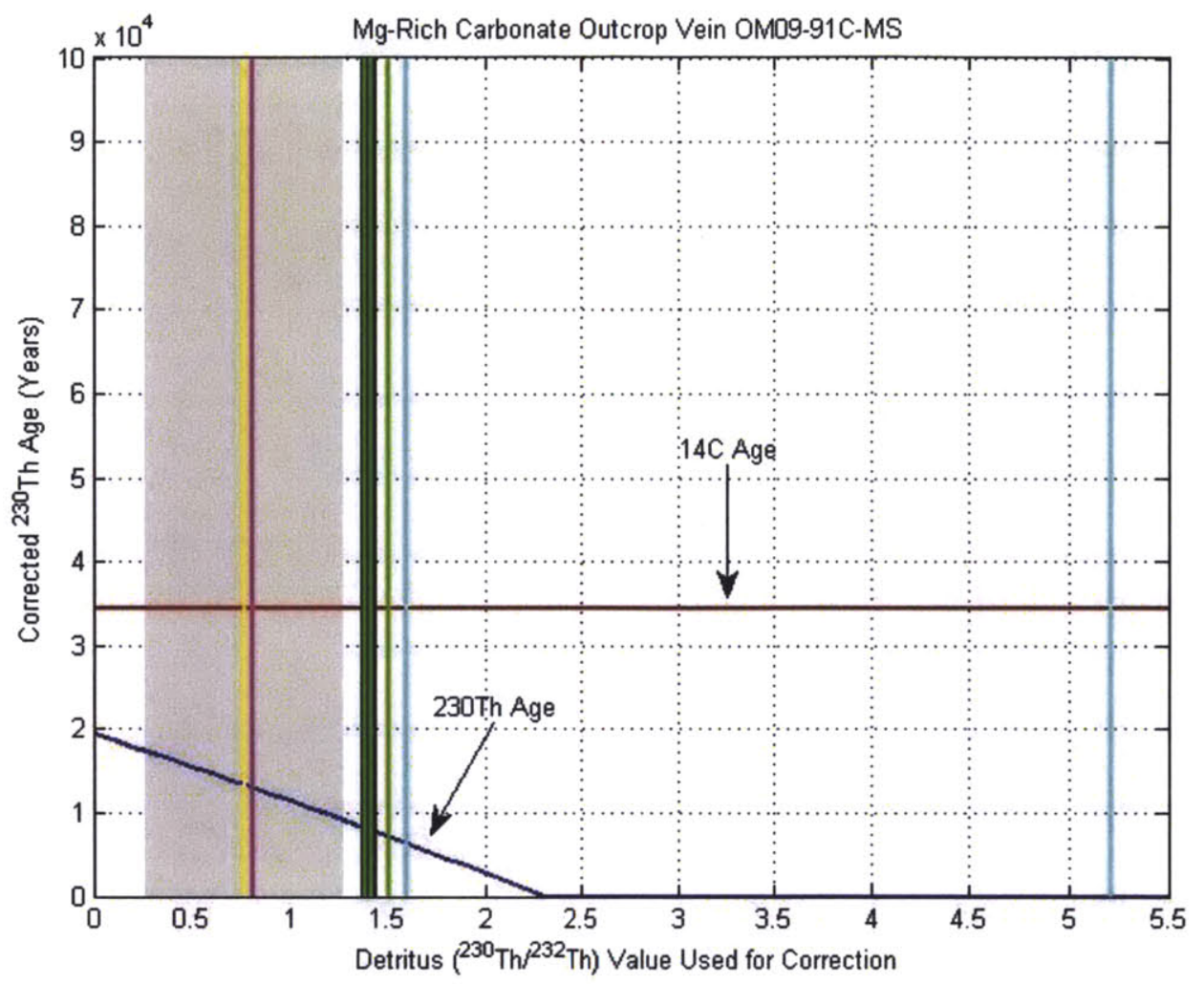

Figure B11: Sensitivity analysis figure showing the effect of the $\left({ }^{230} \mathrm{Th} /{ }^{232} \mathrm{Th}\right)$ correction value on the corrected ${ }^{230} \mathrm{Th}$ age calculated using Equation 1 in Chapter 3 . This figure is for Sample OM09-91C-MS, an Mg-rich carbonate outcroup vein from Wadi Sudari Campsite. The dark blue line indicates the calculated ${ }^{230} \mathrm{Th}$ age. Note that the uncorrected age plots at $\mathrm{x}=0$. For comparison, the ${ }^{14} \mathrm{C}$ age is plotted as the red line. The gray box indicates the $2 \sigma$ range of $\left({ }^{230} \mathrm{Th} /{ }^{232} \mathrm{Th}\right.$ ) for aluminosilicate detritus (literature compilation shown in Chapter 3, Figure 9, $\mathrm{n}=300$ ). Vertical lines indicate possible values to use for the initial $\left({ }^{230} \mathrm{Th} /{ }^{232} \mathrm{Th}\right)$ : average of aluminosilicate detritus compilation (yellow line), equilibrium Bulk Silicate Earth (pink line), highly-altered peridotites (green lines; note that a fourth peridotite plots off the figure at 12.367), wadi sediments (turquoise lines), and recently-formed travertine precipitates (black lines). The choice of the $\left({ }^{230} \mathrm{Th} /{ }^{232} \mathrm{Th}\right.$ ) correction has a very significant impact on the determined ${ }^{230} \mathrm{Th}^{238} \mathrm{U}$ age, which ranges from $\sim 20,000$ years to zero depending on the correction value selected. Since the uncorrected ${ }^{230} \mathrm{Th}$ age is younger than the ${ }^{14} \mathrm{C}$ age, no ${ }^{230} \mathrm{Th}$ correction can bring the two ages into concordance. 


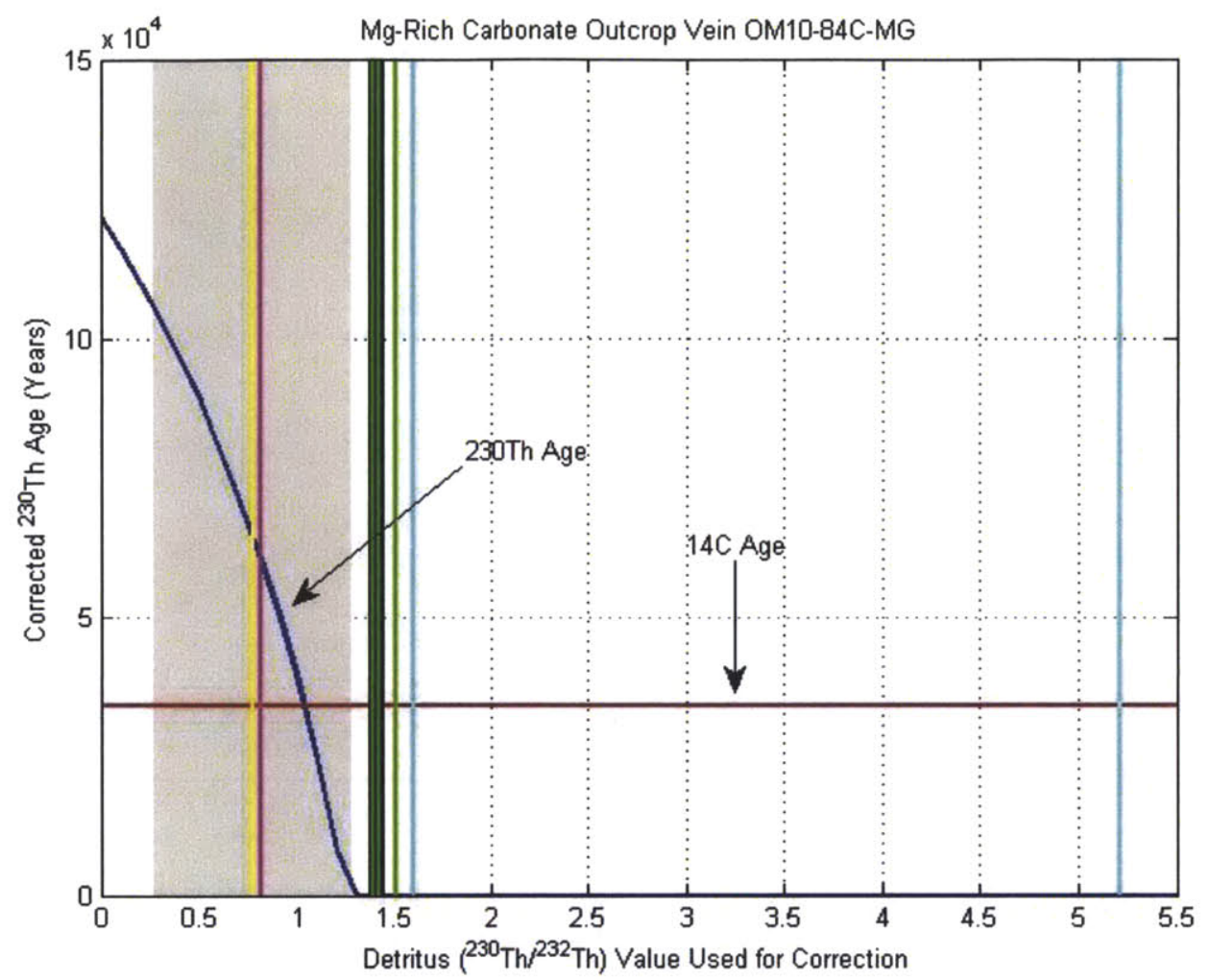

Figure B12: Sensitivity analysis figure showing the effect of the selected $\left({ }^{230} \mathrm{Th} /{ }^{232} \mathrm{Th}\right)$ correction value on the corrected ${ }^{230} \mathrm{Th}$ age calculated using Equation 1 in Chapter 3 . This figure is for Sample OM10-53C-MG, an Mg-rich carbonate outcroup vein from AlWuqbah Roadcut. The dark blue line indicates the calculated ${ }^{230} \mathrm{Th}$ age. Note that the uncorrected age plots at $\mathrm{x}=0$. For comparison, the ${ }^{14} \mathrm{C}$ age is plotted as the red line. The gray box indicates the $2 \sigma$ range of $\left({ }^{230} \mathrm{Th} /{ }^{232} \mathrm{Th}\right.$ ) for aluminosilicate detritus (literature compilation shown in Chapter 3, Figure 9, $\mathrm{n}=300$ ). Vertical lines indicate possible values to use for the initial $\left({ }^{230} \mathrm{Th} /{ }^{232} \mathrm{Th}\right)$ : average of aluminosilicate detritus compilation (yellow line), equilibrium Bulk Silicate Earth (pink line), highly-altered peridotites (green lines; note that a fourth peridotite plots off the figure at 12.367), wadi sediments (turquoise lines), and recently-formed travertine precipitates (black lines). The choice of the initial $\left({ }^{230} \mathrm{Th} /{ }^{232} \mathrm{Th}\right)$ has a very significant impact on the determined ${ }^{230} \mathrm{Th}$ age, which ranges from $>100,000$ years to zero depending on the correction value selected. A $\left({ }^{230} \mathrm{Th} /{ }^{232} \mathrm{Th}\right)$ correction value of approximately 1 brings the corrected ${ }^{230} \mathrm{Th}$ age into concordance with the ${ }^{14} \mathrm{C}$ age. 


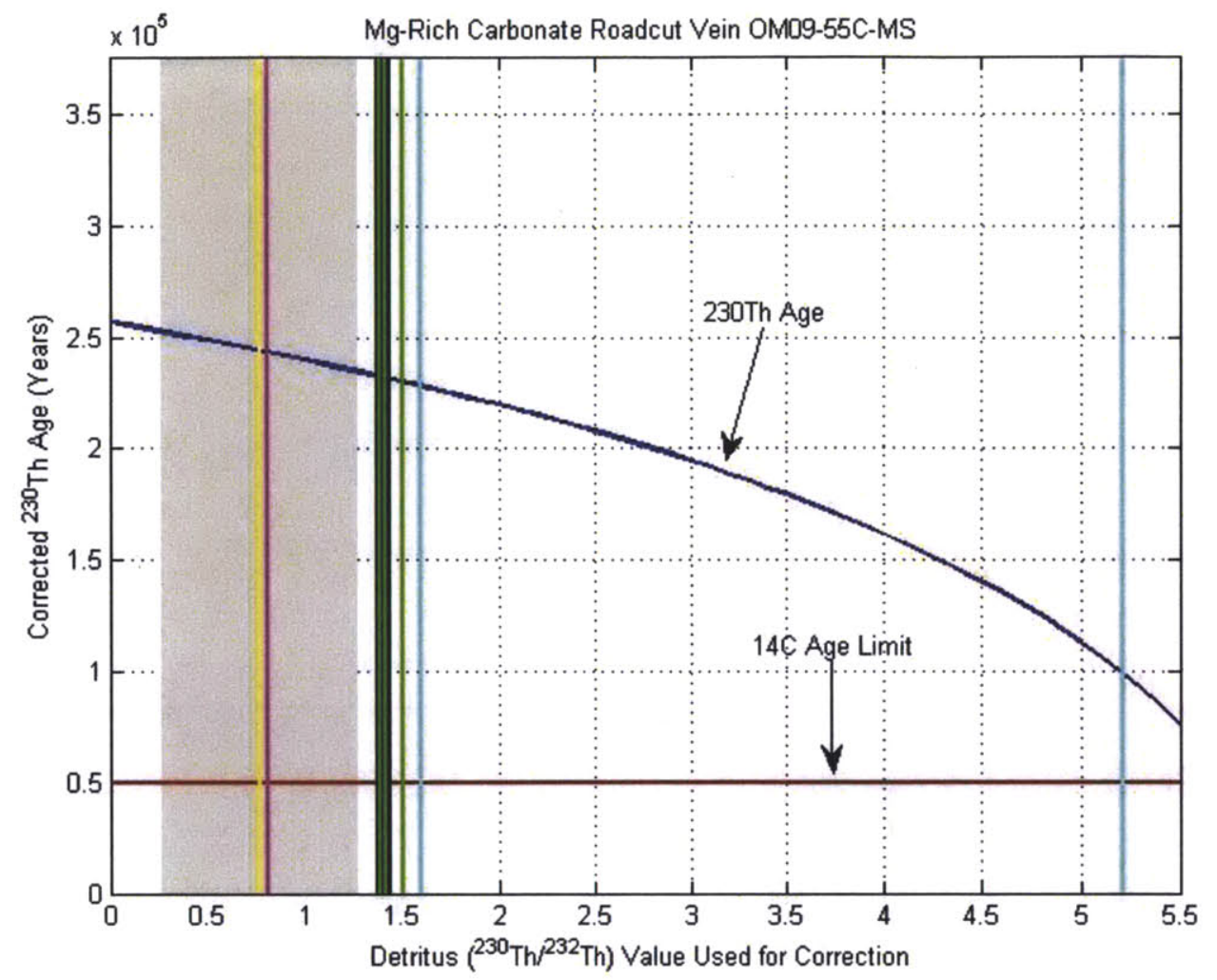

Figure B13: Sensitivity analysis figure showing the effect of the selected $\left({ }^{230} \mathrm{Th} /{ }^{232} \mathrm{Th}\right)$ correction value on the corrected ${ }^{230} \mathrm{Th}$ age calculated using Equation 1. This figure is for sample OM09-55C-MS, an Mg-rich carbonate roadcut vein from Fanja Roadcut. The dark blue line indicates the corrected ${ }^{230} \mathrm{Th}$ age. Note that the uncorrected ${ }^{230} \mathrm{Th}$ age plots at $\mathrm{x}=0$. For comparison, the ${ }^{14} \mathrm{C}$ age is plotted as the red line. The gray box indicates the $2 \sigma$ range of $\left({ }^{230} \mathrm{Th} /{ }^{232} \mathrm{Th}\right)$ for aluminosilicate detritus (literature compilation shown in Figure $9, \mathrm{n}=300)$. Vertical lines indicate possible values to use for the $\left({ }^{230} \mathrm{Th} /{ }^{232} \mathrm{Th}\right)$ correction value: average of aluminosilicate detritus compilation (yellow line), equilibrium Bulk Silicate Earth (pink line), highly-altered peridotites (green lines; note that a fourth peridotite plots off the figure at 12.367), wadi sediments (turquoise lines), and recently-formed travertine precipitates (black lines). The choice of the $\left({ }^{230} \mathrm{Th} /{ }^{232} \mathrm{Th}\right.$ ) correction value has a significant impact on the determined ${ }^{230} \mathrm{Th}$ age, which ranges from $\sim 250,000$ years to $\sim 60,000$ years. This is consistent with the ${ }^{14} \mathrm{C}$ dead $(>50,000$ yr BP) age of this sample. 


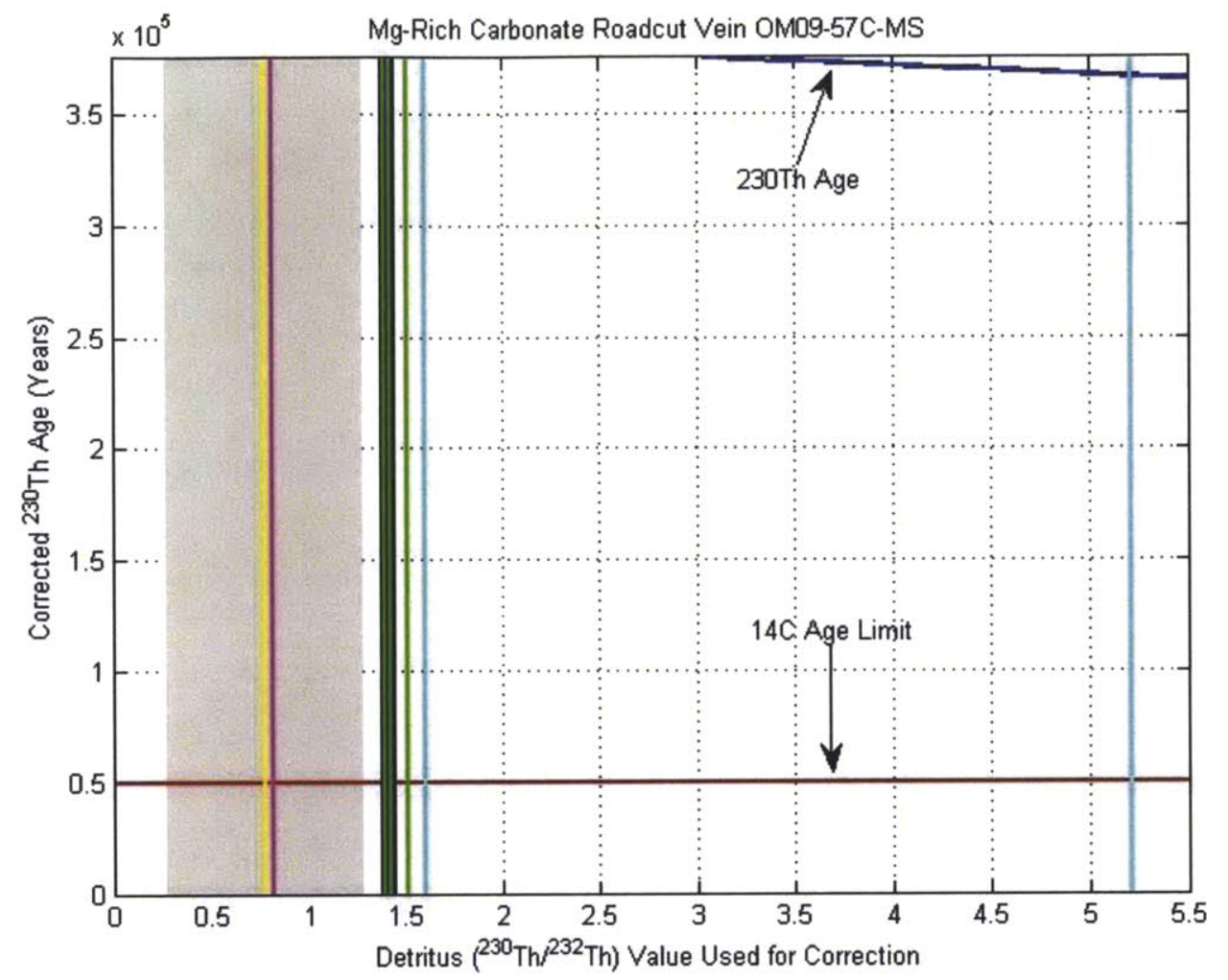

Figure B14: Sensitivity analysis figure showing the effect of the selected $\left({ }^{230} \mathrm{Th} /{ }^{232} \mathrm{Th}\right)$ correction value on the corrected ${ }^{230} \mathrm{Th}$ age calculated using Equation 1. This figure is for sample OM09-57C-MS, an Mg-rich carbonate roadcut vein from Fanja Roadcut. The dark blue line indicates the corrected ${ }^{230} \mathrm{Th}$ age. Note that the uncorrected ${ }^{230} \mathrm{Th}$ age plots at $\mathrm{x}=0$. For comparison, the ${ }^{14} \mathrm{C}$ age is plotted as the red line. The gray box indicates the $2 \sigma$ range of $\left({ }^{230} \mathrm{Th} /{ }^{232} \mathrm{Th}\right)$ for aluminosilicate detritus (literature compilation shown in Figure $9, \mathrm{n}=300)$. Vertical lines indicate possible values to use for the $\left({ }^{230} \mathrm{Th} /{ }^{232} \mathrm{Th}\right)$ correction value: average of aluminosilicate detritus compilation (yellow line), equilibrium Bulk Silicate Earth (pink line), highly-altered peridotites (green lines; note that a fourth peridotite plots off the figure at 12.367), wadi sediments (turquoise lines), and recently-formed travertine precipitates (black lines). The choice of the $\left({ }^{230} \mathrm{Th} /{ }^{232} \mathrm{Th}\right.$ ) correction value has minimal impact on the determined ${ }^{230} \mathrm{Th}$ age, which remains greater than 350,000 years. This is consistent with the ${ }^{14} \mathrm{C}$ dead $(>50,000 \mathrm{yr} \mathrm{BP})$ age of this sample. 


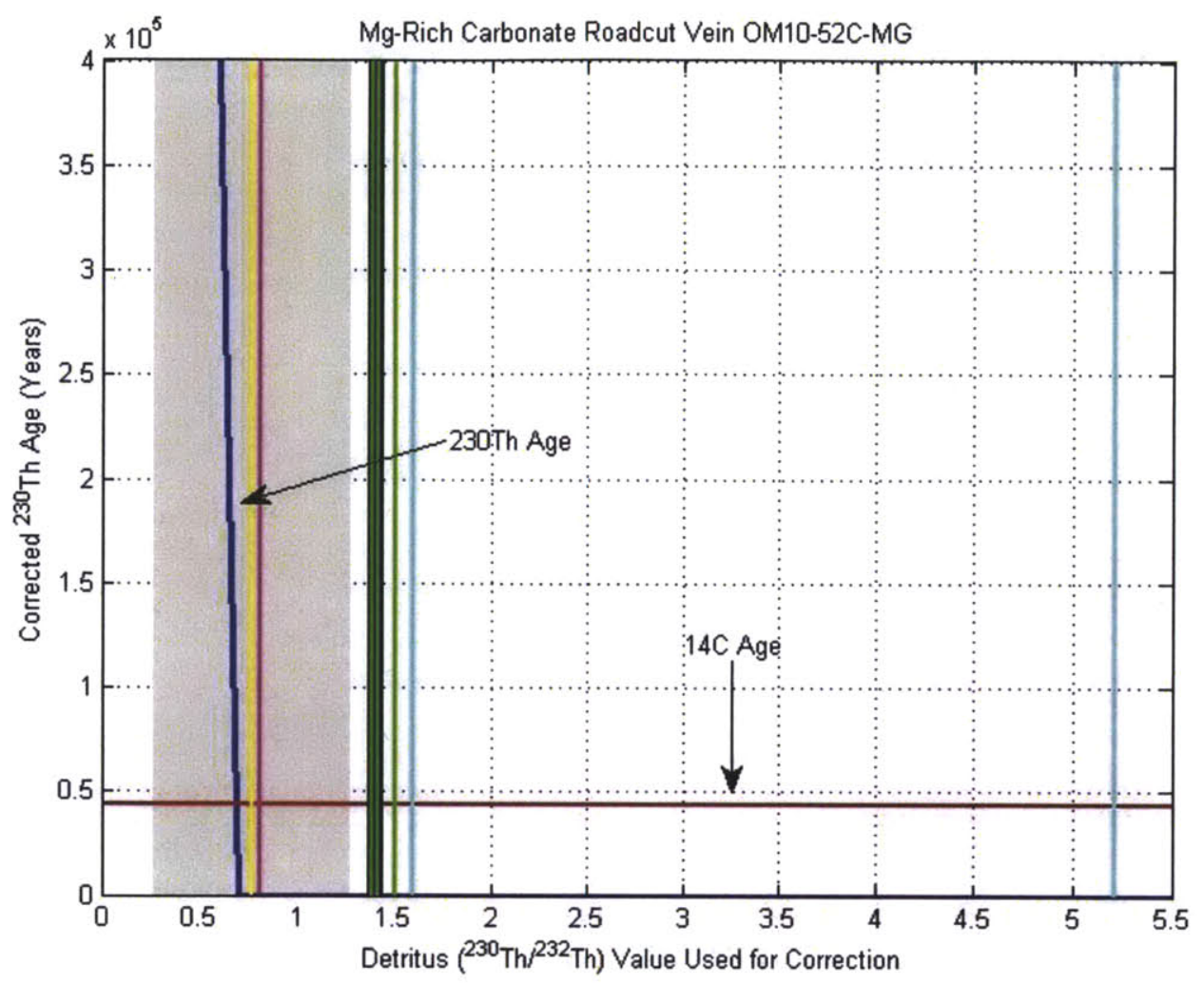

Figure B15: Sensitivity analysis figure showing the effect of the $\left({ }^{230} \mathrm{Th} /{ }^{232} \mathrm{Th}\right)$ correction value on the ${ }^{230}$ Th-corrected age calculated using Equation 1 in Chapter 3. This figure is for sample OM10-52C-MG, an Mg-rich carbonate roadcut vein from Al-Wuqbah Roadcut. The dark blue line indicates the calculated ${ }^{230} \mathrm{Th}$ age. Note that the uncorrected age plots at $\mathrm{x}=0$. For comparison, the ${ }^{14} \mathrm{C}$ age is plotted as the red line. The gray box indicates the $2 \sigma$ range of $\left({ }^{230} \mathrm{Th} /{ }^{232} \mathrm{Th}\right.$ ) for aluminosilicate detritus (literature compilation shown in Chapter 3, Figure 9, $\mathrm{n}=300$ ). Vertical lines indicate possible values to use for the initial $\left({ }^{230} \mathrm{Th} /{ }^{232} \mathrm{Th}\right)$ : average of aluminosilicate detritus compilation (yellow line), equilibrium Bulk Silicate Earth (pink line), highly-altered peridotites (green lines; note that a fourth peridotite plots off the figure at 12.367), wadi sediments (turquoise lines), and recently-formed travertine precipitates (black lines). Due to the high $\mathrm{Th} / \mathrm{U}$ ratio of this sample, no realistic uncorrected or corrected ${ }^{230} \mathrm{Th}$ ages can be calculated for this sample. 